Cochrane Database of Systematic Reviews

\title{
Chlorhexidine mouthrinse as an adjunctive treatment for gingival health (Review)
}

James P, Worthington HV, Parnell C, Harding M, Lamont T, Cheung A, Whelton H, Riley P

James P, Worthington HV, Parnell C, Harding M, Lamont T, Cheung A, Whelton H, Riley P.

Chlorhexidine mouthrinse as an adjunctive treatment for gingival health.

Cochrane Database of Systematic Reviews 2017, Issue 3. Art. No.: CD008676.

DOI: 10.1002/14651858.CD008676.pub2.

www.cochranelibrary.com 
TABLE OF CONTENTS

ABSTRACT 1

PLAIN LANGUAGE SUMMARY

SUMMARY OF FINDINGS

BACKGROUND

OBJECTIVES

METHODS

Figure 1.

RESULTS

Figure 2.

DISCUSSION

AUTHORS' CONCLUSIONS

ACKNOWLEDGEMENTS

REFERENCES

CHARACTERISTICS OF STUDIES .....

DATA AND ANALYSES

Analysis 1.1. Comparison 1: CHX versus placebo/control mouthrinse or no mouthrinse, Outcome 1: Gingival Index (0-3) 4-6 weeks

Analysis 1.2. Comparison 1: $\mathrm{CHX}$ versus placebo/control mouthrinse or no mouthrinse, Outcome 2: Gingival Index (0-3) 6 months

Analysis 1.3. Comparison 1: $\mathrm{CHX}$ versus placebo/control mouthrinse or no mouthrinse, Outcome 3: Gingival bleeding 4-6 weeks

Analysis 1.4. Comparison 1: CHX versus placebo/control mouthrinse or no mouthrinse, Outcome 4: Gingival bleeding 6 months

Analysis 1.5. Comparison 1: $\mathrm{CHX}$ versus placebo/control mouthrinse or no mouthrinse, Outcome 5: Plaque 4-6 weeks ............ Analysis 1.6. Comparison 1: CHX versus placebo/control mouthrinse or no mouthrinse, Outcome 6: Plaque 4-6 weeks PI (0-3) .. Analysis 1.7. Comparison 1: CHX versus placebo/control mouthrinse or no mouthrinse, Outcome 7: Plaque 4-6 weeks TQH (0-5)

Analysis 1.8. Comparison 1: CHX versus placebo/control mouthrinse or no mouthrinse, Outcome 8: Plaque 6 months ............ Analysis 1.9. Comparison 1: CHX versus placebo/control mouthrinse or no mouthrinse, Outcome 9: Plaque 6 months PI (0-3) .. Analysis 1.10. Comparison 1: $\mathrm{CHX}$ versus placebo/control mouthrinse or no mouthrinse, Outcome 10: Plaque 6 months TQH $(0-5)$

Analysis 1.11. Comparison 1: CHX versus placebo/control mouthrinse or no mouthrinse, Outcome 11: Calculus 4-6 weeks .....

Analysis 1.12. Comparison 1: $\mathrm{CHX}$ versus placebo/control mouthrinse or no mouthrinse, Outcome 12: Calculus 7-12 weeks .... Analysis 1.13. Comparison 1: $\mathrm{CHX}$ versus placebo/control mouthrinse or no mouthrinse, Outcome 13: Calculus 6 months ....... Analysis 1.14. Comparison 1: $\mathrm{CHX}$ versus placebo/control mouthrinse or no mouthrinse, Outcome 14: Tooth staining 4-6 weeks dichotomous

Analysis 1.15. Comparison 1: CHX versus placebo/control mouthrinse or no mouthrinse, Outcome 15: Tooth staining 7-12 weeks dichotomous

Analysis 1.16. Comparison 1: CHX versus placebo/control mouthrinse or no mouthrinse, Outcome 16: Tooth staining 4-6 weeks

Analysis 1.17. Comparison 1: $\mathrm{CHX}$ versus placebo/control mouthrinse or no mouthrinse, Outcome 17: Tooth staining 7-12 weeks

Analysis 1.18. Comparison 1: CHX versus placebo/control mouthrinse or no mouthrinse, Outcome 18: Tooth staining 6 months ADDITIONAL TABLES

APPENDICES

WHAT'S NEW

HISTORY

CONTRIBUTIONS OF AUTHORS

DECLARATIONS OF INTEREST

SOURCES OF SUPPORT

DIFFERENCES BETWEEN PROTOCOL AND REVIEW

NOTES

INDEX TERMS 
[Intervention Review]

\section{Chlorhexidine mouthrinse as an adjunctive treatment for gingival health}

Patrice James ${ }^{1}$, Helen V Worthington ${ }^{2}$, Carmel Parnell 3 , Mairead Harding 4 , Thomas Lamont ${ }^{5}$, Andrea Cheung6, Helen Whelton 7 , Philip Riley ${ }^{2}$

1Oral Health Services Research Centre, Cork University Dental School and Hospital, Wilton, Cork, Ireland. ${ }^{2}$ Cochrane Oral Health, Division of Dentistry, School of Medical Sciences, Faculty of Biology, Medicine and Health, The University of Manchester, Manchester, UK. ${ }^{3} \mathrm{HSE}$ Louth Meath Dental Service, Navan, Ireland. ${ }^{4}$ Oral Health Services Research Centre, Cork University Dental School and Hospital (UCC), Wilton, Cork, and HSE South (CHO 4), Cork, Ireland. ${ }^{5}$ School of Dentistry, University of Dundee, Dundee, UK. ${ }^{6}$ Cork University Dental School and Hospital (UCC), Wilton, Cork, Ireland. ${ }^{7}$ School of Dentistry, University of Leeds, Leeds, UK

Contact: Patrice James, p.james@ucc.ie.

Editorial group: Cochrane Oral Health Group.

Publication status and date: Stable (no update expected for reasons given in 'What's new'), published in Issue 12, 2021.

Citation: James P, Worthington HV, Parnell C, Harding M, Lamont T, Cheung A, Whelton H, Riley P. Chlorhexidine mouthrinse as an adjunctive treatment for gingival health. Cochrane Database of Systematic Reviews 2017, Issue 3. Art. No.: CD008676. DOI: 10.1002/14651858.CD008676.pub2.

Copyright ( 2017 The Cochrane Collaboration. Published by John Wiley \& Sons, Ltd.

\section{A B S T R A C T}

\section{Background}

Dental plaque associated gingivitis is a reversible inflammatory condition caused by accumulation and persistence of microbial biofilms (dental plaque) on the teeth. It is characterised by redness and swelling of the gingivae (gums) and a tendency for the gingivae to bleed easily. In susceptible individuals, gingivitis may lead to periodontitis and loss of the soft tissue and bony support for the tooth. It is thought that chlorhexidine mouthrinse may reduce the build-up of plaque thereby reducing gingivitis.

\section{Objectives}

To assess the effectiveness of chlorhexidine mouthrinse used as an adjunct to mechanical oral hygiene procedures for the control of gingivitis and plaque compared to mechanical oral hygiene procedures alone or mechanical oral hygiene procedures plus placebo/control mouthrinse. Mechanical oral hygiene procedures were toothbrushing with/without the use of dental floss or interdental cleaning aids and could include professional tooth cleaning/periodontal treatment.

To determine whether the effect of chlorhexidine mouthrinse is influenced by chlorhexidine concentration, or frequency of rinsing (once/ day versus twice/day).

To report and describe any adverse effects associated with chlorhexidine mouthrinse use from included trials.

\section{Search methods}

Cochrane Oral Health's Information Specialist searched the following databases: Cochrane Oral Health's Trials Register (to 28 September 2016); the Cochrane Central Register of Controlled Trials (CENTRAL; 2016, Issue 8) in the Cochrane Library (searched 28 September 2016); MEDLINE Ovid (1946 to 28 September 2016); Embase Ovid (1980 to 28 September 2016); and CINAHL EBSCO (Cumulative Index to Nursing and Allied Health Literature; 1937 to 28 September 2016). We searched ClinicalTrials.gov and the World Health Organization International Clinical Trials Registry Platform for ongoing trials. No restrictions were placed on the language or date of publication when searching the electronic databases.

\section{Selection criteria}

We included randomised controlled trials assessing the effects of chlorhexidine mouthrinse used as an adjunct to mechanical oral hygiene procedures for at least 4 weeks on gingivitis in children and adults. Mechanical oral hygiene procedures were toothbrushing with/without 
use of dental floss or interdental cleaning aids and could include professional tooth cleaning/periodontal treatment. We included trials where participants had gingivitis or periodontitis, where participants were healthy and where some or all participants had medical conditions or special care needs.

\section{Data collection and analysis}

Two review authors independently screened the search results extracted data and assessed the risk of bias of the included studies. We attempted to contact study authors for missing data or clarification where feasible. For continuous outcomes, we used means and standard deviations to obtain the mean difference (MD) and 95\% confidence interval (CI). We combined MDs where studies used the same scale and standardised mean differences (SMDs) where studies used different scales. For dichotomous outcomes, we reported risk ratios (RR) and $95 \%$ Cls. Due to anticipated heterogeneity we used random-effects models for all meta-analyses.

\section{Main results}

We included 51 studies that analysed a total of 5345 participants. One study was assessed as being at unclear risk of bias, with the remaining 50 being at high risk of bias, however, this did not affect the quality assessments for gingivitis and plaque as we believe that further research is very unlikely to change our confidence in the estimate of effect.

\section{Gingivitis}

After 4 to 6 weeks of use, chlorhexidine mouthrinse reduced gingivitis (Gingival Index (GI) 0 to 3 scale) by 0.21 ( $95 \%$ Cl 0.11 to 0.31 ) compared to placebo, control or no mouthrinse (10 trials, 805 participants with mild gingival inflammation (mean score 1 on the GI scale) analysed, high-quality evidence). A similar effect size was found for reducing gingivitis at 6 months. There were insufficient data to determine the reduction in gingivitis associated with chlorhexidine mouthrinse use in individuals with mean GI scores of 1.1 to 3 (moderate or severe levels of gingival inflammation).

\section{Plaque}

Plaque was measured by different indices and the SMD at 4 to 6 weeks was 1.45 (95\% $\mathrm{Cl} 1.00$ to 1.90$)$ standard deviations lower in the chlorhexidine group (12 trials, 950 participants analysed, high-quality evidence), indicating a large reduction in plaque. A similar large reduction was found for chlorhexidine mouthrinse use at 6 months.

\section{Extrinsic tooth staining}

There was a large increase in extrinsic tooth staining in participants using chlorhexidine mouthrinse at 4 to 6 weeks. The SMD was 1.07 (95\% $\mathrm{Cl} 0.80$ to 1.34 ) standard deviations higher (eight trials, 415 participants analysed, moderate-quality evidence) in the chlorhexidine mouthrinse group. There was also a large increase in extrinsic tooth staining in participants using chlorhexidine mouthrinse at 7 to 12 weeks and 6 months.

\section{Calculus}

Results for the effect of chlorhexidine mouthrinse on calculus formation were inconclusive.

\section{Effect of concentration and frequency of rinsing}

There were insufficient data to determine whether there was a difference in effect for either chlorhexidine concentration or frequency of rinsing.

\section{Other adverse effects}

The adverse effects most commonly reported in the included studies were taste disturbance/alteration (reported in 11 studies), effects on the oral mucosa including soreness, irritation, mild desquamation and mucosal ulceration/erosions (reported in 13 studies) and a general burning sensation or a burning tongue or both (reported in nine studies).

\section{Authors' conclusions}

There is high-quality evidence from studies that reported the Löe and Silness Gingival Index of a reduction in gingivitis in individuals with mild gingival inflammation on average (mean score of 1 on the 0 to $3 \mathrm{Gl}$ scale) that was not considered to be clinically relevant. There is high-quality evidence of a large reduction in dental plaque with chlorhexidine mouthrinse used as an adjunct to mechanical oral hygiene procedures for 4 to 6 weeks and 6 months. There is no evidence that one concentration of chlorhexidine rinse is more effective than another. There is insufficient evidence to determine the reduction in gingivitis associated with chlorhexidine mouthrinse use in individuals with mean GI scores of 1.1 to 3 indicating moderate or severe levels of gingival inflammation. Rinsing with chlorhexidine mouthrinse for 4 weeks or longer causes extrinsic tooth staining. In addition, other adverse effects such as calculus build up, transient taste disturbance and effects on the oral mucosa were reported in the included studies. 


\section{PLAIN LANGUAGE SUMMARY}

\section{Chlorhexidine mouthrinse to reduce gingivitis and plaque build-up}

\section{Review question}

Does the use of chlorhexidine mouthrinse (a broad spectrum antiseptic) in addition to other conventional tooth cleaning help to control and improve gingivitis (inflammation of the gums)? Does the frequency of rinsing or the concentration of the solution affect the result and are there any undesirable side effects?

\section{Background}

Gingivitis is a reversible condition when gums become red, swollen and can bleed easily. Gingivitis is also very common - studies suggest that as many as $50 \%$ to $90 \%$ of adults in the UK and USA suffer from it. In susceptible people gingivitis may lead to periodontitis, which is not reversible. In periodontitis inflammation is accompanied by loss of ligaments and bone supporting the teeth. If untreated it may eventually lead to tooth loss. Severe periodontitis is the sixth most widespread disease globally.

It is recognised that maintaining a high standard of oral hygiene is important for the prevention and treatment of gingivitis. Toothbrushing is the main method for maintaining good oral hygiene. Other cleaning methods commonly used include dental floss, interdental brushes and scaling and polishing carried out by a dental professional. Some people have difficulty controlling plaque build-up and preventing gingivitis using only conventional tooth cleaning. Therefore people sometimes use mouthrinses containing chlorhexidine in addition to conventional tooth cleaning. These mouthrinses are readily available over the counter; prescriptions generally not being required outside the USA.

\section{Study characteristics}

We included 51 studies that analysed a total of 5345 participants. The evidence in this review is up to date as of 28 September 2016 . Generally study participants were children and adults who had gingivitis or periodontitis, were able to use usual tooth cleaning methods and were healthy. We did not exclude studies where some or all participants had medical conditions or special care needs as we considered the use of mouthrinses with chlorhexidine to be particularly relevant to them. The included studies assessed the effects of chlorhexidine mouthrinse used for at least 4 weeks in addition to conventional tooth cleaning on gingivitis in children and adults.

\section{Key results}

There is high-quality evidence that the use of mouthrinses containing chlorhexidine in addition to usual toothbrushing and cleaning for 4 to 6 weeks or 6 months leads to a large reduction in the build-up of plaque. There is also high-quality evidence of a moderate reduction in gingivitis in people with a mild level of it, although because the level of disease was already low this is not considered clinically important. The nature of the available evidence does not allow us to determine the level of reduction of gingivitis in people with moderate to severe levels of it.

There was no evidence that one concentration or strength of chlorhexidine rinse was more effective than another.

Rinsing for 4 weeks or longer causes tooth staining, which requires scaling and polishing carried out by a dental professional. Other side effects have been reported, including build-up of calculus (tartar), temporary taste disturbance and temporary shedding of/damage to the lining of the mouth.

\section{Quality of the evidence}

One study was assessed as being at unclear risk of bias, with the remaining 50 being at high risk of bias, however this did not affect the quality assessments for gingivitis and plaque as we believe that further research is very unlikely to change our confidence in the estimate of effect. 
SUMMARY OF FINDINGS

Summary of findings 1 . Summary of findings

Chlorhexidine mouthrinse compared with placebo/control mouthrinse/no mouthrinse for gingival health

Patient or population: adults and children with gingivitis

\section{Settings: any}

Intervention: chlorhexidine mouthrinse

Comparison: placebo/control mouthrinse or no mouthrinse

\begin{tabular}{|c|c|c|c|c|c|c|}
\hline \multirow[t]{3}{*}{ Outcomes } & \multicolumn{2}{|c|}{ Illustrative comparative risks* $(95 \% \mathrm{Cl})$} & \multirow{3}{*}{$\begin{array}{l}\text { Relative effect } \\
(95 \% \mathrm{Cl})\end{array}$} & \multirow{3}{*}{$\begin{array}{l}\text { Number of par- } \\
\text { ticipants } \\
\text { (studies) }\end{array}$} & \multirow{3}{*}{$\begin{array}{l}\text { Quality of the } \\
\text { evidence } \\
\text { (GRADE) }\end{array}$} & \multirow[t]{3}{*}{ Comments } \\
\hline & Assumed risk & Corresponding risk & & & & \\
\hline & Control & Chlorhexidine & & & & \\
\hline $\begin{array}{l}\text { Gingival Index } 4 \\
\text { to } 6 \text { weeks (Löe } \\
\text { and Silness Gingi- } \\
\text { val Index) (0 to } 3 \\
\text { on an increasing } \\
\text { scale) }\end{array}$ & $\begin{array}{l}\text { The mean gingivi- } \\
\text { tis scores ranged } \\
\text { across control } \\
\text { groups from } 0.17 \\
\text { to } 1.40^{1}\end{array}$ & $\begin{array}{l}\text { The mean gingivi- } \\
\text { tis score in the } \\
\text { chlorhexidine group } \\
\text { was } 0.21 \text { lower ( } 0.11 \\
\text { to } 0.31 \text { lower) }\end{array}$ & & $\begin{array}{l}805 \\
\text { (10 trials) }\end{array}$ & $\begin{array}{l}\oplus \oplus \oplus \oplus^{2,3} \\
\text { high }\end{array}$ & $\begin{array}{l}\text { The effect size at } 6 \text { months was similar } \\
\text { Insufficient evidence for differences in effect } \\
\text { size for different chlorhexidine concentration } \\
\text { or frequency of use } \\
\text { Insufficient evidence to determine the effect } \\
\text { size in individuals with moderate or severe lev- } \\
\text { els of gingival inflammation on average (mean } \\
\text { GI scores } 1.1 \text { to 3) }\end{array}$ \\
\hline $\begin{array}{l}\text { Plaque } 4 \text { to } 6 \\
\text { weeks } \\
\text { (various increas- } \\
\text { ing scales includ- } \\
\text { ing Plaque In- } \\
\text { dex (0 to } 3 \text { scale) } \\
\text { and Turesky Mod- } \\
\text { ification of the } \\
\text { Quigley and Hein } \\
\text { Index (0 to } 5 \\
\text { scale)) }\end{array}$ & $\begin{array}{l}\text { Plaque Index } \\
\text { ranged from } 0.75 \\
\text { to } 1.06 \\
\text { Turesky Modi- } \\
\text { fication of the } \\
\text { Quigley and Hein } \\
\text { Index ranged } \\
\text { from } 1.2 \text { to } 3.3\end{array}$ & $\begin{array}{l}\text { The SMD was } \\
1.45 \text { lower in the } \\
\text { chlorhexidine group } \\
\text { indicating a large re- } \\
\text { duction in plaque } \\
\text { from } 1.00 \text { to } 1.90 \\
\text { standard deviations }\end{array}$ & & $\begin{array}{l}950 \\
\text { (12 trials) }\end{array}$ & $\begin{array}{l}\oplus \oplus \oplus \oplus^{2,3} \\
\text { high }\end{array}$ & $\begin{array}{l}\text { The effect for the Plaque Index ( } 4 \text { trials; } 223 \\
\text { participants) was } 0.58 \text { ( } 95 \% \mathrm{Cl} 0.39 \text { to } 0.78 \text { ) low- } \\
\text { er } \\
\text { The effect for the Turesky Modification of the } \\
\text { Quigley and Hein Index ( } 5 \text { trials; } 546 \text { partici- } \\
\text { pants) was } 0.78 \text { ( } 95 \% \mathrm{Cl} 0.70 \text { to } 0.85 \text { ) lower } \\
\text { There were also large effects for the plaque at } 6 \\
\text { months }\end{array}$ \\
\hline
\end{tabular}




\section{Tooth staining 4}

to 6 weeks

(various increas-

ing scales

\begin{tabular}{|c|c|}
\hline $\begin{array}{l}\text { The mean tooth } \\
\text { staining score } \\
\text { was measured on } \\
\text { different scales }\end{array}$ & $\begin{array}{l}\text { The SMD for tooth } \\
\text { staining in the } \\
\text { chlorhexidine group } \\
\text { was } \\
1.07 \text { ( } 0.80 \text { to } 1.34) \\
\text { standard deviations } \\
\text { higher }\end{array}$ \\
\hline
\end{tabular}

415

(8 trials) $\oplus \oplus \oplus \ominus^{4}$

moderate

Data have not been converted to original scale as many different scales are used. The SMD effect size is considered large

There were also 2 trials presenting dichotomous data showing large significant effect RR $5.41(95 \% \mathrm{Cl} 2.03$ to 14.47$)$

There was also a large effect for tooth staining for chlorhexidine at 7 to 12 weeks and 6 months

\section{Other adverse}

effects

22 trials reported at least 1 adverse effect apart from extrinsic tooth staining and calculus formation in the chlorhexidine rinse arms. The adverse effects most commonly reported were taste disturbance/alteration (reported in 11 trials), effects on the oral mucosa including mucosal irritation, soreness, mild desquamation, mucosal ulceration/erosions, oral mucosal lesions (reported in 13 trials) and a general burning sensation and/or a burning tongue (reported in 9 trials)

*The basis for the assumed risk (e.g. the median control group risk across trials) is provided in footnotes. The corresponding risk (and its $95 \% \mathrm{Cl}$ ) is based on the assumed risk in the comparison group and the relative effect of the intervention (and its $95 \% \mathrm{Cl}$ )

Cl: confidence interval; GI: Gingival Index; RR: risk ratio; SMD: standardised mean difference

GRADE Working Group grades of evidence

High quality: further research is very unlikely to change our confidence in the estimate of effect

Moderate quality: further research is likely to have an important impact on our confidence in the estimate of effect and may change the estimate

Low quality: further research is very likely to have an important impact on our confidence in the estimate of effect and is likely to change the estimate

Very low quality: we are very uncertain about the estimate

1 The mean gingivitis score for the control group was 0.93 (median is 1.0).

2Although most trials included in the meta-analyses were assessed as at high risk of bias we did not downgrade the GRADE assessments for this reason because we believe that further research is very unlikely to change our confidence in the estimate of effect.

${ }^{3}$ Not downgraded for high heterogeneity as results consistent.

${ }^{4}$ Downgraded as 8 trials at high risk of bias. 


\section{B A C K G R O U N D}

\section{Description of the condition}

Plaque-associated periodontal disease involves the inflammation of the gingivae (gums) and the supporting structures of the teeth. The first stage of the disease is plaque-associated gingivitis (inflammation of the gingivae), which is a reversible inflammatory condition characterised by redness and swelling of the gingivae and a tendency for the gingivae to bleed easily. It is caused by the accumulation and persistence of microbial biofilms (dental plaque) on the teeth (Löe 1965). Gingivitis is very common with studies suggesting that as many as $50 \%$ to $90 \%$ of adults in the UK and USA suffer from gingivitis (NICE 2012). In susceptible individuals, gingivitis may lead to the second stage of periodontal disease, periodontitis. Periodontitis is an irreversible condition, where inflammation of the gingivae is accompanied by connective tissue destruction and loss of the periodontal ligament and alveolar bone supporting the tooth. If untreated, periodontitis may ultimately lead to tooth loss. Severe periodontitis is the sixth most prevalent disease of man with a global age-standardised prevalence of $11.2 \%$ (Kassebaum 2014). It has a negative impact on oral-health-related quality of life (Al-Harthi 2013; Marcenes 2013; Needleman 2004; Tonetti 2015), affecting both physical function and social interaction (Cunha-Cruz 2007; Ng 2006). The response of the individual to gingival irritation by the microbial biofilm is modulated by local, systemic and genetic factors, and it is recognised that gingivitis will only progress to periodontitis in some individuals and at some sites. However as the microbial biofilm is a prerequisite for both conditions, gingival inflammation is considered a key risk factor in the initiation of periodontitis (Chapple 2015; Lang 2009). Indeed, consistent inflammation of the gingivae and gingival bleeding are predictive of future attachment loss and tooth loss (Schatzle 2004).

Although it is not possible to identify those individuals who will go on to develop severe periodontitis, the importance of maintaining a high standard of oral hygiene in the prevention and treatment of periodontal diseases is well recognised (Axelsson 2004; Ohrn 2009; van der Weijden 2011). Furthermore, the most important predictor of periodontal diseases, regardless of age is poor oral hygiene (Abdellatif 1987). Thus, the population approach to prevention of periodontal diseases should promote adequate plaque control and removal of the microbial biofilm for all individuals.

\section{Description of the intervention}

Mechanical disruption and removal of the microbial biofilm is commonly achieved by toothbrushing and interdental cleaning (Berchier 2008). However, adequate removal of the microbial biofilm with mechanical methods alone can be difficult for some, with many individuals finding it difficult to maintain an effective level of plaque control (Marsh 1992; Salzer 2015; Slot 2012; van der Weijden 2005; van der Weijden 2011; van der Weijden 2015). For these individuals, the level of plaque control that is achieved by regular mechanical cleaning alone may not be sufficient to prevent the onset or recurrence of periodontal diseases (Serrano 2015).Therefore chemical methods of removing the microbial biofilm such as antimicrobial mouthrinses have been recommended for use as either an adjunct to, or in some limited situations, a replacement for mechanical removal of the microbial biofilm.
Chlorhexidine is a broad spectrum bisbiguanide antiseptic which was first introduced into clinical medicine in 1953 in the form of an antiseptic cream (Eley 1999; Foulkes 1973). It is a strong base and is practically insoluble in water (Karpinski 2015). In clinical medicine its water soluble salts, in particular chlorhexidine digluconate, are most commonly used (Foulkes 1973; Karpinski 2015). In dentistry, chlorhexidine is used in a variety of formulations and vehicles, such as mouthrinses, gels, sprays and varnishes. Problems can be encountered when delivering chlorhexidine as a gel or a toothpaste as the chlorhexidine may bind to ingredients in the products reducing its activity (Eley 1999). Although considerable advances have been made in recent years in the formulation of these and other chlorhexidine vehicles, mouthrinses have the advantage of having a long history of use for oral health. They are already accepted as part of a normal oral hygiene regimen, used following normal mechanical tooth cleaning (van der Weijden 2015). Chlorhexidine-containing mouthrinses are marketed worldwide under a number of trade names and are in widespread use for chemical plaque control. This family of rinses are mainly indicated for use as adjuncts to mechanical cleaning, in specific clinical situations where mechanical oral hygiene is difficult, such as postsurgery, in individuals with intermaxillary fixation, in fixed appliance orthodontic therapy and in individuals with intellectual and physical disabilities (Addy 1986). Chlorhexidine mouthrinse is mainly available in concentrations of $0.1 \%, 0.12 \%$ or $0.2 \%$ chlorhexidine digluconate as well as in low concentration $(\leq 0.06 \%)$ rinse. The effect of chlorhexidine on the microbial biofilm is dosedependant (Keijser 2003). The optimum dose of chlorhexidine in a mouthrinse is considered to be $20 \mathrm{mg}$ twice daily (Löe 1970) equivalent to $10 \mathrm{~mL}$ of $0.2 \%$ chlorhexidine mouthrinse $(20 \mathrm{mg})$ or $15 \mathrm{~mL}$ of $0.12 \%$ chlorhexidine mouthrinse (18 mg) (Eley 1999; Keijser 2003). A rinse time of 30 seconds appears to be effective and acceptable although 60 -second rinse times are also advocated (Bonesvoll 1974; Keijser 2003).

\section{How the intervention might work}

The action of chlorhexidine mouthrinse as a treatment adjunct in managing inflammation of the gingivae involves its antimicrobial properties, which reduce the bacterial load of the microbial biofilm and should therefore reduce levels of inflammation and help prevent primary and secondary plaque-associated periodontal disease. Chlorhexidine digluconate has the ability to kill a wide variety of micro-organisms including gram-positive and gram-negative bacteria (aerobes and anaerobes) (Emilson 1977) as well as fungi including yeasts (Greenstein 1986; Puig Silla 2008). When chlorhexidine mouthrinse is used, the positively charged (cationic) chlorhexidine molecule binds to the negatively charged microbial cell wall and interferes with the osmotic equilibrium of the micro-organism. Depending on the dose, chlorhexidine can have a bacteriostatic or bacteriocidal effect. At low concentrations, chlorhexidine is bacteriostatic causing leakage of low molecular weight substances from the microbial cell and inhibiting reproduction. At higher concentrations chlorhexidine is bacteriocidal and causes cell death by precipitating the cytoplasmic contents of the microbial cell (Greenstein 1986; Puig Silla 2008). This ability of chlorhexidine to adsorb and adhere to negatively charged surfaces is central to its success as an antimicrobial agent. The chlorhexidine molecule has the ability to adsorb to anionic (negatively charged) substrates such as hydroxyapatite, pellicle, salivary glycoproteins and mucous membranes exerting an immediate bacteriocidal effect. When 
slowly released over time from the pellicle-coated enamel surface, it provides a prolonged bacteriostatic effect in vivo (Bonesvoll 1974; Bonesvoll 1974a; Bonesvoll 1978; Jenkins 1988; Rolla 1971). This property of chlorhexidine is known as its substantivity and is what sets chlorhexidine apart from many other antimicrobial agents.

Chlorhexidine mouthrinse use is associated with a number of local adverse effects the most common being the formation of brown staining on the teeth and oral tissues, particularly the tongue (Addy 1986). Several theories have been put forward to explain the cause of this characteristic staining, however most evidence points to the staining being a result of precipitation of anionic dietary chromogens (e.g. from tea, coffee, tannins from wine) onto adsorbed chlorhexidine cations (Addy 1985; Addy 1985a; Watts 2001). Other less common local adverse effects have also been reported including supragingival calculus accumulation (Eley 1999; Mandel 1994; Van Strydonck 2012), oral mucosal lesions (Addy 1986; Flotra 1971; Van Strydonck 2012), and altered taste perception (Addy 1986; Eley 1999; Marinone 2000; Van Strydonck 2012). The occurrence of side effects tends to be reduced with lower chlorhexidine concentrations (Addy 1986; Cumming and Löe 1973; Flotra 1971; Keijser 2003). With the exception of extrinsic staining of the teeth and calculus accumulation which require removal by professional tooth cleaning, the adverse effects are transient and resolve once chlorhexidine mouthrinse use has ceased (Flotra 1971; Greenstein 1986). These local adverse effects limit the use of chlorhexidine to short or moderate term use in specific clinical circumstances (van der Weijden 2015). Chlorhexidine is poorly absorbed by the oral tissues and the gastrointestinal tract and is considered to have very low toxicity (Foulkes 1973; Greenstein 1986). Parotid gland swelling has been reported following chlorhexidine mouthrinse use (Addy 1986; Eley 1999; van der Weijden 2010). There have been rare reports of type 1 hypersensitivity reactions to chlorhexidine used in the mouth or on the lips (Pemberton 2012) but severe reactions such as anaphylaxis do not appear to have occurred as a result of using chlorhexidine mouthrinse to maintain periodontal health.

\section{Why it is important to do this review}

Chlorhexidine mouthrinse is readily available and a prescription is generally not required outside the USA (Moran 2008). Its efficacy in controlling plaque and gingivitis in humans in the absence of active oral hygiene was first demonstrated over 40 years ago by Harald Löe and colleagues (Löe 1970). Nowadays chlorhexidine mouthrinse is mostly used as an adjunct to regular mechanical oral hygiene and is commonly regarded as the gold standard against which other antiplaque agents are measured (Jones 1997; Marsh 1992). The last decade has seen the publication of two meta-analyses (Gunsolley 2006; Gunsolley 2010) and a number of systematic reviews (including one metareview) of the effectiveness of chlorhexidine mouthrinse (Boyle 2014; Serrano 2015; van der Weijden 2015; Van Strydonck 2012). In most of these publications chlorhexidine mouthrinse was one of a number of antiplaque chemical agents under review. The one dedicated review of the effectiveness of chlorhexidine mouthrinse compared to placebo, control or regular oral hygiene employed slightly different inclusion criteria to this review but included tooth staining as an outcome in addition to gingivitis and plaque (Van Strydonck 2012). However, the search for this review was conducted in April 2011 and adverse effects other than extrinsic tooth staining were not prespecified outcomes in the review.
Given the numerous clinical indications for the use of chlorhexidine mouthrinse for gingivitis and plaque control, and the ready availability of chlorhexidine mouthrinse over-the-counter in many parts of the world, it is important to conduct a Cochrane systematic review of chlorhexidine mouthrinse as an adjunctive treatment for gingival health in order to provide, rigorous, up-to-date evidence for patients, oral health practitioners and policy makers. This should take into account the risk of bias of the studies that have been conducted, as well as assessing the possible adverse effects of the chlorhexidine mouthrinse.

\section{O B JECTIVES}

To assess the effectiveness of chlorhexidine mouthrinse used as an adjunct to mechanical oral hygiene procedures for the control of gingivitis and plaque compared to mechanical oral hygiene procedures alone or mechanical oral hygiene procedures plus placebo/control mouthrinse. Mechanical oral hygiene procedures were toothbrushing with/without the use of dental floss or interdental cleaning aids and could include professional tooth cleaning/periodontal treatment.

To determine whether the effect of chlorhexidine mouthrinse is influenced by chlorhexidine concentration, or frequency of rinsing (once/day versus twice/day).

To report and describe any adverse effects associated with chlorhexidine mouthrinse use (reporting of adverse effects is limited to those detailed in the included trials).

\section{METHODS}

\section{Criteria for considering studies for this review}

\section{Types of studies}

We included parallel-group randomised controlled trials (RCTs) irrespective of language or publication status where the duration of mouthrinsing was at least 4 weeks. The minimum duration of rinsing was selected to reflect the 'real life' use of chlorhexidine as a short-term adjunct to mechanical oral hygiene procedures. Studies where the duration of mouthrinsing was less than 4 weeks were excluded. Cross-over studies were excluded due to concerns that chlorhexidine could exert an effect beyond the washout period. It would not be possible to conduct a split-mouth study of mouthrinse use, however split-mouth studies comparing different scaling and root planing regimens and different periodontal surgical techniques often incorporate a chlorhexidine and placebo/control comparison. Such study designs were considered inappropriate to answer the question posed by this review and were excluded. We would have included properly designed cluster-RCTs if any such studies had met the inclusion criteria.

\section{Types of participants}

We included RCTs involving children or adults with gingivitis or periodontitis provided they were capable of performing normal mechanical oral hygiene procedures (in accordance with other Cochrane reviews, we classified all participants aged 16 years or less as children and those older than 16 years as adults). We included trials where some or all of the participants had medical conditions or special care needs as we considered the intervention to be particularly relevant to these individuals/groups. 


\section{Types of interventions}

Experimental intervention: chlorhexidine mouthrinse (used at any concentration, volume, frequency or duration of rinsing) used in conjunction with mechanical oral hygiene procedures (toothbrushing with/without the use of dental floss or interdental cleaning aids and could include professional tooth cleaning/ periodontal treatment).

Comparator interventions: mechanical oral hygiene alone (toothbrushing with/without the use of dental floss or interdental cleaning aids and could include professional tooth cleaning/ periodontal treatment), or mechanical oral hygiene used in conjunction with placebo/control mouthrinse.

We included studies:

- where the chlorhexidine mouthrinse also contained fluoride;

- where gum care or antigingivitis dentifrices (that did not contain chlorhexidine) were used for mechanical oral hygiene provided that they were used in both experimental and comparator arms;

- with and without baseline prophylaxis (scale and polish) but all study arms had to have the same treatment.

We excluded studies:

- where chlorhexidine mouthrinse was used as a monotherapy in the absence of mechanical oral hygiene procedures;

- where the chlorhexidine mouthrinse formed part of a combined intervention with other agents (such as other chlorhexidine vehicles, e.g. dentifrice-containing chlorhexidine, or other antimicrobial agents (e.g. cetylpyridinium chloride (CPC)) that the comparator arm/s did not receive because we would have been unable to separate the effect of the chlorhexidine from the effect of the other active agents;

- where the mechanical oral hygiene procedures were not the same in both the chlorhexidine mouthrinse and the comparator arms e.g. studies or study arms where experimental or comparator arms received more intensive or more frequent professional mechanical cleaning or used additional mechanical cleaning aids e.g. dental floss as part of the intervention that was different from the other study arms;

- where chlorhexidine mouthrinse was applied locally e.g. with a brush or via subgingival irrigation.

\section{Types of outcome measures}

\section{Primary outcomes}

Gingivitis measured using any appropriate index/scale.

Only trials that reported gingivitis as an outcome were considered for inclusion in the review. The clinical features of gingivitis can be assessed non-invasively by visual assessment of colour, contour and gingival bleeding or invasively using an instrument to provoke bleeding or both (Lorenz 2009). The Gingival Index of Löe and Silness (GI) utilises both visual assessment and assessment of bleeding on provocation. It can be measured at specific sites using six index teeth (Löe and Silness 1963) or all teeth (Löe 1967). Each site is scored on a 0 to 3 increasing scale and an average score can be calculated for each tooth assessed. The mean score for an individual represents an average score for the areas examined. The Modified Gingival Index (MGI) (Lobene 1986) can be used as a fullmouth index or applied to selected teeth and is measured non- invasively on a 0 to 4 increasing scale i.e. there is no attempt to use pressure to elicit bleeding. Many indices measure gingivitis solely by assessing bleeding on provocation but the techniques used to elicit bleeding can vary between indices. The Gingival Bleeding Index (Ainamo and Bay 1975) uses a blunt pocket probe to gently probe the orifice of the gingival crevice. If bleeding occurs within about 10 seconds after testing, a positive finding is recorded. The percentage of sites with gingival bleeding are calculated for the individual.

It is not uncommon for multiple measures of gingivitis to be presented within one study. Therefore the following hierarchy was developed to simplify data extraction.

- Where gingival inflammation and gingival bleeding were reported within the same study we extracted data on both outcomes.

- Where gingival inflammation was reported using more than one index within a study, the GI (Löe and Silness 1963; Löe 1967) was chosen over other indices and the MGI (Lobene 1986) was chosen next if the gingival index was not reported.

- Where gingival bleeding was reported within a study as bleeding on probing (BOP) (Ainamo and Bay 1975) and the proportion of sites with BOP using the GI (i.e. GI scores 2 or 3 ) (Löe and Silness 1963; Löe 1967), BOP was chosen over the proportion of sites with $\mathrm{BOP}$ ( $\mathrm{GI}$ scores 2 or 3 ).

Within the gingival index, the severity (the average extent of the disease) was considered a measure of gingival inflammation and occurrence (the proportion of sites with BOP i.e. GI scores 2 or 3 ) was considered a measure of gingival bleeding.

Outcome data at 4 to 6 weeks, 7 to 12 weeks, 13 weeks to 6 months and greater than 6 months were collected and reported but the primary analysis of the gingivitis data was concerned with shortterm use ( 4 to 6 weeks), along with a longer term measure at 6 months.

\section{Secondary outcomes}

Data on the following secondary outcomes were extracted from eligible trials that also reported gingivitis as an outcome.

- Dental plaque measured using any appropriate index/scale. Although dental plaque can be measured using a variety of different indices, the Plaque Index (Silness and Löe 1964) and the Turesky modification of the Quigley and Hein index (TQH) (Turesky 1970) are commonly used to measure dental plaque. Both use an increasing scale to quantify the amount of plaque present. The Plaque Index uses a 0 to 3 increasing scale to quantify the amount of plaque on each surface (buccal, lingual, mesial and distal) of six index teeth. An average score for each tooth and for the individual can then be calculated. The TQH uses a 0 to 5 increasing scale to quantify the amount of plaque on the buccal and lingual surfaces of the teeth. An average score for the individual can then be calculated. Where dental plaque was measured using more than one index within a study; the Plaque Index (Silness and Löe 1964) was chosen over other indices and the TQH (Turesky 1970) was chosen next if the Plaque Index was not reported.

- Calculus measured using any appropriate index/scale.

- Tooth staining measured using any appropriate index/scale. 
- Adverse effects (e.g. mucosal desquamation, taste disturbances, allergic reactions).

\section{Search methods for identification of studies}

\section{Electronic searches}

Cochrane Oral Health's Information Specialist conducted systematic searches in the following databases for randomised controlled trials and controlled clinical trials without language or publication status restrictions:

- Cochrane Oral Health's Trials Register (searched 28 September 2016) (Appendix 1);

- Cochrane Central Register of Controlled Trials (CENTRAL; 2016, Issue 8) in the Cochrane Library (searched 28 September 2016) (Appendix 2);

- MEDLINE Ovid (1946 to 28 September 2016) (Appendix 3);

- Embase Ovid (1980 to 28 September 2016) (Appendix 4);

- CINAHL EBSCO (Cumulative Index to Nursing and Allied Health Literature; 1937 to 28 September 2016) (Appendix 5).

Subject strategies were modelled on the search strategy designed for MEDLINE Ovid. Where appropriate, they were combined with subject strategy adaptations of the highly sensitive search strategy designed by Cochrane for identifying randomised controlled trials and controlled clinical trials as described in the Cochrane Handbook for Systematic Reviews of Interventions Chapter 6 (Lefebvre 2011).

\section{Searching other resources}

We searched the following trial registries for ongoing studies:

- US National Institutes of Health Ongoing Trials Register ClinicalTrials.gov (clinicaltrials.gov; searched 28 September 2016) (Appendix 6);

- World Health Organization International Clinical Trials Registry Platform (apps.who.int/trialsearch; searched 28 September 2016) (Appendix 7).

We searched the reference lists of included studies and relevant systematic reviews for further studies. We attempted to contact companies that manufacture chlorhexidine mouthrinse (Colgate Palmolive (Periogard), 3M (Peridex), GlaxoSmithKline (Corsodyl)) and companies that manufacture other types of mouthrinse (Johnson \& Johnson (Listerine), P\&G (Oral B brand)) to identify unpublished and ongoing studies.

We did not perform a separate search for adverse effects of interventions. Only the adverse effects that were described in the included studies were considered.

\section{Data collection and analysis}

\section{Selection of studies}

Two review authors independently and in duplicate screened the titles and abstracts of the list of studies identified by the searching process against the inclusion criteria for the review to identify eligible and potentially eligible studies. We obtained full-text copies of all the potentially eligible studies, and also of studies with insufficient information in the title/abstract to make a decision on eligibility. The review authors were not blinded to the journal name, authors, institution, results or sources of funding when assessing eligibility of the identified studies. Two review authors independently and in duplicate assessed the full-text copies to identify those that met the inclusion criteria. We contacted study authors for clarification or missing information relating to eligibility where necessary and feasible. We linked multiple reports of the same study together under one single study title. We resolved any disagreements on eligibility through discussion but, if this was not possible, an experienced member of the Cochrane Oral Health editorial team ( $\mathrm{H}$ Worthington) was consulted to achieve consensus. We recorded any studies failing to meet the inclusion criteria, along with reasons for exclusion.

The eligibility of the non-English language reports were assessed by two review authors independently and in duplicate. Relevant sections of the reports were translated with the assistance of Cochrane Oral Health. Non-English language reports that met the inclusion criteria for the review were translated in full.

This process is summarised in the 'Study flow diagram' (Figure 1). 
Figure 1. Study flow diagram.

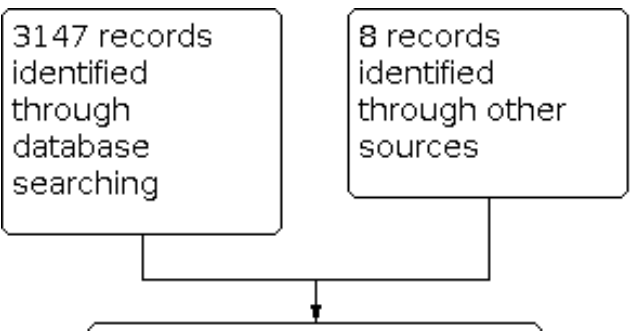

1520 records after

duplicates removed

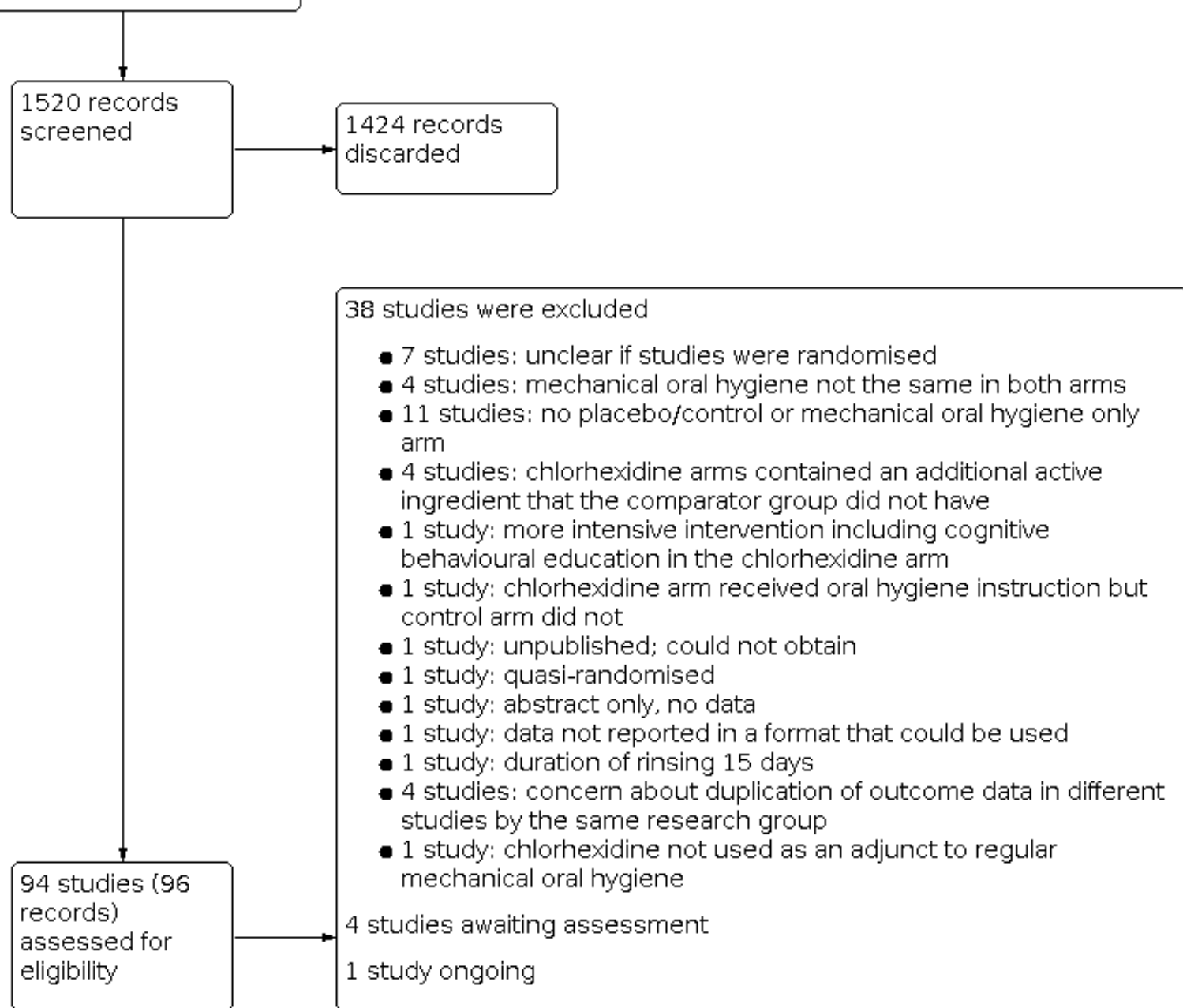


Figure 1. (Continued)

45 studies
included in
quantitative
synthesis
(meta-analysis)

\section{Data extraction and management}

Two review authors independently and in duplicate extracted data from the included studies using a customised Excel spreadsheet that was piloted on a small sample of studies. We contacted study authors for clarification or missing information where necessary and feasible. We resolved any disagreements through discussion and where agreement could not be reached, an experienced member of the Cochrane Oral Health editorial team $(\mathrm{H}$ Worthington) was consulted to achieve consensus.

We recorded the following data for each included study, which were then tabulated in the 'Characteristics of included studies' tables.

- Trial design, location/setting, number of centres, study duration.

- Details of the participants including demographic characteristics, criteria for inclusion and exclusion, and relevant information on gingivitis levels at baseline, numbers randomised to each study arm, and numbers analysed in each arm.

- Details of the type of experimental/comparator intervention, concentration and volume of mouthrinse, frequency and duration of rinsing, baseline prophylaxis (scale and polish), details of oral hygiene instruction $(\mathrm{OHI})$, supervision of mouthrinsing, timing of mouthrinsing in relation to toothbrushing and postrinsing instructions.

- Details of the outcomes reported, including method and timing of assessment.

- Sample size calculations, source of study funding, information about adverse effects, proportion of smokers in the studies/ study arms and declarations/conflicts of interest.

- Outcome data: For gingivitis and plaque we extracted outcome data from the end point of each study with the exception of studies where the duration of rinsing was longer than 6 months. In this situation interim data for gingivitis and plaque at 6 months were extracted in addition to data at the end point of the study. We considered interim data on extrinsic tooth staining and calculus formation important in determining the time of onset of these adverse effects. Therefore, where they were reported, data on extrinsic tooth staining and calculus formation were extracted at interim time points as well as at the end point of each study. Outcome data were grouped according the following time intervals: 4 to 6 weeks, 7 to 12 weeks, 13 weeks to 6 months and greater than 6 months. Where data on extrinsic tooth staining or calculus were reported twice within one time interval, the later results were data extracted. Where an outcome was measured after rinsing has ceased, we considered the time point that rinsing ceased as the primary end point. Studies that measured gingivitis and plaque outcomes only after cessation of rinsing were included in a separate analysis of the long-term effects of rinsing with chlorhexidine mouthrinse on gingivitis and plaque. We did not extract extrinsic tooth staining and calculus data reported after rinsing had ceased.

- Where studies reported mean scores for the Gingival Index at 4 to 6 weeks or 6 months but did not report a measure of variance and a measure of variance could not be obtained from the authors of the studies, the variance was estimated from the standard deviations reported in similar trials that used the same index at the same time point as described in Chapter 16 of the Cochrane Handbook for Systematic Reviews of Interventions (Higgins 2011). Where mean scores and measures of variance were reported only in graphs and the data could not be obtained from the authors of the studies, data were estimated by reading the data off the graphs in the published report for inclusion in meta-analyses. The graphs were enlarged and the data were estimated independently and in duplicate. Any disagreements were resolved by discussion. A sensitivity analysis was conducted to gauge the effects of estimating these outcome data on the overall results.

\section{Assessment of risk of bias in included studies}

Two review authors assessed the risk of bias of all included studies, independently and in duplicate, using Cochrane's domain-based, two-part tool as described in Chapter 8 of the Cochrane Handbook for Systematic Reviews of Interventions (Higgins 2011). We contacted study authors for clarification or missing information where necessary and feasible. We tried to resolve any disagreements on risk of bias through discussion but, if this was not possible, an experienced member of the Cochrane Oral Health editorial team $(\mathrm{H}$ Worthington) was consulted to achieve consensus. A 'Risk of bias' table was completed for each included study. For each domain of risk of bias, we first described what was reported to have happened in the study in order to provide a rationale for the second part, which involved assigning a judgement of 'low risk' of bias, 'high risk' of bias, or 'unclear risk' of bias.

For each included study, we assessed the following seven domains of risk of bias.

- Random sequence generation (selection bias): use of simple randomisation (e.g. random number table, computer-generated randomisation, central randomisation by a specialised unit), restricted randomisation (e.g. random permuted blocks), stratified randomisation and minimisation were assessed as at low risk of bias. Other forms of simple randomisation, such as repeated coin tossing, throwing dice or dealing cards, were also considered as at low risk of bias. If a study report used the phrase 'randomised' or 'random allocation' but with no further information, and if randomisation could not be confirmed, we assessed it as unclear for this domain.

- Allocation concealment (selection bias): use of centralised/ remote allocation, pharmacy-controlled randomisation (i.e. allocation of sequentially numbered mouthrinse containers 
of identical appearance) and sequentially numbered, sealed, opaque envelopes were assessed as at low risk of bias. If a study report did not mention allocation concealment, we assessed it as unclear for this domain.

- Blinding of participants and personnel (performance bias): if blinding was not mentioned, we assessed this domain as at unclear risk of bias. We only considered personnel blinding for studies with supervised mouthrinsing. If a study was described as double blind, we assumed that the intention was for participants and outcome assessors to be blinded to group allocation, however due to the propensity for chlorhexidine to stain the teeth and oral tissues and the association of its use with visible adverse effects such as mucosal desquamation and mucosal lesions, we considered the potential effect of these on blinding as follows.

- Where tooth staining/adverse effects were reported narratively or measured using a scale (where relevant) and were higher in the chlorhexidine rinse arm/s, we considered that participants could have worked out which study arm they were in and this could have affected their oral health behaviours and hence the outcome and assessed this domain as at high risk of bias irrespective of whether participant blinding was indicated.

- Where tooth staining/adverse effects were reported narratively or measured using a scale (where relevant) but were not higher in the chlorhexidine rinse arm $/ \mathrm{s}$ and participant blinding was also indicated, we assessed this domain as at low risk of bias.

- Where tooth staining/adverse effects were not reported, although we considered it likely in this situation that tooth staining or other visible adverse effects could have occurred and been noticeable in the chlorhexidine rinse arm/s after 4 weeks or more of rinsing, we assessed this domain as unclear as there was not enough information upon which to base a judgement.

- Where it was not possible to blind participants and personnel due to differences in the experimental and comparator interventions this domain was assessed as at high risk of bias.

- Blinding of outcome assessment (detection bias): if blinding was not mentioned, we assessed this domain as at unclear risk of bias. If a study was described as double blind, we assumed that the intention was for participants and outcome assessors to be blinded to group allocation, however due to the propensity for chlorhexidine to stain the teeth and oral tissues and the association of its use with visible adverse effects such as mucosal desquamation and mucosal lesions, we considered the potential effect of these on blinding as follows.

- Where tooth staining/adverse effects such as mucosal desquamation and mucosal lesions that could be visible to the outcome assessor were reported narratively or measured using a scale (where relevant) and were higher in the chlorhexidine arm/s, we considered that outcome assessors could have worked out which study arm participants were in and therefore could not be adequately blinded. In this situation we assessed this domain as at high risk of bias irrespective of whether blinding of outcome assessment was indicated.
- Where tooth staining/adverse effects such as mucosal desquamation and mucosal lesions that could be visible to the outcome assessor were reported narratively or measured using a scale (where relevant) but were not higher in the chlorhexidine arm/s and blinding of outcome assessment was also indicated, we assessed this domain as at low risk of bias.

- Where tooth staining/adverse effects such as mucosal desquamation and mucosal lesions that could be visible to the outcome assessor were not reported, although we considered it likely that tooth staining or other visible adverse effects could have occurred and been noticeable in the chlorhexidine arm/s after 4 weeks of more of rinsing, we assessed this domain as unclear as there was not enough information upon which to base a judgement.

- Incomplete outcome data (attrition bias): if $10 \%$ or less of randomised participants were excluded from the analysis, we assessed this as at low risk of bias. However, when attrition was greater than $10 \%$, assuming the missing participants in one group had a higher mean (e.g. gingivitis score) than those in the other group, as the attrition rate increased, so would the mean difference (MD) between groups, as described in Chapter 8 of the Cochrane Handbook for Systematic Reviews of Interventions (Higgins 2011). This situation led to a judgement of high risk of bias if we believed that the attrition was high enough to have resulted in a distortion of the true intervention effect, or if there was considerably greater attrition in one group than another. If attrition was greater than $10 \%$, but with the additional factors of not being reported by group and insufficient reporting of reasons for attrition, this led to a judgement of unclear risk of bias. If it was not clear from the study report how many participants were randomised into each group, we assessed it as at unclear risk of bias for this domain.

- Selective reporting (reporting bias): if the study either reported outcomes not stated a priori in the methods section or the study protocol (if available) or did not report outcomes stated in the methods section, we assessed this as at high risk of bias. If gingivitis and plaque outcomes were reported with insufficient information to allow us to use the outcome data in a metaanalysis (e.g. no information on variance), we assessed it as at high risk of bias. We considered the reporting of adverse effects associated with the use of chlorhexidine mouthrinse to be important outcomes. For an assessment of low risk of bias we required either a statement that there were no adverse effects associated with the chlorhexidine rinse or if present, that adverse effects be reported by group. Where tooth staining was not reported or was measured using an index but insufficient data were reported to allow us to use the data in a meta-analysis we assessed it as at high risk of bias. Where tooth staining was not measured using an index but its presence/absence was reported we assessed this as at low risk of bias, provided that the information was reported for all participants by group.

- Other bias: any other potential source of bias that may feasibly alter the magnitude of the effect estimate (e.g. baseline imbalances in potentially important prognostic factors between intervention groups and differential diagnostic activity by outcome assessors).

We summarised the risk of bias as follows. 


\begin{tabular}{llll}
\hline Risk of bias & Interpretation & In outcome & In included studies \\
\hline Low risk of bias & $\begin{array}{l}\text { Plausible bias unlikely to seri- } \\
\text { ously } \\
\text { alter the results }\end{array}$ & $\begin{array}{l}\text { Low risk of bias for all key } \\
\text { domains }\end{array}$ & $\begin{array}{l}\text { Most information is from studies at low risk of } \\
\text { bias }\end{array}$ \\
\hline Unclear risk of bias & $\begin{array}{l}\text { Plausible bias that raises some } \\
\text { doubt about the results }\end{array}$ & $\begin{array}{l}\text { Unclear risk of bias for } \\
\text { one or more key domains }\end{array}$ & $\begin{array}{l}\text { Most information is from studies at low or un- } \\
\text { clear risk of bias }\end{array}$ \\
\hline High risk of bias & $\begin{array}{l}\text { Plausible bias that seriously } \\
\text { weakens confidence in the re- } \\
\text { sults }\end{array}$ & $\begin{array}{l}\text { High risk of bias for one } \\
\text { or more key domains }\end{array}$ & $\begin{array}{l}\text { The proportion of information from studies at } \\
\text { high risk of bias is sufficient to affect the inter- } \\
\text { pretation of results }\end{array}$ \\
\hline
\end{tabular}

\section{Measures of treatment effect}

We used the methods outlined in the Cochrane Handbook for Systematic Reviews of Interventions (Higgins 2011). We combined mean differences (MDs) where studies used the same scale and standardised mean differences (SMDs) where studies used different scales for continuous outcomes. For dichotomous outcomes, we reported risk ratios (RR) and 95\% confidence intervals.

\section{Unit of analysis issues}

The participant was the unit of analysis. We were unable to use data from studies that reported number of sites rather than number of individuals in the meta-analyses, as failure to take the clustering into account could lead to an overestimate of effect, with narrower confidence intervals and smaller $P$ values. Had we included clusterRCTs we would have analysed the results taking account of the clustering present in the data, otherwise we would have used the methods outlined in Section 16.3.4 of the Cochrane Handbook for Systematic Reviews of Interventions in order to perform an approximately correct analysis (Higgins 2011).

\section{Dealing with missing data}

We attempted, where feasible, to contact the authors of studies to obtain missing data or for clarification. Where appropriate, we used the methods outlined in Chapter 16 of the Cochrane Handbook for Systematic Reviews of Interventions in order to estimate missing standard deviations for the main outcome only (gingivitis) (Higgins 2011). We did not use any further statistical methods or carry out any further imputation to account for missing data. Where mean scores and measures of variance were only reported in graphs and could not be obtained from the authors of the studies, data were estimated by reading the data off the graphs in the published report for inclusion in meta-analyses. The graphs were enlarged and the data were estimated independently and in duplicate. Any disagreements were resolved by discussion.

\section{Assessment of heterogeneity}

If meta-analyses were performed, we assessed the possible presence of heterogeneity visually by inspecting the point estimates and confidence intervals on the forest plots; if the confidence intervals had poor overlap then heterogeneity was considered to be present. We also assessed heterogeneity statistically using a $\mathrm{Chi}^{2}$ test, where a $\mathrm{P}$ value $<0.1$ indicated statistically significant heterogeneity. Furthermore, we quantified heterogeneity using the $\mathrm{I}^{2}$ statistic. A guide to interpretation of the $1^{2}$ statistic given in Section 9.5.2 of the Cochrane Handbook for Systematic Reviews of Interventions is as follows (Higgins 2011):

- $0 \%$ to $40 \%$ : might not be important;

- 30\% to $60 \%$ : may represent moderate heterogeneity;

- $50 \%$ to $90 \%$ : may represent substantial heterogeneity;

- $75 \%$ to $100 \%$ : considerable heterogeneity.

\section{Assessment of reporting biases}

Assessment of reporting bias within studies has already been described in the section Assessment of risk of bias in included studies.

Reporting biases can occur when reporting (or not reporting) research findings is related to the results of the research (e.g. a study that did not find a statistically significant difference/result may not be published). Reporting bias can also occur if ongoing studies are missed (but that may be published by the time the systematic review is published), or if multiple reports of the same study are published, or if studies are not included in a systematic review due to not being reported in the language of the review authors. We attempted to limit reporting bias in the first instance by conducting a detailed, sensitive search, including searching for ongoing studies, and any studies not reported in English were translated. If there were more than 10 studies included in a metaanalysis for the primary outcome (gingivitis), we assessed the possible presence of reporting bias by testing for asymmetry in a funnel plot. We carried out statistical analysis using the methods described by Egger 1997 for continuous outcomes.

\section{Data synthesis}

We carried out a meta-analysis only where studies of similar comparisons reported the same outcomes at the same time interval. We combined mean differences (MDs) where studies used the same scale and standardised mean differences (SMDs) where studies used different scales for continuous outcomes. For dichotomous outcomes, we reported risk ratios (RR) and 95\% confidence intervals. Due to anticipated heterogeneity we used random-effects models for all meta-analyses.

We reported the results from studies not suitable for inclusion in a meta-analysis both in the results and in additional tables.

\section{Subgroup analysis and investigation of heterogeneity}

Where there were sufficient studies, we carried out the following subgroup analyses. 
- Baseline prophylaxis (scale and polish) versus none.

- Children versus adults.

- Different chlorhexidine concentrations.

- Initial levels of gingivitis.

- Gingivitis only versus gingivitis + periodontitis.

\section{Sensitivity analysis}

In order to ensure our conclusions were robust, we carried out sensitivity analyses (where there were sufficient studies for each outcome) by excluding studies where outcome data were read from graphs, studies where standard deviations were estimated and studies where periodontal treatment was conducted during the study.

We would have carried out sensitivity analyses by excluding studies at high and unclear risk of bias, however this was not possible as apart from one study at unclear risk of bias, all of the included studies were at high risk of bias.

\section{Summary of findings and assessment of the quality of the evidence}

We produced a 'Summary of findings' table for chlorhexidine mouthrinse compared to placebo/control mouthrinse or no mouthrinse for the following outcomes: gingivitis measured using the Gingival Index at 4 to 6 weeks, plaque at 4 to 6 weeks, tooth staining at 4 to 6 weeks and other adverse effects. We used GRADE methods (GRADE 2004), and the GRADEpro GDT online tool for developing 'Summary of findings' tables (www.guidelinedevelopment.org). We assessed the quality of the body of evidence for each outcome by considering the overall risk of bias of the included studies, the directness of the evidence, the inconsistency of the results, the precision of the estimates, and the risk of publication bias. We categorised the quality of each body of evidence as high, moderate, low, or very low.

\section{RE S U L T S}

\section{Description of studies}

\section{Results of the search}

The searches resulted in 1520 references after de-duplication. Two review authors screened the titles against the inclusion criteria for this review, independently and in duplicate, discarding 1424 references in the process. We obtained full-text copies of the remaining 96 references ( 94 studies) and examined them independently and in duplicate, excluding 38 studies at this stage. A further four studies are awaiting assessment of their eligibility to be included in the review and one study is ongoing. Therefore, fiftyone studies ( 53 records) met the inclusion criteria for this review. This process is presented diagrammatically in Figure 1.

\section{Included studies}

\section{Characteristics of the trial designs and settings}

Fifty-one studies met the inclusion criteria for this review. All studies were of parallel-group design. Sixteen studies had two study arms and compared chlorhexidine rinse with placebo rinse. However, thirty-five studies had multiple study arms and not all study arms were relevant to this review. Twenty-two studies had three arms; 10 had four arms and two had five arms. One of the studies with four study arms (Flotra 1972) had three chlorhexidine rinse groups and one placebo rinse group but presented data only for two groups: the three chlorhexidine study arms combined and the placebo group. One additional study (Feres 2012) had three main therapeutic arms which were each divided into chlorhexidine rinse and placebo rinse groups using a $2 \times 3$ factorial design.

Sixteen studies were conducted in the USA, six in Brazil, five in the UK, four in Germany, three in India, three in Sweden, two in each of the following countries: Canada, Mexico and Spain, and one in each of the following countries: Argentina, China, Iran, Italy, Norway, Switzerland, the Netherlands and Turkey. The settings of the studies were diverse and often poorly reported. Twenty-seven studies were conducted in a dental school/university setting and an additional three studies were assumed, based on the information provided, to have been conducted in a dental school/university setting (Emling 1992; Pereira 2011; Van Strydonck 2008). Two studies were conducted under "dental office conditions" (Grossman 1986; Grossman 1989), two in general dental practices (Eaton 1997; Fine 1985) and one in a private practice limited to periodontics (Sanz 1989). Three studies were conducted in a school setting (de la Rosa 1988; de la Rosa 1988b; Jayaprakash 2007). The remaining studies were conducted in military garrisons (Flotra 1972; Hase 1998); pensioners/nursing homes (Lopez-Jornet 2012; Weitz 1992); a bone marrow transplant unit (Ferretti 1987) and a knitting factory (Corbet 1997). Three studies were conducted at what we assumed were clinical research facilities (Charles 2004; Jose 2015; Stookey 2005) and in four studies the setting of the study was not clear (Flemmig 1990; Hase 1995; Sanz 1994; Taller 1993). The numbers of centres involved in the studies was often unclear but it appeared that all but two of the studies were conducted at a single centre. One study (Eaton 1997) was conducted at five suburban dental practices in Southern England and another study (Jose 2015) was conducted at two sites (Manchester and Wirral) in the UK.

In 19 studies direct support from industry was explicitly stated. Nine studies received support from P\&G; three from GSK; two from Teledyne and one from each of the following companies: GABA, J\&J, Oral B, Smithkline Beecham and Warner Lambert. Three studies that did not appear to be funded directly by industry explicitly stated that oral care products used in the study had been provided by industry (Anderson 1997; Sanz 1994; Stookey 2005). Three studies (Hase 1995; Hase 1998; Lang 1998) were associated with Biosurface Pharma AB through authorship and another study was associated with 'Dental Products Testing' and Pfizer through authorship (Charles 2004). Of the remaining studies that reported a source of support, two appeared to be funded by universities (Rahmani 2006; Taller 1993); six appeared to be funded by research grants (Anauate-Netto 2014; Bajaj 2011; Corbet 1997; Faveri 2006; Feres 2009; Feres 2012); and in 17 studies there was no statement regarding funding or support. Only one study (Zimmer 2015) stated that the conduct of the research and final decisions regarding the study report were independent of the funder (GSK).

Only 13 of the studies described a sample size calculation. For six of these studies (Faveri 2006; Feres 2009; Feres 2012; Graziani 2015; Turkoglu 2009; Van Strydonck 2008) the sample size required was reported and achieved but for three of these studies the sample size was based on pocket probing depth (PPD) (Faveri 2006; Feres 2012) and clinical attachment loss (CAL) (Feres 2009) rather than gingivitis. Two studies reported the sample size calculation and number of participants required in each group but the minimum 
sample size was not achieved at the end of the studies (Hase 1998; Lang 1998). Five of the studies (Axelsson 1987; Brightman 1991; Charles 2004; Eaton 1997; Zimmer 2015) described the sample size calculation but not the required sample size so we were unable to determine if the required sample size was achieved. In one study the sample size calculation was not described but the required sample size was reported and achieved (Bhat 2014).

\section{Characteristics of the participants}

A total of 6995 participants provided data for the included studies. The total number of participants providing data for the study arms relevant to this review (excluding the irrelevant study arms) was 5345 with the numbers analysed in each study ranging from 20 to 852.

Six studies involved children and adolescents (Anderson 1997; Bajaj 2011; Brightman 1991; de la Rosa 1988; de la Rosa 1988b; Jayaprakash 2007). The age range of the children was 8 to 16 and three of these studies also included some young adults aged 17 and 18 (Brightman 1991; de la Rosa 1988; de la Rosa 1988b). Four studies while predominantly focused on adults, included children and adolescents among the participants: in Axelsson 1987 participants were aged 16 to 50 years old; in Fine 1985no participants under the age of 12 were included; and in Navarro 1998 the age range of participants was 14 to 35 years old. In Ferretti 1987, a study involving individuals undergoing bone marrow transplantation and chemoradiotherapy, the age range was 5 to 51 years old. The remaining forty-one studies involved adults. The age range of adult participants was 17 to 94 years with the mean age ranging from 20 to 83 .

Twenty-two studies had a greater proportion of females than males and in four studies, the proportion of males was greater (Lang 1998; Southern 2006; Stookey 2005; Taller 1993). In five studies there was an equal balance of males to females (Bhat 2014; Graziani 2015; Pereira 2011; Turkoglu 2009; Zimmer 2006). Five studies involved male participants only (de la Rosa 1988; de la Rosa 1988b; Hase 1995; Hase 1998; Navarro 1998) and in the remaining 15 studies, the proportion of males to females was not reported. Eight studies included smokers among the participants (Charles 2004; Eaton 1997; Ernst 2005; Jose 2015; Joyston-Bechal 1993; Overholser 1990; Zimmer 2006; Zimmer 2015) with the proportion of smokers in each study ranging from $11 \%$ to $41 \% .10$ studies excluded smokers and in the remaining 33 studies, the smoking status of the participants was not reported.

Twenty-four studies reported gingivitis at baseline using the Gingival Index of Löe and Silness (Löe and Silness 1963; Löe 1967). In one study involving dental students (Lucas 1999) where normal gingiva were obtained in a pre-experimental phase by scaling and polishing and twice daily brushing, the mean baseline gingivitis score was zero. In two other studies (Navarro 1998; Jayaprakash 2007) gingivitis at baseline was particularly low, mean gingival index scores were 0.049 and 0.057 respectively. In the remaining 21 studies the mean gingival index ranged from 0.53 to 1.87 with an overall mean of 1.09

In many of the included studies, the periodontal status of the participants was not explicitly described. In 15 studies, the participants appeared to have gingivitis but no periodontitis whereas in nine studies, the participants had periodontitis of varying severity in addition to gingivitis (Chaves 1994; Corbet
1997; Ernst 2005; Faveri 2006; Feres 2009; Feres 2012; Graziani 2015; Rahmani 2006; Sanz 1989). In three of these studies the periodontitis was untreated at baseline (Faveri 2006; Feres 2009; Feres 2012) and in one study the participants required osseous periodontal surgery (Sanz 1989). In 16 studies it was unclear whether the participants had periodontitis in addition to gingivitis but it was deemed likely that at least some of the participants in these studies had periodontitis; two studies involved older adults (Lopez-Jornet 2012 (aged > 65 years) and Weitz 1992 (mean age 83)) and in the remaining 14 studies, the inclusion criteria allowed for the participants to have deep pockets and clinical attachment loss but the periodontal status of the people actually included in the study was not described. In 11 studies the participants clearly had gingivitis at baseline, but it was unclear whether the participants also had periodontitis.

\section{Characteristics of the interventions}

Nine studies compared chlorhexidine rinse and mechanical oral hygiene with mechanical oral hygiene alone.Two of these studies had additional chlorhexidine mouthrinse arms that were also included in the analysis (Jose 2015; Zimmer 2015). All of these studies except Jose 2015 also had additional arms that were not relevant to this review. Forty-two studies compared chlorhexidine rinse with placebo or control rinse. Six of these studies had additional chlorhexidine mouthrinse arms. Data from five of these six studies were included in the analysis (Flotra 1972; Graziani 2015; Hoffmann 2001; Jayaprakash 2007; Overholser 1990; Segreto 1986). We could not include data from the three separate chlorhexidine arms for Flotra 1972 because the authors combined the three arms into one experimental group. Twenty of these 42 studies comparing chlorhexidine rinse with placebo or control rinse also had additional arms that were not relevant to this review.

Of the studies with one concentration of chlorhexidine mouthrinse, 10 evaluated $0.2 \%$ chlorhexidine mouthrinse; 29 evaluated $0.12 \%$ chlorhexidine mouthrinse; two evaluated $0.1 \%$ chlorhexidine mouthrinse (Bajaj 2011; Ernst 2005) and four evaluated 0.05\% or $0.06 \%$ chlorhexidine mouthrinse (Jayaprakash 2007; JoystonBechal 1993; Zimmer 2006; Zimmer 2015). Four studies had additional arms evaluating different chlorhexidine concentrations (Axelsson 1987; Flotra 1972; Hoffmann 2001; Segreto 1986) and in two studies, the concentration of the chlorhexidine rinse was not reported (Fine 1985; Turkoglu 2009).

Most of the studies evaluating $0.2 \%$ chlorhexidine mouthrinse used a volume of $10 \mathrm{~mL}$ twice daily for one minute whereas most of the studies evaluating $0.1 \%$ or $0.12 \%$ chlorhexidine mouthrinse used a volume of $15 \mathrm{~mL}$ twice daily. Duration of rinsing for the studies of $0.1 \%$ and $0.12 \%$ chlorhexidine mouthrinse varied from 30 seconds to 1 minute but most of the studies employed a 30 -second rinse. The studies of $0.05 \%$ and $0.06 \%$ chlorhexidine mouthrinse predominantly used a volume of $10 \mathrm{~mL}$ once or twice a day for 30 seconds. In some studies with multiple arms different volumes of rinse were used in different arms: in three studies the placebo/control arms and other non-chlorhexidine active rinse groups rinsed with $20 \mathrm{~mL}$ while the chlorhexidine arms rinsed with $10 \mathrm{~mL}$ (Axelsson 1987) or $15 \mathrm{~mL}$ (Charles 2004; Overholser 1990); in another study the $0.1 \%$ chlorhexidine arm rinsed with $15 \mathrm{~mL}$ while the two $0.06 \%$ chlorhexidine arms used $10 \mathrm{~mL}$ of rinse (Hoffmann 2001). 
In six studies the volume of rinse used by participants was not reported. In three of these studies participants rinsed twice daily but rinse volume and duration were not reported (Chaves 1994; Rahmani 2006; Sanz 1994). In two of the studies, participants rinsed once a day for 30 seconds with an unknown volume of rinse (Fine 1985; Zimmer 2006). In another study rinse volume, frequency and duration were not reported (Emling 1992).

Of the nine studies that compared chlorhexidine rinse and mechanical oral hygiene with mechanical oral hygiene alone, six involved regular mechanical cleaning using a toothbrush and toothpaste (Chaves 1994; Flemmig 1990; Jose 2015; Taller 1993; Zimmer 2006; Zimmer 2015). In one study floss and wood points were also used by participants (Fine 1985). In another study participants underwent conventional ultrasonic debridement (Rahmani 2006) and were given interdental cleaning aids, and participants in a further study used a toothbrush and no toothpaste (Van Strydonck 2008).

Thirty-five of the 51 studies compared chlorhexidine mouthrinse with placebo and seven studies compared chlorhexidine mouthrinse with control. The control rinses were $5 \%$ hydroalcohol solution (Charles 2004); coloured and flavoured 5\% hydroalcohol solution (Overholser 1990); 0.5\% benzene alcohol (Flotra 1972); saline (Bhat 2014; Graziani 2015) and water (Bajaj 2011; Hoffmann 2001). In one of these studies comparing $0.2 \%$ chlorhexidine mouthrinse with placebo rinse, participants were instructed to brush without toothpaste before using the mouthrinse (Hase 1995). In another study comparing $0.12 \%$ chlorhexidine mouthrinse with placebo rinse, all participants used a gum care dentifrice (Blend-amed) (Sanz 1994).

Thirty-six studies reported a baseline prophylaxis to remove plaque and thus assess the potential for chlorhexidine mouthrinse to inhibit plaque accumulation and its ability to reduce gingivitis. In two of the studies that reported a baseline prophylaxis, an additional prophylaxis was also provided 2 weeks before baseline (Brecx 1993; Hoffmann 2001). In another study the baseline prophylaxis was conducted during the first week of the study (Stookey 2005). Participants in eight of the nine studies in which some or all of the participants had periodontitis at baseline either had professional prophylaxis at baseline (Chaves 1994; Ernst 2005; Graziani 2015), or adjunctive professional non-surgical (Faveri 2006; Feres 2009; Feres 2012; Rahmani 2006) or surgical periodontal treatment (Sanz 1989) during the study. In the three studies where the participants with periodontitis had a professional prophylaxis at baseline only, scaling and polishing was supragingival (Graziani 2015) or the extent of the professional cleaning was not clear (Chaves 1994; Ernst 2005).

Participants in six studies did not have a baseline prophylaxis. These studies were thus designed to assess the potential for chlorhexidine mouthrinse to reduce plaque and gingivitis in individuals who do not obtain professional tooth cleaning or scaling prior to commencing mouthrinse use (Corbet 1997; Jayaprakash 2007; Jenkins 1993; Lopez-Jornet 2012; Southern 2006; Weitz 1992). One of these studies included participants with untreated periodontitis (Corbet 1997). Participants in this study received no professional prophylaxis and no oral hygiene instructions. In seven studies it was not clear whether a baseline prophylaxis had been carried out (Anauate-Netto 2014; Bajaj 2011; Bhat 2014; Emling 1992; Taller 1993; Turkoglu 2009; Zimmer 2015). In one study (Zimmer 2006), the screening examination was followed by calculus removal in the lower front teeth only. In another study, where it was not clear whether the participants had periodontitis in addition to gingivitis, there was no prophylaxis at baseline but supragingival scaling was performed at week 9 and subgingival scaling was performed after 13 weeks (Flotra 1972).

Twenty-two studies reported that participants received some form of oral hygiene instruction. Where details were reported about the oral hygiene instruction, it usually involved instruction about toothbrushing technique. In two studies, oral hygiene instruction included instruction in the use of interdental cleaning aids (i.e. interdental brushes or dental floss or both) (Chaves 1994; Graziani 2015) and in another three studies participants were given interdental cleaning aids; presumably instruction in their use was included in the oral hygiene instruction but this not clear from the text (Fine 1985; Rahmani 2006; Taller 1993). In two studies participants were advised on duration and frequency of brushing but not technique (Pereira 2011; Van Strydonck 2008) and in another study, participants were given brief instruction about using the toothbrush they were given but were not given any instructions about duration or technique of toothbrushing (Zimmer 2006).

The participants in 27 studies did not receive any oral hygiene instruction. In 18 of these studies participants were advised to continue their usual oral hygiene practices throughout the duration of the studies. The participants in one of these 18 studies (Ferretti 1987) were undergoing bone marrow transplant and chemoradiotherapy and their usual oral hygiene regimen involved oral cleaning with a foam rubber toothbrush substitute and saline solution irrigations. In another of these studies where continuation of usual oral hygiene practices was advised, it was explicitly stated that use of interproximal cleaning devices was permitted if they were part of participants' usual oral hygiene routine (Zimmer 2015). In two studies it was not clear whether participants received oral hygiene instruction (Bajaj 2011; Stookey 2005). In one of these studies toothbrushing was supervised Monday to Friday in the mornings but it is not clear if oral hygiene instructions were provided to participants (Stookey 2005).

In 38 studies mouthrinsing was unsupervised. Mouthrinsing was fully supervised in only three studies, all conducted in a school setting (de la Rosa 1988; de la Rosa 1988b; Jayaprakash 2007). In 10 studies mouthrinsing was partially supervised for practical reasons. In five of the studies mouthrinsing was fully supervised on weekdays only (Axelsson 1987; Bajaj 2011; Bhat 2014; Lang 1998; Overholser 1990); in another two studies, mouthrinsing was supervised once a day on weekdays only (Charles 2004; Stookey 2005). In another three studies the mouthrinsing was supervised except for two weeks during the Chinese New Year (Corbet 1997); after participants were discharged from hospital (Ferretti 1987) and when participants were at military training (Hase 1998).

Thirty studies reported instructing participants to use the mouthrinse after toothbrushing. Three of these studies specified waiting 30 minutes after toothbrushing before using the mouthrinse (Feres 2012; Turkoglu 2009; Zimmer 2015). One of these studies specified waiting five timed minutes before mouthrinsing (Jose 2015). One study reported instructing participants to use mouthrinse at a separate time to toothbrushing (Charles 2004). In the remaining 20 studies the timing of mouthrinsing in relation to toothbrushing was not reported. 
In most of the studies the duration of the intervention (mouthrinsing with chlorhexidine) was 6 months or less broken down as follows: 4 to 6 weeks ( 15 studies); 2 months ( 9 studies); 10 weeks ( 1 study); 3 months (9 studies); 4 months ( 2 studies) or 6 months ( 13 studies). In two studies the duration of the intervention was longer than 6 months; one study of 9 months' duration (Bajaj 2011) and another of 24 months' duration (Banting 1989).

In most studies the final follow-up assessment was at the end of the intervention phase. However in four studies participants ceased mouthrinsing before the final outcome assessment. In these studies the final assessment of the outcomes was carried out 4 weeks (Faveri 2006; Ferretti 1987), 4 months (Feres 2009) and 10 months (Feres 2012) after mouthrinsing had ceased. For this review, we considered the time that mouthrinsing ceased to be the primary end point for data extraction.

\section{Characteristics of the outcomes}

\section{Gingivitis}

\section{Gingival inflammation}

Thirty-eight studies included gingival inflammation as an outcome. The most commonly used index for measuring gingival inflammation was the Gingival Index of Löe and Silness (GI) (Löe and Silness 1963; Löe 1967). The following indices were also used to measure gingival inflammation: the Modified Gingival Index (MGI) (Lobene 1986), the Papillary Bleeding Score (PBS) (Loesche 1979), and the Papillary Marginal Gingival Index (PMGI) (de la Rosa and Sturzenberger 1976). Thirty-one studies measured gingival inflammation using the GI; two studies used the MGI (Eaton 1997; Overholser 1990); one study used the PBS (Anauate-Netto 2014); and four studies used the PMGI (de la Rosa 1988; de la Rosa 1988b; Ferretti 1987; Segreto 1986). In one of these studies (Segreto 1986), the PMGI was measured with visual inspection only (no probing) in addition to the $\mathrm{Gl}$. One study reported a total mean score for a nonspecific gingival inflammation index (Fine 1985).

\section{Gingival bleeding}

Thirty-four studies included gingival bleeding as an outcome. The following indices were used to measure gingival bleeding: the $\mathrm{GI}$ (Löe and Silness 1963; Löe 1967) scores 2 and 3, the Gingival Bleeding Index of Ainamo and Bay (Ainamo and Bay 1975), the Eastman Interdental Bleeding Index (Caton and Polson 1985), the Bleeding Index/Bleeding on Marginal Probing (Saxton and van der Ouderaa 1989; van der Weijden 1994), a modification of the method described by Cowell et al (Cowell 1975), Papillary Bleeding Score (PBS) (proportion of sites with a PBS $\geq 2$ ) (Loesche 1979) and the Papillary Bleeding Index (Muhlemann 1977; Saxer 1975).

Thirteen studies reported gingival bleeding using scores 2 and 3 from the GI. One of these studies (Southern 2006) also reported bleeding on probing after 15 seconds. Another two studies (Hase 1998; Lang 1998) measured bleeding on probing and the method used suggests that it was based on scores 2 and 3 of the GI. Three studies (Graziani 2015; Pereira 2011; Rahmani 2006) measured gingival bleeding using the Gingival Bleeding Index of Ainamo and Bay. Two studies (Brightman 1991; Overholser 1990) used the Eastman Interdental Bleeding Index. Two studies (Ernst 2005; Van Strydonck 2008) reported the Bleeding Index/Bleeding on Marginal Probing. One study (Joyston-Bechal 1993) used a modification of the method described by Cowell et al. One study (AnauateNetto 2014) reported the proportion of sites with a PBS $\geq 2$ and three studies (Turkoglu 2009; Zimmer 2006; Zimmer 2015) used the Papillary Bleeding Index. A further seven studies measured the proportion of sites with bleeding on probing but were not specific about the index used (Chaves 1994; Faveri 2006; Feres 2009; Feres 2012; Flemmig 1990; Hase 1995; Taller 1993); three of these studies also reported the mean proportion of sites with gingival bleeding but were not specific about the index used (Faveri 2006; Feres 2009; Feres 2012).

\section{Plaque}

Forty-eight studies included plaque as an outcome. The majority of the studies measured dental plaque accumulation using the Plaque Index of Silness and Löe (Silness and Löe 1964) or the Turesky modification of the Quigley and Hein Index (TQH) (Turesky 1970). The following indices were also used to measure plaque: Soparker's modification of the TQH (Lobene 1982), the Modified Proximal Plaque Index (MPPI) (Zimmer 2005), the Quigley and Hein Plaque Index (Quigley 1962), the Approximal Plaque Index (API) (Lange 1977), a full-mouth plaque score (FMPS) (O'Leary 1972), and the method described by Greene and Vermilion (Greene and Vermillion 1964).

Twenty-one studies reported using the Plaque Index of Silness and Löe. Nineteen studies reported using TQH. One of these studies (Jose 2015) used Soparker's modification of the TQH, and one of these studies (Zimmer 2015) reported the MPPI in addition to $\mathrm{TQH}$. Two studies in the same report that used the TQH (de la Rosa 1988; de la Rosa 1988b) did not report any outcome data for plaque. One study (Zimmer 2006) reported both the MPPI and the Quigley and Hein Plaque Index. The following indices were reported in one study each: the Quigley and Hein Plaque Index (Turkoglu 2009), the API (Ernst 2005), FMPS (Graziani 2015), and the method described by Greene and Vermilion (Lucas 1999). Three studies (Faveri 2006; Feres 2009; Feres 2012) measured the presence or absence of plaque accumulation but were not specific about the index used.

\section{Adverse effects}

\section{Calculus}

Twelve studies reported calculus as an outcome. Seven studies (Banting 1989; Charles 2004; Emling 1992; Flemmig 1990; Hase 1998; Overholser 1990; Sanz 1994) reported using the VolpeManhold Calculus Index (Manhold 1965; Volpe 1965). Two studies (Corbet 1997; Lang 1998) reported using the Calculus Surface Index (CSI) (Ennever 1961). One study (Anderson 1997) reported using the Retention Index (Björby and Löe 1966; Löe 1967). One study (Turkoglu 2009) used a calculus index described in the National Institutes of Health (NIH) report 'Oral health of United States adults' (report not available online) and in one study the index used was not specified (Grossman 1986). In two additional studies accumulation of calculus in the chlorhexidine rinse groups was noted but not measured using an index or scale (de la Rosa 1988; de la Rosa 1988b).

\section{Extrinsic tooth staining}

Twenty-one studies included extrinsic tooth staining as an outcome but this was measured and reported in a variety of different ways. In 16 studies, the following indices were used to measure extrinsic tooth staining: the Discolouration Index (Lobene 1968), the Discolouration Index of Lang and Räber (Lang and Räber 1981), the Shaw and Murray Index (Shaw 1977), the Gingival Modification 
of the Lobene Stain Index (GMSI) (Grundemann 2000; Lobene 1968), and Meckels stain grading method (Lang 1982). Seven studies (Axelsson 1987; Charles 2004; Emling 1992; Ernst 2005; Joyston-Bechal 1993; Navarro 1998; Overholser 1990) reported the Lobene Discolouration Index. Five studies (Anderson 1997; Brecx 1993; Corbet 1997; Hoffmann 2001; Lang 1998) reported the Discolouration Index of Lang and Räber. One study (Hase 1998) reported the Shaw and Murray Index and another (Jenkins 1993) a modification of the Shaw and Murray Index. One study (Van Strydonck 2008) reported the GMSI and one study (Ferretti 1987) used Meckels stain grading method.

One study (Brightman 1991) reported using the Mean Stain Severity Index that was created at the Case Western Reserve University school of dentistry, to record intensity and area of stain. Another study (Graziani 2015) reported dividing the buccal surfaces of the eight central incisors into three areas: incisal, approximal and gingival according to Lobene 1968 and Grundemann 2000 and using a Staining Index (SI) to record the dichotomous presence or absence of staining in each area and to calculate the percentage of the total area showing staining. Three studies (Flemmig 1990; Grossman 1989; Sanz 1994) described their method of measuring tooth staining but did not specify a particular index.

Sixteen of the other included studies described information regarding tooth staining/discolouration/pigmentation reported by study participants (Anauate-Netto 2014; Bhat 2014; Feres 2009; Hase 1995; Pereira 2011); noted by the clinicians during the clinical examination but not measured using an index or scale (de la Rosa 1988; de la Rosa 1988b; Flotra 1972; Grossman 1986; LopezJornet 2012; Turkoglu 2009; Zimmer 2006; Zimmer 2015); or listed as a reason for participant withdrawal from the studies (Banting 1989; Eaton 1997). In one study (Sanz 1989) the presence or absence of tooth staining was assessed from clinical photographs of the mandibular facial anterior teeth. We included information regarding tooth staining from these studies in meta-analyses where possible but were unable to include participant-reported data, data where staining of teeth were reported combined with tongue staining or denture staining or where tooth staining was reported grouped with other adverse effects.

\section{Other adverse effects}

Fourteen studies did not report any adverse effects apart from extrinsic staining of teeth or oral tissues alone or both (Brecx 1993; Brightman 1991; Ferretti 1987; Grossman 1989; Hoffmann 2001; Joyston-Bechal 1993; Lopez-Jornet 2012; Overholser 1990; Van Strydonck 2008; Zimmer 2015) or with calculus (Corbet 1997; Flemmig 1990; Grossman 1986; Sanz 1994). Twenty-two studies reported at least one adverse effect relating to the chlorhexidine mouthrinse use apart from extrinsic tooth staining/staining of the oral tissues and calculus. Three studies (Chaves 1994; Feres 2009; Segreto 1986) mentioned a bitter or unacceptable taste associated with the chlorhexidine mouthrinse and no other adverse effects, except for one of the studies (Feres 2009) which also mentioned tooth staining. Adverse effects were not mentioned in six studies (Bajaj 2011; Fine 1985; Jayaprakash 2007; Rahmani 2006; Southern 2006; Weitz 1992). The remaining six studies that did not report any adverse effects or issues with acceptability of the chlorhexidine rinse indicated that there were no oral mucosal lesions (Charles 2004); no severe/serious adverse effects/events (Faveri 2006; Stookey 2005); or adverse reaction/effect (Feres 2012); no irreversible pathology (Lucas 1999); or pathology related to the chlorhexidine mouthrinse use (Taller 1993).

\section{Excluded studies}

We excluded 38 studies from the review (see 'Characteristics of excluded studies' table). Below is a summary of the reasons for excluding these studies.

- In seven studies it was not clear whether the studies were randomised and attempts to contact the authors for clarification were unsuccessful (Bay 1975; Goutham 2013; Lang 1982; Ousehal 2011; Paknejad 2006; Todkar 2012; Vechis-Bon 1989).

- In four studies the mechanical oral hygiene procedures were not the same in the chlorhexidine rinse and comparator arms (Bouwsma 1992; Brown 2002; Madden 2008; Subhash 1985).

- In 11 studies the comparison was with another active rinse and there was no placebo/control or mechanical oral hygiene only group (Biswas 2014; CTRI 2014; Ernst 1998; Eshwar 2016; Haffajee 2009; McKenzie 1992; NCT01750801; NCT02546804; Persson 1991; Priya 2015; Radafshar 2017).

- Four studies were excluded because the chlorhexidine arm included another active agent that the comparator arms did not have. We would have been unable to separate the effect of the chlorhexidine rinse from the effect of the other active agent (Cortelli 2015; Luoma 1978; Quirynen 2005; Quirynen 2006).

- In one study the chlorhexidine arm received a more intensive intervention including cognitive behavioural education (2 hours) compared to the control arm who received standard care (Persson 1998). In another study (Caton 1993) the chlorhexidine arm received oral hygiene instructions but the control arm did not. The mechanical oral hygiene procedures were potentially not the same in both arms in these two studies.

- One study was unpublished and we were unable to obtain a copy of the study despite following up several lines of enquiry (Segreto 1993).

- In one study, it was not clear from the published report whether the study was a randomised trial. Additional information obtained from the contact author indicated quasirandomisation (participants were assigned to groups according to the order that they came to the University) (Leyes Borrajo 2002).

- One study was reported in an abstract. The authors were contacted but they did not have access to the data (Saltini 1988).

- One study compared chlorhexidine, zinc and placebo mouthrinses but each rinse arm was further split into those who received a professional prophylaxis prior to the start of the study and those that did not. The study outcomes were not reported in a format that could be used in the review and the authors of the study were unable to provide any additional data (Fischman 1975).

- In one study (Nadkerny 2015) the duration of rinsing was only 15 days and this was not apparent until the full text of the paper was reviewed.

- In three studies we had concerns about the duplication of outcome data in different studies from the same research group (Gupta 2014; Gupta 2015; Karim 2014) and contact with the authors failed to provide an adequate explanation or reassurance. Another study from this research group was excluded due to these concerns (Gupta 2015a). 
- One study was excluded because the chlorhexidine mouthrinse was not used as an adjunct to regular mechanical oral hygiene (Yates 2002).

\section{Risk of bias in included studies}

\section{Allocation}

\section{Random sequence generation}

Nineteen studies reported sufficient details of the method of random sequence generation to allow us to assign a judgement of low risk of bias. The remaining 32 studies either stated that participants were randomised to groups, with no further details, or gave an inadequate description of the method. For these studies, we assigned a judgement of unclear risk of bias.

\section{Allocation concealment}

Eleven studies described various methods of allocation concealment which implied that the random sequence was adequately protected and administered as it was generated, thus we assigned a judgement of low risk of bias. Two studies were rated as being at high risk of bias because personnel involved in the studies used a random number table to assign participants to groups, and could have foreseen upcoming assignments (Faveri 2006; Fine 1985). The remaining 38 studies did not report sufficient information on the randomisation process to allow a judgement of either low or high risk of bias, so we assigned a judgement of unclear risk of bias to them.

Overall, seven studies had a rating of low risk of bias for the two above domains, and therefore were at low risk of selection bias. The two studies that had a rating of high risk of bias for allocation concealment were therefore at high risk of selection bias. The remaining 42 studies were at unclear risk of bias.

\section{Blinding}

The majority of studies contained a placebo arm with the intention of blinding participants and personnel. However, we considered blinding unlikely to be successful due to the propensity for chlorhexidine to stain the teeth and oral tissues and the association of its use with clinically apparent adverse effects such as mucosal desquamation and mucosal lesions.

\section{Blinding of participants and personnel (performance bias)}

Forty-one studies were assigned a judgement of high risk of bias either because staining or adverse events were reported at a higher rate in the chlorhexidine arms, or because a placebo comparison was not used and therefore no blinding was attempted. In these studies, participants could be aware of their allocated intervention and this could have affected their motivation and oral health behaviour. Two studies were assigned a judgement of low risk of bias as there was no difference in staining between the groups, so the blinding appeared to have been maintained (Ferretti 1987; Lopez-Jornet 2012). In the remaining eight studies, blinding was intended through the use of placebo but staining was not reported, so there was insufficient information on which to base a judgement. We rated these studies as being at unclear risk of bias.

\section{Blinding of outcome assessment (detection bias)}

Thirty-three studies were assigned a judgement of high risk of bias because the outcomes assessors could have noticed the higher rates of staining or adverse events or both in the chlorhexidine arms. Five studies were rated as having low risk of bias. Two of these studies showed no difference in staining between groups (Axelsson 1987; Lopez-Jornet 2012). Two studies used masking strategies (whereby a subset of participants were assessed with and without plastic tooth covers and painting of the tongue with food dyes) which revealed that measurements of gingivitis were no different when an examiner thought the participant had received chlorhexidine or not (Grossman 1986; Grossman 1989). One study performed a sensitivity analysis on participants with no visible side effects, which showed no difference to the analysis including all participants, and we judged that bias had not affected the results (Zimmer 2006). We rated the remaining 13 studies at unclear risk of bias because extrinsic tooth staining was not reported. These studies either implied that the outcome assessors were blinded, or did not discuss it, but in any case it was not possible to judge whether or not any blinding could have been successful.

\section{Incomplete outcome data}

We did not have any concerns regarding attrition bias in 23 studies, so we rated them as at low risk of bias. Nineteen studies were assigned a judgement of high risk of bias due to either a high overall proportion of attrition, differences in the proportion of attrition between groups, or because the reasons for attrition differed between groups and were linked to the intervention. The remaining nine studies were rated as unclear because the numbers per group at either baseline or evaluation were not reported, thus preventing us from calculating the attrition.

\section{Selective reporting}

Fourteen studies adequately reported all expected and planned outcomes, so we rated them as having low risk of bias. The remaining 37 studies were rated at high risk of bias, mainly due to lack of reporting of measures of variance along with the means for each group, and no reporting of tooth staining and other adverse effects.

\section{Other potential sources of bias}

Eleven studies were assigned a judgement of low risk of bias. Three studies were assigned a judgement of high risk of bias due to baseline imbalances between groups in important prognostic factors or outcomes of the review or both (Bajaj 2011; Graziani 2015; Sanz 1989). The remaining 37 studies were rated as unclear due to a lack of reporting of details of either calibration of outcome assessment or baseline measurements, meaning that we were unable to assess the potential for differential measurement of outcomes or baseline imbalances respectively.

\section{Overall risk of bias}

Fifty studies were at high risk of bias overall as they had at least one domain rated at high risk of bias. One study was rated at unclear risk of bias overall as it had at least one domain rated at unclear risk of bias, but with no domains rated at high risk of bias (LopezJornet 2012). A summary of the risk of bias of the included studies is presented in Figure 2. 
Figure 2. Risk of bias summary: review authors' judgements about each risk of bias item for each included study.

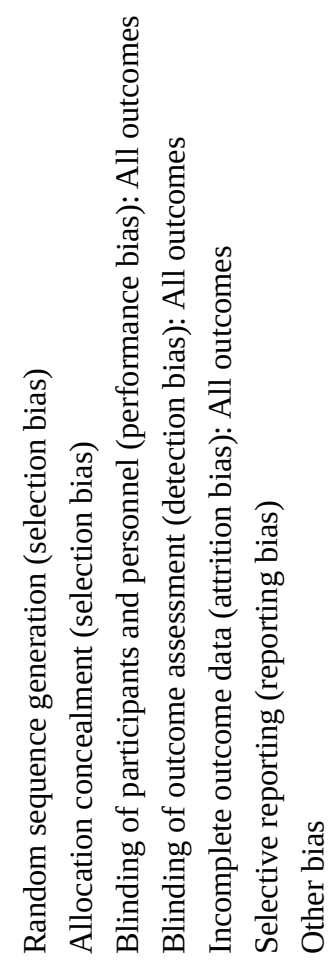

Anauate-Netto 2014

Anderson 1997

Axelsson 1987

Bajaj 2011

Banting 1989

Bhat 2014

Brecx 1993

Brightman 1991

Charles 2004

Chaves 1994

Corbet 1997

de la Rosa 1988

de la Rosa 1988b

Eaton 1997

Emling 1992

Ernst 2005

Faveri 2006

Feres 2009

Feres 2012

Ferretti 1987

Fine 1985

Flemmig 1990

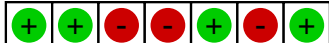

\begin{tabular}{|l|l|llllll}
\hline$?$ & $?$ & - & - & - & - & $?$ \\
\hline
\end{tabular}

\begin{tabular}{|l|l|l|llll}
\hline$?$ & $?$ & - & + & - & - & $?$ \\
\hline
\end{tabular}

\begin{tabular}{lllllll}
\hline$?$ & $?$ & - & $?$ & + & - & - \\
\hline
\end{tabular}

\begin{tabular}{|lllllll}
\hline$?$ & $?$ & - & - & - & - & $?$ \\
\hline
\end{tabular}

\begin{tabular}{lllllll}
\hline+ & $?$ & - & - & + & - & + \\
\hline
\end{tabular}

\begin{tabular}{lllllll}
\hline$?$ & $?$ & - & - & - & - & $?$
\end{tabular}

\begin{tabular}{lllllll}
\hline+ & ? & - & - & + & - & ? \\
\hline
\end{tabular}

\begin{tabular}{lllllll}
+ & $?$ & - & - & + & - & + \\
\hline
\end{tabular}

\begin{tabular}{llllllll}
\hline+ & $?$ & - & $?$ & - & - & ? \\
\hline
\end{tabular}

\begin{tabular}{lllllll}
\hline$?$ & $?$ & - & - & - & - & $?$
\end{tabular}

\begin{tabular}{|l|l|l|llll}
\hline$?$ & $?$ & - & - & $?$ & - & $?$ \\
\hline
\end{tabular}

\begin{tabular}{|l|l|llllll}
\hline$?$ & $?$ & - & - & $?$ & - & $?$ \\
\hline
\end{tabular}

\begin{tabular}{l|l|lllll}
\hline+ & $?$ & - & - & - & - & + \\
\hline
\end{tabular}

\begin{tabular}{|l|l|lllllll}
\hline$?$ & $?$ & - & - & $?$ & + & ? \\
\hline
\end{tabular}

\begin{tabular}{llllllll}
\hline$?$ & + & - & - & $?$ & + & ? \\
\hline
\end{tabular}

\begin{tabular}{|c|c|c|c|cc|c|}
\hline+ & - & $?$ & $?$ & + & - & + \\
\hline
\end{tabular}

\begin{tabular}{|c|c|c|c|c|c|c|}
\hline+ & + & $?$ & $?$ & + & - & $?$ \\
\hline
\end{tabular}

\begin{tabular}{lllllllll}
\hline$?$ & $?$ & $?$ & $?$ & + & - & $?$ \\
\hline
\end{tabular}

\begin{tabular}{llllllll}
\hline$?$ & $?$ & + & - & - & + & ? \\
\hline
\end{tabular}

\begin{tabular}{|c|ccccc|c|}
\hline+ & - & - & - & $?$ & - & $?$ \\
\hline
\end{tabular}

\begin{tabular}{lllllll}
\hline$?$ & $?$ & - & - & - & + & $?$
\end{tabular}

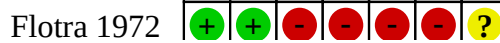


Figure 2. (Continued)

\begin{tabular}{|c|c|c|c|c|c|c|c|}
\hline Flemmig 1990 & ? & ? & & & & & ? \\
\hline Flotra 1972 & + & + & & & & & $?$ \\
\hline Graziani 2015 & + & + & & & + & & \\
\hline Grossman 1986 & $?$ & $?$ & & + & C & & $?$ \\
\hline Grossman 1989 & $?$ & $?$ & & + & ? & & $?$ \\
\hline Hase 1995 & + & $?$ & & & 1 & + & $?$ \\
\hline Hase 1998 & + & $?$ & & & 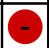 & + & $?$ \\
\hline Hoffmann 2001 & $?$ & $?$ & & & 1 & & $?$ \\
\hline Jayaprakash 2007 & $?$ & + & $?$ & $?$ & ? & & $?$ \\
\hline Jenkins 1993 & $?$ & + & 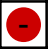 & E & + & + & $?$ \\
\hline Jose 2015 & $?$ & $?$ & & $?$ & + & & + \\
\hline yston-Bechal 1993 & $?$ & $?$ & & & & & + \\
\hline Lang 1998 & + & $?$ & & & $(-$ & + & $?$ \\
\hline Lopez-Jornet 2012 & $?$ & $?$ & + & + & + & + & $?$ \\
\hline Lucas 1999 & $?$ & $?$ & & & + & & $?$ \\
\hline Navarro 1998 & $?$ & $?$ & & 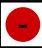 & $?$ & + & $?$ \\
\hline Overholser 1990 & + & + & & - & + & + & + \\
\hline Pereira 2011 & $?$ & $?$ & & - & + & & $?$ \\
\hline Rahmani 2006 & $?$ & $?$ & & $?$ & $?$ & & $?$ \\
\hline Sanz 1989 & + & $?$ & & - & + & & - \\
\hline Sanz 1994 & $?$ & $?$ & - & - & + & & $?$ \\
\hline Segreto 1986 & $?$ & $?$ & $?$ & $?$ & - & & $?$ \\
\hline Southern 2006 & $?$ & $?$ & $?$ & $?$ & + & & $?$ \\
\hline Stookey 2005 & $?$ & $?$ & $?$ & $?$ & & & $?$ \\
\hline Taller 1993 & $?$ & $?$ & & $?$ & + & & $?$ \\
\hline Turkoglu 2009 & $?$ & + & & & 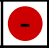 & + & + \\
\hline In Strydonck 2008 & + & $?$ & & & + & + & + \\
\hline Weitz 1992 & $?$ & $?$ & $?$ & $?$ & + & & $?$ \\
\hline Zimmer 2006 & + & + & & + & + & & + \\
\hline Zimmer 2015 & + & + & & & + & + & $?$ \\
\hline
\end{tabular}

\section{Effects of interventions}

See: Summary of findings 1 Summary of findings

\section{Chlorhexidine rinse versus placebo/control rinse or no intervention}

\section{Gingivitis}

\section{Gingival Index of Löe and Silness}

At 4 to 6 weeks, eight studies analysing 466 participants (seven at high and one at unclear risk of bias) comparing chlorhexidine rinse with placebo or control rinse and two studies analysing 339 participants (both at high risk of bias) comparing chlorhexidine with no rinse, presented data using the Gingival Index (GI) of Löe and Silness (Löe 1967; Löe and Silness 1963) (Analysis 1.1). The mean GI score across the control groups ranged from 0.17 to 1.4 with a mean of 0.93 and a median of 1 . There was no clear difference between the placebo/control and no rinse control subgroups $(\mathrm{P}=$ 0.33). The pooled effect estimate of the comparison for all trials showed a reduction in gingivitis in favour of chlorhexidine rinse (mean difference (MD) $-0.21,95 \%$ confidence interval $(\mathrm{Cl})-0.31$ to

$-0.11, \mathrm{P}<0.0001)$ demonstrating a moderate effect $(\mathrm{GI}$ on 0 to 3 increasing scale). There was substantial heterogeneity between the studies $\left(P=0.001 ;\left.\right|^{2}=67 \%\right)$.

There was no clear difference between the different control group subgroups at 6 months $(P=0.11)$. The overall effect estimate showed a reduction in gingivitis in favour of the chlorhexidine rinse (MD $-0.20,95 \% \mathrm{Cl}-0.30$ to $-0.11, \mathrm{P}<0.0001,13$ high risk of bias studies, 2616 participants) (Analysis 1.2). The mean GI score across the control groups ranged from 0.1 to 1.25 with a mean of 0.8 and a median of 0.7. There was considerable heterogeneity $(P<0.00001$; $12=96 \%)$.

There were insufficient data to determine the reduction in gingivitis associated with chlorhexidine mouthrinse use in individuals with higher mean $\mathrm{Gl}$ scores of 1.1 to 3 (moderate or severe levels of gingival inflammation).

\section{Investigation of heterogeneity}

We undertook an investigation of the reasons for heterogeneity for the $\mathrm{GI}$ scores, at both 4 to 6 weeks and 6 months, for the following 
subgroups: adults compared to children; baseline gingivitis alone compared to gingivitis and periodontitis at baseline; prophylaxis/ scale and polish at baseline compared to none; baseline mean gingivitis score $<1$ versus scores $\geq 1$, by undertaking metaregression in Stata version 14 (Stata 2015) (Additional Table 1; Table 2). The only subgroup analysis that was significant $(P=0.045)$ was for prophylaxis or not at 6 months, where the trials including a prophylaxis had a lesser effect for the chlorhexidine rinse than those without. This may explain some of the heterogeneity at 6 months.

\section{Chlorhexidine concentration and frequency of rinsing}

At 4 to 6 weeks there was no difference between the studies with concentrations of $0.2 \%$ and $0.1 \% / 0.12 \%$ chlorhexidine, and there was an insufficient number of studies in the concentration subgroups at 6 months to undertake the analysis (Additional Table 3). Participants in one study included in the meta-analysis for 4 to 6 weeks for the Gl rinsed once a day, participants in the other studies rinsing twice per day so we were unable to undertake an analysis. Participants in two studies at 6 months rinsed once per day compared with 11 rinsing twice but there was no difference between these subgroups $(P=0.56$, Additional Table 4$)$.

\section{Other data}

Meta-analyses of studies comparing chlorhexidine rinse with placebo or control rinse after 7 to 12 weeks and $>6$ months of rinsing (all at high risk of bias), using the GI of Löe and Silness (Löe 1967; Löe and Silness 1963), showed an effect size similar to the 4 to 6 weeks and 6 month studies (MD $-0.47,95 \% \mathrm{Cl}-0.76$ to -0.18 and $\mathrm{MD}-0.50,95 \% \mathrm{Cl}-1.11$ to 0.11 respectively). The uncertainty around the effect estimate at $>6$ months (two studies of 9 and 24 months duration) is reflected in the $95 \% \mathrm{Cl}$. Furthermore, there was considerable heterogeneity in both meta-analyses $\left(P<0.0001,1^{2}=\right.$ 86\%; $P<0.0001, I^{2}=99 \%$ respectively) (Additional Table 5).

One 4-week study (Anauate-Netto 2014) at high risk of bias that was not included in the meta-analysis found no statistically significant differences in Papillary Bleeding Score (Loesche 1979) between the chlorhexidine (0.12\%) and placebo group. Three studies (Fine 1985; Hoffmann 2001; Overholser 1990) at high risk of bias, reporting gingival inflammation at 4 to 6 months were not included in the meta-analysis. Two of the three studies found a reduction in the $\mathrm{GI}(\mathrm{P}=0.043)$ (Hoffmann 2001) and the modified $\mathrm{GI}(\mathrm{P}<0.001)$ (Overholser 1990) for the $0.1 \%$ and $0.12 \%$ chlorhexidine groups respectively compared to the control rinse (Additional Table 6). Results for 7 to 12 weeks studies not included in meta-analyses are supportive of a reduction in gingival inflammation associated with chlorhexidine mouthrinse use (Additional Table 6).

\section{Gingival bleeding}

A variety of different indices were used to measure gingival bleeding, therefore standardised mean differences (SMD) are presented. There was no clear difference between the placebo/ control and the no rinse subgroups at either 4 to 6 weeks or 6 months (Analysis 1.3; Analysis 1.4). At 4 to 6 weeks, eight studies analysing 649 participants (all at high risk of bias) comparing chlorhexidine rinse with placebo/control or with no rinse that reported gingival bleeding were combined in a meta-analysis. The overall effect estimate for all studies, both placebo/control and no rinse controls, was moderate at 4 to 6 weeks (SMD $-0.56,95 \% \mathrm{Cl}$ -0.79 to -0.33 ) showing a reduction in gingival bleeding in favour of the chlorhexidine rinse $(P<0.00001)$. There was evidence of moderate heterogeneity $\left(P=0.16 ; 1^{2}=34 \%\right)$.

At 6 months, eight studies analysing 1132 participants (all at high risk of bias) reported gingival bleeding and were combined in a meta-analysis. The overall effect estimate for all studies was also moderate at 6 months (SMD $-0.72,95 \% \mathrm{Cl}-1.02$ to -0.42 ), showing a reduction in gingival bleeding in favour of the chlorhexidine rinse $(P<0.00001)$. There was evidence of considerable heterogeneity between the studies at 6 months $\left(P<0.00001 ;\left.\right|^{2}=81 \%\right)$.

\section{Chlorhexidine concentration and frequency of rinsing}

No statistically significant differences were found between the subgroups with different chlorhexidine concentrations, at 4 to 6 weeks and 6 months (Additional Table 3). At both 4 to 6 weeks and 6 months all participants in the included studies for gingival bleeding were instructed to rinse twice per day, so no analysis for the effect of frequency of rinsing could be undertaken (Additional Table 4).

\section{Other data}

A meta-analysis of two studies (both at high risk of bias) analysing 196 participants comparing chlorhexidine rinse with no rinse controls using the Papillary Bleeding Index (Muhlemann 1977; Saxer 1975) after 8 weeks of rinsing showed no evidence of a difference in gingival bleeding (MD $-0.07,95 \% \mathrm{Cl}-0.16$ to 0.02 ) (Additional Table 5).

Another meta-analysis of five studies (all at high risk of bias) analysing 182 participants comparing chlorhexidine rinse with placebo rinse using a variety of different gingival bleeding indices after 7 to 12 weeks of rinsing showed a strong effect in favour of the chlorhexidine rinse (SMD $-1.29,95 \% \mathrm{Cl}-1.85$ to $-0.72, \mathrm{P}<0.00001$ ). There was evidence of substantial heterogeneity $\left(P=0.02 ; 1^{2}=64 \%\right)$ (Additional Table 5).

Three studies analysing 99 participants (all at high risk of bias) comparing chlorhexidine rinse with placebo rinse, using bleeding on probing, presented longer-term data 1, 4 and 10 months after chlorhexidine mouthrinsing had ceased and were combined in a meta-analysis that showed a small retained effect in favour of chlorhexidine (MD - $0.12,95 \% \mathrm{Cl}-0.20$ to -0.04) (Additional Table 5).

Three studies (Axelsson 1987; Graziani 2015; Sanz 1989) at high risk of bias reporting gingival bleeding at 4 to 6 weeks were not included in the meta-analysis (Additional Table 7). All three studies reported a reduction in gingival bleeding for $0.1 \%, 0.12 \%$ and $0.2 \%$ chlorhexidine rinses compared to placebo or control rinse.

Four studies (Banting 1989; Charles 2004; Lucas 1999; Sanz 1994) at high risk of bias reporting gingival bleeding at 6 months were not included in the meta-analysis (Additional Table 7). Three of these studies reported a reduction in gingival bleeding for $0.12 \%$ chlorhexidine rinse compared to placebo or control rinse. Banting 1989 demonstrated a reduction in gingival bleeding at 2 years for $0.12 \%$ chlorhexidine compared to placebo $(P<0.0001)$. Results for 7 to 12 weeks studies not included in meta-analyses are supportive of a reduction in gingival bleeding associated with chlorhexidine mouthrinse use (Additional Table 7).

\section{Plaque}

A variety of different indices were used to measure plaque, therefore SMDs are presented. There was no difference between the 
placebo/control or no rinse control subgroups for plaque at 4 to 6 weeks $(P=0.93$ ) (Analysis 1.5). The overall effect was based on 12 trials analysing 950 participants, 11 at high and one at unclear risk of bias, and showed a large effect in favour of chlorhexidine rinse (SMD $-1.45,95 \% \mathrm{Cl}-1.90$ to -1.00$)$. Four of these trials analysing 223 participants reported the Plaque Index ( 0 to 3 increasing scale) (Silness and Löe 1964) and showed a reduction in plaque in favour of chlorhexidine rinse (MD $-0.58,95 \% \mathrm{Cl}-0.78$ to -0.39) (Analysis 1.6); five of these trials analysing 546 participants reported the Turesky modification of the Quigley Hein Index (0 to 5 increasing scale) (Turesky 1970) and also showed a reduction in plaque in favour of chlorhexidine rinse (MD - $0.78,95 \% \mathrm{Cl}-0.85$ to -0.70 ) (Analysis 1.7 ).

At 6 months, although both subgroups showed a reduction in plaque favouring the chlorhexidine rinse (Analysis 1.8), there was a difference between the size of the effect estimates for the placebo/ control and no rinse subgroups $(P=0.02)$. The higher estimate for the chlorhexidine rinse compared to placebo/control rinse based on nine trials analysing 1933 participants (all at high risk of bias) with a large effect (SMD $-1.59,95 \% \mathrm{Cl}-1.89$ to -1.29 ) is compared with a moderate effect for the chlorhexidine rinse compared to no rinse control groups based on 2 trials analysing 142 participants (both at high risk of bias) (SMD $-0.68,95 \% \mathrm{Cl}-1.35$ to -0.01 ). There was substantial heterogeneity among the studies in each subgroup $\left(P<0.00001 ; I^{2}=84 \% ; P=0.06 ; 1^{2}=72 \%\right.$ for placebo/control and no rinse subgroups respectively). Five studies analysing 1108 participants reported data for the Plaque Index at 6 months. There was no clear difference between the placebo/control and no rinse subgroups, and the overall effect was $-0.62(95 \% \mathrm{Cl}-1.12$ to -0.12$)$ (Analysis 1.9). Six trials, all with placebo/no treatment controls, analysing 967 participants reported the Turesky modification of the Quigley Hein Index at 6 months and also showed a reduction in plaque in favour of chlorhexidine rinse (MD $-0.73,95 \% \mathrm{Cl}-0.88$ to -0.57) (Analysis 1.10).

\section{Chlorhexidine concentration and frequency of rinsing}

No differences were found between the subgroups with different chlorhexidine concentrations (Additional Table 3). At 4 to 6 weeks the participants in one trial were instructed to rinse once per day compared with twice a day in the other 11 trials so no analysis for the effect of frequency of rinsing could be undertaken. Similarly at 6 months the participants in one trial were instructed to rinse once per day compared with twice a day in the other 10 trials so no analysis for the effect of frequency of rinsing could be undertaken (Additional Table 4).

\section{Other data}

Meta-analyses of studies that compared chlorhexidine mouthrinse with placebo (10 studies all at high risk of bias analysing 423 participants) or no rinse control (two studies both at high risk of bias analysing 196 participants) at 7 to 12 weeks showed a reduction in plaque (using various indices) in favour of chlorhexidine rinse (SMD $-1.74,95 \% \mathrm{Cl}-2.51$ to -0.98 and SMD $-0.77,95 \% \mathrm{Cl}-1.07$ to -0.47 respectively, Additional Table 5) both indicating a strong effect. There was considerable heterogeneity among the 10 placebocontrolled studies $\mathrm{P}<0.00001 ; \mathrm{I}^{2}=91 \%$.

One large 9-month study (at high risk of bias) (Bajaj 2011) analysing 852 participants comparing $0.1 \%$ chlorhexidine rinse with distilled water found a large reduction in plaque ( 0 to 3 increasing scale) in the chlorhexidine rinse group (MD $-1.55,95 \% \mathrm{Cl}-1.79$ to -1.31 , Additional Table 5).
Four studies analysing 132 participants (all at high risk of bias) comparing chlorhexidine rinse with placebo rinse presented data 1 , 4 and 10 months after rinsing had ceased, using a variety of indices, and were combined in a meta-analysis that showed a large retained effect in favour of chlorhexidine (SMD $-1.10,95 \% \mathrm{Cl}-1.18$ to -0.40 , Additional Table 5). However, there was substantial heterogeneity between the studies $\left(P=0.02 ; 1^{2}=71 \%\right)$.

One study (Sanz 1989) at high risk of bias reporting a plaque outcome at 6 weeks that was not included in the meta-analysis showed a reduction in plaque for $0.12 \%$ chlorhexidine rinse compared to placebo rinse (Additional Table 8).

Five studies at high risk of bias reporting a plaque outcome at 6 months that were not included in the meta-analysis also showed a reduction in plaque for $0.12 \%, 0.1 \%, 0.05 \%$ and $0.06 \%$ chlorhexidine rinse compared to placebo or control rinse (Banting 1989; Hoffmann 2001; Jayaprakash 2007; Lucas 1999; Sanz 1994) (Additional Table 8).

Banting 1989, which was not included in the meta-analysis demonstrated a reduction in plaque at 2 years for $0.12 \%$ chlorhexidine compared to placebo. Results for 7 to 12 weeks studies not included in meta-analyses were generally supportive of a reduction in plaque associated with chlorhexidine mouthrinse use (Additional Table 8).

\section{Adverse effects}

\section{Calculus}

Two studies analysing 102 participants, both at high risk of bias, and with placebo/control rinses, presented data on calculus at 4 to 6 weeks. There was insufficient evidence to determine whether using chlorhexidine rinse for 4 to 6 weeks was associated with increased calculus formation (SMD $0.02,95 \% \mathrm{Cl}-0.09$ to 0.14 ) (Analysis 1.11).

At 7 to 12 weeks, five studies (all high risk of bias) analysing 330 participants, with a placebo/control rinse presented calculus data, along with one study analysing 95 participants with a no rinse control also at high risk of bias (Analysis 1.12). There was a difference between the subgroups for control type, with there being insufficient evidence to determine an effect on calculus for chlorhexidine rinse compared to placebo/control rinse (SMD 0.14, $95 \% \mathrm{Cl}-0.08$ to 0.36 ). The single study in the no rinse control group, however, showed more calculus in the chlorhexidine rinse group compared to the no rinse control group (SMD 1.02, 95\% Cl 0.59 to 1.45$)$ demonstrating a large effect. There was evidence of substantial heterogeneity $\left(P=0.004 ; 1^{2}=71 \%\right)$.

At 6 months there was a difference between the subgroups for control type. There was only one study (at high risk of bias), analysing 91 participants, with a no rinse control group which showed an increase in calculus in the chlorhexidine rinse group (SMD 1.39, 95\% Cl 0.93 to 1.85 ) demonstrating a large effect (Analysis 1.13). The pooled result for the three studies (at high risk of bias) analysing 232 participants with placebo/control rinses showed an increase in calculus associated with chlorhexidine rinse (SMD $0.60,95 \% \mathrm{Cl} 0.24$ to 0.96 ) demonstrating a moderate effect. There was evidence of moderate heterogeneity $\left(P=0.16 ;\left.\right|^{2}=46 \%\right)$.

\section{Chlorhexidine concentration and frequency of rinsing}

No statistically significant difference was found between the subgroups with different chlorhexidine concentrations at 7 to 12 
weeks, however the data at 6 months indicated a larger effect (increased calculus formation in the chlorhexidine rinse group) for the lower concentration chlorhexidine mouthrinse (Additional Table 3). Participants in all the included trials with calculus data at 4 to 6,7 to 12 weeks and 6 months were asked to use the mouthrinse twice a day, so no analysis of frequency could be undertaken (Additional Table 4).

\section{Other data}

Five studies at high risk of bias reporting interim and end scores for calculus at various time points from 4 weeks to 2 years (Anderson 1997; Banting 1989; Charles 2004; Grossman 1986; Sanz 1994) that were not included in the meta-analysis all found increased supragingival calculus in the $0.12 \%$ chlorhexidine rinse groups compared to the placebo and control rinse groups (Additional Table 9). Increased calculus was noted in two other studies but not measured using an index or scale (de la Rosa 1988; de la Rosa 1988b).

\section{Extrinsic tooth staining}

Different dichotomous and continuous indices were used to measure tooth staining. At 4 to 6 weeks both the dichotomous data (two studies at high risk of bias, 156 participants) and the continuous data (eight studies at high risk of bias, 415 participants) showed more tooth staining in the chlorhexidine rinse group. A large effect estimate was found for both the dichotomous data (risk ratio (RR) $5.41,95 \% \mathrm{Cl} 2.03$ to 14.47 , Analysis 1.14) and continuous data (SMD 1.07, 95\% Cl 0.80 to 1.34 , Analysis 1.16). There was no strong evidence of heterogeneity between the trials reporting continuous data $\left(P=0.14,1^{2}=36 \%\right)$.

Only one study, analysing 118 participants, reported dichotomous data for tooth staining at 7 to 12 weeks, with more tooth staining in the chlorhexidine group (RR $2.50,95 \% \mathrm{Cl} 1.29$ to 4.83) (Analysis 1.15). Eleven studies, analysing 581 participants (at high risk of bias) reported data on different continuous scales showing an increase in tooth staining associated with chlorhexidine rinse at 7 to 12 weeks (SMD 1.19, 95\% Cl 0.98 to 1.40), demonstrating a large effect (Analysis 1.17).

Four studies analysing 323 participants, at high risk of bias, reported continuous data at 6 months using different tooth staining indices. The pooled effect estimate indicated a large effect for tooth staining due to the chlorhexidine rinse (SMD 1.54, 95\% Cl 1.22 to 1.86) (Analysis 1.18). There was no strong evidence of heterogeneity $\left(P=0.19 ;\left.\right|^{2}=36 \%\right)$.

\section{Chlorhexidine concentration and frequency of rinsing}

Subgroup comparisons for chlorhexidine concentration were not significant (Additional Table 3). In one study providing tooth staining data at both 4 to 6 weeks and 7 to 12 weeks, participants rinsed three times a day, and in one of the studies at 4 to 6 weeks participants rinsed only once per day, whereas in all the other studies participants rinsed twice per day (Additional Table 4). There were insufficient studies in the subgroups to make a meaningful comparison on frequency at any time point.

\section{Other data}

Thirteen studies reported information about tooth staining that could not be included in meta-analyses. Eight studies that reported data on extrinsic tooth staining could not be included in meta- analyses because no measure of variance was reported (Charles 2004; Flotra 1972; Grossman 1989; Hoffmann 2001; Sanz 1994), no outcome data were reported (Grossman 1986) or because the mean value in the control groups was zero (Axelsson 1987; Graziani 2015). All of these studies reported higher levels of extrinsic tooth staining in the chlorhexidine compared to control arms at 4 to 6 weeks (Axelsson 1987; Flotra 1972; Graziani 2015); 7 to 12 weeks (Charles 2004; Grossman 1989); and at 6 months (Charles 2004; Grossman 1986; Grossman 1989; Hoffmann 2001; Sanz 1994). In five other studies tooth staining was reported combined with either denture staining (Lopez-Jornet 2012), discolouration of oral tissues (Hase 1995; Turkoglu 2009; Zimmer 2006) or was reported as 'discolouration' and it was not clear whether this related to teeth, oral tissues or both (Bhat 2014). In four of these five studies staining/discolouration was higher in the chlorhexidine rinse groups at 4 to 6 weeks (Bhat 2014; Hase 1995; Turkoglu 2009; Zimmer 2006) and 7 to 12 weeks (Zimmer 2006) (Additional Table 10).

In five additional studies, increased tooth staining (de la Rosa 1988; de la Rosa 1988b; Feres 2009), 'pigmentation' (Pereira 2011) and 'yellow teeth' (Anauate-Netto 2014) were reported in relation to the chlorhexidine rinse arms. In two studies staining of teeth in the chlorhexidine rinse arm was listed as a reason for participant withdrawal from the studies (Banting 1989; Eaton 1997).

\section{Other adverse effects}

Twenty-two studies reported at least one adverse effect apart from extrinsic tooth staining and calculus formation in the chlorhexidine rinse arms. Apart from extrinsic tooth staining, the adverse effects most commonly reported in the chlorhexidine rinse arms of the included studies were taste disturbance/alteration (reported in 11 studies), effects on the oral mucosa (reported in 13 studies) and a general burning sensation or a burning tongue or both (reported in nine studies).

Taste disturbance/alteration was reported in 11 studies (AnauateNetto 2014; Bhat 2014; Ernst 2005; Graziani 2015; Hase 1998; Jenkins 1993; Jose 2015; Lang 1998; Pereira 2011; Turkoglu 2009; Zimmer 2006). The proportion of participants experiencing taste disturbance/alteration was reported in eight of these studies and was consistently higher in the chlorhexidine rinse arms (3\% to $30 \%)$ than in the placebo/control/oral hygiene $(\mathrm{OH})$ only arms $(0 \%$ to $7 \%)$ with one exception (Bhat 2014) where a higher proportion of participants in the saline control arm experienced taste disturbances (45.4\% versus $63.6 \%$ ).

A general burning sensation (Anderson 1997; Bhat 2014; Emling 1992; Hase 1995; Sanz 1989; Zimmer 2006) and/or a burning tongue (Anauate-Netto 2014; Jose 2015; Lang 1998) was reported in the chlorhexidine rinse arms in nine studies. The proportion of participants affected was reported in four of the nine studies (Anderson 1997; Bhat 2014; Jose 2015; Zimmer 2006) and ranged from $4.6 \%$ to $50 \%$ in the chlorhexidine arms compared to $0 \%$ in the placebo/control/OH only arms. Hase 1995 reported 'burning sensation' rated by participants on a visual analogue scale from none (0) to 100 (very much). The mean visual analogue scale score for the chlorhexidine rinse arm was approximately 18 compared to 8 for the placebo arm.

Effects on the oral mucosa of participants in the chlorhexidine rinse arms were reported in 13 studies. Specific effects were described as 
mucosal irritation (Anauate-Netto 2014; Emling 1992), sensitivities of the oral mucosa (Ernst 2005), mucosal soreness (Jenkins 1993), mild desquamation (de la Rosa 1988; de la Rosa 1988b; Navarro 1998), mucosal ulceration (Hase 1995; Turkoglu 2009), mucosal erosions (Jenkins 1993), soft tissue conditions (Banting 1989), oral mucosal lesions (Axelsson 1987; Flotra 1972) and adverse mucosal reaction (Eaton 1997). Where it was reported, the proportion of participants experiencing effects on the oral mucosa was generally low. For example, clinically apparent mucosal ulceration was reported in $12 \%(n=3)$ of participants in the chlorhexidine rinse arm of one study (Turkoglu 2009) and mucosal erosions affected $4 \%$ (n $=2$ ) of participants in the chlorhexidine rinse arm of another study (Jenkins 1993). However one study reported a high proportion of oral mucosal lesions in participants in the chlorhexidine $(0.2 \%)$ rinse $\operatorname{arm}(25 \%, n=6)$ (Axelsson 1987).

Discolouration/pigmentation of the oral soft tissues was reported in seven studies and was consistently higher in the chlorhexidine rinse arms ranging from $9 \%$ to $88 \%$ compared to $0 \%$ to $7 \%$ in the placebo/control/OH only arms (Graziani 2015; Pereira 2011; Hase 1995; Hase 1998; Lang 1998; Turkoglu 2009; Zimmer 2006). Coated tongue was reported as an adverse effect in one study (Jose 2015).

Less commonly reported adverse effects included transient anaesthetic sensation, hypoaesthesia and paraesthesia; affecting $5 \%$ to $18 \%$ of participants in the chlorhexidine rinse arms (Hase 1995; Hase 1998; Jenkins 1993; Jose 2015; Lang 1998; Zimmer 2006).

Lastly, in relation to acceptability, an unacceptable or unpleasant taste was reported by participants in the chlorhexidine rinse arms of eight studies (Anauate-Netto 2014; Chaves 1994; Eaton 1997; Feres 2009; Hase 1995; Jenkins 1993; Sanz 1989; Segreto 1986).

\section{Sensitivity analysis}

We undertook a sensitivity analysis for the $\mathrm{Gl}$ at both 4 to 6 weeks and 6 months, excluding trials where we had to estimate the standard deviation from similar studies, or had to read the data from graphs. The pooled estimate for 4 to 6 weeks (excluding Jenkins 1993 and Sanz 1989) was: MD $-0.23(95 \% \mathrm{Cl}-0.36$ to -0.10), and at 6 months (excluding Banting 1989; Chaves 1994; Jayaprakash 2007; Lucas 1999; Sanz 1994) MD - 0.25 (95\% Cl -0.39 to $-0.10)$. These estimates are similar to those for the overall pooling of the studies.

We undertook a sensitivity analysis for the $\mathrm{Gl}$ at both 4 to 6 weeks and 6 months excluding trials where professional periodontal treatment was carried out during the study (i.e. Faveri 2006; Feres 2009; Feres 2012; Flotra 1972; Rahmani 2006; Sanz 1989). The pooled estimate for 4 to 6 weeks (excluding Rahmani 2006 and Sanz 1989 ) was: MD $-0.21(95 \% \mathrm{Cl}-0.32$ to -0.10$)$, and at 6 months (excluding Flotra 1972) MD $-0.19(95 \% \mathrm{Cl}-0.29$ to -0.10$)$. These estimates are similar to those for the overall pooling of the studies.

\section{Assessment of reporting biases}

The meta-analysis for $\mathrm{Gl}$ at 4 to 6 weeks included 10 trials. Testing for asymmetry in a funnel plot using the methods described by Egger 1997, using "metabias" command in Stata $(P=0.019)$, although significant, the estimate of bias was negative indicating that the bias was lack of large studies (not small studies). The meta-analysis for $\mathrm{Gl}$ at 6 months included 13 trials, and there was no evidence of publication bias from the funnel plot and this was confirmed by testing for asymmetry in a funnel plot using the same methods $(P=0.95)$. Two of the plaque meta-analyses at 4 to 6 weeks and 6 months had more than 10 studies but funnel plots cannot be investigated when SMD models are used. All the other meta-analyses had less than 10 trials, so we were unable to assess reporting bias for these.

\section{DISCUSSION}

\section{Summary of main results}

There was high-quality evidence that using chlorhexidine mouthrinse as an adjunct to regular mechanical oral hygiene procedures for 4 to 6 weeks and 6 months results in a moderate reduction in gingivitis compared to placebo, control or mechanical oral hygiene alone. This reduction in gingivitis, in individuals with mild gingival inflammation on average (mean score of 1 on the 0 to 3 Gingival Index (GI) scale) is not considered to be clinically important. Heterogeneity was considerable in the meta-analyses at 4 to 6 weeks and 6 months and subgroup analysis was only able to explain some of the heterogeneity at 6 months, due to whether or not a prophylaxis or scale and polish was conducted at the start of the study. The standardised mean differences (SMDs) for gingival bleeding at 4 to 6 weeks and 6 months also indicate that chlorhexidine mouthrinse has a moderate effect on gingival bleeding compared to placebo, control or mechanical oral hygiene alone. There was insufficient evidence to determine the reduction in gingivitis associated with chlorhexidine mouthrinse use in individuals with mean GI scores of 1.1 to 3 indicating moderate or severe levels of gingival inflammation.

There was also high-quality evidence that chlorhexidine mouthrinse reduces plaque at 4 to 6 weeks and 6 months. SMDs for plaque for both control subgroups at 4 to 6 weeks and at 6 months indicate a large effect. The four studies that used the Plaque Index ( 0 to 3 increasing scale) and the four studies that used the Turesky Modification of the Quigley and Hein Index ( 0 to 5 increasing scale) at 4 to 6 weeks indicate a reduction in plaque in favour of the chlorhexidine mouthrinse.

There was no evidence that one concentration of chlorhexidine rinse was more effective than another in reducing gingivitis and plaque. Participants in most of the studies included in the metaanalyses used a rinse frequency of twice daily, limiting our ability to analyse the effect of rinse frequency on gingivitis and plaque. We were only able to evaluate the effect of rinse frequency for gingivitis measured at 6 months using the $\mathrm{GI}$, however, no difference in effect was observed.

There was moderate-quality evidence that chlorhexidine rinse causes a large amount of extrinsic tooth staining when used for periods of 4 weeks or longer. Comparison of subgroups with different chlorhexidine concentrations was not statistically significant.

There was insufficient evidence to determine whether using chlorhexidine rinse for 1 to 3 months was associated with increased calculus formation. There was some evidence of increased calculus formation related to rinsing with chlorhexidine mouthrinse for a period of 6 months. However, overall evidence for the effect of chlorhexidine mouthrinse on calculus formation was inconclusive. 
Twenty-two of the 51 studies included in this review reported at least one adverse effect apart from extrinsic tooth staining and calculus formation in the chlorhexidine rinse arms. The adverse effects most commonly reported in the chlorhexidine rinse arms of the included studies were taste disturbance/alteration (reported in 11 studies), effects on the oral mucosa including mucosal irritation, soreness, mild desquamation, mucosal ulceration/erosions and oral mucosal lesions (reported in 13 studies) and a general burning sensation or a burning tongue or both (reported in nine studies).

\section{Overall completeness and applicability of evidence}

Chlorhexidine mouthrinse is effective in reducing gingivitis and plaque when used as an adjunct to regular mechanical oral hygiene procedures for 4 weeks or longer. The consistency of effect for chlorhexidine mouthrinse on gingivitis and plaque across studies included in this review was remarkable though not surprising considering that chlorhexidine mouthrinse has long been regarded as the gold standard of antimicrobial mouthrinses.The primary outcome of this review was gingivitis which can be measured using a variety of different indices. The clinical features of gingivitis can be assessed non-invasively by visual assessment of colour, contour and gingival bleeding or invasively using an instrument to provoke bleeding or both (Lorenz 2009). Our review clearly demonstrates the many different indices that can be used to measure gingivitis. While these many indices were developed to provide a standardised reproducible method of quantifying gingivitis for descriptive or comparative purposes in epidemiology and clinical trials respectively, the subjectivity involved in their use remains problematic. The presence/absence of bleeding on provocation would appear to be the more objective approach (Ciancio 1986; Lorenz 2009; Polson and Caton 1985). However, the probing technique, the depth to which the instrument is inserted, the force with which it is applied, the time allowed to elapse between applying the stimulus and recording the outcome and even the angulation of the instrument can have an effect on the bleeding response (Lorenz 2009; Polson and Caton 1985; van der Weijden 1994). Moreover, calibration of examiners and demonstrating inter- and intraexaminer reliability are hampered by the inability to obtain the same response upon repeated application of the stimulus to provoke gingival bleeding at the same site (Lorenz 2009; Polson and Caton 1985). In synthesising the evidence from primary research studies evaluating chlorhexidine mouthrinse as an adjunctive treatment for gingival health, the many different approaches to measuring gingivitis, particularly gingival bleeding, presented difficulties in combining the study results to produce a summary of the effect of the mouthrinse.

To facilitate the analysis, we grouped the indices into those that measured principally gingival inflammation visually and invasively (e.g. the Gingival Index (Löe 1967; Löe and Silness 1963)) or visually only (e.g. the Modified Gingival Index (Lobene 1986)) and those that measured gingival bleeding (e.g. bleeding on probing (BOP) (Ainamo and Bay 1975) or the Eastman Interdental Bleeding Index (Caton and Polson 1985)). We endeavoured to include both types of index for measuring gingivitis in the review, therefore where both were reported within a study, both were data extracted. Our main analysis was based on the studies that reported gingival inflammation using the Gingival Index (Löe 1967; Löe and Silness 1963) at 4 to 6 weeks and 6 months to reflect both the short- and long-term effect of chlorhexidine mouthrinse. Our results showed that after 4 to 6 weeks of use, chlorhexidine mouthrinse reduced gingivitis by a mean of 0.21 of a unit on the Gingival Index (GI) scale when compared to a placebo/control mouthrinse or mechanical oral hygiene alone. Based on these studies, a reduction in gingivitis of 0.11 to 0.31 of a unit on the $\mathrm{Gl}$ scale could be expected with use of chlorhexidine mouthrinse for a period of 4 to 6 weeks in individuals with mild gingival inflammation on average (mean score of 1 on the 0 to $3 \mathrm{Gl}$ scale). A similar mean effect size of 0.20 of a unit reduction on the $\mathrm{Gl}$ scale was found for gingivitis at 6 months also in individuals with mild gingival inflammation on average.

The $\mathrm{Gl}$ is measured on a 0 to 3 increasing scale with 0 indicating normal gingivae. However, each one unit increase on the scale does not indicate the same proportionate deterioration in gingival condition and a low mean $\mathrm{Gl}$ score could mask the presence of a small number of sites with moderate or severe levels of gingival inflammation. With this in mind, depending on the average gingivitis level in an individual, a one unit reduction could indicate a shift from severe inflammation and a tendency for spontaneous bleeding to moderate inflammation and BOP or from moderate inflammation and BOP to mild inflammation but no BOP. As previously mentioned, the participants who contributed data to the meta-analyses of the $\mathrm{Gl}$ at 4 to 6 weeks had a low mean gingival index score at baseline. The clinical relevance of a mean reduction of one fifth of a unit on the GI scale is therefore not clear. Our results for gingival bleeding indicate that using chlorhexidine mouthrinse for 4 to 6 weeks or 6 months has a moderate effect on gingival bleeding. Although these meta-analyses included some studies where participants appeared to have a high proportion of sites with BOP at baseline, the variety of different gingival bleeding indices employed meant that we were unable to determine the mean reduction in gingivitis associated with chlorhexidine mouthrinse use in individuals with moderate or severe levels of gingival inflammation. When the SMDs for gingival bleeding were expressed in the units of the bleeding aspect of the $\mathrm{GI}(\mathrm{Gl} \geq 2)$ the effect size was equivalent to a $7 \%$ reduction in the percentage of sites with $B O P$ at 4 to 6 weeks and a $4 \%$ reduction in the percentage of sites with BOP at 6 months. Use of chlorhexidine mouthrinse was associated with a large reduction in plaque at 4 to 6 weeks and 6 months. The mean reduction in plaque associated with chlorhexidine mouthrinse use at 4 to 6 weeks was equivalent to approximately half a unit on the 0 to 3 Plaque Index scale (Silness and Löe 1964) or three quarters of a unit on the 0 to 5 Turesky modification of the Quigley and Hein Index (TQH) scale (Turesky 1970).

Statistical heterogeneity was evident in many of our metaanalyses and was likely due to variation in the characteristics of the individual included studies. Investigation of the observed heterogeneity was conducted for our main outcome, measured using the $\mathrm{Gl}$ at 4 to 6 weeks and 6 months using prespecified characteristics. There was a decrease in the effect estimate for the $\mathrm{Gl}$ at 6 months when a prophylaxis or scale and polish was conducted at the start of the study compared to studies where no prophylaxis or scale and polish was undertaken. This is not consistent with our knowledge of the mechanism of action of chlorhexidine mouthrinse which would appear to be more effective in preventing plaque accumulation when applied to a clean tooth surface than reducing pre-existing plaque (Eley 1999). Nonetheless, the results indicate that some of the heterogeneity for the $\mathrm{GI}$ at 6 months was due to whether or not a prophylaxis or scale and polish was conducted at the beginning of the study. We have presented all of the meta-analyses despite the high heterogeneity due to 
the remarkable consistency of the direction of effect across the different analyses.

We included studies that evaluated chlorhexidine mouthrinse use in individuals with gingivitis only or gingivitis and periodontitis. Where it was reported, the level of gingivitis in the included studies at baseline was generally low. The majority of the studies that evaluated chlorhexidine mouthrinse use in individuals with periodontitis evaluated its effect as an adjunct to surgical or nonsurgical periodontal treatment. In a small number of the studies that evaluated chlorhexidine mouthrinse use in individuals with periodontitis, it was difficult to ascertain whether the professional tooth cleaning provided was appropriate for the level of disease present. For example, in two of these studies it was reported that professional cleaning was undertaken at baseline but no further details were provided (Chaves 1994; Ernst 2005). In another study where $17 \%$ to $50 \%$ of participants in the study groups were diagnosed with periodontitis, supragingival scaling and polishing only was provided at baseline (Graziani 2015). In one study a proportion of the participants clearly had periodontitis and abundant calculus, however no professional treatment or oral hygiene instructions were provided to the participants (Corbet 1997). The inclusion of these studies could be criticised because antimicrobials such as chlorhexidine have limited capacity to penetrate subgingivally and are therefore suggested for use as an adjunct to mechanical debridement rather than as an alternative therapy (Greenstein 1986; Ohrn 2009). Furthermore, self-medication with an effective antimicrobial agent such as chlorhexidine mouthrinse "may mask more serious underlying periodontal disease" (Tonetti 2015). It is well recognised that control of supragingival plaque alone will not be sufficient to treat periodontitis (Eley 1999; Hull 1980), disruption of the subgingival plaque by mechanical debridement will also be required (Kornman 1986; Ohrn 2009).

We would have liked to investigate how the effect of chlorhexidine mouthrinse on gingival health varies depending on the level of underlying periodontal disease. However, the periodontal status of the participants in the included studies often was not clearly reported at baseline. In many cases, the inclusion criteria allowed for individuals to have pocket probing depths and clinical attachment loss consistent with periodontitis but the periodontal status of the people actually enrolled in the study was not described. Furthermore, where participants were advised to continue their regular oral hygiene practices during the study, no information about their frequency of toothbrushing or their use of interdental cleaning aids was reported. Therefore, our ability to explore the effect of the adjunctive use of chlorhexidine mouthrinse on gingivitis in individuals with different levels of periodontal disease at baseline and in individuals with treated and untreated periodontitis was hampered both by the small number of studies available at each time point and by poor reporting of the relevant information in the included studies.

We were able to conduct a metaregression analysis of 4 to 6 week and 6-month studies where participants had gingivitis only compared to studies where participants had gingivitis and periodontitis (both treated and untreated during the study). The results did not disclose any statistically significant difference in the effect of the chlorhexidine mouthrinse on the gingival index at either time point, however only eight studies were included in the metaregression at 4 to 6 weeks and only nine studies were included in the metaregression at 6 months. We cannot specifically comment on the effect of chlorhexidine used adjunctively during periodontal treatment on periodontal outcomes such as pocket probing depth and clinical attachment loss because the focus of this review was gingivitis and therefore periodontal outcomes were outside the scope of the review.

Retention of chlorhexidine in the oral cavity is key to its effectiveness as an antimicrobial agent (Bonesvoll 1974; Bonesvoll 1974a; Bonesvoll 1978; Jenkins 1988; Rolla 1971) and is dependant on a number of different factors including concentration and duration of application (Tomás 2010). In relation to concentration, the effect of chlorhexidine on plaque is dose-dependant and a similar effect can be achieved with larger volumes of lower concentration solutions providing the optimal dose of approximately $20 \mathrm{mg}$ twice a day is delivered (Bonesvoll 1978). All but four of the included studies evaluated either $0.2 \%, 0.12 \%$ or $0.1 \%$ chlorhexidine mouthrinse and only two of these studies provided data for $0.2 \%$ compared to $0.1 \%$ (Axelsson 1987) or $0.12 \%$ (Segreto 1986) chlorhexidine mouthrinse within the same study. Most of the studies that evaluated $0.2 \%$ chlorhexidine mouthrinse employed a rinse volume of $10 \mathrm{~mL}$ twice a day while most of the studies that evaluated $0.12 \%$ or $0.1 \%$ chlorhexidine mouthrinse employed a rinse volume of $15 \mathrm{~mL}$ twice a day. Therefore regardless of the concentration of chlorhexidine mouthrinse used, the dose of chlorhexidine was effectively the same in the majority of the studies and corresponded to the optimal dose of $18 \mathrm{mg}$ to 20 $\mathrm{mg}$ twice a day. It has been demonstrated that increasing the duration of rinsing with $0.12 \%$ or $0.2 \%$ chlorhexidine mouthrinse from 30 to 60 seconds results in greater substantivity of the rinse (Tomás 2010). The longer duration of rinsing employed by most of the studies in this review that evaluated $0.2 \%$ chlorhexidine mouthrinse could be expected to translate into greater retention of chlorhexidine in these studies and hence a greater effect on gingivitis and plaque. However, $75 \%$ of the chlorhexidine that is retained after rinsing for 60 seconds with $0.2 \%$ chlorhexidine rinse is retained after the first 30 seconds of rinsing (Bonesvoll 1974) and a rinse duration of 30 seconds for $0.12 \%$ chlorhexidine mouthrinse has been shown to be both effective and acceptable (Keijser 2003). Therefore, most of the studies included in this review used the optimal dose of chlorhexidine for at least 30 seconds which may partly explain why we did not find any difference in effect between the different concentration rinses. The two included studies that provided data for a direct comparison between $0.2 \%$ and $0.1 \% / 0.12 \%$ chlorhexidine rinse found no difference in effect between the different concentration rinses used with the same volume and duration of rinsing (Axelsson 1987; Segreto 1986).

Studies that evaluated the effectiveness of chlorhexidine mouthrinse on gingivitis using a cross-over study design were excluded from this review. This was due to concerns that the duration of the washout phase between the trial periods might not be sufficient to rule out a carry-over effect due to chlorhexidine mouthrinse. Carry-over can threaten the internal validity of a cross-over study and is of particular concern in studies involving chlorhexidine mouthrinse due to the established substantivity or prolonged effect of chlorhexidine mouthrinse. Evidence-based guidance on the appropriate duration of washout phase is absent from the literature and the washout periods commonly used appear to range from 2 days to 3 weeks (Berchier 2010; Newcombe 1995). The very limited data we have in this review regarding gingivitis and plaque outcomes recorded after cessation 
of chlorhexidine mouthrinsing suggest a residual effect on these outcomes weeks or months after rinsing with chlorhexidine has ceased. Although no firm conclusions can be drawn regarding the appropriateness of using a cross-over study design when the study includes a chlorhexidine mouthrinse arm, the uncertainty surrounding carry-over in relation to chlorhexidine mouthrinse warrants further investigation.

Low concentration $(0.05 \% / 0.06 \%)$ chlorhexidine mouthrinses are currently marketed for long-term daily use. Side-effects associated with chlorhexidine mouthrinse use are dose-dependant and tend to decrease when the concentration is lowered (Cumming and Löe 1973; Flotra 1971). Therefore in lowering the concentration of chlorhexidine, it is hoped that side effects will be reduced while maintaining the effect on gingivitis and plaque (Keijser 2003). Only five studies that evaluated chlorhexidine concentrations less than $0.1 \%$ met the inclusion criteria for this review. Two studies evaluated 0.05\% (Jayaprakash 2007; Joyston-Bechal 1993) and three studies evaluated $0.06 \%$ chlorhexidine mouthrinse (Hoffmann 2001; Zimmer 2006; Zimmer 2015). Although all of these studies demonstrated a reduction in plaque, the effect on gingivitis was not clear. This might be explained by the lower concentration rinses having a mainly bacteriostatic action that may produce an effect below the threshold required to produce a corresponding reduction in gingivitis. Despite the reduced concentration of chlorhexidine, extrinsic tooth staining occurred in all four studies of low concentration chlorhexidine mouthrinse that reported a staining outcome (Hoffmann 2001; Joyston-Bechal 1993; Zimmer 2006; Zimmer 2015). There has been some debate about the mechanism by which chlorhexidine mouthrinse produces extrinsic tooth staining. However, it is currently understood to be a result of precipitation of anionic dietary chromogens onto adsorbed chlorhexidine cations (Watts 2001) and thus the mechanism by which chlorhexidine mouthrinse produces extrinsic tooth staining appears to be closely linked to its mechanism of action. Therefore lack of staining is likely to indicate lack of effectiveness (Addy 2005; Eley 1999). Some degree of tooth staining can therefore be expected to feature even with low chlorhexidine concentration rinses. However, with the exception of Zimmer 2006, there were no reports of other adverse effects relating to the low concentration chlorhexidine mouthrinse apart from staining of the teeth and oral tissues.

Use of chlorhexidine mouthrinse is associated with a number of adverse effects, the most common being extrinsic staining of the teeth and oral soft tissues (Addy 1986). In many of the studies included in this review, adverse effects were inadequately reported or not reported at all. It is important that adverse effects due to chlorhexidine mouthrinse use are reported alongside evidence for effectiveness with enough detail to allow health professionals and the public to make an informed decision about recommending, prescribing or using the mouthrinse.

From our included studies it was clear that rinsing with any concentration of chlorhexidine mouthrinse for 4 weeks or longer was associated with an increase in extrinsic tooth staining. A number of the included studies reported higher levels of calculus in the chlorhexidine rinse arms but the meta-analyses were inconclusive. Other adverse effects such as taste disturbance/ alteration, burning sensation and pigmentation of the oral soft tissues were also reported in a number of included studies. Effects on the oral mucosa such as mucosal ulceration, lesions or erosions and a transient anaesthetic sensation, hypoaesthesia and paraesthesia were also reported in a number of included studies and can be considered more severe but the proportion of participants experiencing these adverse effects in any study tended to be low. While extrinsic tooth staining and calculus build-up due to chlorhexidine mouthrinse use must be removed by professional tooth cleaning incurring the costs associated with a visit to a dental professional, the other associated adverse effects are known to be transient in nature and to resolve once mouthrinsing ceases (Flotra 1971; Greenstein 1986). However, these adverse effects, although transient, can be severe and development of extrinsic tooth staining with short-term use of the mouthrinse is an obvious deterrent to using chlorhexidine mouthrinse.

\section{Quality of the evidence}

Although all the included studies were randomised controlled trials (RCTs), 50 of the 51 included studies were assessed as at high risk of bias overall, as they had at least one domain rated at high risk of bias. The reason many of the studies were assessed as at high risk of bias was due to problems with the blinding of participants, personnel, and outcome assessors. The majority of studies contained a placebo arm with the intention of blinding participants and personnel. However, we considered blinding unlikely to be successful due to the propensity for chlorhexidine to stain the teeth and oral tissues and the association of its use with clinically visible adverse effects such as mucosal desquamation and mucosal lesions. This also applied to the blinding of outcome assessors, in trials with either a placebo, control, or mechanical oral hygiene alone. This was not the fault of the investigators who in many studies went to great lengths to try to ensure blinding. Consequently we assessed 42 (82\%) studies as at high risk of bias for either sort of blinding bias (performance bias or detection bias).

Thirty-seven studies were rated at high risk of selective reporting bias, mainly due to lack of reporting of measures of variance along with the means for each group, and no reporting of tooth staining and other adverse effects.

It is worth mentioning that 22 of the included studies were published prior to the first publication of the first iteration of the CONSORT Statement in 1996 (Begg 1996) and their quality was therefore partly judged according to reporting standards that did not exist at the time of their publication. An additional nine studies were published prior to the publication of the revised CONSORT Statement in 2001 (Moher 2001). However, disappointingly, instances of poor reporting were not confined to the studies that predated widespread awareness of the CONSORT Statement.

Despite most studies included in the meta-analyses being assessed as at high risk of bias we did not downgrade the GRADE assessments for gingivitis and plaque for this reason. This is because we believe that further research is very unlikely to change our confidence in the estimate of effect for these outcomes. The grade assessments for the body of evidence for chlorhexidine mouthrinse compared with placebo, control or mechanical oral hygiene alone for gingivitis measured using the Löe and Silness Gingival Index and for plaque measured using various indices were therefore rated as high quality. Although there was high heterogeneity for some of these meta-analyses we did not downgrade for this due to consistency of the direction of effect. 
Over half of the included studies were either funded directly, received support for the study or were affiliated in some way with industry. Although a high level of industry funding is far from ideal, the reality is that without industry funding few trials evaluating the effectiveness of chlorhexidine mouthrinse would have been conducted. However, it is noteworthy that of the 19 included studies funded directly by industry, only one study (Zimmer 2015) stated that the conduct of the research and final decisions regarding the study report were independent of the company funding the research.

\section{Potential biases in the review process}

In order to prevent judgements about the eligibility criteria for studies being influenced by prior knowledge of the study results, the methods to be used in the review should be established and documented in advance of the review being undertaken (Higgins 2011). We made a number of changes to the protocol in relation to the types of studies and types of participants to be included in the review. We also clarified additional criteria for the inclusion of studies based on type of intervention that were implicit but not explicitly stated in the protocol. We amended the assessment of the blinding domains in the 'Risk of bias' tool to allow studies to obtain a judgement of 'low' or 'unclear' risk of bias in certain circumstances compared to the blanket judgement of high risk of bias that was stated in the published protocol.

A hierarchy to guide data extraction for gingivitis and plaque data was developed to facilitate data extraction and analysis. The main analysis however, was based on the main prespecified gingivitis index: the Gingival Index of Löe and Silness (Löe 1967; Löe and Silness 1963). Full details of the changes to the protocol are described in the Differences between protocol and review section. It is acknowledged that it may not always be appropriate to adhere to the published protocol (Higgins 2011). Therefore, while these changes to the protocol could introduce a risk of bias, the changes were made in the early stages of the review before any analyses of the results were undertaken and are therefore considered unlikely to have been influenced by knowledge of the study results or the results of the review. Furthermore, each decision was appropriately justified and was made to improve the scientific quality and clinical applicably of the review.

We estimated the standard deviations for four studies that measured the Gingival Index at 6 months (Banting 1989; Jayaprakash 2007; Lucas 1999; Sanz 1994) and one study that measured the Gingival Index at 4 to 6 weeks (Sanz 1989) using data from the same outcomes measured at the same time point in other similar studies so that we could include these data in meta-analyses. Where mean scores or measures of variance of the mean scores or both were not reported in the text or in tabular form, and could not be obtained from the authors of the studies, data were estimated by reading them off the graphs in the published report for inclusion in meta-analyses for six studies (Brecx 1993; Brightman 1991; Chaves 1994; Ferretti 1987; Jenkins 1993; Joyston-Bechal 1993). The graphs were enlarged and the data were estimated independently and in duplicate. A sensitivity analysis for the Gingival Index at both 4 to 6 weeks and 6 months, excluding trials where we had to estimate the standard deviation from similar studies, or had to read the data from graphs, showed that the effect estimates were similar to those for the overall pooling of the studies.

\section{Agreements and disagreements with other studies or reviews}

Van Strydonck 2012 conducted a comprehensive review of the effect of chlorhexidine mouthrinse on plaque, gingival inflammation and tooth staining in studies of 4 weeks or longer. This review was very similar to our review but slight differences in the inclusion criteria and the dates of the literature searches meant that a slightly different group of studies were reviewed by Van Strydonck et al. The review focused on healthy adults only, considered chlorhexidine mouthrinse used as a monotherapy or as an adjunct, and excluded patients with periodontitis or dentures as well as orthodontic and implant patients. In addition, apart from tooth staining, adverse effects although reported in the review, were not prespecified outcomes. Of the 30 studies included in this review, 27 are common to our review. The results of the metaanalysis of studies reporting the $\mathrm{Gl}$ at all time points included data from seven studies, six of which were included in our metaanalyses at either 4 to 6 weeks, 7 to 12 weeks or 6 months. The weighted mean difference for the $\mathrm{Gl}$ in this review was -0.32 which is similar to the mean difference for the GI reported in our review. Extrinsic tooth staining, increased calculus formation and change of taste sensation were also noted to have frequently occurred in the included studies. Considerable heterogeneity in the design, study duration and rinse regimen were also noted to be present.

Boyle 2014 systematically reviewed the evidence relating to the efficacy of the most frequently used mouthrinses for the control of supragingival plaque and gingivitis. Both the scope of the literature search and the inclusion criteria were narrower than in our review. Of the 17 trials involving chlorhexidine mouthrinse included in this review 16 are common to our review. The reported 'summary relative differences' facilitated comparison between the different mouthrinse preparations being reviewed. Results for the chlorhexidine mouthrinse studies are supportive of the effectiveness of chlorhexidine mouthrinse primarily for the control of both gingivitis and plaque relative to control but also relative to other active mouthrinse preparations.

A systematic review by Serrano 2015 evaluated the effectiveness of adjunctive antiplaque chemical agents including chlorhexidine mouthrinse in RCTs of at least 6 months duration. The review included 14 studies evaluating chlorhexidine mouthrinse. Due to differences in inclusion criteria, 12 of these studies are common to our review. The authors reported a significant benefit for rinsing with $0.1 \%$ to $0.2 \%$ chlorhexidine mouthrinse based on a metaanalysis of four studies (weighted mean difference on the GI -0.185 ) but a non-significant effect for chlorhexidine mouthrinse at concentrations of $\leq 0.06 \%$. In common with our review was the presence of high heterogeneity which made it difficult to pool the data and was an acknowledged limitation of the review.

Gunsolley 2006 conducted a meta-analysis of 6-month mouthrinse studies for the control of gingivitis and plaque. Six of the included studies evaluated the effect of $0.12 \%$ chlorhexidine mouthrinse using the $\mathrm{Gl}$ and all six were common to our review. The analysis indicated a moderate effect for gingivitis and a large effect for plaque. A subsequent metareview also conducted by Gunsolley drew on the data from the earlier meta-analysis and found a weighted mean percentage reduction of $28.7 \%$ for the $\mathrm{Gl}$ and $40.4 \%$ for the Plaque Index (Gunsolley 2010) based on the same six studies. 
The efficacy of $0.12 \%$ chlorhexidine mouthrinse compared to $0.2 \%$ on plaque and periodontal parameters was systematically reviewed by Berchier 2010. The review included eight studies with rinse duration from 3 days to 3 months that directly compared $0.12 \%$ with $0.2 \%$ chlorhexidine mouthrinse. The review found no difference in the effect on gingivitis between the two concentrations of chlorhexidine. The small significant difference in plaque inhibition in favour of $0.2 \%$ chlorhexidine was thought to be of negligible clinical relevance. Our review included two studies that provided data for a direct comparison between $0.2 \%$ and $0.1 \% / 0.12 \%$ chlorhexidine rinse (Axelsson 1987; Segreto 1986), one of which was also included in the Berchier review (Segreto 1986). There was no difference in effect between the different concentration rinses used with the same volume and duration of rinsing in these two studies. Similarly our subgroup analysis found no evidence that one concentration of chlorhexidine rinse was more effective than another in reducing gingivitis and plaque.

van der Weijden 2015 conducted a metareview of the evidence regarding the efficacy and safety of chemical agents in mouthrinses to manage gingivitis and plaque. The evidence for chlorhexidine mouthrinse was drawn from the systematic reviews (Serrano 2015; Van Strydonck 2012) and meta-analyses (Gunsolley 2006; Gunsolley 2010) described above. Berchier 2010 contributed data for the comparison of active ingredients. This review reaffirmed the effectiveness of chlorhexidine mouthrinse for reducing plaque and gingivitis. The body of evidence resulting from these three reviews was rated as 'strong'. Due to the heterogeneity in the meta-analyses, the authors advised caution in interpreting the difference of means as it "may not provide an exact measure of the results" (van der Weijden 2015). Although the authors concluded that chlorhexidine is the "first choice", they also acknowledged that the development of taste disturbance, tooth staining and calculus formation prohibit long-term adjunctive use of the mouthrinse.

\section{AUTHORS' CONCLUSIONS}

\section{Implications for practice}

This review found high-quality evidence of a large reduction in dental plaque with chlorhexidine mouthrinse used as an adjunct to mechanical oral hygiene procedures for 4 to 6 weeks and 6 months. We also found high-quality evidence from studies that reported the Löe and Silness Gingival Index of a reduction in gingivitis in individuals with mild gingival inflammation on average (mean score of 1 on the 0 to 3 Gingival Index (GI) scale), that was not considered to be clinically relevant. There was no evidence that one concentration of chlorhexidine mouthrinse was more effective than another. There was insufficient evidence to determine the reduction in gingivitis associated with chlorhexidine mouthrinse use in individuals with mean Gl scores of 1.1 to 3 indicating moderate or severe levels of gingival inflammation. Rinsing with chlorhexidine mouthrinse for 4 weeks or longer causes extrinsic tooth staining. In addition, other adverse effects such as calculus build-up, transient taste disturbance and effects on the oral mucosa were reported in the included studies.Chlorhexidine mouthrinse is indicated in particular clinical situations for short periods of time. Using chlorhexidine mouthrinse for longer periods of time in individuals with special care needs who cannot maintain an adequate level of plaque control using mechanical cleaning methods alone must be carefully weighed against the adverse effects associated with its use.

\section{Implications for research}

The majority of the studies included in the main analysis of the Gingival Index at 4 to 6 weeks and 6 months involved healthy participants with low levels of gingivitis.There was insufficient evidence to determine the reduction in gingivitis associated with chlorhexidine mouthrinse use in individuals with mean $\mathrm{Gl}$ scores of 1.1 to 3 indicating moderate or severe levels of gingival inflammation. Some of the studies included in the metaanalyses of gingival bleeding at 4 to 6 weeks and 6 months included participants with a high proportion of sites with bleeding on probing. However, due to the variety of different gingival bleeding indices employed, we were unable to determine the mean reduction in gingivitis associated with chlorhexidine mouthrinse use in individuals with moderate or severe levels of gingival inflammation. Further well-conducted randomised controlled trials are needed to investigate the effect size for adjunctive chlorhexidine mouthrinse use in individuals with moderate or severe levels of gingival inflammation. Agreement among the experts about which indices are most appropriate for measuring gingivitis in studies evaluating antimicrobial mouthrinses would facilitate future evidence syntheses. Evidence-based guidance regarding patient selection for chlorhexidine mouthrinse use would help to provide clarity regarding the 'particular clinical situations' in which short-term use of chlorhexidine mouthrinse is recommended.

Only five studies evaluating $0.05 \%$ or $0.06 \%$ chlorhexidine mouthrinse met the inclusion criteria for this review. These studies demonstrated a reduction in plaque but the effect of these low-concentration mouthrinses on gingivitis was not clear or consistent. As low-concentration chlorhexidine mouthrinse is currently marketed for long-term daily use, well-conducted randomised controlled trials are needed to investigate the effect of these mouthrinses on gingivitis and to record any adverse effects associated with their use.

Due to the unavoidable influence of extrinsic tooth staining associated with use of chlorhexidine mouthrinse on blinding of participants, personnel and outcome assessors, particular attention must be given to robust study design and rigorous reporting of the other domains in the Cochrane 'Risk of bias' tool. In particular, estimates of effect should be fully reported with a measure of variance so that data can be included in meta-analyses. Evidence of adverse effects should also be fully reported for each study arm. The CONSORT Statement (Schulz 2010) should be used to optimise reporting in future studies. The appropriateness of using studies with cross-over design that include chlorhexidine mouthrinse warrants further investigation.

\section{ACKNOWLEDGEMENTS}

We wish to thank Ms Anne Littlewood, Information Specialist, Cochrane Oral Health, for developing the search strategy, conducting the database searches and writing up the 'Electronic searches' section of the review; and Luisa Fernandez Mauleffinch, Managing Editor and Copy Editor, Cochrane Oral Health, for her assistance with the editorial process. We thank Anne-Marie Glenny and Jacopo Buti for their comments on the draft. We would like to thank Leyes Borrajo, Michel Brecx, Jack Caton, Eros Chaves, Dan Ericson, Thomas Flemmig, Peter Fine, Stuart Fischman, Per Gjermo, Michael Newman, Mariano Sanz, and Francesco Spadari for responding to our queries regarding their studies; and Magda 
Feres, Marcelo de Faveri, Jan Hase and Torgny Sjödin for providing additional outcome data from their studies. We would also like to thank Irene Halpin, Cork University Hospital Medical Library and the staff at the Interlibrary Loans Department in the Boole Library, University College Cork for their assistance in obtaining many of the studies for this review through interlibrary loans and stores requests. We would like to thank Makiko Nishi (Oral Health Services
Research Centre) for her assistance with obtaining unpublished data and assistance with assessment of eligibility of Japanese and Chinese papers and the staff of the Oral Health Services Research Centre, Cork for their support and encouragement. Patrice James would like to thank Mark, Oisin, Aoife and Rian for their love and unfailing support. 


\section{REFERE N CES}

\section{References to studies included in this review}

Anauate-Netto 2014 \{published data only\}

Anauate-Netto C, Anido-Anido A, Leegoy HR, Matsumoto R, Alonso RC, Marcucci MC, et al. Randomized, double-blind, placebo-controlled clinical trial on the effects of propolis and chlorhexidine mouthrinses on gingivitis. Brazilian Dental Science 2014;17(1):11-5.

\section{Anderson 1997 \{published data only\}}

Anderson GB, Bowden J, Morrison EC, Caffesse RG. Clinical effects of chlorhexidine mouthwashes on patients undergoing orthodontic treatment. American Journal of Orthodontics and Dentofacial Orthopedics 1997;111(6):606-12.

\section{Axelsson 1987 \{published data only\}}

Axelsson P, Lindhe J. Efficacy of mouthrinses in inhibiting dental plaque and gingivitis in man. Journal of Clinical Periodontology 1987;14(4):205-12.

\section{Bajaj 2011 \{published data only\}}

Bajaj N, Tandon S. The effect of Triphala and Chlorhexidine mouthwash on dental plaque, gingival inflammation, and microbial growth. International Journal of Ayurveda Research 2011;2(1):29-36

\section{Banting 1989 \{published data only\}}

Banting D, Bosma M, Bollmer B. Clinical effectiveness of a $0.12 \%$ chlorhexidine mouthrinse over two years. Journal of Dental Research 1989;68(Spec Iss):1716-8.

\section{Bhat 2014 \{published data only\}}

Bhat N, Mitra R, Oza S, Mantu Vinayak K, Bishnoi S, Gohil M, et al. The antiplaque effect of herbal mouthwash in comparison to chlorhexidine in human gingival disease: a randomized placebo controlled clinical trial. Journal of Complementary and Integrative Medicine 2014;11(2):129-37.

\section{Brecx 1993 \{published data only\}}

Brecx M, Macdonald LL, Legary K, Cheang M, Forgay MG. Longterm effects of Meridol and chlorhexidine mouthrinses on plaque, gingivitis, staining, and bacterial vitality. Journal of Dental Research 1993;72(8):1194-7.

\section{Brightman 1991 \{published data only\}}

Brightman LJ, Terezhalmy GT, Greenwell H, Jacobs M, Enlow DH. The effects of a $0.12 \%$ chlorhexidine gluconate mouthrinse on orthodontic patients aged 11 through 17 with established gingivitis. American Journal of Orthodontics and Dentofacial Orthopedics 1991;100(4):324-9.

\section{Charles 2004 \{published data only\}}

Charles CH, Mostler KM, Bartels LL, Mankodi SM. Comparative antiplaque and antigingivitis effectiveness of a chlorhexidine and an essential oil mouthrinse: 6-month clinical trial. Journal of Clinical Periodontology 2004;31(10):878-84.
Chaves 1994 \{published data only\}

* Chaves ES, Kornman KS, Manwell MA, Jones AA, Newbold DA, Wood RC. Mechanism of irrigation effects on gingivitis. Journal of Periodontology 1994;65(11):1016-21.

Chaves ES, Wood RC, Jones AA, Newbold DA, Manwell MA, Kornman KS. Relationship of "bleeding on probing" and "gingival index bleeding" as clinical parameters of gingival inflammation. Journal of Clinical Periodontology 1993;20(2):139-43.

\section{Corbet 1997 \{published data only\}}

Corbet EF, Tam JO, Zee KY, Wong MC, Lo EC, Mombelli AW, et al. Therapeutic effects of supervised chlorhexidine mouthrinses on untreated gingivitis. Oral Diseases 1997;3(1):9-18.

\section{de la Rosa 1988 \{published data only\}}

de la Rosa M, Sturzenberger OP, Moore DJ. The use of chlorhexidine in the management of gingivitis in children. Journal of Periodontology 1988;59(6):387-9.

de la Rosa 1988b \{published data only\} de la Rosa M, Sturzenberger OP, Moore DJ. The use of chlorhexidine in the management of gingivitis in children. Journal of Periodontology 1988;59(6):387-9.

\section{Eaton 1997 \{published data only\}}

Eaton KA, Rimini FM, Zak E, Brookman DJ, Hopkins LM, Cannell PJ, et al. The effects of a $0.12 \%$ chlorhexidinedigluconate-containing mouthrinse versus a placebo on plaque and gingival inflammation over a 3-month period. A multicentre study carried out in general dental practices. Journal of Clinical Periodontology 1997;24(3):189-97.

\section{Emling 1992 \{published data only\}}

Emling RC, Levin S, Shi X, Weinberg S, Yankell SL. Rembrandt toothpaste stain prevention with and without the use of Peridex. Journal of Clinical Dentistry 1992;3(2):59-65.

\section{Ernst 2005 \{published data only\}}

Ernst CP, Canbek K, Dillenburger A, Willershausen B. Clinical study on the effectiveness and side effects of hexetidine and chlorhexidine mouthrinses versus a negative control. Quintessence International 2005;36(8):641-52.

\section{Faveri 2006 \{published and unpublished data\}}

Faveri M, Gursky LC, Feres M, Shibli JA, Salvador SL, de Figueiredo LC. Scaling and root planing and chlorhexidine mouthrinses in the treatment of chronic periodontitis: a randomized, placebo-controlled clinical trial. Journal of Clinical Periodontology 2006;33(11):819-28.

\section{Feres 2009 \{published and unpublished data\}}

Feres M, Gursky LC, Faveri M, Tsuzuki CO, Figueiredo LC. Clinical and microbiological benefits of strict supragingival plaque control as part of the active phase of periodontal therapy. Journal of Clinical Periodontology 2009;36(10):857-67. 


\section{Feres 2012 \{published and unpublished data\}}

Feres M, Soares GM, Mendes JA, Silva MP, Faveri M, Teles R, et al. Metronidazole alone or with amoxicillin as adjuncts to nonsurgical treatment of chronic periodontitis: a 1-year doubleblinded, placebo-controlled, randomized clinical trial. Journal of Clinical Periodontology 2012;39(12):1149-58.

\section{Ferretti 1987 \{published data only\}}

Ferretti GA, Ash RC, Brown AT, Largent BM, Kaplan A, Lillich TT. Chlorhexidine for prophylaxis against oral infections and associated complications in patients receiving bone marrow transplants. Journal of the American Dental Association 1987;114(4):461-7.

\section{Fine 1985 \{published and unpublished data\}}

Fine PD. A clinical trial to compare the effect of two antiseptic mouthwashes on gingival inflammation. Journal of Hospital Infection 1985;6(Suppl A):189-93.

\section{Flemmig 1990 \{published and unpublished data\}}

Flemmig TF, Newman MG, Doherty FM, Grossman E, Meckel AH, Bakdash MB. Supragingival irrigation with $0.06 \%$ chlorhexidine in naturally occurring gingivitis. I. 6 month clinical observations. Journal of Periodontology 1990;61(2):112-7.

\section{Flotra 1972 \{published and unpublished data\}}

* Flotra L, Gjermo P, Rolla G, Waerhaug J. A 4-month study on the effect of chlorhexidine mouth washes on 50 soldiers. Scandinavian Journal of Dental Research 1972;80(1):10-7.

Flotra L, Gjermo P, Rolla G, Waerhaug J. Side effects of chlorhexidine mouth washes. Scandinavian Journal of Dental Research 1971;79(2):119-25.

\section{Graziani 2015 \{published data only\}}

Graziani F, Gabriele M, D'Aiuto F, Suvan J, Tonelli M, Cei S. Dental plaque, gingival inflammation and tooth -discolouration with different commercial -formulations of $0.2 \%$ chlorhexidine rinse: a double-blind randomised controlled clinical trial. Oral Health and Preventive Dentistry 2015;13(2):101-11.

\section{Grossman 1986 \{published data only\}}

Grossman E, Reiter G, Sturzenberger OP. Six-month study of the effects of a chlorhexidine mouthrinse on gingivitis in adults. Journal of Periodontal Research 1986;21(Suppl 16):33-43.

\section{Grossman 1989 \{published data only\}}

Grossman E, Meckel AH, Isaacs RL, Ferretti GA, Sturzenberger OP, Bollmer BW, et al. A clinical comparison of antibacterial mouthrinses: effects of chlorhexidine, phenolics, and sanguinarine on dental plaque and gingivitis. Journal of Periodontology 1989;60(8):435-40.

\section{Hase 1995 \{published and unpublished data\}}

Hase JC, Ainamo J, Etemadzadeh H, Astrom M. Plaque formation and gingivitis after mouthrinsing with $0.2 \%$ delmopinol hydrochloride, $0.2 \%$ chlorhexidine digluconate and placebo for 4 weeks, following an initial professional tooth cleaning. Journal of Clinical Periodontology 1995;22(7):533-9.

\section{Hase 1998 \{published and unpublished data\}}

Hase JC, Attstrom R, Edwardsson S, Kelty E, Kisch J. 6-month use of $0.2 \%$ delmopinol hydrochloride in comparison with $0.2 \%$ chlorhexidine digluconate and placebo. (I). Effect on plaque formation and gingivitis. Journal of Clinical Periodontology 1998;25(9):746-53.

\section{Hoffmann 2001 \{published data only\}}

Hoffmann T, Bruhn G, Richter S, Netuschil L, Brecx M. Clinical controlled study on plaque and gingivitis reduction under longterm use of low-dose chlorhexidine solutions in a population exhibiting good oral hygiene. Clinical Oral Investigations 2001;5(2):89-95.

\section{Jayaprakash 2007 \{published data only\}}

Jayaprakash K, Veeresha KL, Hiremath SS. A comparative study of two mouthrinses on plaque and gingivitis in school children in the age group of 13-16 years in Bangalore city. Journal of the Indian Society of Pedodontics and Preventive Dentistry 2007;25(3):126-9.

\section{Jenkins 1993 \{published data only\}}

Jenkins S, Addy M, Newcombe R. Evaluation of a mouthrinse containing chlorhexidine and fluoride as an adjunct to oral hygiene. Journal of Clinical Periodontology 1993;20(1):20-5.

\section{Jose 2015 \{published data only\}}

Jose A, Butler A, Payne D, Maclure R, Rimmer P, Bosma ML. A randomised clinical study to evaluate the efficacy of alcoholfree or alcohol-containing mouthrinses with chlorhexidine on gingival bleeding. British Dental Journal 2015;219(3):125-30, 6.

\section{Joyston-Bechal 1993 \{published data only\}}

Joyston-Bechal S, Hernaman N. The effect of a mouthrinse containing chlorhexidine and fluoride on plaque and gingival bleeding. Journal of Clinical Periodontology 1993;20(1):49-53.

\section{Lang 1998 \{published and unpublished data\}}

Lang NP, Hase JC, Grassi M, Hammerle CH, Weigel C, Kelty E, et al. Plaque formation and gingivitis after supervised mouthrinsing with $0.2 \%$ delmopinol hydrochloride, $0.2 \%$ chlorhexidine digluconate and placebo for 6 months. Oral Diseases 1998;4(2):105-13.

\section{Lopez-Jornet 2012 \{published data only\}}

Lopez-Jornet P, Plana-Ramon E, Leston JS, Pons-Fuster A. Short-term side effects of $0.2 \%$ alcohol-free chlorhexidine mouthrinse in geriatric patients: a randomized, double-blind, placebo-controlled study. Gerodontology 2012;29(4):292-8.

\section{Lucas 1999 \{published data only\}}

Lucas GQ, Lucas ON. Preventive action of short-term and longterm chlorhexidine rinses. Acta Odontologica Latinoamericana 1999;12(1):45-58

\section{Navarro 1998 \{published data only\}}

Navarro D, Santos EAT, Rocha JCF, Bremm LL, Jukoski M, Ribeiro PG, et al. Effect of chlorhexidine digluconate, Plantago major and placebo mouth rinse on dental plaque and gingivitis [Efeitos do digluconato de clorexidina, Plantago major e placebo sobre placa dental e gengivite: uma comparacao 
clinica da eficacia de colutorios]. Revista Brasileira de Plantas Medicinais 1998;1(1):28.

\section{Overholser 1990 \{published data only\}}

Overholser CD, Meiller TF, DePaola LG, Minah GE, Niehaus C. Comparative effects of 2 chemotherapeutic mouthrinses on the development of supragingival dental plaque and gingivitis. Journal of Clinical Periodontology 1990;17(8):575-9.

\section{Pereira 2011 \{published data only\}}

Pereira SL, de Oliveira JW, Angelo KK, da Costa AM, Costa F. Clinical effect of a mouth rinse containing Ocimum gratissimum on plaque and gingivitis control. Journal of Contemporary Dental Practice 2011;12:350-5.

\section{Rahmani 2006 \{published data only\}}

Rahmani ME, Radvar M. Effects of chlorhexidine (0.2\%) as irrigant during ultrasonic debridement: A clinical study. International Journal of Pharmacology 2006;2(1):89-92.

\section{Sanz 1989 \{published data only\}}

Sanz M, Newman MG, Anderson L, Matoska W, Otomo-Corgel J, Saltini C. Clinical enhancement of post-periodontal surgical therapy by a $0.12 \%$ chlorhexidine gluconate mouthrinse. Journal of Periodontology 1989;60(10):570-6.

\section{Sanz 1994 \{published data only\}}

Sanz M, Vallcorba N, Fabregues S, Muller I, Herkstroter F. The effect of a dentifrice containing chlorhexidine and zinc on plaque, gingivitis, calculus and tooth staining. Journal of Clinical Periodontology 1994;21(6):431-7.

\section{Segreto 1986 \{published data only\}}

Segreto VA, Collins EM, Beiswanger BB. A comparison of mouthrinses containing two concentrations of chlorhexidine. Journal of Periodontal Research 1986;21(Suppl 16):23-32.

\section{Southern 2006 \{published data only\}}

Southern EN, McCombs GB, Tolle SL, Marinak K. The comparative effects of $0.12 \%$ chlorhexidine and herbal oral rinse on dental plaque-induced gingivitis. Journal of Dental Hygiene 2006;80(1):12.

\section{Stookey 2005 \{published data only\}}

Stookey GK, Beiswanger B, Mau M, Isaacs RL, Witt JJ, Gibb R. A 6-month clinical study assessing the safety and efficacy of two cetylpyridinium chloride mouthrinses. American Journal of Dentistry 2005;18(Spec No):24A-8A.

Taller 1993 \{published data only\}

Taller SH. The effect of baking soda/hydrogen peroxide dentifrice (Mentadent) and a 0.12 percent chlorhexidine gluconate mouthrinse (Peridex) in reducing gingival bleeding. Journal of the New Jersey Dental Association 1993;64(4):23-5.

Turkoglu 2009 \{published data only\}

Turkoglu O, Becerik S, Emingil G, Kutukculer N, Baylas H, Atilla G. The effect of adjunctive chlorhexidine mouthrinse on clinical parameters and gingival crevicular fluid cytokine levels in untreated plaque-associated gingivitis. Inflammation Research 2009;58(5):277-83.

\section{Van Strydonck 2008 \{published data only\}}

Van Strydonck DA, Timmerman MF, Van der Velden U, Van der Weijden F. Clinical efficacy of a chlorhexidinedelivering toothbrush. Journal of Clinical Periodontology 2008;35(7):584-90.

\section{Weitz 1992 \{published data only\}}

Weitz M, Brownstein C, Deasy M. Effect of a twice daily $0.12 \%$ chlorhexidine rinse on the oral health of a geriatric population. Clinical Preventive Dentistry 1992;14(3):9-13.

\section{Zimmer 2006 \{published data only\}}

Zimmer S, Kolbe C, Kaiser G, Krage T, Ommerborn M, Barthel C. Clinical efficacy of flossing versus use of antimicrobial rinses. Journal of Periodontology 2006;77(8):1380-5.

Zimmer 2015 \{published data only\}

Zimmer S, Korte P, Verde P, Ohmann C, Naumova E, Jordan RA. Randomized controlled trial on the efficacy of new alcohol-free chlorhexidine mouthrinses after 8 weeks. International Journal of Dental Hygiene 2015;13(2):110-6.

\section{References to studies excluded from this review}

Bay 1975 \{published data only\}

Bay LM, Russell BG. Effect of chlorhexidine on dental plaque and gingivitis in mentally retarded children. Community Dentistry and Oral Epidemiology 1975;3(6):267-70.

\section{Biswas 2014 \{published data only\}}

Biswas G, Anup N, Acharya S, Kumawat H, Vishnani P, Tambi S. Evaluation of the efficacy of $0.2 \%$ chlorhexidine versus herbal oral rinse on plaque induced gingivitis- A randomised clinical trial. Journal of Nursing and Health Science 2014;3(2):58-63.

\section{Bouwsma 1992 \{published data only\}}

Bouwsma OJ, Yost KG, Baron HJ. Comparison of a chlorhexidine rinse and a wooden interdental cleaner in reducing interdental gingivitis. American Journal of Dentistry 1992;5(3):143-6.

\section{Brown 2002 \{published data only\}}

Brown JB, Rosenstein D, Mullooly J, O'Keeffe Rosetti M, Robinson S, Chiodo G. Impact of intensified dental care on outcomes in human immunodeficiency virus infection. AIDS Patient Care and STDs 2002;16(10):479-86.

Caton 1993 \{published data only (unpublished sought but not used)\}

Caton JG, Blieden TM, Lowenguth RA, Frantz BJ, Wagener CJ, Doblin JM, et al. Comparison between mechanical cleaning and an antimicrobial rinse for the treatment and prevention of interdental gingivitis. Journal of Clinical Periodontology 1993;20(3):172-8

\section{Cortelli 2015 \{published data only\}}

Cortelli Sheila C, Costa Fernando O, Rodrigues E, Cota Luis OM, Cortelli Jose R. Periodontal therapy effects on nitrite related to oral bacteria: a 6-month randomized clinical trial. Journal of Periodontology 2015;86(8):984-994 11. 
CTRI 2014 \{published data only\}

CTRI/2014/10/005145. Comparison of effect of green tea mouthwash and commonly prescribed mouthwash(chlorhexidine) in reducing gum inflammation. www.ctri.nic.in/Clinicaltrials/ (first received 27 October 2014).

\section{Ernst 1998 \{published data only\}}

Ernst CP, Prockl K, Willershausen B. The effectiveness and side effects of $0.1 \%$ and $0.2 \%$ chlorhexidine mouthrinses: a clinical study. Quintessence International 1998;29(7):443-8.

\section{Eshwar 2016 \{published data only\}}

Eshwar S, K R, Jain V, Manvi S, Kohli S, Bhatia S. Comparison of dill seed oil mouth rinse and chlorhexidine mouth rinse on plaque levels and gingivitis - A double blind randomized clinical trial. Open Dentistry Journal 2016;10:207-13.

Fischman 1975 \{published data only (unpublished sought but not used)\}

Fischman S, Picozzi A, Cancro L, Pader M. Influence of a chlorhexidine and a zinc mouthrinse on gingivitis. Journal of Periodontology 1975;46(12):710-4.

\section{Goutham 2013 \{published data only\}}

Goutham BS, Manchanda K, De Sarkar A, Prakash R, Jha K, Mohammed S. Efficacy of two commercially available oral rinses - Chlorhexidine and Listerine on plaque and gingivitis. Journal of International Oral Health 2013;5(4):56-61.

\section{Gupta 2014 \{published data only\}}

Gupta D, Bhaskar DJ, Gupta RK, Karim B, Jain A, Singh R, et al. A randomized controlled clinical trial of Ocimum sanctum and chlorhexidine mouthwash on dental plaque and gingival inflammation. Journal of Ayurveda and Integrative Medicine 2014;5(2):109-16.

\section{Gupta 2015 \{published data only\}}

Gupta D, Gupta RK, Bhaskar DJ, Gupta V. Comparative evaluation of terminalia chebula extract mouthwash and chlorhexidine mouthwash on plaque and gingival inflammation - 4-week randomised control trial. Oral Health and Preventive Dentistry 2015;13(1):5-12.

\section{Gupta 2015a \{published data only\}}

Gupta D, Jain A. Effect of cinnamon extract and chlorhexidine gluconate $(0.2 \%)$ on the clinical level of dental plaque and gingival health: a 4-week, triple-blind randomized controlled trial. Journal of the International Academy of Periodontology 2015;17(3):91-8.

\section{Haffajee 2009 \{published data only\}}

Haffajee AD, Roberts C, Murray L, Veiga N, Martin L, Teles RP, et al. Effect of herbal, essential oil, and chlorhexidine mouthrinses on the composition of the subgingival microbiota and clinical periodontal parameters. Journal of Clinical Dentistry 2009;20(7):211-7.

\section{Karim 2014 \{published data only\}}

Karim B, Bhaskar DJ, Agali C, Gupta D, Gupta RK, Jain A, et al. Effect of aloe vera mouthwash on periodontal health: triple blind randomized control trial. Oral Health and Dental Management 2014;13(1):14-9.

\section{Lang 1982 \{published data only\}}

Lang NP, Hotz P, Graf H. Effects of supervised chlorhexidine mouthrinses in children. A longitudinal clinical trial. Journal of Periodontal Research 1982;17(1):101-11.

\section{Leyes Borrajo 2002 \{published data only\}}

Leyes Borrajo JL, Garcia VL, Lopez CG, Rodriguez-Nunez I, Garcia FM, Gallas TM. Efficacy of chlorhexidine mouthrinses with and without alcohol: a clinical study [Erratum appears in Journal of Periodontology 2006;77(5):931]. Journal of Periodontology 2002;73(3):317-21.

\section{Luoma 1978 \{published data only\}}

Luoma H, Murtomaa H, Nuuja T. A simultaneous reduction of caries and gingivitis in a group of schoolchildren receiving chlorhexidine-fluoride applications. Caries Research 1978;12(5):290-8.

\section{Madden 2008 \{published data only\}} Madden TE, Herriges B, Boyd LD, Laughlin G, Chiodo G, Rosenstein D. Alterations in $\mathrm{HbAlc}$ following minimal or enhanced non-surgical, non-antibiotic treatment of gingivitis or mild periodontitis in type 2 diabetic patients: a pilot trial. Journal of Contemporary Dental Practice 2008;9(5):9-16.

\section{McKenzie 1992 \{published data only\}}

McKenzie WT, Forgas L, Vernino AR, Parker D, Limestall JD. Comparison of a $0.12 \%$ chlorhexidine mouthrinse and an essential oil mouthrinse on oral health in institutionalized, mentally handicapped adults: one-year results. Journal of Periodontology 1992;63(3):187-93.

\section{Nadkerny 2015 \{published data only\}}

Nadkerny PV, Ravishankar PL, Pramod V, Agarwal LA, Bhandari S. A comparative evaluation of the efficacy of probiotic and chlorhexidine mouthrinses on clinical inflammatory parameters of gingivitis: A randomized controlled clinical study. Journal of Indian Society of Periodontology 2015;19(6):633-9.

\section{NCT01750801 \{published data only\}}

NCT01750801. Clinical evidence efficacy of a mouthwash containing Propolis for the control of plaque and gingivitis: Phase III, randomized, double-blind comparison with mouthwash chlorhexidine base. http://clinicaltrials.gov/show/ NCT01750801 (first received 22 November 2012).

\section{NCT02546804 \{published data only\}}

\section{NCT02546804. Evaluate the efficacy of potassium} permanganate mouthwash and hot salt water mouthwash and their comparison with chlorhexidine mouthwash on plaque index, gingival index and periodontal probing depth. https:// clinicaltrials.gov/show/NCT02546804 (first received 26 August 2015).

\section{Ousehal 2011 \{published data only\}}

Ousehal L, Lazrak L, Es-said R, Hamdoune H, Elquars F, Khadija A. Evaluation of dental plaque control in patients 
wearing fixed orthodontic appliances: a clinical study. International Orthodontics 2011;9:140-55.

\section{Paknejad 2006 \{published data only\}}

Paknejad M, Jafarzadeh TS, Shamloo AM. Comparison of the efficacy of Matrica and $\% 0.2$ chlorhexidine mouthwashes on 3-6 $\mathrm{mm}$ pockets in patient with chronic periodontitis. Journal of Islamic Dental Association of Iran 2006;18(3):16.

\section{Persson 1991 \{published data only\}}

Persson RE, Truelove EL, LeResche L, Robinovitch MR. Therapeutic effects of daily or weekly chlorhexidine rinsing on oral health of a geriatric population. Oral Surgery, Oral Medicine and Oral Pathology 1991;72(2):184-91.

\section{Persson 1998 \{published data only\}}

Persson RE, Persson GR, Powell LV, Kiyak HA. Periodontal effects of a biobehavioral prevention program. Journal of Clinical Periodontology 1998;25(4):322-9.

\section{Priya 2015 \{published data only\}}

Priya BM, Galgali SR. Comparison of amine fluoride and chlorhexidine mouth rinses in the control of plaque and gingivitis - a randomized controlled clinical trial. Indian Journal of Dental Research 2015;26(1):57-62.

\section{Quirynen 2005 \{published data only\}}

Quirynen M, Soers C, Desnyder M, Dekeyser C, Pauwels M, van Steenberghe D. A 0.05\% cetyl pyridinium chloride/0.05\% chlorhexidine mouth rinse during maintenance phase after initial periodontal therapy. Journal of Clinical Periodontology 2005;32(4):390-400.

\section{Quirynen 2006 \{published data only\}}

Quirynen M, Teughels W, van Steenberghe D. Impact of antiseptics on one-stage, full-mouth disinfection. Journal of Clinical Periodontology 2006;33(1):49-52.

\section{Radafshar 2017 \{published data only\}}

Radafshar G, Ghotbizadeh M, Saadat F, Mirfarhadi N. Effects of green tea (Camellia sinensis) mouthwash containing 1\% tannin on dental plaque and chronic gingivitis: a doubleblinded, randomized, controlled trial. Journal of Investigative and Clinical Dentistry 2017;8:(1).

\section{Saltini 1988 \{published data only\}}

Saltini C, Sanz M, Newman MG, Doherty F. Clinical efficacy of a $0.12 \%$ chlorhexidine mouthrinse in periodontal maintenance patients with persistent disease. Journal of Dental Research 1988;67(Spec Iss, IADR, AADR, CADR March 9-13 1988):183 (Abs No 567).

\section{Segreto 1993 \{published data only\}}

Segreto V, Collins E. A clinical investigation to assess the effects on plaque, gingivitis, and staining potential of an experimental mouthrinse (research report on file). Cincinnati: Procter \& Gamble; 1993. Report No: 002392.

\section{Subhash 1985 \{published data only\}}

Subhash G, Das AK. The effect of chlorhexidine gluconate solution on gingivitis and its best mode of application. Journal of the Indian Dental Association 1985;57(12):501-4.

Todkar 2012 \{published data only\}

Todkar R, Sheikh S, Byakod G, Muglikar S. Efficacy of chlorhexidine mouthrinses with and without alcohol-A clinical study. Oral Health and Preventive Dentistry 2012;10:291-6.

Vechis-Bon 1989 \{published data only\}

Vechis-Bon S. $0.1 \%$ chlorhexidine mouthwash for gingival inflammation in diabetic adults: double blind study. Journal de Parodontologie 1989;8(3):299-310.

\section{Yates 2002 \{published data only\}}

Yates R, Shearer BH, Huntington E, Addy M. A method to compare four mouthrinses: time to gingivitis level as the primary outcome variable. Journal of Clinical Periodontology 2002;29(6):519-23.

\section{References to studies awaiting assessment}

\section{CTRI/2011/05/001774 \{published data only\}}

CTRI/2011/05/001774. Non-inferiority efficacy study of 2 different formulations of $0.12 \%$ chlorhexidine gluconate oral rinse with (Oroclense) and without alcohol (Oroclear) for treatment of gingivitis. www.ctri.nic.in/Clinicaltrials/ pmaindet2.php?trialid=2390 (first received 31 May 2011).

NCT01898000 \{published data only\}

NCT01898000. 'Triphala' - A new herbal mouthwash in gingivitis: a randomized controlled clinical trial. clinicaltrials.gov/show/ NCT01898000 (first received 3 July 2013).

\section{NCT02065414 \{published data only\}}

NCT02065414. Four weeks clinical efficacy of an Ethyl Lauroyl Arginate $\mathrm{HCL}$ (LAE) mouthrinse: effect on gingivitis. clinicaltrials.gov/show/NCT02065414 (first received 16 February 2014).

\section{Radvar 2016 \{published data only\}}

Radvar M, Moeintaghavi A, Tafaghodi M, Ghanbari H, Fatemi K, Mokhtari MR, et al. Clinical efficacy of a herbal mouth wash composed of Salix alba, Malva sylvestrais and Althaea officinalis in chronic periodontitis patients. Journal of Herbal Medicine 2016;6(1):24-7.

\section{References to ongoing studies}

NCT02709785 \{published data only\}

NCT02709785. SmartMouth Advanced Clinical Formula clinical research design protocol. clinicaltrials.gov/show/NCT02709785 (first received 9 March 2016). 


\section{Additional references}

\section{Abdellatif 1987}

Abdellatif HM, Burt BA. An epidemiological investigation into the relative importance of age and oral hygiene status as determinants of periodontitis. Journal of Dental Research 1987;66(1):13-8.

\section{Addy 1985}

Addy M, Moran J. Extrinsic tooth discoloration by metals and chlorhexidine. 2. Clinical staining produced by chlorhexidine, iron and tea. British Dental Journal 1985;159(10):331-4.

\section{Addy 1985a}

Addy M, Moran J, Griffiths AA, Willswood NJ. Extrinsic tooth discoloration by metals and chlorhexidine. 1. Surface protein denaturation or dietary precipitation. British Dental Journal 1985;159(9):281-5.

\section{Addy 1986}

Addy M. Chlorhexidine compared with other locally delivered antimicrobials. A short review. Journal of Clinical Periodontology 1986;13(10):957-64.

\section{Addy 2005}

Addy M, Sharif N, Moran J. A non-staining chlorhexidine mouthwash? Probably not: a study in vitro. International Journal of Dental Hygiene 2005;3(2):59-63.

\section{Ainamo and Bay 1975}

Ainamo J, Bay I. Problems and proposals for recording gingivitis and plaque. International Dental Journal 1975;25(4):229-35.

\section{Al-Harthi 2013}

Al-Harthi LS, Cullinan MP, Leichter JW, Thomson WM. The impact of periodontitis on oral health-related quality of life: a review of the evidence from observational studies. Australian Dental Journal 2013;58(3):274-7.

\section{Axelsson 2004}

Axelsson P, Nystrom B, Lindhe J. The long-term effect of a plaque control program on tooth mortality, caries and periodontal disease in adults. Results after 30 years of maintenance. Journal of Clinical Periodontology 2004;31(9):749-57.

\section{Begg 1996}

Begg C, Cho M, Eastwood S, Horton R, Moher D, Olkin I, et al. Improving the quality of reporting of randomized controlled trials. The CONSORT statement. JAMA 1996;276(8):637-9.

\section{Berchier 2008}

Berchier CE, Slot DE, Haps S, Van der Weijden GA. The efficacy of dental floss in addition to a toothbrush on plaque and parameters of gingival inflammation: a systematic review. International Journal of Dental Hygiene 2008;6(4):265-79.

\section{Berchier 2010}

Berchier CE, Slot DE, Van der Weijden GA. The efficacy of $0.12 \%$ chlorhexidine mouthrinse compared with $0.2 \%$ on plaque accumulation and periodontal parameters: a systematic review. Journal of Clinical Periodontology 2010;37(9):829-39.

\section{Björby and Löe 1966}

Bjorby A, Löe $H$. The relative significance of different local factors in the initiation and development of periodontal inflammation. Journal of Periodontal Research 1966;2:76-7.

\section{Bonesvoll 1974}

Bonesvoll P, Lokken P, Rolla G. Influence of concentration, time, temperature and $\mathrm{pH}$ on the retention of chlorhexidine in the human oral cavity after mouth rinses. Archives of Oral Biology 1974;19(11):1025-9.

\section{Bonesvoll 1974a}

Bonesvoll P, Lokken P, Rolla G, Paus PN. Retention of chlorhexidine in the human oral cavity after mouth rinses. Archives of Oral Biology 1974;19(3):209-12.

\section{Bonesvoll 1978}

Bonesvoll P, Gjermo P. A comparison between chlorhexidine and some quaternary ammonium compounds with regard to retention, salivary concentration and plaque-inhibiting effect in the human mouth after mouth rinses. Arch Oral Biol 1978;23(4):289-94. [0003-9969: (Print)]

\section{Boyle 2014}

Boyle P, Koechlin A, Autier P. Mouthwash use and the prevention of plaque, gingivitis and caries. Oral Disease 2014;20 Suppl 1:1-68.

\section{Caton and Polson 1985}

Caton JG, Polson AM. The interdental bleeding index: a simplified procedure for monitoring gingival health. Compendium of Continuing Education in Dentistry 1985;6(2):88, 90-2.

\section{Chapple 2015}

Chapple IL, van der Weijden F, Doerfer C, Herrera D, Shapira L, Polak D, et al. Primary prevention of periodontitis: managing gingivitis. Journal of Clinical Periodontology 2015;42 Suppl 16:S71-6.

\section{Ciancio 1986}

Ciancio SG. Current status of indices of gingivitis. Journal of Clinical Periodontology 1986;13(5):375-8, 381-2.

\section{Cowell 1975}

Cowell CR, Saxton CA, Sheiham A, Wagg BJ. Testing therapeutic measures for controlling gingivitis in man: A suggested protocol. Journal of Clinical Periodontology 1975;2:231-40.

\section{Cumming and Löe 1973}

Cumming BR, Löe H. Optimal dosage and method of delivering chlorhexidine solutions for the inhibition of dental plaque. Journal of Periodontal Research 1973;8(2):57-62.

\section{Cunha-Cruz 2007}

Cunha-Cruz J, Hujoel PP, Kressin NR. Oral health-related quality of life of periodontal patients. Journal of Periodontal Research 2007;42(2):169-76. 


\section{de la Rosa and Sturzenberger 1976}

De la Rosa M, Sturzenberger OP. Clinical reduction of gingivitis through the use of a mouthwash containing two quaternary ammonium compounds. Journal of Periodontology 1976;47(9):535-7.

\section{DePaola 2007}

DePaola LG, Spolarich AE. Safety and efficacy of antimicrobial mouthrinses in clinical practice. Journal of Dental Hygiene 2007;81(5):1-16.

\section{Egger 1997}

Egger M, Davey Smith G, Schneider M, Minder C. Bias in meta-analysis detected by a simple, graphical test. $B M J$ 1997;315(7109):629-34.

\section{Eley 1999}

Eley BM. Antibacterial agents in the control of supragingival plaque - a review. British Dental Journal 1999;186(6):286-96.

\section{Emilson 1977}

Emilson CG. Susceptibility of various microorganisms to chlorhexidine. Scandinavian Journal of Dental Research 1977;85(4):255-65.

\section{Ennever 1961}

Ennever J, Sturzenberger OP, Radike AW. The calculus surface index method for scoring clinical calculus studies. Journal of Periodontology 1961;32:54-7.

\section{Flotra 1971}

Flotra L, Gjermo P, Rolla G, Waerhaug J. Side effects of chlorhexidine mouth washes. Scandinavian Journal of Dental Research 1971;79(2):119-25.

\section{Foulkes 1973}

Foulkes DM. Some toxicological observations on chlorhexidine. Journal of Periodontal Research Supplement 1973;12:55-60.

\section{GRADE 2004}

GRADE Working Group. Grading quality of evidence and strength of recommendations. BMJ 2004;328(7454):1490.

\section{Greene and Vermillion 1964}

Greene JC, Vermillion JR. The Simplified Oral Hygiene Index. Journal of the American Dental Association 1964;68:7-13.

\section{Greenstein 1986}

Greenstein G, Berman C, Jaffin R. Chlorhexidine. An adjunct to periodontal therapy. Journal of Periodontology 1986;57(6):370-7.

\section{Grundemann 2000}

Grundemann LJ, Timmerman MF, ljzerman Y, van der Velden U, van der Weijden GA. Stain, plaque and gingivitis reduction by combining chlorhexidine and peroxyborate. Journal of Clinical Periodontology 2000;27(1):9-15.

\section{Gunsolley 2006}

Gunsolley JC. A meta-analysis of six-month studies of antiplaque and antigingivitis agents. Journal of the American Dental Association 2006;137(12):1649-57.

\section{Gunsolley 2010}

Gunsolley JC. Clinical efficacy of antimicrobial mouthrinses. Journal of Dentistry 2010;38 Suppl 1:S6-10.

\section{Higgins 2011}

Higgins JP, Green S, editor(s). Cochrane Handbook for Systematic Reviews of Interventions Version 5.1.0 (updated March 2011). The Cochrane Collaboration, 2011. Available from handbook.cochrane.org.

\section{Hull 1980}

Hull PS. Chemical inhibition of plaque. Journal of Clinical Periodontology 1980;7(6):431-42.

\section{Jenkins 1988}

Jenkins S, Addy M, Wade W. The mechanism of action of chlorhexidine. A study of plaque growth on enamel inserts in vivo. Journal of Clinical Periodontology 1988;15(7):415-24.

\section{Jones 1997}

Jones CG. Chlorhexidine: is it still the gold standard? Periodontology 2000 1997;15:55-62.

\section{Karpinski 2015}

Karpinski TM, Szkaradkiewicz AK. Chlorhexidine - pharmacobiological activity and application. European Review for Medical and Pharmacological Sciences 2015;19(7):1321-6.

\section{Kassebaum 2014}

Kassebaum NJ, Bernabé E, Dahiya M, Bhandari B, Murray CJ, Marcenes W. Global burden of severe periodontitis in 1990-2010: a systematic review and meta-regression. Journal of Dental Research 2014;93(11):1045-53.

\section{Keijser 2003}

Keijser JAM, Verkade H, Timmerman MF, van der Weijden FA. Comparison of 2 commercially available chlorhexidine mouthrinses. Journal of Periodontology 2003;74(2):214-8.

\section{Kornman 1986}

Kornman KC. The role of supragingival plaque in the prevention and treatment of periodontal diseases. Journal of Periodontal Research 1986;21:5-22.

\section{Lang 2009}

Lang NP, Schätzle MA, Löe H. Gingivitis as a risk factor in periodontal disease. Journal of Clinical Periodontology 2009;36 Suppl 10:3-8.

\section{Lang and Räber 1981}

Lang NP, Räber K. Use of oral irrigators as vehicle for the application of antimicrobial agents in chemical plaque control. Journal of Clinical Periodontology 1981;8(3):177-88. 


\section{Lange 1977}

Lange DE, Plagmann HC, Eenboom A, Promesberger A. Clinical methods for the objective evaluation of oral hygiene. Deutsche Zahnarztliche Zeitschrift 1977;32(1):44-7.

\section{Lefebvre 2011}

Lefebvre C, Manheimer E, Glanville J. Chapter 6: Searching for studies. In: Higgins PJ, Green S, editor(s). Cochrane Handbook for Systematic Reviews of Interventions Version 5.1.0 (updated March 2011). The Cochrane Collaboration, 2011. Available from handbook.cochrane.org.

\section{Lobene 1968}

Lobene RR. Effect of dentifrices on tooth stains with controlled brushing. Journal of the American Dental Association 1968;77(4):849-55.

\section{Lobene 1982}

Lobene RR, Soparkar PM, Newman MB. Use of dental floss. Effect on plaque and gingivitis. Clinical Preventive Dentistry 1982;4(1):5-8.

\section{Lobene 1986}

Lobene RR, Weatherford T, Ross NM, Lamm RA, Menaker L. A modified gingival index for use in clinical trials. Clinical Preventive Dentistry 1986;8(1):3-6.

\section{Löe 1965}

Löe $H$, Theilade E, Jensen SB. Experimental gingivitis in man. Journal of Periodontology 1965;36:177-87.

\section{Löe 1967}

Löe H. The Gingival Index, the Plaque Index and the Retention Index Systems. Journal of Periodontology 1967;38(6):Suppl:610-6.

\section{Löe 1970}

Löe H, Schiott CR. The effect of mouthrinses and topical application of chlorhexidine on the development of dental plaque and gingivitis in man. Journal of Periodontal Research 1970;5(2):79-83.

\section{Löe and Silness 1963}

Löe H, Silness J. Periodontal disease in pregnancy. I. Prevalence and severity. Acta Odontologica Scandinavica 1963;21:533-51.

\section{Loesche 1979}

Loesche WJ. Clinical and microbiological aspects of chemotherapeutic agents used according to the specific plaque hypothesis. Journal of Dental Research 1979;58(12):2404-12.

\section{Lorenz 2009}

Lorenz K, Bruhn G, Netuschil L, Heumann C, Hoffmann T. How to select study designs and parameters to investigate the effect of mouthrinses? Part I: rationale and background. Journal of Physiology and Pharmacology 2009;60 Suppl 8:77-83.

\section{Mandel 1994}

Mandel ID. Antimicrobial mouthrinses: overview and update. Journal of the American Dental Association 1994;125 Suppl 2:2S-10S

\section{Manhold 1965}

Manhold JH, Volpe AR, Parker L, Adams SH. In vivo calculus assessment. II. A comparison of scoring techniques. Journal of Periodontology 1965;36:299-304.

\section{Marcenes 2013}

Marcenes W, Kassebaum NJ, Bernabe E, Flaxman A, Naghavi M, Lopez A, et al. Global burden of oral conditions in 1990-2010: a systematic analysis. Journal of Dental Research 2013;92(7):592-7.

\section{Marinone 2000}

Marinone MG, Savoldi E. Chlorhexidine and taste. Influence of mouthwashes concentration and of rinsing time. Minerva Stomatologica 2000;49(5):221-6.

\section{Marsh 1992}

Marsh PD. Microbiological aspects of the chemical control of plaque and gingivitis. Journal of Dental Research 1992;71(7):1431-8.

\section{Moher 2001}

Moher D, Schulz KF, Altman DG. The CONSORT statement: revised recommendations for improving the quality of reports of parallel-group randomised trials. Lancet 2001;357(9263):1191-4.

\section{Moran 2008}

Moran JM. Home-use oral hygiene products: mouthrinses. Periodontology 2000 2008;48:42-53.

\section{Muhlemann 1977}

Muhlemann HR. Psychological and chemical mediators of gingival health. Journal of Preventive Dentistry 1977;4(4):6-17.

\section{Needleman 2004}

Needleman I, McGrath C, Floyd P, Biddle A. Impact of oral health on the life quality of periodontal patients. Journal of Clinical Periodontology 2004;31(6):454-7.

\section{Newcombe 1995}

Newcombe RG, Addy M, Mckeown S. Residual effect of chlorhexidine gluconate in 4-day plaque regrowth crossover trials, and its implications for study design. Journal of Periodontal Research 1995;30(5):319-24.

\section{Ng 2006}

$\mathrm{Ng}$ SK, Leung WK. Oral health-related quality of life and periodontal status. Community Dentistry and Oral Epidemiology 2006;34(2):114-22.

\section{NICE 2012}

NICE. National Institute for Health and Care Excellence (NICE). NICE Clinical Knowledge Summaries. Gingivitis and Periodontitis, 2012. cks.nice.org.uk/gingivitis-andperiodontitis\#!backgroundsub:10 (accessed March 2015).

\section{O'Leary 1972}

O'Leary TJ, Drake RB, Naylor JE. The plaque control record. Journal of Periodontology 1972;43(1):38. 


\section{Ohrn 2009}

Ohrn K, Sanz M. Prevention and therapeutic approaches to gingival inflammation. Journal of Clinical Periodontology 2009;36 Suppl 10:20-6.

\section{Pemberton 2012}

Pemberton MN, Gibson J. Chlorhexidine and hypersensitivity reactions in dentistry. British Dental Journal 2012;213(11):547-50.

\section{Polson and Caton 1985}

Polson AM, Caton JG. Current status of bleeding in the diagnosis of periodontal diseases. Journal of Periodontology 1985;56(11 Suppl):1-3.

\section{Puig Silla 2008}

Puig Silla M, Montiel Company JM, Almerich Silla JM. Use of chlorhexidine varnishes in preventing and treating periodontal disease. A review of the literature. Medicina Oral, Patología Oral y Cirugía Bucal 2008;13(4):E257-60.

\section{Quigley 1962}

Quigley GA. Comparative cleansing efficiency of manual and power brushing. Journal of the American Dental Association 1962;65(1):26-9.

\section{Rolla 1971}

Rolla G, Loe H, Schiott CR. Retention of chlorhexidine in the human oral cavity. Archives of Oral Biology 1971;16(9):1109-16.

\section{Salzer 2015}

Salzer S, Slot DE, Van der Weijden FA, Dorfer CE. Efficacy of inter-dental mechanical plaque control in managing gingivitis a meta-review. Journal of Clinical Periodontology 2015;42:S92S105.

\section{Saxer 1975}

Saxer UP, Mühlemann HR. Motivation and education. SSO Schweizerische Monatsschrift fur Zahnheilkunde 1975;85(9):905-19.

\section{Saxton and van der Ouderaa 1989}

Saxton CA, van der Ouderaa FJG. The effect of a dentifrice containing zinc citrate and triclosan on developing gingivitis. Journal of Periodontal Research 1989;24(1):75-80.

\section{Schatzle 2004}

Schatzle M, Loe H, Lang NP, Burgin W, Anerud A, Boysen H. The clinical course of chronic periodontitis. Journal of Clinical Periodontology 2004;31(12):1122-7.

\section{Schulz 2010}

Schulz KF, Altman DG, Moher D, Group C. CONSORT 2010 Statement: Updated guidelines for reporting parallel group randomised trials. Journal of Clinical Epidemiology 2010;63(8):834-40.

\section{Serrano 2015}

Serrano J, Escribano M, Roldán S, Martín C, Herrera D. Efficacy of adjunctive anti-plaque chemical agents in managing gingivitis: a systematic review and meta-analysis. Journal of Clinical Periodontology 2015;42(16):S106-38.

\section{Shaw 1977}

Shaw L, Murray JJ. New index for measuring extrinsic stain in clinical trials. Community Dentistry and Oral Epidemiology 1977;5(3):116-20.

\section{Silness and Löe 1964}

Silness J, Löe H. Periodontal disease in pregnancy. II. Correlation between oral hygiene and periodontal condition. Acta Odontologica Scandinavica 1964;22:121-35.

\section{Slot 2012}

Slot DE, Wiggelinkhuizen L, Rosema NA, van der Weijden GA. The efficacy of manual toothbrushes following a brushing exercise: a systematic review. International Journal of Dental Hygiene 2012;10(3):187-97.

\section{Stata 2015 [Computer program]}

Stata Data Analysis and Statistical Software: release 14. StataCorp LP, 2015.

\section{Tomás 2010}

Tomás I, Cousido MC, Garcia-Caballero L, Rubido S, Limeres J, Diz P. Substantivity of a single chlorhexidine mouthwash on salivary flora: Influence of intrinsic and extrinsic factors. Journal of Dentistry 2010;38(7):541-6.

\section{Tonetti 2015}

Tonetti MS, Chapple ILC, Jepsen S, Sanz M. Primary and secondary prevention of periodontal and peri-implant diseases - Introduction to, and objectives of the 11th European workshop on periodontology consensus conference. Journal of Clinical Periodontology 2015;42(16):S1-S4.

\section{Turesky 1970}

Turesky S, Gilmore ND, Glickman I. Reduced plaque formation by the chloromethyl analogue of victamin C. Journal of Periodontology 1970;41(1):41-3.

\section{van der Weijden 1994}

van der Weijden GA, Timmerman MF, Nijboer A, Reijerse E, van der Velden U. Comparison of different approaches to assess bleeding on probing as indicators of gingivitis. Journal of Clinical Periodontology 1994;21(9):589-94.

\section{van der Weijden 2005}

van der Weijden GA, Hioe KP. A systematic review of the effectiveness of self-performed mechanical plaque removal in adults with gingivitis using a manual toothbrush. Journal of Clinical Periodontology 2005;32:214-28.

\section{van der Weijden 2010}

van der Weijden GA, ten Heggeler JMAG, Slot DE, Rosema NAM, van der Velden U. Parotid gland swelling following mouthrinse use. International Journal of Dental Hygiene 2010;8(4):276-9. 


\section{van der Weijden 2011}

van der Weijden F, Slot DE. Oral hygiene in the prevention of periodontal diseases: the evidence. Periodontology 2000 2011;55(1):104-23.

\section{van der Weijden 2015}

van der Weijden FA, van der Sluijs E, Ciancio SG, Slot DE. Can chemical mouthwash agents achieve plaque/gingivitis control? Dental Clinics of North America 2015;59(4):799-829.

\section{Van Strydonck 2012}

Van Strydonck DA, Slot DE, van der Velden U, van der Weijden F. Effect of a chlorhexidine mouthrinse on plaque, gingival inflammation and staining in gingivitis patients: a systematic review. Journal of Clinical Periodontology 2012;39(11):1042-55.

\section{Volpe 1965}

Volpe AR, Manhold JH, Hazen SP. In vivo calculus assessment. I. A method and its examiner reproducibility. Journal of Periodontology 1965;36:292-8.

\section{CHARACTERISTICS OF STUDIES}

Characteristics of included studies [ordered by study ID]

\section{Watts 2001}

Watts A, Addy M. Tooth discolouration and staining: a review of the literature. British Dental Journal 2001;190(6):309-16.

\section{Zimmer 2005}

Zimmer S, Strauss J, Bizhang M, Krage T, Raab WHM, Barthel C. Efficacy of the Cybersonic in comparison with the Braun 3D Excel and a manual toothbrush. Journal of Clinical Periodontology 2005;32(4):360-3.

\section{References to other published versions of this review}

\section{James 2010}

James P, Parnell C, Harding M, Whelton $\mathrm{H}$, Worthington HV, Beirne PV. Chlorhexidine mouthrinse as an adjunctive treatment for gingival health. Cochrane Database of Systematic Reviews 2010, Issue 9. Art. No: CD008676. [DOI: 10.1002/14651858.CD008676]

* Indicates the major publication for the study

Anauate-Netto 2014

Study characteristics

\begin{tabular}{|c|c|}
\hline \multirow[t]{4}{*}{ Methods } & Trial design: parallel ( 3 arms - 2 arms relevant to this review) \\
\hline & Location: dental clinics at University Bandeirante of Sao Paulo, Sao Paulo, Brazil \\
\hline & Number of centres: 1 \\
\hline & $\begin{array}{l}\text { Study duration: the recruitment period is not stated. Study duration and duration of rinsing was } 4 \\
\text { weeks }\end{array}$ \\
\hline \multirow[t]{8}{*}{ Participants } & Participants: healthy adults \\
\hline & $\begin{array}{l}\text { Inclusion criteria: at least } 20 \text { teeth, no clinical signs of periodontal disease, aged between } 18 \text { and } 55 \\
\text { years, normal saliva secretion rate }\end{array}$ \\
\hline & Exclusion criteria: smokers, pregnancy, taking oral topical or systemic medication \\
\hline & $\begin{array}{l}\text { Baseline gingivitis: (Papillary Bleeding Score) overall (3 groups) mean 1. Gp A: mean 1.1 (SD 0.5); Gp B: } \\
\text { mean 1.0 (SD 0.5); Gp C: mean 0.9 (SD 0.4) }\end{array}$ \\
\hline & $\begin{array}{l}\text { Age at baseline (years): overall: 40. Gp A: mean } 41.6 \text { (SD 13.4); Gp B: mean } 39.4 \text { (SD 9.8); Gp C: mean } 39 \\
\text { (SD 11.7) }\end{array}$ \\
\hline & $\begin{array}{l}\text { Gender: overall: male } 24 \text { (40\%), female } 36(60 \%) \text {. Gp A: male } 7 \text { (35\%), female } 13(65 \%) \text {; Gp B: male } 8 \\
(40 \%) \text {, female } 12(60 \%) \text {; Gp C: male } 9(45 \%) \text {, female } 11(55 \%)\end{array}$ \\
\hline & Number randomised: 60 (Gp A: 20; Gp B: 20; Gp C: 20) \\
\hline & Number evaluated: 60 (Gp A: 20; Gp B: 20; Gp C: 20) \\
\hline
\end{tabular}

Interventions

Comparison: $\mathrm{CHX}$ rinse versus propolis rinse* versus placebo rinse

Gp A ( $n=20)$ : CHX 0.12\%, $15 \mathrm{~mL}$, twice daily, duration not specified, 4 weeks 
Anauate-Netto 2014 (Continued)

${ }^{*} \mathrm{Gp} \mathrm{B}(\mathrm{n}=20)$ : alcohol-free, $2 \%$ typified propolis (plus mint flavour, polioxyethelers, sorbitol, blue colour and water), $15 \mathrm{~mL}$, twice daily, duration not specified, 4 weeks. We excluded this arm from the risk of bias and analysis

Gp C ( $n=20)$ : placebo (same ingredients as Gp B without the propolis), $15 \mathrm{~mL}$, twice daily, duration not specified, 4 weeks

Prophylaxis at baseline: not reported

$\mathrm{OHI}$ : none given. Subjects followed their usual oral hygiene procedures

Non-supervised rinsing

Timing of mouthrinsing in relation to toothbrushing: quote: "Rinsing was performed in the morning and before bedtime after ordinary oral hygiene procedures"

Outcomes

Gingivitis (gingivial inflammation: Papillary Bleeding Score, gingival bleeding: $P B S \geq 2$ ); adverse reactions; assessed at 4 weeks

Funding Quote: "This study was supported by a grant from FAPESP (Fundacao de amparo a pesquisado estado de Sao Paulo) protocol no. 2007/53047-3"

Notes

Sample size calculation: quote: "It was not possible to conduct a sample size and power analysis... because of lack of randomized clinical trials of propolis on gingivitis"

Adverse effects: $\mathrm{Gp} \mathrm{A}(\mathrm{CHX}): 23$ reports (burning sensation, taste alteration, yellow teeth, breath alteration, tongue burning, mucosal irritation, bitter taste); Gp B (propolis): 7 reports (breath alteration, burning sensation, taste alteration, yellow teeth, bitter taste); Gp C (placebo): 9 reports (taste alteration)

Non-smokers

Declarations/conflicts of interest: none stated

\section{Risk of bias}

Bias Authors' judgement Support for judgement

Random sequence genera- Low risk $\quad$ Quote: "a computer-generated list of random numbers was used"
tion (selection bias)

Comment: adequate method of random sequence generation

Allocation concealment Low risk

(selection bias)
Quote: "Rinses were prepared in dark-bottles, which were consecutively numbered according to the randomization schedule... Study coordinator, examiners, and participants were unaware of group allocation. The group identity was generated and kept in Florianopolis, SC, Brazil while the study was conducted in Sao Paulo, SP, Brazil"

Comment: remote/central randomisation should have ensured that the random sequence was implemented as it was generated, without any manipulation

\section{Blinding of participants High risk} and personnel (perfor-

mance bias)

All outcomes

\section{Quote: "double-blind placebo-controlled" and "rinses}

were prepared in dark-bottles." The placebo mouthrinse matched the propolis mouthrinse without the active ingredient

Comment: the level of adverse reactions in the $\mathrm{CHX}$ group $(\mathrm{n}=23)$ was higher than in the propolis $(n=7)$ and placebo groups $(n=9)$ may have meant that participants could have worked out which group they were in and this could have affected their oral health behaviours and hence the outcome.The direction of this potential bias is not clear 
Anauate-Netto 2014 (Continued)

Blinding of outcome as- High risk Quote: "double-blind". The incidence of adverse reactions in the CHX group (n sessment (detection bias) $=23)$ was higher than in the propolis $(n=7)$ and placebo groups $(n=9)$

All outcomes

Comment: the authors do not report precisely who was blinded, but even if this includes the outcome assessors the higher level of adverse effects including tooth staining in the $\mathrm{CHX}$ group could have allowed the outcome assessors to work out which individuals were in the $\mathrm{CHX}$ group and could have affected the outcome assessment. The direction of this potential bias is not clear

Incomplete outcome data Low risk All randomised participants were included in the analyses
(attrition bias)

All outcomes

Selective reporting (re- High risk
porting bias)
porting bias)

All outcomes stated in the methods section are reported in the results section. Adverse effects including 'yellow teeth' are reported aggregated as the total number of participants affected by group. Insufficient information on tooth staining is reported for us to use the data in a meta-analysis

Other bias Low risk

Quote: "PBS measurements were performed by an experienced examiner (AAA). Intra-examiner reliability exercises revealed a Kappa test score of 0.85 , indicating adequate reproducibility of PBS measurements"

Comment: the potential for differential diagnostic activity was minimised.

Groups were balanced at baseline (NS differences) for age, gender and race. The groups appear balanced for PBS at baseline

\section{Anderson 1997}

\section{Study characteristics}

\begin{tabular}{ll}
\hline Methods & Trial design: parallel (2 arms) \\
Location: Postgraduate Clinic of Orthodontics, University of Texas-Houston, USA \\
Number of centres: 1 \\
Study duration: recruitment period is not reported. Study duration and duration of rinsing was 3 \\
months \\
Participants: adolescents undergoing orthodontic treatment \\
Inclusion criteria: quote: "The participants did not suffer from any systemic complications nor were \\
they taking any medications that may have had an effect on the oral tissues. All the participants had \\
banding of at least one molar per quadrant" \\
Exclusion criteria: not reported \\
Baseline gingivitis: (GI) overall 0.925. Gp A: mean 1.04 (no SD); Gp B: mean 0.81 (no SD) \\
Age at baseline (years): range 11-15 \\
Gender: not reported \\
Number randomised: 32 (Gp A: 16; Gp B: 16) \\
Number evaluated: 28 (Gp A: 13; Gp B: 15) \\
Fants
\end{tabular}

Interventions

Comparison: $\mathrm{CHX}$ rinse versus placebo rinse 
Gp B ( $n=16)$ : placebo (identical to the experimental rinse except for the active ingredient), $15 \mathrm{~mL}$, twice daily, 30 seconds, 3 months

Prophylaxis at baseline: quote: "they received a dental prophylaxis which included the removal of plaque, calculus and stains from the teeth by scaling and polishing" (8-10 days before baseline measurements)

OHI: they received oral and written toothbrushing instructions with the same toothpaste (Crest regular flavour) to be performed twice daily. The method used was the Modified Bass technique. Soft rounded toothbrushes were supplied to all patients

Non-supervised rinsing

Timing of mouthrinsing in relation to toothbrushing: not reported

Postrinse instructions: not reported

$\begin{array}{ll}\text { Outcomes } & \text { Gingivitis (gingival inflammation: GI Löe \& Silness 1963); plaque (PI Silness \& Löe 1964); calculus (Reten } \\ \text { tion Index, Löe 1967); assessed at 1, } 2 \text { and } 3 \text { months follow-up } \\ \text { Probing depths (CP-12 Hu Friedy periodontal probe) - not relevant to this review and tooth staining } \\ \text { (Discolouration Index, Lang 1981); assessed at } 3 \text { months only }\end{array}$

Funding

Quote: "Partially supported by Procter \& Gamble, by providing the chlorhexidine and placebo for the patients in this study"

Sample size calculation: not reported
Adverse effects: quote: "...no side effects were reported as a result of the use of either the experimental
or the control solutions except for one patient in CHX group who acknowledged a burning sensation on
the mucosa after the use of the experimental solution." No significant differences were found between
the groups for discolouration or calculus accumulation, although tooth discolouration "was more ev-
ident in the experimental group" and for calculus accumulation "the higher mean values were always
found in the experimental group"
Declarations/conflicts of interest: none stated

\section{Risk of bias}

\begin{tabular}{|c|c|c|}
\hline Bias & Authors' judgement & Support for judgement \\
\hline $\begin{array}{l}\text { Random sequence genera- } \\
\text { tion (selection bias) }\end{array}$ & Unclear risk & $\begin{array}{l}\text { Quote: "They were randomised to two groups" } \\
\text { Comment: no description of the method of random sequence generation }\end{array}$ \\
\hline $\begin{array}{l}\text { Allocation concealment } \\
\text { (selection bias) }\end{array}$ & Unclear risk & No information is provided \\
\hline $\begin{array}{l}\text { Blinding of participants } \\
\text { and personnel (perfor- } \\
\text { mance bias) } \\
\text { All outcomes }\end{array}$ & High risk & $\begin{array}{l}\text { Quote: "The control group received exactly the same instructions with a } \\
\text { flavoured placebo solution which was identical to the experimental solution } \\
\text { except for the active ingredient..." and "..the discoloration was more evident } \\
\text { in the experimental group but it was not statistically significant" } \\
\text { Comment: the study is not described as blind by the authors but the meth- } \\
\text { ods described indicate that it was intended for the participants to be unaware } \\
\text { of the allocated intervention. However, the higher level of staining in the ac- } \\
\text { tive group (although not statistically significant) meant that participants could } \\
\text { have worked out which group they were in and this could have affected their }\end{array}$ \\
\hline
\end{tabular}




Blinding of outcome as-
sessment (detection bias)
All outcomes

All outcomes

\section{No information is provided}

Comment: the study is not described as blind by the authors. The higher level of staining in the $\mathrm{CHX}$ group (although not statistically significant) meant that the outcome assessor could not be adequately blinded and this could have affected the outcome assessment. The direction of this potential bias is unclear

Incomplete outcome data High risk
(attrition bias)

$12.5 \%(4 / 32)$ of randomised participants were not included in the analysis. Attrition by group: $\mathrm{CHX} 3 / 16(18.8 \%)$, placebo $1 / 16(6.3 \%)$

2 individuals left the study after they received the baseline prophylaxis $(\mathrm{CHX}$ group), 1 before the 30 day evaluation (CHX group) and 1 was unable to return for the 60 and 90 day evaluations (control group)

Comment: attrition was quite high in the $\mathrm{CHX}$ group as proportion of the total number in the group. Although the attrition is unlikely to be due to the intervention ( $\mathrm{CHX)}$ ( 2 of the 3 participants who left the $\mathrm{CHX}$ group left the study before they started rinsing), if the missing participants had higher mean gingivitis scores in 1 group than the other, as the attrition rate increased, so would over/ understatement of the mean difference

$\begin{array}{ll}\begin{array}{l}\text { Selective reporting (re- } \\ \text { porting bias) }\end{array} & \text { High risk } \\ & \begin{array}{l}\text { Mean scores and standard deviations for all outcomes were reported by tooth } \\ \text { surface only which meant that with the exception of the tooth staining da- } \\ \text { ta (where we were able to combine data for buccal and lingual surfaces), we } \\ \text { could not use the data in meta-analyses }\end{array}\end{array}$

\begin{tabular}{ll}
\hline Other bias & $\begin{array}{l}\text { No information is provided on intra- or inter-examiner reproducibility. There } \\
\text { were no significant differences between the means of the plaque indices and } \\
\text { gingival indices recorded on all surfaces for both experimental and control } \\
\text { groups. The Retention Index, Discolouration Index and mean probing depth } \\
\text { measurements were also balanced } \\
\text { Mean age and gender are not reported }\end{array}$ \\
\hline
\end{tabular}

\section{Axelsson 1987}

\section{Study characteristics}

\begin{tabular}{|c|c|}
\hline \multirow[t]{4}{*}{ Methods } & Trial design: parallel ( 4 arms - 3 arms are relevant to this review) \\
\hline & Location: dental school, Sweden \\
\hline & Number of centres: 1 \\
\hline & Study duration: recruitment period is not reported. Study duration and duration of rinsing was 6 weeks \\
\hline \multirow{6}{*}{ Participants } & Inclusion criteria: not reported. \\
\hline & Exclusion criteria: periodontal attachment loss or bone loss \\
\hline & Baseline gingivitis (mean (SE): (GI Löe \& Silness 1963) overall 1.21. Gp A: mean 1.18 (0.08); Gp B: mean \\
\hline & 1.26 (0.07); Gp C: mean 1.19 (0.07); Gp D: mean $1.21(0.08))$ \\
\hline & Age at baseline (years): mean age not reported, range $16-50$ \\
\hline & Gender: not reported \\
\hline
\end{tabular}


Number evaluated: 88, (Gp A: 18; Gp B: 24, Gp C: 24; Gp D: 22)

${ }^{*} \mathrm{Gp} \mathrm{C}(\mathrm{n}=24)$ : Listerine, $20 \mathrm{~mL}$, twice daily, 30 seconds, 6 weeks. We excluded this arm from the risk of bias and analysis

Gp D ( $\mathrm{n}=24)$ : control rinse (composition not described), $20 \mathrm{~mL}$, twice daily, 30 seconds, 6 weeks

Prophylaxis at baseline: scaling and professional tooth cleaning after the baseline examination

$\mathrm{OHI}$ : they were supplied with a soft nylon toothbrush and Colgate MFP toothpaste, were instructed to refrain from using commercial mouthrinses and continued to exercise their regular non-supervised self-performed plaque control programs

Rinsing was supervised Mon-Fri and unsupervised at weekends

Timing of mouthrinsing in relation to toothbrushing is not reported

Outcomes Gingivitis (gingival inflammation: GI Löe \& Silness 1963, gingival bleeding: \% GI scores 0, 1, 2 or 3) as-
sessed at 6 weeks

Plaque (TQH Turesky 1970); extrinsic tooth stain (Lobene 1968); assessed at 3 and 6 weeks

A complete intraoral soft tissues examination was performed after 3 and 6 weeks

\begin{tabular}{ll}
\hline Funding & No information provided \\
\hline Notes & $\begin{array}{l}\text { Sample size calculation: quote: "The study was designed to provide a minimal power of } 0.70 \text { for detect- } \\
\text { ing a clinically important difference to be statistically significant at the } 0.05 \text { probability level. The final } \\
\text { sample size was based on the maximum determined among the separate requirements of the plaque } \\
\text { and gingival index scores." The sample size required is not reported }\end{array}$
\end{tabular}

Adverse effects: mucosal lesions occurred in 6 subjects in the Hibitane group (0.2\%), 1 subject in the control group complained of oral irritation which worsened during the study

The order of examinations were a) soft tissue condition, b) stain index, c) gingival index, d) plaque index

Declarations/conflicts of interest: none stated

Groups A and B were combined for the main analysis

\section{Risk of bias}

Bias Authors' judgement Support for judgement

Random sequence genera- Unclear risk tion (selection bias)
Quote: "Volunteers were assigned either to 1 of 3 different treatment groups or to a control group according to a randomised code by which double-blinding was maintained"

Comment: no description of the method of random sequence generation
Quote: "Volunteers were assigned either to 1 of 3 different treatment groups or to a control group according to a randomised code by which double-blinding was maintained" 
Comment: it is not clear who assigned the participants to the groups or whether participants or investigators could foresee assignment

Blinding of participants High risk
and personnel (perfor-
mance bias)
All outcomes

The study is described as double-blind. The authors do not report precisely who was blinded. The composition of the control is not described. There were negligible differences in extrinsic tooth staining between the groups at 3 and 6 weeks

Comment: neither participants nor personnel could be truly blind as different volumes and rinse times were used in different groups and rinsing was supervised on weekdays. Knowledge of which product was which could have influenced behaviour of participants and hence the outcome. The direction of this potential bias is not clear

\begin{tabular}{|c|c|c|}
\hline $\begin{array}{l}\text { Blinding of outcome as- } \\
\text { sessment (detection bias) } \\
\text { All outcomes }\end{array}$ & Low risk & $\begin{array}{l}\text { The authors state that the study is "double-blind" but do not report precise- } \\
\text { ly who was blinded. There were negligible differences in extrinsic tooth stain- } \\
\text { ing between the groups at } 3 \text { and } 6 \text { weeks. All intraoral examinations (including } \\
\text { tooth staining) were performed by a single examiner }\end{array}$ \\
\hline
\end{tabular}

Incomplete outcome data High risk
(attrition bias)

$11 \%(8 / 72)$ of randomised participants were not included in the analysis All outcomes Attrition by group: Hibitane $0.2 \% 6 / 24$ (25\%); Hibitane $0.1 \%$ (0\%); control $2 / 24$ $(8.3 \%)$. Reasons for attrition: Hibitane $0.2 \%$ : mucosal lesions; control: oral irritation, personal reasons

Comment: there were differential group losses with proportionately more losses from the Hibitane $0.2 \%$ group (due to the occurrence of mucosal lesions) which could have influenced the outcome Selective reporting (re- High risk
porting bias)
All outcomes stated in the methods section are reported in the results section. We calculated standard deviations for gingivitis and plaque from the standard errors reported in the tables. We also calculated the $\%$ of gingival units with a GI score of 2 or 3 however, no measure of variance was reported so we could not include the data in meta-analyses. Extrinsic tooth staining and adverse effects were adequately reported. We combined groups A and B for the main analysis

All intraoral examinations (including tooth staining) were performed by a single examiner. No information is provided on intraexaminer reproducibility. Mean gingivitis, plaque and stain scores at baseline appear to be reasonably balanced

The age range is quite wide (16-50) but baseline data on age and gender balance are not reported

\section{Study characteristics}

Trial design: parallel (3 arms - 2 arms are relevant to this review)
Location: Department of Pedodontics and Preventative Dentistry, Manipal College of Dental Sciences,
Manipal, India
Number of centres: 1
Study duration: recruitment period not reported. Duration of study is 9 months; duration of rinsing is
not explicitly stated, assumed to be 9 months


Bajaj 2011 (Continued)

Participants
Participants: quote: "Children with similar socioeconomic status, dietary habits, oral hygiene status, and KAP (knowledge, attitude and practice) status were included. Further, only children who had a minimum of one to two established carious lesions were considered. The subjects were selected from residential schools"

Exclusion criteria: not reported

Baseline gingivitis: GI Löe \& Silness 1967, Gp A (0.54 SD 0.22); Gp B (0.59 SD 0.73); Gp C (1.16 SD 0.21)

Age at baseline (years): 8 to 12 years, not reported by group

Gender: not reported

Number randomised: 1431 (not reported by group)

Number evaluated: 1309 (Gp A: 440; Gp B: 457; Gp C: 412)

\section{Comparison: $\mathrm{CHX}$ rinse versus Triphala rinse* versus control rinse}

Gp A (440 analysed): CHX (Clohex $0.1 \%$ (diluted from $0.2 \%)$ ) $10 \mathrm{~mL}, 1 \mathrm{~min}$, once daily

${ }^{\star}$ Gp B (457 analysed): Triphala (0.6\%) $10 \mathrm{~mL}$, not reported, once daily. We excluded this arm from the risk of bias and analysis

Gp C (412 analysed): control (distilled water) $10 \mathrm{~mL}$, not reported, once daily

[Conflicting reports for duration of rinsing - 2 min rinse reported in methods for $\mathrm{CHX}$ rinse (duration of rinsing for Triphala and control not reported) and $1 \mathrm{~min}$ rinse reported in discussion for all 3 rinses. Rinse duration stated in the discussion is reported here]

Prophylaxis at baseline: not reported

OHI: not reported

Rinsing supervised during the week, non-supervised at weekends and during vacations

Timing of mouthrinsing in relation to toothbrushing: not reported

Postrinsing instructions: quote: "The children were advised not to eat or rinse for the next $30 \mathrm{~min}$ "

Outcomes Gingivitis (gingival inflammation: GI Löe \& Silness 1967) and plaque (PI Silness \& Löe 1964) recorded at 3,6 and 9 months follow-up. Microbiological analysis (Streptococcus and Lactobacillus counts) at baseline 6 and 9 months follow-up (not relevant to this review)

\begin{tabular}{|c|c|}
\hline Funding & Quote: "This research project was funded by the Indian Council of Medical Research" \\
\hline \multirow[t]{3}{*}{ Notes } & $\begin{array}{l}\text { Sample size calculation: quote: "Large sample size was selected anticipating the possible attrition of } \\
\text { the sample due to varying cultural background and migration of few students to other schools" (actual } \\
\text { calculation not mentioned) }\end{array}$ \\
\hline & Adverse effects: not reported \\
\hline & Declarations/conflicts of interest: not reported \\
\hline
\end{tabular}

Risk of bias

Bias Authors' judgement Support for judgement

Random sequence genera- Unclear risk Quote: "the subjects were allocated to the specific treatment by block rantion (selection bias) domization"

Comment: no description of the method of random sequence generation 
Bajaj 2011 (Continued)

Allocation concealment Unclear risk No information is provided
(selection bias)

Blinding of participants High risk and personnel (performance bias)

All outcomes
Quotes: "it was a double-blind clinical trial.... Both solutions (chlorhexidine and Triphala) were made of identical colors. The bottles were then coded and then at the end of the study, the decoding was done" and "The schools were distributed in such a manner so that there was no intermingling within the students of different groups." The control solution was distilled water. Rinsing was supervised by teachers on school days

Comments: the authors do not report precisely who was blinded. Blinding of the teachers is not explicitly stated. Higher levels of tooth staining are likely to have been noticeable in the $\mathrm{CHX}$ group, however, tooth staining is not reported and therefore not enough information is provided upon which to base a judgement on the likely effect of staining. The control group rinsed with distilled water and could have worked out which group they were in which could have affected their oral health behaviours

$\begin{array}{lll}\begin{array}{l}\text { Blinding of outcome as- } \\ \text { sessment (detection bias) }\end{array} & \text { Unclear risk } & \begin{array}{l}\text { Quote: "it was a double-blind clinical trial..." No other information relating to } \\ \text { blinding of outcome assessment is reported }\end{array}\end{array}$
All outcomes blinding of outcome assessment is reported

Comment: the authors do not report precisely who was blinded. Higher levels of tooth staining are likely to have been noticeable in the $\mathrm{CHX}$ groups, however, tooth staining is not reported and therefore not enough information is provided upon which to base a judgement
Incomplete outcome data Low risk (attrition bias)

All outcomes
Attrition after 9 months (all arms included) 122/1431 (8.53\%). Numbers lost in each group are not reported. Authors report \% lost by group as follows: $\mathrm{CHX}$ $7.36 \%$, control $10.62 \%$. Reasons for losses: quote: "the majority of times attrition occurred due to family reasons where the parents changed the school of their child"

Comments: attrition is reasonably low (highest in the control group), reasonably balanced across groups and reasons for losses are unlikely to be related to the interventions

\begin{tabular}{|c|c|c|}
\hline $\begin{array}{l}\text { Selective reporting (re- } \\
\text { porting bias) }\end{array}$ & High risk & $\begin{array}{l}\text { There are marked inconsistencies between data reported in text and data re- } \\
\text { ported in figure } 1 \text { in relation to mean plaque scores. Tooth staining and ad- } \\
\text { verse effects are not reported }\end{array}$ \\
\hline Other bias & High risk & $\begin{array}{l}\text { The number of examiners is not reported and there is no reference to training } \\
\text { or calibration. Children are reported to have had similar SES, dietary habits, } \\
\text { oral hygiene methods and KAP (knowledge, attitudes and practice) status. } \\
\text { Children had a minimum of } 1 \text { to } 2 \text { established carious lesions. The control } \\
\text { group (distilled water) had much higher mean plaque and gingivitis scores } \\
\text { than the CHX group at baseline: plaque: CHX 0.76, control 1.76; gingivitis: CHX } \\
0.54 \text {, control 1.16. (Data taken from the text rather than figures: see comment } \\
\text { above about inconsistencies between the text and the figures) }\end{array}$ \\
\hline
\end{tabular}

\section{Study characteristics}

\begin{tabular}{ll}
\hline Methods & Trial design: parallel, 2 arms \\
& Location: not specified, but assumed to be the University of Western Ontario Dental School, Ontario, \\
Canada
\end{tabular}


Number of centres: assumed 1

Study duration: recruitment period not stated. Duration of study and duration of rinsing was 24 months

\section{Participants}

Participants: employees from 3 hospitals, from the University of Western Ontario, and from the general public of London, Ontario

Inclusion criteria: quote: "subjects had to be at least 18 years of age and with at least 16 natural teeth (including four molars) and some gingivitis"

Exclusion criteria: quote: "Persons with advanced periodontal disease and/or gross dental neglect, those taking antibiotics regularly or within one week of the baseline examination, and those with a history of hepatitis, tuberculosis, heart disease, or other severe or debilitating medical conditions were excluded"

Baseline gingivitis: not reported

Age at baseline: not reported but minimum age of 18 years

Gender: not reported

Number randomised: 456 (CHX 231, control 225)

Number evaluated at 6 months: 383 (CHX 190, control 193)

Number evaluated at 24 months: 272 (CHX 112, control 160)

\section{Interventions Comparison: $\mathrm{CHX}$ versus placebo rinse}

CHX 0.12\% ( $n=231): 15 \mathrm{~mL}$, twice daily, 30 seconds, 24 months

Control (placebo - composition not described) ( $\mathrm{n}=225): 15 \mathrm{~mL}$, twice daily, 30 seconds, 24 months

Prophylaxis at baseline: quote: "All subjects were then (after baseline assessment) given a professional scaling and prophylaxis by a dental hygienist." Professional prophylaxis and scaling was repeated for all individuals every 6 months

OHI: none. Quote: "Toothbrushes and Crest dentifrice were also made available, but no additional oral care instruction was provided"

Non-supervised rinsing

Timing of mouthrinsing in relation to toothbrushing and postrinse instructions are not reported

Outcomes Gingivitis (gingival inflammation: GI Löe \& Silness 1967, gingival bleeding: GI scores 2 or 3), at 6, 12, and 24 months

Plaque (TQH Turesky 1970), at 6, 12, and 24 months

Calculus (supragingival calculus deposits (Volpe-Manhold Calculus Index, Volpe 1965) and subgingival calculus deposits) at 24 months only

Adverse effects: quote: "Staining of the teeth was cited as the most common reason for subjects not continuing in the study." At the 24-month examination, subjects in the treatment group had higher mean supragingival calculus scores, however at the same time, more subjects in the CHX group were free of subgingival calculus. Soft tissue conditions were observed in $33 \%$ of the subjects at baseline, in $13 \%$ of the subjects at 1 year and in $12 \%$ of subjects at 2 years. The proportion and type of oral soft tissue conditions were similar for the treatment and control groups 


\section{Risk of bias}

Bias Authors' judgement Support for judgement

Random sequence genera- Unclear risk tion (selection bias)
Quote: "..subjects were stratified by gender, age, and periodontal status and then randomly assigned to a control or treatment. Subjects residing in the same household were assigned to the same treatment group"

Comment: no description of the method of random sequence generation

\begin{tabular}{ll}
\hline $\begin{array}{l}\text { Allocation concealment } \\
\text { (selection bias) }\end{array}$ & Unclear risk
\end{tabular}

$\begin{array}{ll}\begin{array}{l}\text { Blinding of participants } \\ \text { and personnel (perfor- }\end{array} & \text { High risk } \\ \begin{array}{l}\text { mance bias) } \\ \text { All outcomes }\end{array} & \begin{array}{l}\text { Tooth staining was noted in the CHX group } \\ \text { Comment: participant blinding is not mentioned but the use of a control } \\ \text { 'placebo' (composition not described) indicate that it was intended for the } \\ \text { participants to be unaware of the allocated intervention. However, the higher } \\ \text { level of staining in the CHX group meant that participants could have worked } \\ \text { out which group they were in and this could have affected their oral health } \\ \text { behaviours and hence the outcome. The direction of this potential bias is not } \\ \text { clear }\end{array}\end{array}$

\section{Blinding of outcome as- High risk sessment (detection bias)} All outcomes
Quote: "Toothcover devices and tongue-masking were used at the one-year gingivitis examination to eliminate potential examiner bias related to extrinsic staining." Tooth staining was noted in the $\mathrm{CHX}$ group

Comment: gingivitis and gingival bleeding results at 6 and 24 months and plaque and PPD results at 6,12 and 24 months are at high risk of bias due to the higher level of tooth staining in the $\mathrm{CHX}$ group. The outcome assessors could not be adequately blinded and this could have affected the outcome assessment. The direction of this potential bias is unclear. The impact of using tooth cover devices and tongue-masking at 12 months on blinding is not clear

\section{Incomplete outcome data High risk}

(attrition bias)

All outcomes

\section{6 months:}

$16 \%(73 / 456)$ of randomised participants were not included in the analysis at 6 months. Attrition by group CHX 41/231 (18\%), control 32/225 (14\%)

\section{4 months:}

$40 \%(184 / 456)$ of randomised participants were not included in the analysis at 24 months. Attrition by group CHX 119/231 (51.5\%), control 65/225 (28.9\%)

Reasons for attrition: tooth staining was the most common reason. Other reasons were not reported

Comment: attrition was high in both groups. There were proportionately more losses from the $\mathrm{CHX}$ group (due to tooth staining)

Selective reporting (re- High risk
porting bias)

\begin{abstract}
No baseline measure of gingival health was provided. No measures of variance were reported. Mean plaque scores were reported only in graphs. The mean supra- and subgingival calculus scores were not reported. We estimated the standard deviation for gingivitis (measured using the $\mathrm{Gl}$ ) as the median of the SDs in the placebo/control groups from similar studies that also used the GI at 6 months so that we could include the gingivitis data in the meta-analyses
\end{abstract}


Banting 1989 (Continued)

Other bias Unclear risk
Quotes: "One examiner was used for each of the soft-tissue, gingivitis and plaque measurements." "...two examiners were used for the pocket depth assessments." No information is provided on intraexaminer reproducibility

Subjects residing in the same household were assigned to the same treatment group. The number of participants who resided in the same household is not reported. The potential effect of clustering was not considered in the analysis and is not clear

Participant characteristics are not described

Bhat 2014

\section{Study characteristics}

\section{Methods}

Trial design: parallel ( 3 arms - 2 arms relevant to this review)

Location: Department of Public Health Dentistry, Darshan Dental College and Hospital, Udaipur, Rajasthan, India

Number of centres: 1

Study duration: recruitment period not reported. Study duration and duration of rinsing was 4 weeks
Participants: undergraduate dental students

Inclusion criteria: quote: "The dental students with age group of 18-24 years, students willing to participate and with dentition of $\geq 20$ teeth and a minimum of 5 teeth per quadrant, no relevant medical history and no periodontal treatment during past 3 months were included"

Exclusion criteria: quote: "Students with orthodontic appliances or severe misaligned

teeth, receiving antibiotic therapy or medication within past 6 months, presence of any systemic illness, subjects availing oral prophylaxis since past 6 months and students unable to comply with study appointment schedule were excluded"

Baseline gingivitis: GI Löe \& Silness 1963, Gp A: mean 0.77 (SD 0.43); Gp B: mean 0.62 (SD 0.52); Gp C: mean 0.91 (SD 0.84)

Age at baseline (years): Gp A: mean 20.95 (SD 0.1); Gp B: mean 20.2 (SD 0.7); Gp C: 20.79 (SD 1.2). Range $18-24$

Gender: male 37 (51\%), female 35 (49\%), not broken down by group

Number randomised: total 72 (Gp A: 24; Gp B: 24; Gp C: 24)

Number evaluated: total 66 (Gp A: 22; Gp B: 22; Gp C: 22)

Interventions

\section{Comparison: $\mathrm{CHX}$ (ClohexPlus) rinse versus herbal (Hiora) rinse* versus control (Nirlife) rinse}

Gp A ( $n=24):$ CHX (0.20\%) $10 \mathrm{~mL}$, twice daily, 60 seconds, 4 weeks

${ }^{*} \mathrm{Gp} \mathrm{B}(\mathrm{n}=24)$ : herbal (Hiora) rinse $10 \mathrm{~mL}$, twice daily, 60 seconds, 4 weeks. We excluded this arm from the risk of bias and analysis

Gp C ( $\mathrm{n}=24)$ : control ( $0.05 \%$ saline) $10 \mathrm{~mL}$, twice daily, 60 seconds, 4 weeks

Prophylaxis at baseline: not reported

OHI: quote: "All the participants were instructed to follow their routine oral hygiene practices along with the assigned regimen and to maintain a reminder sheet on daily product use" 
Bhat 2014 (Continued)

Supervised and non-supervised rinsing: quote: "Each one of the daily rinses was supervised on each weekday and also supervised by a daily recall message for reminding them to use the assigned mouthwash"

Timing of mouthrinsing in relation to toothbrushing: after toothbrushing

Postrinsing instructions: not reported

Outcomes Gingivitis: (gingival inflammation: GI Löe \& Silness 1963); plaque (TQH Turesky 1970); adverse effects (burning sensation, dryness, taste disturbance, discolouration) assessed at 4 weeks

\begin{tabular}{ll}
\hline Funding & Quote: "Research funding: None declared" \\
\hline Notes & $\begin{array}{l}\text { Sample size calculation: quote: "From the pilot study, it was found that the overall prevalence of } \\
\text { plaque and gingivitis was } 80 \% . \text { Considering for the drop outs logistic and technical problems the sam- } \\
\text { ple size was inflated by } 20 \%, \text { hence the sample size was } 72 \text { with } 24 \text { participants in each group" }\end{array}$
\end{tabular}

Adverse effects: a majority of the adverse effects resulted in $\mathrm{Gp} \mathrm{A}$ (CHX group). It was observed that most of the study participants in Gp A complained of mild discolouration 18 (81.8\%), moderate discolouration $2(9.1 \%)$ and dry mouth $18(81.8 \%)$, whereas taste disturbance was higher in Gp C 14 $(63.6 \%)$ as compared to Gp A 10 (45.4\%). The least taste disturbance was seen in Gp B 2 (9.1\%). The burning sensation was highest in Gp A $11(50 \%)$ as compared to Gp B $9(40.9 \%)$ and absent in Gp C

Declarations/conflicts of interest: quote: "Authors' conflict of interest disclosure: The authors stated that there are no conflicts of interest regarding the publication of this article"

\section{Risk of bias}

\section{Bias \\ Authors' judgement Support for judgement}

Random sequence genera- Low risk tion (selection bias)

Quote: "... All the subjects were provided with their assigned mouthrinses and were divided into Group A, Group B and Group C randomly using simple lottery method with 24 participants in each group"

Comment: if "simple lottery method" corresponds to drawing lots then we can consider risk of bias to be low

\begin{tabular}{|c|c|c|}
\hline $\begin{array}{l}\text { Allocation concealment } \\
\text { (selection bias) }\end{array}$ & Unclear risk & $\begin{array}{l}\text { Quote: "The assignment of the participants to the groups and the codes to the } \\
\text { product was done by a person not involved in the examination" }\end{array}$ \\
\hline
\end{tabular}

Comment: insufficient information is provided

$\begin{array}{ll}\begin{array}{l}\text { Blinding of participants } \\ \text { and personnel (perfor- }\end{array} & \text { Quigh risk } \\ \begin{array}{ll}\text { mance bias) } & \text { by ensuring a total subject masking. The examiner and the participants were } \\ \text { All outcomes } & \text { also blinded with regard to the mouthrinse allocated to them thereby ensur- } \\ & \text { ing a double-blinded study" and "Normal saline was coloured to resemble } \\ & \text { the mouthwashes." A burning sensation was reported by almost half of the } \\ & \text { participants in the } 2 \text { active mouthrinse groups. } 81.8 \% \text { of study participants in } \\ & \text { the CHX group complained of mild discolouration and } 9.1 \% \text { of moderate dis- } \\ \text { colouration }\end{array}\end{array}$

Comment: higher levels of tooth staining were reported by most of the study participants in the $\mathrm{CHX}$ group. The higher level of staining in the $\mathrm{CHX}$ group meant that participants could have worked out which group they were in and this could have affected their oral health behaviours

$\begin{array}{lll}\begin{array}{l}\text { Blinding of outcome as- } \\ \text { sessment (detection bias) }\end{array} & \text { High risk } & \begin{array}{l}\text { Quote: "The examiner and the participants were also blinded with regard to } \\ \text { the mouthrinse allocated to them thereby ensuring a double-blinded study." } \\ \text { All outcomes }\end{array} \\ \begin{array}{l}\text { A burning sensation was reported by almost half of the participants in the } 2 \\ \text { active mouthrinse groups. } 81.8 \% \text { of study participants in the CHX group com- } \\ \text { plained of mild discolouration and } 9.1 \% \text { of moderate discolouration }\end{array}\end{array}$


Bhat 2014 (Continued)

Comment: the higher level of staining in the $\mathrm{CHX}$ group meant that the outcome assessor could not be adequately blinded and this could have affected the outcome assessment. The direction of this potential bias is unclear

Incomplete outcome data Low risk (attrition bias)

All outcomes
Attrition after 4 weeks: $4 / 48$ (8.3\%). By group: $\mathrm{CHX} 2 / 24$ (8.3\%), control 2/24 (8.3\%). Reasons for losses not broken down by group: quote: "Two students did not report after 1 month in spite of several recalls, three students were irregular in using mouthwash found during surprise visit and one of the students became ill and had to quit the study due to antibiotic coverage"

Comments: losses are reasonably low and are balanced across groups

\begin{tabular}{|c|c|c|}
\hline $\begin{array}{l}\text { Selective reporting (re- } \\
\text { porting bias) }\end{array}$ & High risk & $\begin{array}{l}\text { Standard deviations of mean scores for gingivitis (CHX group only) are not re- } \\
\text { ported at } 4 \text { weeks. We used the SD for the } \mathrm{CHX} \text { group at baseline so that we } \\
\text { could include the gingivitis data (measured using the GI) in the meta-analyses. } \\
\text { Adverse effects including 'discolouration' are reported (proportions with each } \\
\text { adverse effect by group) }\end{array}$ \\
\hline
\end{tabular}

Other bias Low risk

Quote: "The kappa statistical analysis for inter examiner variability for Turesky et al. Plaque index and Loe and Silness gingival index was 0.89 and 0.97 , whereas the intra examiner was 0.90 and 0.94 respectively showing high degree of conformity in observational judgment. All recordings were made by a single examiner"

No statistically significant difference in mean age, mean baseline plaque scores and mean baseline gingival index scores

Brecx 1993

\section{Study characteristics}

Methods

Trial design: parallel ( 3 arms - 2 arms relevant to this review)

Location: assumed to be at the Health Science Campus, University of Manitoba, Canada

Number of centres: 1

Study duration: recruitment period is not stated. Study duration and duration of rinsing was 3 months

Participants

Participants: quote: "Either medical or physiotherapy students, as well as non-dental laboratory staff, from the Health Science Campus, University of Manitoba"

Inclusion criteria: healthy adults

Exclusion criteria: not reported

Baseline gingivitis: (GI) Gp A: mean 0.6 (no SD); Gp B: mean 0.6 (no SD); Gp C: mean 0.6 (no SD)

Age at baseline (years): range 21-33

Gender: male 15 (42\%), female 21 (58\%), breakdown by group group data not reported, but gender distribution stated to be 'very similar' across treatment groups

Number randomised: 36 (assumed Gp A: 12; assumed Gp B: 12; assumed Gp C: 12)

Number evaluated: 31 (Gp A: 10; Gp B: 9; Gp C: 12)

Interventions

\section{Comparison: $\mathrm{CHX}$ gluconate solution versus Meridol rinse* versus placebo rinse}

Gp A ( $n=12):$ CHX 0.2\%,10 mL, twice daily, 1 min, 3 months 
Brecx 1993 (Continued)

${ }^{\star} \mathrm{Gp} \mathrm{B}(\mathrm{n}=12)$ : Meridol, $10 \mathrm{~mL}$, twice daily, 1 min, 3 months. We excluded this arm from the risk of bias and analysis

$\mathrm{Gp} \mathrm{C} \mathrm{(} \mathrm{n}=12)$ : placebo (quinine-hydrochloride solution), $10 \mathrm{~mL}$, twice daily, $1 \mathrm{~min}, 3$ months

Prophylaxis at baseline: quote: "In the pretreatment phase, the individuals were subjected to a thorough prophylaxis, but not instructed in any form of mechanical plaque control." Subjects had teeth professionally cleaned and continued their usual oral hygiene for a period of 2 weeks until "month 0 " at which time their teeth were polished again. They received a new toothbrush (Butler 411) for the 2 weeks preceding the beginning of month 0

OHI: none given. Quote: "..but not instructed in any form of mechanical plaque control"

Non-supervised rinsing

Timing of mouthrisning in relation to toothbrushing: quote: "The participants were instructed to make sure to rinse twice daily regardless of their brushing habits. However, if they did brush, they were asked to use the mouthrinse after their toothbrushing"

Outcomes

Gingivitis (gingival inflammation: GI Löe \& Silness 1967); plaque (PI Silness \& Löe 1964), assessed at day 14 , month 0 , and after 1,2 , and 3 months of rinsing in addition to usual tooth cleaning

Tooth stain (Modification of the Discolouration Index, Lang \& Räber 1981). Time period not explicitly stated

Dental plaque for vital fluorescence (VF) examination was collected at months $0,1,2$, and 3 (not relevant to this review)

\begin{tabular}{ll}
\hline Funding & Quote: "This investigation was supported by a grant from GABA International Ltd, Therwil, Switzerland" \\
\hline Notes & Sample size calculation: not reported \\
& $\begin{array}{l}\text { Adverse effects: tooth staining i.e. higher DI scores in Meridol and CHX groups than placebo at end of } \\
\text { study. At all time points the Meridol group showed less staining than the CHX group } \\
\text { Declarations/conflicts of interest: none stated }\end{array}$
\end{tabular}

\section{Risk of bias}

\begin{tabular}{|c|c|c|}
\hline Bias & Authors' judgement & Support for judgement \\
\hline $\begin{array}{l}\text { Random sequence genera- } \\
\text { tion (selection bias) }\end{array}$ & Unclear risk & $\begin{array}{l}\text { Quote: "The subjects were randomly assigned to three groups.." } \\
\text { Comment: no description of the method of random sequence generation }\end{array}$ \\
\hline $\begin{array}{l}\text { Allocation concealment } \\
\text { (selection bias) }\end{array}$ & Unclear risk & No information provided \\
\hline $\begin{array}{l}\text { Blinding of participants } \\
\text { and personnel (perfor- } \\
\text { mance bias) } \\
\text { All outcomes }\end{array}$ & High risk & $\begin{array}{l}\text { Quotes: "double-blind", "..all solutions had the same color and were kept in } \\
\text { the same kind of bottle (PET white, opaque)." A placebo containing } 0.02 \% \text { qui- } \\
\text { nine-hydrochloride solution was used } \\
\text { Quote: "The DI increased with time in all groups... At the end of the study, the } \\
\text { Meridol and chlorhexidine groups exhibited higher scores ( } \mathrm{p}<0.001 \text { ) than the } \\
\text { placebo group" } \\
\text { Comment: the higher level of tooth staining in the CHX group meant that par- } \\
\text { ticipants could have worked out which group they were in and this could have } \\
\text { affected their oral health behaviours and hence the outcome. The direction of } \\
\text { this potential bias is unclear }\end{array}$ \\
\hline
\end{tabular}


Brecx 1993 (Continued)

Blinding of outcome as- High risk sessment (detection bias)

All outcomes
Quotes: "double-blind", "All readings were noted by the same investigator.." and "The DI increased with time in all groups... At the end of the study, the Meridol and chlorhexidine groups exhibited higher scores $(p<0.001)$ than the placebo group"

Comment: the higher level of staining in the $\mathrm{CHX}$ group meant that the outcome assessor could not be adequately blinded and this could have affected the outcome assessment. The direction of this potential bias is unclear

\section{Incomplete outcome data High risk} (attrition bias)

All outcomes
Assuming equal numbers in groups at the start (as this was not reported) attrition after 3 months was 2/24 (13.9\%). Attrition by group was 2/12 (16.7\%) CHX; no losses from placebo group. Reasons not broken down by group. Reasons for losses (all study arms): quote: "One subject decided to stop participation, and four others were dismissed for multiple evidence of non-compliance (noshow without several calls, no rinse during $1 \mathrm{~min}$, too much solution left in the bottle, etc)"

Comment: there were differential group losses with losses from the $\mathrm{CHX}$ group and none from the placebo group. The number of participants lost over the 3 months is small but because the groups were small at baseline the proportionate losses are high
Mean scores for tooth staining at 4 weeks were not reported in the text and were read off graphs. Information on variance was only reported graphically as standard error bars on the graphs. Therefore, we estimated the standard error for gingivitis, plaque and tooth staining, from the graphs and used this information to calculate the standard deviations of the mean scores so that we could include the data in the meta-analyses. Apart from tooth staining, adverse effects were not mentioned

Other bias Unclear risk

Quote: "All readings were noted by the same investigator, with an intra-individual variation below $5 \% "$

Comment: the examiner reproducibility is good

Quote: "The periodontal status at time of selection (day-14) as well as age range and gender distribution were very similar..."

Comment: no supporting data are provided

The mean GI scores for the 3 groups at baseline appear similar. The mean DI scores for the 3 groups at baseline also appear similar (on the graph). The mean plaque scores in the placebo group at baseline (on the graph) appear higher than in the $\mathrm{CHX}$ group. It is difficult to assess the baseline balance as the data are not reported or commented on in the text

Brightman 1991

\section{Study characteristics}

Methods

Trial design: parallel (2 arms)

Location: assumed to be at Case Western Reserve University School of Dentistry, Cleveland, Ohio, USA

Number of centres: 1

Study duration: recruitment period not stated. Study duration and duration of rinsing was 3 months 
Brightman 1991 (Continued)

Participants
Participants: quote: "Subjects for this study were selected from the Department of Orthodontics at Case Western Reserve University School of Dentistry, Cleveland, Ohio"

Inclusion criteria: quote: "Forty-four subjects qualified on the basis of the following criteria: (1) They were under-going full-banded edgewise extraction treatment with brackets on their anterior teeth and bands on their molars; (2) four premolars had been extracted; (3) they were at least 11 years of age and no more than 17 years of age; (4) evidence of gingivitis was present, but there was no evidence of periodontitis; (5) there was no evidence of decalcification on their teeth; (6) there was no known hypersensitivity to chlorhexidine; (7) there were no known medical problems or evidence of current antibiotic therapy; and (8) no anterior composites were present"

Exclusion criteria: not specifically stated but are implicit in the inclusion criteria

Baseline gingivitis: not reported

Age at baseline (years): Gp A: mean 14.88 (SD 1.78); Gp B: mean 14.78 (SD1.52); range 11-17

Gender: overall: male (42\%), female (58\%). Group data not specified

Number randomised: 36 (Gp A: 18, Gp B: 18)

Number evaluated: 34 (Gp A: 16, Gp B: 18)

\section{Comparison: $\mathrm{CHX}$ mouthrinse versus placebo mouthrinse}

Gp A ( $=18)$ : CHX (Peridex) $0.12 \%, 1 / 2$ ounce, twice daily, 30 seconds, 3 months

Gp B ( $n=18)$ : placebo (identical to test mouthrinse but without the $\mathrm{CHX}$ ), 1/2 ounce, twice daily, 30 seconds, 3 months

Prophylaxis at baseline: quote: "Each of the subjects was given a thorough prophylaxis and instructed in oral hygiene procedures"

OHI: quotes: "Participants were provided with 3 toothbrushes (one for each month of the study) and 3 tubes of toothpaste." "All subjects were instructed to use the Bass technique of toothbrushing and to brush with the Crest toothpaste that was provided." "The patients were all instructed to use one toothbrush every month. They were to brush only with Crest toothpaste with fluoride, once in the morning after breakfast and once in the evening before bedtime. They were all instructed to brush a minimum of 3 minutes to ensure thorough brushing"

Non-supervised rinsing

Timing of mouthrinsing in relation to toothbrushing: after brushing

Postrinsing instructions: no liquid or food to be taken after using mouthrinse for at least 30 minutes

Outcomes

Gingivitis (gingival inflammation: GI Silness \& Löe 1964 and 1967; gingival bleeding: Eastman Interdental Bleeding Index, Caton and Polson 1985); plaque (PI Löe \& Silness 1964 and 1967); staining intensity and area (method created at Case Western Reserve University School of Dentistry, Department of Periodontics); assessed at 6 weeks, and 3 months follow-up

Funding Funding: not reported

Notes

Sample size calculation: quote: "This study was designed to provide a minimal power of $87 \%$ for detecting a clinically important difference to be statistically significant at the 0.05 probability level"

Adverse effects: quote: "Both the chlorhexidine group and the placebo group showed significant increases in the degree of stain, but the chlorhexidine group had a more significant amount of stain"

Declarations/conflict of interest: none stated

\section{Risk of bias}


Brightman 1991 (Continued)

\section{Bias Authors' judgement Support for judgement}

Random sequence genera- Low risk tion (selection bias)
Quote: "Two tables of random numbers, one for the male population and one for the female population, were used, and the subjects were thus assigned to one of two treatment groups"

Comment: adequate method of random sequence generation

\begin{tabular}{lll}
\hline $\begin{array}{l}\text { Allocation concealment } \\
\text { (selection bias) }\end{array}$ & Unclear risk & No information is provided
\end{tabular}

(selection bias)

\section{Blinding of participants High risk} and personnel (perfor-

mance bias)

All outcomes
Quote: "These measures (use of random number tables) ensured that neither the investigators nor the patients knew whether participants were in the experimental group or the placebo group"

Comment: the authors indicated that the participants were blind to group allocation (although the use of a random number table would not have ensured blinding)

Quotes: "The placebo mouthrinse was identical to the experimental mouthrinse except that the placebo mouthrinse did not contain chlorhexidine. The appearance and the taste of both mouthrinses were similar. They were both colored blue, mint flavored, and contained in amber-colored bottles" and "The mean stain severity index for the chlorhexidine group showed significantly more stain at 12 weeks $(p<0.001)$ for all of the sites measured"

Comment: despite the efforts undertaken by the investigators the increased staining in the $\mathrm{CHX}$ group is likely to have compromised the attempted participant blinding

\section{Blinding of outcome as- High risk} sessment (detection bias) All outcomes
Quote: "These measures (use of random number tables) ensured that neither the investigators nor the patients knew whether participants were in the experimental group or the placebo group"

Comment: the authors indicated that the investigators were blind to group allocation (although the use of a random number table would not have ensured blinding)

Quote: "The GI and the PI were scored by the primary investigator, and the stain index and the bleeding index were scored by a second investigator, who was a senior dental student. This was done to minimise bias, so that the investigator who was scoring the $\mathrm{GI}$ and $\mathrm{PI}$ was not influenced by the degree of extrinsic stain, which is a common side effect of chlorhexidine"

Comment: despite the efforts undertaken by the investigators to blind outcome assessment, the primary investigator, although not scoring the stain, would likely still be aware of increased staining in some individuals and this meant that the outcome assessors could not be adequately blinded and this could have affected the outcome assessment. The direction of this potential bias is unclear

Attrition after 3 months was 2/36 (5.6\%). Attrition by group: 2/18 (11.1\%) CHX; no losses from placebo group. Reasons for losses from $\mathrm{CHX}$ group: "inability to cooperate" 2 lost from $\mathrm{CHX}$ mouthrinse group after less than 1 week

Comment: it is not known whether the "inability to cooperate" of the 2 participants lost from the $\mathrm{CHX}$ group were related to use of the $\mathrm{CHX}$ mouthrinse. However, the number of participants lost over the 3 months is small and unlikely to have affected the results 
Brightman 1991 (Continued)

Selective reporting (re- High risk Mean scores for all outcomes are reported graphically with $95 \% \mathrm{Cl}$ bars in the porting bias) graphs. We estimated the mean scores and $95 \% \mathrm{Cls}$ for all outcomes. We used the information on the $95 \%$ Cls to calculate the SDs of the mean scores so that we could include the data in meta-analyses. Apart from tooth staining, adverse effects were not mentioned

Other bias

Unclear risk

The outcomes were measured by 2 investigators one of which was a senior dental student. No information is provided on intraexaminer reproducibility (Interexaminer not relevant as the 2 examiners recorded different variables)

Comment: not enough information is provided

Mean scores for gingivitis, plaque and tooth stain as represented on graphs appear quite balanced. However, the statistical significance of the differences is not reported

\section{Charles 2004}

\section{Study characteristics}

Methods Trial design: parallel ( 3 arms -2 arms are relevant to this review)

Location: assumed research facility, USA

Number of centres: assumed to be 1

Study duration: recruitment period is not stated. Duration of study and duration of rinsing was 6 months

Participants Participants: adults aged 20-57

Inclusion criteria: quote: "a minimum of 20 sound, natural teeth; a mean plaque index (PI) (Turesky et al 1970) of at least 1.95; a mean GI (Loe \& Silness 1963) of at least 0.95"

Exclusion criteria: quote: "Teeth that were grossly carious, fully crowned or extensively restored, orthodontically banded, abutments, or third molars were not included in the tooth count. Subjects with gross oral pathology or who were taking antibiotic or antiinflammatory drugs were excluded"

Baseline gingivitis: (GI), Gp A: mean 1.35 (SE 0.04); Gp B: mean 1.31 (SE 0.04); Gp C: mean 1.27 (SE 0.03)

Age at baseline (years): Gp A: mean 31.4 (SD 9.4); Gp B: mean 32.0 (SD 6.6); Gp C: mean 32.2 (SD 6.3). Range 20-57 (all arms of study)

Gender (reported for completers): overall; male 39 (36\%); female 68 (64\%). Gp A: male 13 (36\%), female 23 (64\%); Gp B: male 12 (35\%), female 22 (65\%); Gp C: male 14 (38\%), female $23(62 \%)$

Number randomised: 108 (Gp A: 36, Gp B: 34, Gp C: 38)

Number evaluated: 107 (Gp A: 36, Gp B: 34, Gp C: 37)

Interventions

\section{Comparison: $\mathrm{CHX} 0.12 \%$ (Peridex) versus EO (essential oil mouthrinse, Listerine antiseptic) ${ }^{\star}$ ver- sus control ( $5 \%$ hydroalcohol negative control)}

Gp A ( $\mathrm{n}=36)$ : CHX (Peridex) 0.12\%,15 mL, twice daily, 30 seconds, 6 months

${ }^{*} \mathrm{Gp} \mathrm{B}(\mathrm{n}=34)$ : Listerine, $20 \mathrm{~mL}$, twice daily, 30 seconds, 6 months. We excluded this arm from the risk of bias and analysis

Gp C ( $n=38)$ : control ( $5 \%$ hydroalcohol), $20 \mathrm{~mL}$, twice daily, 30 seconds, 6 months 
Prophylaxis at baseline: quote: "Following the baseline examination, each subject received a complete dental prophylaxis to remove all plaque, calculus, and extrinsic stain"

OHI: none given. Quote: "....subjects followed their usual oral hygiene and dietary habits.." Soft nylon toothbrushes and fluoridated toothpaste were provided to all subjects and replenished periodically as need for the duration of the study

Quote: "One of the daily rinses was supervised on each weekday"

Postrinsing instructions: not reported

Timing of mouthrinsing in relation to toothbrushing: quote: "Rinsings were not done at time of toothbrushing but at separate times"

Outcomes

Gingivitis (gingival inflammation: GI Löe \& Silness 1963; gingival bleeding: \% bleeding sites (GI scores 2 or 3 indicating bleeding)); plaque (TQH, Turesky 1970); extrinsic tooth stain (Lobene Discolouration Index, Lobene 1968); supragingival calculus (Volpe-Manhold Calculus Index, Manhold 1965, Volpe 1965, 1967, Barnett 1989), assessed at 3 and 6 months follow-up

Funding

Funding: the authors are affiliated to Dental Products Testing and Pfizer

Notes

Sample size calculation: quote: "This study was designed to provide a minimal power of 0.80 for detecting a statistically significant difference in plaque and gingivitis scores at the 0.05 probability level"

Adverse effects: during the course of the study no oral mucosal lesions that could be attributed to any of the test mouthrinses were observed"

Smokers: by group Gp A: 8/36 (22\%); Gp B: 8/34 (24\%); Gp C: 6/38 (16\%) (differences are not statistically significant)

Declarations/conflicts of interest: the authors are affiliated with Dental Products Testing and Pfizer. No explicit conflicts of interest were declared

\section{Risk of bias}

Bias Authors' judgement Support for judgement

Random sequence genera- Low risk tion (selection bias)
Quotes: "Subjects were randomised into three groups", "...assigned to either one of two test groups or a negative control group according to a computer generated random code"

Comment: adequate method of random sequence generation

Allocation concealment $\quad$ Unclear risk No information is provided
(selection bias)

\section{Blinding of participants High risk} and personnel (performance bias)

All outcomes

Quote: "One of the daily rinses was supervised on each weekday." No mention of blinding of study participants

Comment: the study compared $\mathrm{CHX}$ with essential oil and control ( $5 \%$ hydroalcohol) and different volumes of the rinses were used making participant and personnel blinding difficult to achieve. Lack of blinding could have affected participants' oral health behaviours and hence the outcome but direction of this potential bias is unclear

Blinding of outcome assessment (detection bias) All outcomes
High risk

Quotes: "observer blind" and "At 3 and 6 months, there was significantly more gingival region stain in the essential oil group $(p<0.05)$ and the chlorhexidine group $(p<0.001)$ compared with the control group.." 
Comment: the higher level of staining in the $\mathrm{CHX}$ group meant that the outcome assessor could not be adequately blinded and this could have affected the outcome assessment. The direction of this potential bias is unclear

Incomplete outcome data Low risk (attrition bias)

Only 1 participant was lost over the 6 months. Attrition by group $1 / 38(2.63 \%)$ placebo group. No losses from $\mathrm{CHX}$ group. Reasons for losses: personal reasons. Lost after the 3-month exam and results for this individual were included in the 3-month exam

Comment: the reason the individual was lost was not related to the intervention

Selective reporting (re- High risk
porting bias)

We calculated standard deviations for gingivitis and plaque from the standard errors reported in the tables. We could not include data on \% bleeding sites in meta-analyses as the data were reported for total number of sites rather than individuals. Information on the variance of the mean calculus and stain scores was not reported and those outcomes could not be included in meta-analyses

Other bias Low risk

Quotes: "All examinations were conducted by a single, experienced dental examiner. Reliability was established for the gingival index (GI) with a $\mathrm{k}$ statistic of 0.75 , which indicates satisfactory calibration."

"There were no statistically significant differences among the treatment groups with respect to age, gender, smoking.." There were no statistically significant differences among the treatment groups in relation to the gingival and calculus indices. The plaque index was significantly lower in the control group than in the $\mathrm{CHX}$ group at baseline

Comment: all participants had a baseline prophylaxis to remove all plaque, calculus and extrinsic stain. It can be assumed that they were similar with respect to plaque and stain at the start of the study

Chaves 1994

\section{Study characteristics}

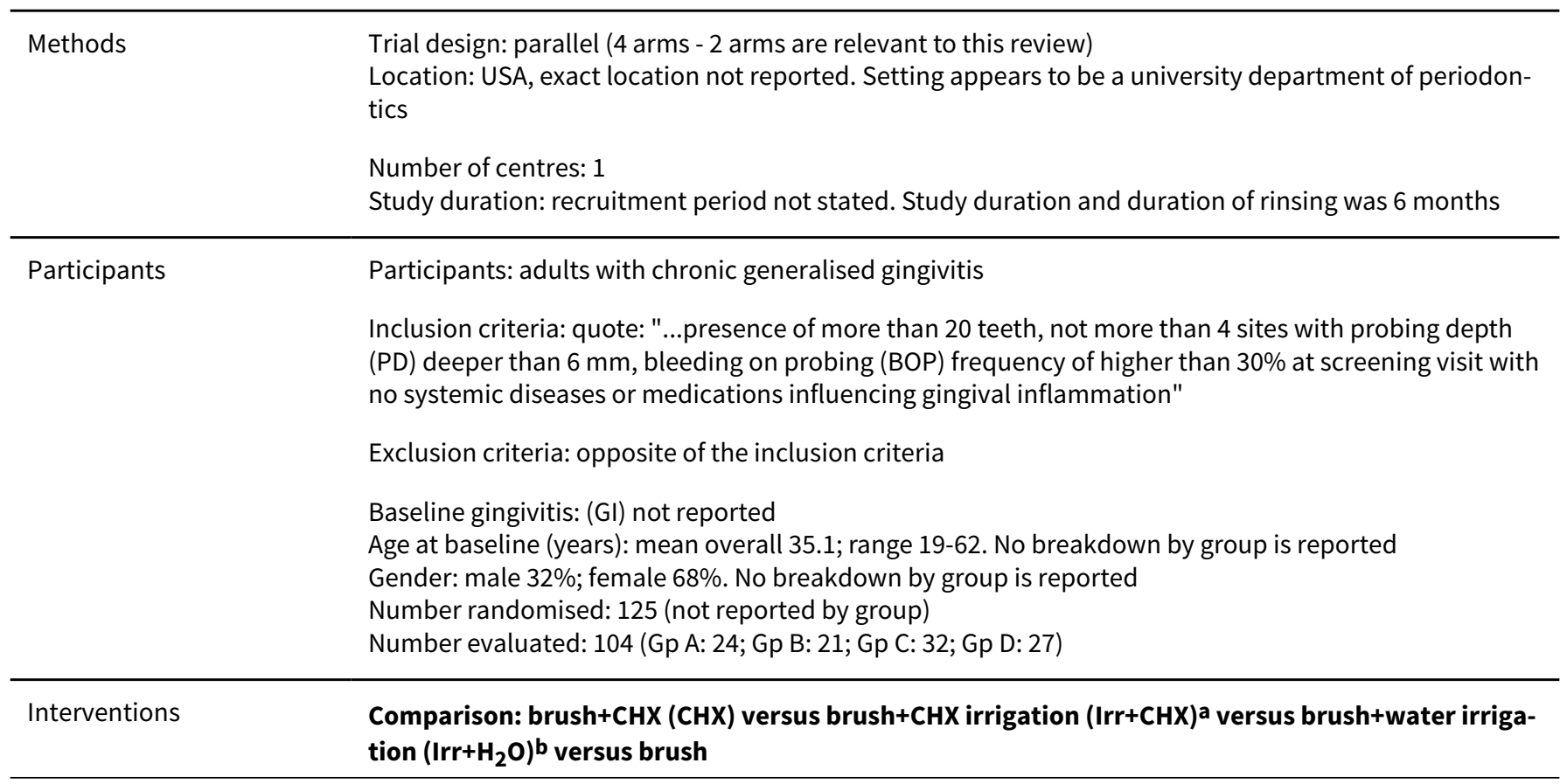


aGp B ( $n=21): C H X 0.04 \%$ Irr, $400 \mathrm{~mL}$, once daily, no duration reported, 6 months. We excluded this arm from our risk of bias and analysis

bGp C ( $n=32): 1 r r+H_{2} \mathrm{O}, 400 \mathrm{~mL}$, once daily, no duration reported, 6 months. We excluded this arm from our risk of bias and analysis

Gp D ( $n=27):$ brush, N/A

Note: Numbers stated above relate to completers. No figures were reported by group for those who were randomised

Prophylaxis at baseline: yes, but no details reported

OHI: quote: "After baseline data collection, all patients received instructions on the use of a toothbrush and dental floss by watching a standardized video tape." "[after prophylaxis] further oral hygiene instructions specific to the treatment group [were provided]"

Postrinsing instructions: not reported

Non-supervised rinsing

Timing of mouthrinsing in relation to toothbrushing: not reported

Outcomes

Gingivitis (gingival inflammation: GI Löe 1963); gingival bleeding: BOP (automated probe (Floride probe) using 20 grams of force, Gibbs et al 1988); plaque (PI Silness \& Löe 1964); assessed at 3 and 6 months follow-up. Probing depth and subgingival microbial samples were taken from sites presenting probing depths $>4 \mathrm{~mm}$ and BOP at baseline visit (not relevant to this review)

Funding $\quad$ Funding: quote: "This study was supported by Teledyne Water Pik, Denver, CO" (USA)

Notes

Sample size calculation: not reported

Adverse effects: quote: "Several of the patients who did not return to the scheduled examination visits reported an unacceptable taste associated with the chlorhexidine. No patient reported adverse reaction or side effects to the mouthrinse ...."

Declarations/conflicts of interest: not reported

Additional information was obtained from Chaves 1993

\section{Risk of bias}

\begin{tabular}{|c|c|c|}
\hline Bias & Authors' judgement & Support for judgement \\
\hline \multirow[t]{2}{*}{$\begin{array}{l}\text { Random sequence genera- } \\
\text { tion (selection bias) }\end{array}$} & Low risk & $\begin{array}{l}\text { Quote: "Patients were randomly assigned to one of four groups based on a } \\
\text { random number table that was computer generated" }\end{array}$ \\
\hline & & Comment: adequate method of random sequence generation \\
\hline $\begin{array}{l}\text { Allocation concealment } \\
\text { (selection bias) }\end{array}$ & Unclear risk & No information is provided \\
\hline \multirow{2}{*}{$\begin{array}{l}\text { Blinding of participants } \\
\text { and personnel (perfor- } \\
\text { mance bias) } \\
\text { All outcomes }\end{array}$} & High risk & $\begin{array}{l}\text { Quote: "The clinical examiners were blinded to the treatment groups, howev- } \\
\text { er dental assistants and hygienists were responsible for providing information } \\
\text { and support to their respective plaque control group" }\end{array}$ \\
\hline & & $\begin{array}{l}\text { Comment: blinding of participants was not possible due to the differences in } \\
\text { the intervention groups being compared. The dental assistants and hygienists } \\
\text { were aware of the group allocations of participants }\end{array}$ \\
\hline
\end{tabular}


Chaves 1994 (Continued)

Blinding of outcome assessment (detection bias) All outcomes
Unclear risk

Quote: "The clinical examiners were blinded to the treatment groups....The clinical examiners did not have access to any of the clinical or compliance records during the study"

Comment: tooth staining is likely to have been noticeable in the $\mathrm{CHX}$ group after 6 months, and in this situation the outcome assessor could have worked out which individuals used $\mathrm{CHX}$ and not be adequately blinded. However, tooth staining is not reported and there is not enough information upon which to base a judgement
Incomplete outcome data High risk (attrition bias)

All outcomes
Attrition after 6 months (all study arms) was 21/125 (16.8\%). Attrition by group is not reported. Reasons for loss to follow-up: several of the patients who did not return to the scheduled exam visits reported an unacceptable taste associated with $\mathrm{CHX}$

Comment: several participants were lost from the $2 \mathrm{CHX}$ groups because they did not like the taste of the rinse. Exact numbers lost from each group are not reported but the number of participants in the $2 \mathrm{CHX}$ groups completing the trial seemed to be appreciably lower than in the 2 groups not using CHX. Assuming the missing participants in 1 group had a higher mean (e.g. gingivitis score) than those in the other groups, as the attrition rate increased, so would the mean difference between groups
Mean scores for all outcomes are reported graphically with SD bars in the graphs. We estimated the mean scores and SDs for all outcomes so that we could include the data in meta-analyses. Information on adverse effects is reported but no specific information relating to tooth staining is reported

Other bias Unclear risk

\begin{abstract}
Quote Chaves 1993: "The clinical examinations were carried out by 6 different examiners with each patient being evaluated by the same examiner at all time periods...prior to the initiation of the study, all examiners participated in group seminars and clinical sessions on the assessment of clinical parameters and the use of the Florida Probe and were compared to a single standard examiner. Although no attempt was made to evaluate inter or intra-examiner reproducibility, no statistically significant differences were found between the examiners when PD, BOP and GI were evaluated.."
\end{abstract}

The groups appear reasonably balanced (on graph) with respect to GI, BOP and $\mathrm{PI}$ at baseline. The actual data at baseline are not reported

Comment: not enough information is provided

Corbet 1997

\section{Study characteristics}

\begin{tabular}{ll}
\hline Methods & $\begin{array}{l}\text { Trial design: parallel (2 arms) } \\
\text { Location: knitting factory assumed to be in Ho Tung, Guangdong, China } \\
\text { Number of centres: assumed to be } 1\end{array}$ \\
& $\begin{array}{l}\text { Study duration: recruitment period not stated. Duration of study } 6 \text { months, but data only reported for } 3 \\
\text { months }\end{array}$ \\
\hline Participants & $\begin{array}{l}\text { Participants: quotes: "All were employed in a knitting factory in the area of Ho Tung, Province of Guang- } \\
\text { dong, People's Republic of China. However, most of the participants were migrant workers and origi- } \\
\text { nated from other Provinces such as Henan, Hebei and Sichuan" and "..a population with established } \\
\text { gingivitis and abundant supra- and subgingival plaque and calculus" } \\
\text { Inclusion criteria: quote: "The subjects were admitted to the study if they were in good general health } \\
\text { and had not been exposed to antibiotic therapy for at least } 6 \text { months. Furthermore, at least } 20 \text { teeth }\end{array}$
\end{tabular}


had to be present. No probing depth of pockets exceeded $6 \mathrm{~mm}$ and no loss of attachment of more

than $2 \mathrm{~mm}$ was encountered"

Exclusion criteria: quote: "Subjects with known allergies, haematological disorders or diabetes mellitus were not allowed to participate. Also, pregnant women were excluded"

Gingivitis (at baseline for the completers): GI: overall mean 1.43 (SE 0.01); Gp A: mean 1.40 (SE 0.02); Gp

B: mean 1.46 (SE 0.01)

Age at baseline (years): range 17-39. Overall mean 22.5 (4.95). Gp A: mean 23.0 (4.34); Gp B: mean 22.3

(5.26)

Gender: overall: male 13 (21.7\%), female 47 (78.3\%). Gp A: male 5 (25\%), female 15 (75\%); Gp B: male 8

(20\%), female $32(80 \%)$

Number randomised: 60 (Gp A: 20; Gp B: 40)

Number evaluated: At 3-month measurement: 36 (Gp A: 13; Gp B: 23)

Interventions

\section{Comparison: $\mathrm{CHX}$ versus placebo control}

Gp A ( $n=20)$ : CHX (Peridex) 0.12\%, 15 mL, twice daily, 45 seconds, 6 days per week for 6 months

$\mathrm{Gp} \mathrm{B}(\mathrm{n}=40)$ : placebo (identical in composition to the test rinse only without the active ingredient), 15 $\mathrm{mL}$, twice daily, 45 seconds, 6 days per week for 6 months

Prophylaxis at baseline: quote: "A unique feature of the present study was that no pre-experimental prophylaxis was performed prior to the commencement of the supervised rinsing"

$\mathrm{OHI}$ : none given. Quote: "No attempts were made to influence the sparsely performed oral hygiene

practices of the participants"

Postrinsing instructions: not reported

Supervision of rinsing: quote: "The supervision of the daily rinses by factory staff assured an almost $100 \%$ compliance and hence, it may be assumed that optimal conditions for a test of the therapeutical principle of chlorhexidine mouthrinses were provided... All rinsings were supervised by one of the supervisors in the factory. The rinsings were performed at $8.00 \mathrm{am}$ and at $7.00 \mathrm{pm}$, and were timed for 45s." Note: there was a 2-week period during the study when participants were not supervised as they returned home during the Chinese New Year holiday. Quote: "During the 2 weeks of the Chinese New Year holiday (January 1995), mouthrinses were provided for the subjects to take to their homes and instructions on their usage was given by the supervisor"

Timing of mouthrinsing in relation to toothbrushing: not reported

$\begin{array}{ll}\text { Gutcomes } & \text { Gingivitis (gingival inflammation: GI Löe \& Silness 1963, Löe 1967; gingival bleeding: sites with scores } \\ & 2 \text { or } 3 \text { indicating bleeding); plaque (PI Silness \& Löe 1964); stain (Discolouration Index Lang \& Räber } \\ \text { 1981); calculus (Calculus Surface Severity Index system (CSSI), Ennever et al 1961); assessed at } 3 \text { and } 6 \\ \text { months follow-up. *Note: Quote: "this report is limited to the 3-month data" }\end{array}$

Funding Quote: "This study was supported by a grant for collaborative research of the Clinical Research Foundation (CRF) for the Promotion of Oral Health, University of Berne, Switzerland"

$\begin{array}{ll}\text { Notes } & \text { Sample size calculation: not reported } \\ & \text { Adverse effects: not reported except for tooth staining and calculus } \\ & \text { Declarations/conflicts of interest: none stated }\end{array}$

\section{Risk of bias}

Bias Authors' judgement Support for judgement

Random sequence genera- Unclear risk tion (selection bias)

Quotes: "Randomised controlled clinical trial", "...subjects were divided into two groups matched according to the mean gingival index"

Comment: no description of the method of random sequence generation

\begin{tabular}{lll}
\hline $\begin{array}{l}\text { Allocation concealment } \\
\text { (selection bias) }\end{array}$ & Unclear risk information is provided
\end{tabular}


Corbet 1997 (Continued)

Blinding of participants and personnel (performance bias)

All outcomes
High risk

Quote: "..double-masked." The placebo rinse was identical in composition to the test rinse only without the active ingredient ( $\mathrm{CHX})$. Rinsings were supervised and blinding of the factory supervisors is not explicitly stated but it is considered unlikely that they were aware of the content of the rinses

Quotes: "The overall increase in mean discoloration index was significantly higher in the Test than the Control group" and "There were significantly ( $p$ 0.05 ) more surfaces scoring $\mathrm{DI}=1,2$ or 3 in the Test group (39\%) than in the control group (28\%)"

Comment: the participants in the $\mathrm{CHX}$ group may have noticed the increased staining and worked out which group they were in and this may have affected their oral health behaviours

\section{Blinding of outcome as- High risk} sessment (detection bias) All outcomes
Quotes: "..double-masked", "The same examiners calibrated for one particular index system scored the same clinical index in all subjects at baseline and after 3 months", "The overall increase in mean discoloration index was significantly higher in the Test than the Control group" and "There were significantly $(p<0.05)$ more surfaces scoring $\mathrm{DI}=1,2$ or 3 in the Test group $(39 \%)$ than in the control group (28\%)"

Comment: it is not clear if the "double-masked" included the examiners. Even if the masking refers to the examiners, it is likely that the examiners were aware of which participants were using $\mathrm{CHX}$ due to the increased tooth staining associated with $\mathrm{CHX}$ rinse
Incomplete outcome data High risk (attrition bias)

All outcomes
Attrition at 3 months was 24/60 (40\%). Attrition by group: $7 / 20$ (35\%) CHX, $17 / 40$ (42.5\%) placebo. Reasons for losses (not broken down by group): the participants were mostly migrant workers from other provinces within China. 19 subjects failed to return from their homes after the Chinese New Year holiday and 5 were excluded due to pregnancy

Quote: "The mean baseline scores for those subjects completing the study were not significantly different for the test and control group, respectively, although they were slightly different when compared with the baseline data of the original study population"

Comment: losses were very high and although the reasons for losses are not related to the intervention the proportion of those lost is so high that a distortion of the true intervention effect cannot be ruled out Selective reporting (re- High risk
porting bias)
We calculated standard deviations for gingivitis (GI), plaque, calculus and staining from the standard errors reported in the text. No information was reported on the variance for gingival bleeding (\% sites scoring $0,1,2$, or 3 ). No information regarding adverse effects apart from tooth staining and calculus were reported. This is a 6-month study but only 3-month data are reported

\begin{tabular}{ll}
\hline Other bias & No information is provided on intra- and interexaminer reproducibility. \\
& $\begin{array}{l}\text { Baseline characteristics of the participants were balanced for } \mathrm{GI}, \mathrm{PI}, \mathrm{CSSI}, \mathrm{DI}, \\
\text { age and gender }\end{array}$
\end{tabular}

de la Rosa 1988

\section{Study characteristics}

Methods

Trial design: parallel ( 2 arms)

Location: a boarding school in Monterrey, Mexico 
de la Rosa 1988 (Continued)

Number of centres: 1

Study duration: recruitment period not stated. Duration of study and duration of rinsing was 12 weeks

Participants: boarding school boys
Inclusion criteria: quote: "For acceptance into the study, they had to have some degree of plaque-in-
duced gingivitis"
Exclusion criteria: quote: "Those with gross oral neglect or pathoses that needed prompt care were not
accepted"
Baseline gingivitis: (Papillary Marginal Gingivitis Index; mean baseline score of all sites graded for indi-
viduals examined at 6 weeks) Gp A: mean 0.6933; Gp B: mean 0.6513
Age at baseline (years): range 8-18
Gender: $100 \%$ male
Number randomised: not reported
Number evaluated: 92 (Gp A: 46; Gp B: 46)

Interventions

\section{Comparison: $\mathrm{CHX}$ versus placebo}

Gp A (completers $n=46)$ : CHX 0.12\%, $15 \mathrm{~mL}$, twice daily, 30 seconds, 12 weeks

Gp B (completers $n=46)$ : placebo (composition not described), $15 \mathrm{~mL}$, twice daily, 30 seconds, 12 weeks

Prophylaxis at baseline: quote: "...all subjects received a dental prophylaxis to remove any dental accretions"

OHI: quote: "At the beginning of the studies, the subjects received soft toothbrushes and brushing instructions"

Postrinsing instructions: not reported

Supervised rinsing

Timing of mouthrinsing in relation to toothbrushing: quote: "The evening rinsing was preceded by a one-minute toothbrushing with a sodium fluoride dentifrice, Crest"

\section{Outcomes}

Gingivitis (gingival inflammation: Papillary Marginal Gingivitis Index, de la Rosa and Sturzenberger 1976 , severity (mean score all sites) and occurrence (proportion of sites with a PMGI score $\geq 1$ )); plaque (TQH, Turesky 1970); assessed at 6 and 12 weeks follow-up

Funding Funding: quote: "The work was supported by a grant of the Procter \& Gamble Company, Cincinnati, $\mathrm{OH}^{\prime \prime}$

\section{Notes}

Sample size calculation: not reported

Adverse effects: quotes: "Some mild epithelial desquamations were observed in some chlorhexidine subjects... There was no discomfort reported in association with these transient incidences in our study. Neither was there a consequence on oral health... there was an increase in extrinsic tooth stain and supragingival calculus in the chlorhexidine groups.." and "There was no lasting side effect on the oral soft tissue or the health of the users"

Declarations/conflicts of interest: potential conflict of interest: the second author is a Senior Dentist at the Procter and Gamble Company and the third author is an Associate Director, Peridex Research, Procter and Gamble Company

\section{Risk of bias}

Bias Authors' judgement Support for judgement

Random sequence genera- Unclear risk tion (selection bias)

Quote: "..subjects were stratified by age intervals $(<12$ and $>/=12)$ and stratified by seven intervals of gingivitis scores ranging from $<0.25$ to $>1.50$. Within these strata, the subjects were distributed into the chlorhexidine or the placebo groups at random"

Comment: no description of the method of random sequence generation

Allocation concealment $\quad$ Unclear risk $\quad$ No information is provided
(selection bias)


de la Rosa 1988 (Continued)

Blinding of participants and personnel (performance bias)

All outcomes
High risk

The authors report that the study is "double-blind". The comparison group rinsed with placebo rinse, no details are given regarding the formulation or presentation of the placebo

Comment: it is not clear whether the 'supervisory personnel' who dispensed the toothpaste and mouthrinse and supervised the rinsing were blind.The authors report an increase in extrinsic tooth stain and supragingival calculus in the $\mathrm{CHX}$ group. The higher level of tooth staining and calculus in the $\mathrm{CHX}$ group meant that participants could have worked out which group they were in (or that they were in the active mouthrinse group) and this could have affected their oral health behaviours and hence the outcome. The direction of this potential bias is unclear
Blinding of outcome as- High risk sessment (detection bias) All outcomes

The authors report that the study is "double-blind". Quote: "Previous records were not available at subsequent examinations nor did examiners have any knowledge as to which treatment group a given subject belonged"

Comment: the authors report an increase in extrinsic tooth stain and supragingival calculus in the $\mathrm{CHX}$ group. The higher level of staining and calculus in the $\mathrm{CHX}$ group meant that the outcome assessor could not be adequately blinded and this could have affected the outcome assessment. The direction of this potential bias is unclear

\begin{tabular}{lll}
\hline $\begin{array}{l}\text { Incomplete outcome data } \\
\text { (attrition bias) } \\
\text { All outcomes }\end{array}$ & Unclear risk & $\begin{array}{l}\text { Numbers at baseline not reported, so attrition from baseline cannot be calcu- } \\
\text { lated. Between } 6 \text { weeks and } 3 \text { months } 4(8 \%) \text { participants were lost from the } \\
\text { CHX group and } 6(11.5 \%) \text { participants were lost from the placebo group }\end{array}$ \\
\hline $\begin{array}{l}\text { Selective reporting (re- } \\
\text { porting bias) }\end{array}$ & High risk & $\begin{array}{l}\text { The SDs for the main outcome (mean gingivitis occurrence and severity) are } \\
\text { not reported. The ancillary outcome (plaque) is mentioned but not fully re- } \\
\text { ported possibly because the result was not statistically significant }\end{array}$ \\
\hline Other bias & Unclear risk & $\begin{array}{l}\text { No information is provided on intra- and interexaminer reproducibility. } \\
\text { The groups appear balanced for gingivitis at baseline. The authors state that } \\
\text { the baseline scores demonstrate comparability of the test and control groups. } \\
\text { However, the statistical significance of the differences is not reported }\end{array}$
\end{tabular}

de la Rosa 1988b

\section{Study characteristics}

\begin{tabular}{|c|c|}
\hline Methods & $\begin{array}{l}\text { Trial design: parallel ( } 2 \text { arms) } \\
\text { Location: a boarding school in Monterrey, Mexico } \\
\text { Number of centres: } 1 \\
\text { Study duration: recruitment period is not stated. Study duration and duration of rinsing was } 10 \text { weeks }\end{array}$ \\
\hline Participants & $\begin{array}{l}\text { Participants: boarding school boys } \\
\text { Inclusion criteria: quote: "For acceptance into the study, they had to have some degree of plaque-in- } \\
\text { duced gingivitis" } \\
\text { Exclusion criteria: quote: "Those with gross oral neglect or pathoses that needed prompt care were not } \\
\text { accepted" } \\
\text { Baseline gingivitis: (Papillary Marginal Gingivitis Index; mean score of all sites graded at baseline for } \\
\text { those examined at } 10 \text { weeks) Gp A: mean } 0.4544 ; \text { Gp B: mean } 0.4263 \\
\text { Age at baseline (years): range } 8-18 \\
\text { Gender: } 100 \% \text { male } \\
\text { Number randomised: not reported } \\
\text { Number evaluated: } 99 \text { (Gp A: } 49 ; \text { Gp B: } 50)\end{array}$ \\
\hline
\end{tabular}


de la Rosa 1988b (Continued)

Interventions

\section{Comparison: $\mathrm{CHX}$ versus placebo}

Gp A (completers $n=49$ ): $\mathrm{CHX} 0.12 \%$ (Peridex), $15 \mathrm{~mL}$, twice daily, 30 seconds, 10 weeks

Gp B (completers $n=50$ ): placebo (composition not described), $15 \mathrm{~mL}$, twice daily, 30 seconds, 10 weeks

Prophylaxis at baseline: quote: "...all subjects received a dental prophylaxis to remove any dental accretions"

OHI: quote: "At the beginning of the studies, the subjects received soft toothbrushes and brushing instructions"

Postrinsing instructions: not reported

Supervised rinsing

Timing of mouthrinsing in relation to toothbrushing: quote: "The evening rinsing was preceded by a one-minute toothbrushing with a sodium fluoride dentifrice, Crest"

\begin{tabular}{|c|c|}
\hline Outcomes & $\begin{array}{l}\text { Gingivitis (gingival inflammation: Papillary Marginal Gingivitis Index, de la Rosa and Sturzenberger } \\
\text { 1976; severity (mean score all sites) and occurrence (proportion of sites with a PMGI score } \geq 1 \text { ); assessed } \\
\text { at } 10 \text { weeks follow-up } \\
\text { Plaque (TQH, Turesky 1970) }\end{array}$ \\
\hline Funding & $\begin{array}{l}\text { Funding: quote: "The work was supported by a grant of the Procter \& Gamble Company, Cincinnati, } \\
\text { OH" }\end{array}$ \\
\hline Notes & $\begin{array}{l}\text { Sample size calculation: not reported } \\
\text { Adverse effects: quotes: "Some mild epithelial desquamations were observed in some chlorhexidine } \\
\text { subjects... There was no discomfort reported in association with these transient incidences in our } \\
\text { study. Neither was there a consequence on oral health... there was an increase in extrinsic tooth stain } \\
\text { and supragingival calculus in the chlorhexidine groups.." and "There was no lasting side effect on the } \\
\text { oral soft tissue or the health of the users" } \\
\text { Declarations/conflicts of interest: potential conflict of interest: the second author is a Senior Dentist at } \\
\text { the Procter and Gamble Company and the third author is an Associate Director, Peridex Research, Proc- } \\
\text { ter and Gamble Company }\end{array}$ \\
\hline
\end{tabular}

\section{Risk of bias}

Bias Authors' judgement Support for judgement

Random sequence genera- Unclear risk tion (selection bias)

Quote: "..subjects were stratified by age intervals $(<12$ and $>/=12)$ and stratified by seven intervals of gingivitis scores ranging from $<0.25$ to $>1.50$. Within these strata, the subjects were distributed into the chlorhexidine or the placebo groups at random"

Comment: no description of the method of random sequence generation

\begin{tabular}{|c|c|c|}
\hline $\begin{array}{l}\text { Allocation concealment } \\
\text { (selection bias) }\end{array}$ & Unclear risk & No information is provided \\
\hline \multirow{2}{*}{$\begin{array}{l}\text { Blinding of participants } \\
\text { and personnel (perfor- } \\
\text { mance bias) } \\
\text { All outcomes }\end{array}$} & High risk & $\begin{array}{l}\text { The authors report that the study is "double-blind". The comparison group } \\
\text { rinsed with placebo rinse, no details are given regarding the formulation or } \\
\text { presentation of the placebo }\end{array}$ \\
\hline & & $\begin{array}{l}\text { Comment: it is not clear whether the 'supervisory personnel' who dispensed } \\
\text { the toothpaste and mouthrinse and supervised the rinsing were blind. The au- } \\
\text { thors report an increase in extrinsic tooth stain and supragingival calculus in } \\
\text { the CHX group. The higher level of tooth staining and calculus in the CHX group } \\
\text { meant that participants could have worked out which group they were in (or } \\
\text { that they were in the active mouthrinse group) and this could have affected } \\
\text { their oral health behaviours and hence the outcome. The direction of this po- } \\
\text { tential bias is unclear }\end{array}$ \\
\hline
\end{tabular}

Chlorhexidine mouthrinse as an adjunctive treatment for gingival health (Review)

Copyright $\odot 2017$ The Cochrane Collaboration. Published by John Wiley \& Sons, Ltd. 
de la Rosa 1988b (Continued)

Blinding of outcome as- High risk The authors report that the study is "double-blind". Quote: "Previous records sessment (detection bias) All outcomes were not available at subsequent examinations nor did examiners have any knowledge as to which treatment group a given subject belonged"

Comment: the authors report an increase in extrinsic tooth stain and supragingival calculus in the $\mathrm{CHX}$ group. The higher level of staining and calculus in the $\mathrm{CHX}$ group meant that the outcome assessor could not be adequately blinded and this could have affected the outcome assessment. The direction of this potential bias is unclear

Incomplete outcome data Unclear risk (attrition bias)

All outcomes
Numbers at baseline not reported, so attrition from baseline cannot be calculated
Selective reporting (re- High risk porting bias)
The SDs for the main outcome (mean gingivitis occurrence and severity) are not reported. The ancillary outcome (plaque) is mentioned but not fully reported possibly because the result was not statistically significant

Other bias Unclear risk No information is provided on intra- and interexaminer reproducibility.

The groups appear balanced for gingivitis at baseline. The authors state that the baseline scores demonstrate comparability of the test and control groups. However, the statistical significance of the differences is not reported

Eaton 1997

\section{Study characteristics}

\begin{tabular}{ll}
\hline Methods & Trial design: parallel ( 2 arms) \\
Location: suburban general dental practices in the South of England at Staines, Ripley, Lydd, Leigh on \\
Sea, and West Malling, UK \\
Number of centres: 5 \\
Study duration: recruitment period is not stated. Study duration and duration of rinsing was 3 months
\end{tabular}

Participants

Participants: healthy subjects, existing patients of the 5 general dental practices

Inclusion criteria: quote: "Inclusion criteria were: a willingness to participate in the study, a minimum of 16 natural teeth, the presence of chronic gingivitis but no pocket probing depths in excess of $4 \mathrm{~mm}$ or radiographic evidence of bone loss, no need for urgent treatment, between the ages of 18 and 65 years, in good health and a willingness to refrain from using any mouthrinses other than those provided in the study"

Exclusion criteria: quote: "Exclusion criteria were: acute gingivitis or other periodontal conditions, existing lesions of the oral mucosa, the receipt of antibiotics within 14 days of the baseline examination, history of diabetes, hepatitis $B$, tuberculosis or other infective or metabolic diseases, allergy to oral care products, the use of chlorhexidine or other antibacterial mouthrinses within three months of the start of the study, pregnancy or childbirth within the previous year, partial dentures, clinically unacceptable restorations, smoking more than 10 cigarettes per day and the receipt of any medication with a history of potential to alter gingival response"

Baseline gingivitis: (Modified GI) Gp A: mean CHX 0.56 (SD 0.4); Gp B: mean 0.54 (SD 0.4)

Age at baseline (years): Gp A: mean 34.8 (SD 11.4); Gp B: mean 34.9 (SD 12.7); range of 18-65

Gender: overall: male $41(34 \%)$, female $80(66 \%)$.

Number randomised: 121 (Gp A: 60; Gp B: 61). 24 subjects were at 4 practices each and 25 subjects were at the fifth practice

Number evaluated: 98 (Gp A: 48; Gp B: 50)

\section{Interventions Comparison: $\mathrm{CHX}$ versus control (placebo in aqueous solution)}

Gp A ( $n=60)$ : CHX (Peridex) 0.12\%, $15 \mathrm{~mL}$, twice daily, 30 seconds, 3 months 
Eaton 1997 (Continued)

Gp B ( $\mathrm{n}=61)$ : control, $15 \mathrm{~mL}$, twice daily, 30 seconds, 3 months

Prophylaxis at baseline: quote: "All were then (after baseline examination) given a dental prophylaxis... After initial baseline prophylaxis, patients were given no further prophylaxes during the study"

OHI: quote: "Patients were instructed to use only these oral hygiene items [medium, multitufted toothbrush and sodium fluoride containing toothpaste] throughout the study. They were asked to brush their teeth as normally"

Postrinsing instructions: not reported

Non-supervised rinsing

Timing of mouthrinsing in relation to toothbrushing: mouthrinsing after toothbrushing, after breakfast and last thing at night

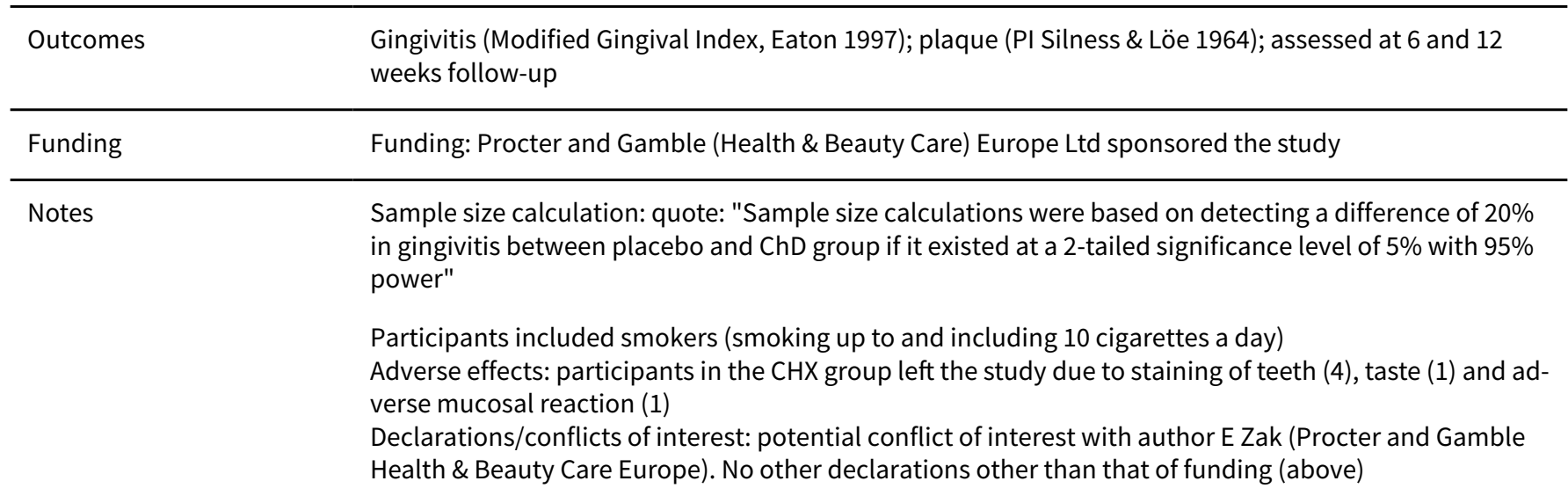

\section{Risk of bias}

\section{Bias Authors' judgement Support for judgement}

Random sequence genera- Low risk Quote: "mouthrinses....were dispensed on a randomised double-blind basis,
tion (selection bias) according to a random code"

Comment: adequate method of random sequence generation

\begin{tabular}{lll}
\hline $\begin{array}{l}\text { Allocation concealment } \\
\text { (selection bias) }\end{array}$ & Unclear risk & No information is provided \\
\hline $\begin{array}{l}\text { Blinding of participants } \\
\text { and personnel (perfor- } \\
\text { mance bias) }\end{array}$ & High risk & $\begin{array}{l}\text { The authors state that the study is double-blind. The CHX and placebo } \\
\text { mouthrinses were distributed in coded but otherwise indistinguishable con- } \\
\text { tainers }\end{array}$
\end{tabular}

Comment: tooth staining from the $\mathrm{CHX}$ was noted as a reason for loss to follow-up in the study and may have been visible to the participants in the test group. This meant that participants could have worked out which group they were in and this could have affected their oral health behaviours and hence the outcome. The direction of this potential bias is unclear

$\begin{array}{ll}\begin{array}{l}\text { Blinding of outcome as- } \\ \text { sessment (detection bias) }\end{array} & \text { High risk } \\ \text { All outcomes } & \text { The authors state that the study is double-blind } \\ & \begin{array}{l}\text { Comment: although the authors indicate that the clinical examiners were } \\ \text { blinded to the treatment groups, tooth staining from the CHX was noted as a } \\ \text { reason for loss to follow-up in the study. This meant that the outcome assessor } \\ \text { could not be adequately blinded and this could have affected the outcome as- } \\ \text { sessment. The direction of this potential bias is unclear }\end{array}\end{array}$

$\begin{array}{lll}\begin{array}{l}\text { Incomplete outcome data } \\ \text { (attrition bias) }\end{array} & \text { High risk } & \text { Attrition at } 12 \text { weeks 23/121 (19\%). Attrition by group: } 12 / 60 \text { (20\%) CHX, 11/61 } \\ \text { All outcomes } & (18 \%) \text { placebo. Reasons for loss to follow-up: CHX: } 5 \text { withdrawn because they } \\ & \text { had taken antibiotics during the study for non-oral reasons, a further } 6 \text { for fac- } \\ & \text { tors relating directly to the mouthrinses (4 toothstaining, } 1 \text { taste, } 1 \text { adverse }\end{array}$


mucosal reaction), 1 was unwilling/unable to continue in the study. Placebo: 8 withdrawn because they had taken antibiotics during the study for non-oral reasons, 3 for miscellaneous reasons including using a different mouthrinse, heavy smoking and unspecified unwillingness to continue

Comment: half of those lost from the $\mathrm{CHX}$ group were lost due to reasons directly related to using the $\mathrm{CHX}$ mouthrinse. Most losses in the placebo group were protocol violations. Although numerically the dropouts are similar, the reasons are different and may bias in favour of $\mathrm{CHX}$

Selective reporting (re- High risk
porting bias)

Adverse effects including tooth staining were reported in relation to withdrawals from the study only but are considered important outcomes and should have been reported for all participants where relevant

Other bias Low risk

Interexaminer $\mathrm{k}$ scores $0.78-0.85$ for $\mathrm{PI}$ and $0.73-0.94$ for the Modified Gingival Index. Examiners maintained $\mathrm{k}$ scores $0.51-0.90$ for $\mathrm{PI}$ and $0.73-1.00$ for the Modified Gingival Index during the 12 months required to complete the study. No mention of intraexaminer calibration

Groups are balanced for age, sex, gingivitis and plaque at baseline

Emling 1992

\section{Study characteristics}

Methods

Trial design: parallel ( 5 arms - 4 arms relevant to this review)

Location: assumed to be at School of Dental Medicine, University of Pennsylvania, Philadelphia, PA, USA

Number of centres: 1

Study duration: recruitment period is not stated. Study duration and duration of rinsing was 2 months
Participants: healthy subjects regardless of race or sex

Inclusion criteria: minimum of 20 crowned natural teeth; quote: "All subjects reported that they were free from any known reaction or sensitivity to any dentifrices, mouthrinses, or oral hygiene products. The female subjects entered into the study gave verbal assurance that they were not pregnant... All subjects agreed to refrain from any routine dental treatment or prophylaxis other than emergency procedures. They also agreed to use only the assigned treatments, and to comply with daily use as directed by the instructions given to them at the time of the product distribution"

Exclusion criteria: quote: "No subjects had orthodontic bands or diseases present of the hard tissues (osteomyelitis, fractures, cysts, odontomas, etc). There were no neoplastic growths in the oral cavity, advanced periodontal diseases, or diseases of the buccal mucosa, tongue, or floor of the mouth such as abscesses, ulcerations, hyperplasia, hypertrophy, or granulomas. None of the subjects entered into the study were currently using antibiotics or had used any antibiotics at least two weeks prior to the onset of the study"

Baseline gingivitis is reported separately for buccal and lingual surfaces

Baseline gingivitis for buccal surfaces: Gp A: mean 1.04 (0.59); Gp B: mean 0.92 (0.48); Gp C: mean 0.95 (0.62); Gp D: mean 1.24 (0.68); Gp E: mean $1.00(0.48)$

Baseline gingivitis for lingual surfaces: Gp A: mean 1.26 (0.56); Gp B: mean 1.18 (0.61); Gp C: mean 1.18 (0.55); Gp D: mean 1.26 (0.55); Gp E: mean1.07 (0.51)

Age at baseline (range): $18-60$

Gender: not stated. Subjects were accepted without reference to race or sex

Number randomised: 65 (assumed to be 13 in each group)

Number evaluated: not reported 
Emling 1992 (Continued)

Interventions

\section{Comparison: (Crest + CHX) versus (Rembrandt + CHX) versus (Crest + CHX until week 4 then Rem- brandt $+\mathrm{CHX}$ from week 4 to week 8)* versus (Crest + placebo) versus (Rembrandt + placebo)}

Gp A ( $n=13)$ : CHX (Peridex) $0.12 \%$ + Crest; amount not reported; frequency not reported; rinse time not reported, 8 weeks

Gp B ( $n=13):$ CHX (Peridex) $0.12 \%$ + Rembrandt; amount not reported; frequency not reported; rinse time not reported, duration not reported

${ }^{\star} \mathrm{Gp} \mathrm{C}(\mathrm{n}=13)$ : CHX (Peridex) $0.12 \%$ + Crest (baseline to week 4); CHX (Peridex) $0.12 \%+$ Rembrandt; amount not reported; frequency not reported; rinse time not reported; duration not reported. We excluded this arm from our data extraction, risk of bias and analysis

Gp D ( $n=13)$ : placebo + Crest; amount N/A; frequency not reported; rinse not reported; rinse time not reported; duration not reported

Gp E ( $n=13)$ : placebo + Rembrandt; amount N/A; frequency not reported; rinse not reported; rinse time not reported; duration not reported

Prophylaxis: not reported

OHI: quote: "At each examination time, subjects reported to the study having not brushed their teeth for a period of 10-12 hours"

Postrinsing instructions: not reported

Non-supervised rinsing

Timing of mouthrinsing in relation to toothbrushing: after toothbrushing

Outcomes Gingivitis (gingival inflammation: GI Löe \& Silness 1963); plaque (TQH,Turesky 1970); extrinsic tooth stain (area and intensity) (DI, Lobene 1968); supragingival calculus (Volpe-Manhold Calculus Index, Volpe-Manhold 1965); assessed at 2, 4, 6, and 8 weeks follow-up

\begin{tabular}{ll} 
Funding & Funding: not reported \\
\hline Notes & Sample size calculation: not reported \\
& Adverse effects: quote: "Eight subjects using Peridex complained of burning and/or irritation of the gin- \\
& giva or palatal mucosa. No other changes of the gingiva were observed during the study." Increases in \\
& stain intensity and area were noted in all CHX rinse groups during the study. Groups C and D had signifi- \\
& cant increases in mean calculus scores \\
& Declarations/conflicts of interest: none stated. First author works for International Dental Research Ltd, \\
& Moorestown, NJ, USA
\end{tabular}

\section{Risk of bias}

\begin{tabular}{lll}
\hline Bias & Authors' judgement & Support for judgement \\
\hline $\begin{array}{l}\text { Random sequence genera- } \\
\text { tion (selection bias) }\end{array}$ & Unclear risk & $\begin{array}{l}\text { Quote: "Subjects were randomly assigned to one of } 5 \text { groups.." } \\
\text { Comment: no description of the method of random sequence generation }\end{array}$ \\
\hline $\begin{array}{l}\text { Allocation concealment } \\
\text { (selection bias) }\end{array}$ & Unclear risk & No information is provided \\
\hline $\begin{array}{l}\text { Blinding of participants } \\
\text { and personnel (perfor- } \\
\text { mance bias) }\end{array}$ & High risk & $\begin{array}{l}\text { Quotes: "The study was conducted in a double-blind manner. Neither the sub- } \\
\text { jects nor the clinical examiners were aware of the product assignments at any } \\
\text { time during the study", and "The dentifrices and the mouthrinses were all in } \\
\text { similar packages labelled only with a subject's assigned number and the inves- } \\
\text { tigators emergency day and night phone numbers" }\end{array}$ \\
Increases in stain intensity and area were noted in all CHX rinse groups during \\
the study
\end{tabular}


Comment: The higher level of tooth staining in the $\mathrm{CHX}$ groups meant that participants could have worked out which group they were in (or that they were in 1 of the active mouthrinse groups) and this could have affected their oral health behaviours and hence the outcome. The direction of this potential bias is unclear

Blinding of outcome assessment (detection bias) All outcomes
High risk

Quotes: "The study was conducted in a double-blind manner. Neither the subjects nor the clinical examiners were aware of the product assignments at any time during the study", and "The dentifrices and the mouthrinses were all in similar packages labelled only with a subject's assigned number and the investigators emergency day and night phone numbers"

Increases in stain intensity and area were noted in all $\mathrm{CHX}$ rinse groups during the study

Comment: the higher level of tooth staining in the $\mathrm{CHX}$ groups meant that the outcome assessor could not be adequately blinded and this could have affected the outcome assessment. The direction of this potential bias is unclear

Incomplete outcome data Unclear risk No information is provided
(attrition bias)

All outcomes

Selective reporting (re- Low risk porting bias)

\begin{abstract}
All outcomes stated in the methods section are adequately reported in the results section. For gingivitis, plaque, stain intensity and stain area, means and SDs for buccal and lingual surfaces were combined for each group. Then, $\mathrm{CHX}$ + Crest and $\mathrm{CHX}+$ Rembrandt groups were combined into a $\mathrm{CHX}+$ toothpaste group and placebo + Crest and placebo + Rembrandt were combined into a placebo + toothpaste group to be included in meta-analyses
\end{abstract}

\begin{tabular}{ll}
\hline Other bias & $\begin{array}{l}\text { No information is provided on number of examiners or intra- and interexamin- } \\
\text { er reproducibility }\end{array}$ \\
& $\begin{array}{l}\text { Baseline } \mathrm{GI}, \text { plaque, calculus and stain indices were not statistically significant- } \\
\text { ly different between the groups at baseline }\end{array}$
\end{tabular}

Ernst 2005

\title{
Study characteristics
}

Methods

Trial design: parallel ( 3 arms - 2 arms are relevant to this review)

Location: Department for Operative Dentistry, Johannes Gutenberg University of Mainz, Germany

Number of centres: 1

Study duration: recruitment period not stated. Study duration and duration of rinsing was 4 weeks

Participants

Participants: quotes: "soldiers stationed in Mainz, Germany" and "students from the dental clinic of Johannes Gutenberg University Mainz, Germany"

Inclusion criteria: quote: "Each volunteer had gingivitis or slight periodontitis"

Exclusion criteria: quote: "General exclusion criteria were systemic diseases and long-term medications"

Baseline gingivitis (GI, Löe \& Silness): Gp A: mean 1.21 (SD 0.68); Gp B: mean 1.11 (SD 0.88); Gp C: mean 1.09 (SD 0.71) 
Ernst 2005 (Continued)

Age of completers (years): Gp A: mean 28.4 (SD 8.5); Gp B: mean 32.2 (SD 12.2); Gp C: mean 31.3 years

(SD 9.5). Overall age range 18-58. Gp A: range 18-50; Gp B: range 22-58; Gp C: range 22-54

Gender: not reported

Number randomised: 101 (not reported by group)

Number evaluated: 90 (Gp A: 33; Gp B: 29; Gp C: 28)

Interventions

Comparison: $\mathrm{CHX}$ versus Hexetidine* versus placebo

Gp A (completers $\mathrm{n}=33$ ): CHX 0.1\%, $15 \mathrm{~mL}$, twice daily, 30 seconds, 4 weeks

*Gp B (completers $n=29$ ): Hexetidine $0.1 \%, 15 \mathrm{~mL}$, twice daily, 30 seconds, 4 weeks. We excluded this arm from the risk of bias and analysis

Gp C (completers $n=28)$ : placebo, $15 \mathrm{~mL}$, twice daily, 30 seconds, 4 weeks

Prophylaxis at baseline: quote: "Each subject's teeth were professionally cleaned afterwards"

OHI: quote: "The subjects were given detailed instructions on oral hygiene. Each subject received a new toothbrush (Oral B, P35, Gilette/Oral B) and several trial packages of a standardized toothpaste (Emex, GABA). The subjects were asked to brush their teeth with the new toothbrush and toothpaste after breakfast and dinner each day"

Non-supervised rinsing

Timing of mouthrinising in relation to toothbrushing: after toothbrushing

Outcomes

Gingivitis (gingival inflammation: GI Löe \& Silness 1967; gingival bleeding: Bleeding Index, van der Weijen 1994); plaque (Approximal Plaque Index, Lange 1977); extrinsic tooth stain (Discolouration Index, no reference provided but as described it appears to be the severity aspect of the Discolouration Index (Lobene 1968)); assessed at 2 weeks (GI and DI) and at 4 weeks (all indices). Volunteers were questioned about changes to taste, oral sensitivity, taste of mouthrinse, and about their opinion of the mouthrinse (the latter only asked after 4 weeks); assessed at 4 weeks follow-up

\begin{tabular}{ll}
\hline Funding & Not reported \\
\hline Notes & Sample size calculation: not reported
\end{tabular}

Adverse effects: quote: "A total of 28 of 90 subjects reported obvious changes in their sense of taste after using the mouthrinse. The number of subjects using mouthrinse $B$ reported the most incidences of a change in their sense of taste. A total of 18 of 29 subjects (62.1\%) complained about changes in their sense of taste. Only 8 of 33 subjects (24.2\%) using mouthrinse A reported a change in the sense of taste. In the group using mouthrinse C, 2 out of 28 subjects (7.1\%) complained about obvious changes in their sense of taste... A relatively high number of subjects using mouthrinse B complained about oral mucosa sensitivities. Two subjects out of this groups showed such severe changes in terms of intensive red, inflamed areas at the intermediate check-up that mouthrinse use was discontinued immediately... In group 1 (using mouthrinse A), 5 of 33 subjects (15\%) showed sensitivities of the oral mucosa... A total of $24 \%$ of the subjects in group 1 suffered from changes in their sense of taste." Tooth staining was significantly higher in the $\mathrm{CHX}$ group compared to the 2 other groups

Smokers: Gp A 17/33 (51.5\%); Gp B 12/29 (41.4\%); Gp C 8/28 (28.6\%)

Declarations/conflicts of interest: none stated

\section{Risk of bias}

\begin{tabular}{lll}
\hline Bias & Authors' judgement & Support for judgement \\
\hline $\begin{array}{l}\text { Random sequence genera- } \\
\text { tion (selection bias) }\end{array}$ & Unclear risk & $\begin{array}{l}\text { Quotes: "...divided randomly into two groups" and "the solutions were handed } \\
\text { out randomly to each subject" }\end{array}$ \\
\hline
\end{tabular}


Allocation concealment Low risk (selection bias)
Quote: "All mouthrinses were coded and placed into identical bottles. The bottles were labelled A, B, or C by the pharmacy at the Universtiy Clinic Mainz, Mainz, Germany. The solutions were handed out randomly to each subject"

Comment: the details suggest that concealment of allocation sequence was adequate
Blinding of participants High risk Quotes: "Double-blind.." and "All mouthrinses were coded and placed into and personnel (performance bias)

All outcomes identical bottles. The bottles were labelled A, B or C by the pharmacy at the University Clinic Mainz, Mainz, Germany.. each solution contained the same application and dosage instructions. The bottles were not decoded until after all the follow-up examinations and final statistical analyses had been completed." The placebo contained propyleneglycol, glycerol, gentian-tincture, pigment E 124 and purified water

Comment: the $\mathrm{CHX}$ group developed more tooth staining than the placebo group wwhich meant that participants could have worked out which group they were in (or that they were in 1 of the active mouthrinse groups) and this could have affected their oral health behaviours and hence the outcome. The direction of this potential bias is unclear

Blinding of outcome as- High risk sessment (detection bias) All outcomes
Quotes: "Double-blind.." and "All mouthrinses were coded and placed into identical bottles. The bottles were labelled A, B or C by the pharmacy at the University Clinic Mainz, Germany.. each solution contained the same application and dosage instructions. The bottles were not decoded until after all the follow-up examinations and final statistical analyses had been completed... All exams were done by clinicians at the Department of Operative Dentistry, Johannes Gutenberg University"

Comment: the $\mathrm{CHX}$ group developed more tooth staining than the placebo group which meant that the outcome assessor could not be adequately blinded and this could have affected the outcome assessment. The direction of this potential bias is unclear

Incomplete outcome data Unclear risk Attrition at 5 weeks 11/101 (10.9\%). Not broken down by group

(attrition bias)

All outcomes

Reasons for losses: non-compliance

\begin{tabular}{lll}
$\begin{array}{l}\text { Selective reporting (re- } \\
\text { porting bias) }\end{array}$ & Low risk & $\begin{array}{l}\text { All outcomes stated in the methods section are adequately reported in the re- } \\
\text { sults section. Adverse effects including tooth staining are reported in detail }\end{array}$ \\
\hline Other bias & Unclear risk & $\begin{array}{l}\text { No information is provided on number of examiners, intra- and interexaminer } \\
\text { reproducibility or training of examiners }\end{array}$ \\
& $\begin{array}{l}\text { The mean scores for GI, Bleeding Index, Approximal Plaque Index and DI ap- } \\
\text { pear similar at baseline. Mean age of completers and age range appear similar. } \\
\text { There were a higher proportion of cigarette smokers in Gp A compared to Gp C. } \\
\text { Gender is not reported }\end{array}$
\end{tabular}

\title{
Faveri 2006
}

\section{Study characteristics}

\author{
Methods \\ Trial design: parallel (2 arms) \\ Location: Periodontal Clinic of Guarulhos University, Guarulhos, Sao Paulo, Brazil
}


Faveri 2006 (Continued)

Number of centres: assumed to be 1

Study duration: the recruitment period is not stated. Study duration is 84 days ( -21 days to +63 days post-therapy. Duration of rinsing was 63 days (-21 days to +42 days post-therapy)

Participants

Participants: adults referred to the periodontal clinic of Guarulhos University, Brazil

Inclusion criteria: quote: "The study included subjects $>30$ years of age with at least 15 teeth and a minimum of six teeth with at least one site with PD between 5 and $7 \mathrm{~mm}$ and CAL between 5 and $10 \mathrm{~mm}$ "

Exclusion criteria: quote: "The exclusion criteria were as follows: previous periodontal therapy, pregnancy, nursing, smokers, any systemic condition that could affect the progression of periodontal disease or that required antibiotic coverage for routine dental therapy, allergy to $\mathrm{CHX}$ and antibiotic therapy in the previous 6 months"

Baseline gingivitis: (gingival gleeding yes/no), Gp A: mean 33.02 (+/-11.37); Gp B: mean 40.17 (+/-19.46)

Age at baseline (years): range not stated. Gp A: mean 48.5 (+/-8.8); Gp B: mean 42.1 (+/-6.5)

Gender: overall: male 13 (45\%); female $16(55 \%)$. Gp A: male 5 (36\%), female 9 (64\%); Gp B: male 8 (53\%), female 7 (47\%)

Number randomised: 30 (Gp A: 15; Gp B: 15)

Number evaluated: 29 (Gp A: 14; Gp B: 15)

\section{Comparison: (CHX +SRP) versus (placebo control + SRP)}

Gp A $(n=14): C H X 0.12 \%+S R P, 15 \mathrm{~mL}$, twice daily, 1 min, 63 days

Gp B ( $n=15)$ : placebo control (composition not described) + SRP, $15 \mathrm{~mL}$, twice daily, $1 \mathrm{~min}, 63$ days

Prophylaxis at baseline: quote: "During the initial phase, subjects received... full-mouth supragingival scaling"

OHI: quote: "During the initial phase, subjects received... instruction in proper home-care techniques." All participants received scaling and root planing "completed in a maximum of six appointments lasting approximately $1 \mathrm{~h}$ each." The treatment was completed in 21 days. The $\mathrm{CHX}$ rinsing began with the SRP and continued for 42 days after the end of this therapy

Non-supervised rinsing

Timing of mouthrinsing in relation to toothbrushing: after toothbrushing

Outcomes Gingivitis (gingival bleeding 0/1, BOP 0/1); plaque (plaque accumulation 0/1); suppuration (0/1); assessed at 42 and 63 days postSRP

Probing depth ( $\mathrm{mm})$; CAL $(\mathrm{mm})$ assessed and microbiological monitoring also carried out (not relevant to this review)

Funding Q Quote: "This study was supported by Guarulhos University, Guarulhos, SP-Brazil and FAPESP"

\section{Notes}

Sample size calculation: quote: "The sample size calculation determined that 14 subjects per group would provide an $80 \%$ power to detect a true difference of $1.0 \mathrm{~mm}$ between test and placebo using probing pocket depth reduction in pockets $>/ 6 \mathrm{~mm}$ as the primary outcome variable. This calculation was based on a two-tailed comparison of $\alpha=0.05$ "

Adverse effects: quote: "No severe adverse effects were reported by any of the subjects"

Non-smokers

Declarations/conflicts of interest: none stated 
Faveri 2006 (Continued)

Risk of bias

\begin{tabular}{lll}
\hline Bias & Authors' judgement & Support for judgement \\
\hline $\begin{array}{ll}\text { Random sequence genera- } \\
\text { tion (selection bias) }\end{array}$ & Low risk & $\begin{array}{l}\text { Quote: "During the enrollment visit, each subject was given a code number, } \\
\text { and a computer-generated table was used to have them randomly assigned to } \\
\text { receive one of the two proposed treatments" }\end{array}$ \\
& $\begin{array}{l}\text { Comment: adequate method of random sequence generation } \\
\end{array}$
\end{tabular}

\begin{tabular}{ll}
\hline $\begin{array}{l}\text { Allocation concealment } \\
\text { (selection bias) }\end{array}$ & Quigh risk \\
& $\begin{array}{l}\text { and a computer-generated table was used to have them randomly assigned to } \\
\text { receive one of the two proposed treatments. The coordinator of the study as- } \\
\text { signed participants to their groups" }\end{array}$
\end{tabular}

Comment: it is likely that the study co-ordinator was aware of the upcoming assignments when randomising the subjects

$\begin{array}{ll}\begin{array}{l}\text { Blinding of participants } \\ \text { and personnel (perfor- }\end{array} & \begin{array}{l}\text { The study is described as single-blind. The university pharmacy prepared the } \\ \text { placebo and } \mathrm{CHX} \text { rinses and packaged them in opaque plastic tubes. The study } \\ \text { mance bias) }\end{array} \\ \text { All outcomes } & \begin{array}{l}\text { co-ordinator marked the code number of each subject on a set of } 9 \text { tubes. The } \\ \text { co-ordinator gave the coded tubes to the } 2 \text { examiners who at no time during }\end{array} \\ & \text { the study had access to information about the contents of the tubes or the as- } \\ & \text { signment of subjects to groups. Quote: "All study personnel, including the bio- } \\ \text { statistician and participants, were blinded to treatment assignment for the du- } \\ \text { ration of the study" }\end{array}$

Comment: blinding was unlikely to be maintained if there was more staining and other minor adverse effects in the $\mathrm{CHX}$ group (it is stated that no serious adverse effects were reported by any of the subjects). However, as these outcomes were not reported, it is unclear whether blinding was maintained

\begin{tabular}{|c|c|c|}
\hline $\begin{array}{l}\text { Blinding of outcome as- } \\
\text { sessment (detection bias) } \\
\text { All outcomes }\end{array}$ & Unclear risk & $\begin{array}{l}\text { The study is described as single-blind. The university pharmacy prepared the } \\
\text { placebo and CHX rinses and packaged them in opaque plastic tubes. The study } \\
\text { co-ordinator marked the code number of each subject on a set of } 9 \text { tubes. The } \\
\text { co-ordinator gave the coded tubes to the } 2 \text { examiners who at no time during } \\
\text { the study had access to information about the contents of the tubes or the as- } \\
\text { signment of subjects to groups. Quotes: "All study personnel, including the } \\
\text { biostatistician and participants, were blinded to treatment assignment for } \\
\text { the duration of the study" and "One examiner performed all clinical measure- } \\
\text { ments... and treatment was performed by the second examiner" }\end{array}$ \\
\hline
\end{tabular}

Comment: blinding was unlikely to be maintained if there was more staining and other adverse effects in the $\mathrm{CHX}$ group (it is stated that no serious adverse effects were reported by any of the subjects). However, as these outcomes were not reported, it is unclear whether blinding was maintained

Incomplete outcome data Low risk (attrition bias)

All outcomes
Attrition at 12 weeks: $1 / 30$ (3.3\%). 1 subject lost from the $\mathrm{CHX}$ group because they had taken an antibiotic during the course of the study

$\begin{array}{ll}\begin{array}{l}\text { Selective reporting (re- } \\ \text { porting bias) }\end{array} & \text { High risk } \\ & \begin{array}{l}\text { All outcomes are reported graphically with bars representing a measure of } \\ \text { variance in the graphs. We obtained additional outcome data from the first } \\ \text { author. Toothstaining is considered to be an important side effect of } \mathrm{CHX} \\ \text { mouthrinsing but this is not reported }\end{array}\end{array}$

Other bias

Low risk

Quote: "The clinical monitoring was performed by two trained and calibrated examiners.. One examiner performed all clinical measurements... and treatment was performed by the second examiner" 
The examiners were calibrated to provide reproducible measurements for probing depth and CAL. Inter- and intraexaminer variability is reported for probing depth and CAL measurements

"The demographic and clinical baseline characteristics were similar between the two groups $(P>0.05) "$

\section{Study characteristics}

Methods

Trial design: parallel ( 3 arms - 2 arms relevant to this review)

Location: Periodontal Clinic of Guarulhos University, Guarulhos, Sao Paulo, Brazil

Number of centres: 1

Study duration: recruitment period is not stated. Study duration was 6 months and 21 days. Duration of rinsing was 63 days

Participants

Participants: quote: "population referred to the Periodontal Clinic of Guarulhos University"

Inclusion criteria: quote: "subjects with untreated previously periodontal disease... good general health and were diagnosed with generalized chronic periodontitis based on the current classification of the American Academy of Periodontology. The inclusion criteria were as follows: $>30$ years of age, at least 15 teeth, minimum of six teeth with at least one interproximal site with PD between 5 and $7 \mathrm{~mm}$ and CAL between 5 and $10 \mathrm{~mm}$, at least $30 \%$ of the sites with PD and CAL $>/ 5 \mathrm{~mm}$ and presence of bleeding on probing (BOP)"

Exclusion criteria: quote: "The exclusion criteria were as follows: previous subgingival periodontal therapy, smoking, pregnancy, nursing, systemic diseases that could affect the progression of periodontal disease (e.g. diabetes and immunological disorders), long-term administration of anti-inflammatory medication, need for antibiotic coverage for routine dental therapy, antibiotic therapy in the previous 6 months and allergy to $\mathrm{CHX"}$

Baseline gingivitis: (gingival bleeding) Gp A: mean 29.6 (+/- 13.8); Gp B: mean 34.9 (+/- 19.4); Gp C: mean $40.3(+/-22.5)$

Age at baseline (years): range not stated. Gp A: mean 45.1 (+/- 9.6); Gp B: mean 39.6 (+/- 6.1); Gp C: mean $42.5(+/-7.3)$

Gender: overall: male 24 (40\%), female $36(60 \%)$. Gp A: male 8 (40\%), female $12(60 \%)$; Gp B: male 7 (35\%), female $13(65 \%)$; Gp C: male 9 (45\%), female $11(55 \%)$

Number randomised: 60 (Gp A: 20; Gp B: 20; Gp C: 20)

Number evaluated: 60 (Gp A: 20; Gp B: 20; Gp C: 20)

Interventions

\section{Comparison: (SRP + CHX) versus (SRP + placebo) versus (SRP, professional plaque control and placebo)*}

All participants received full-mouth SRP under local anaesthesia in 4-6 appointments of 1 hour each. SRP was completed 21 days after baseline. Participants started rinsing at the beginning of the study and continued rinsing for 42 days after the end of the SRP

$\mathrm{Gp} \mathrm{A}(\mathrm{n}=20): \mathrm{SRP}+\mathrm{CHX} 0.12 \%, 15 \mathrm{~mL}$, twice daily, $1 \mathrm{~min}, 63$ days

Gp B ( $n=20): S R P$ + placebo, $15 \mathrm{~mL}$, twice daily, 1 min, 63 days

${ }^{*} \mathrm{Gp} \mathrm{C}(\mathrm{n}=20)$ : SRP, professional plaque control and placebo, $15 \mathrm{~mL}$, twice daily, $1 \mathrm{~min}, 63$ days (data not included in the review). We excluded this arm from our risk of bias and analysis 
Feres 2009 (Continued)

Prophylaxis at baseline: quote: "During the initial phase, all subjects received... full-mouth supragingival scaling"

OHI: quote: "During the initial phase, all subjects received instruction on proper homecare techniques"

Non-supervised rinsing

Timing of mouthrinsing in relation to toothbrushing: after toothbrushing

Outcomes

Outcomes measured at 2 and 6 months post-therapy (i.e. first follow-up examination occurred approx 20 days after rinsing had ceased at approx 83 days after baseline)

Gingivitis: (gingival bleeding (0/1); BOP (0/1)); visible plaque (0/1) measured at 2 and 6 months posttherapy

Suppuration (0/1); PD (mm); CAL (mm); also microbiological monitoring; assessed at 2 and 6 months post-therapy (not relevant to this review)

Funding

This study was supported in part by research grants from the state of Sao Paulo research foundation and FOGARTY from the National Institutes of Health (NIH), USA

Notes

Sample size calculation: quote: "The ideal sample size to assure adequate power for this clinical trial was calculated considering differences of at least $1 \mathrm{~mm}$ between groups for clinical attachment level $(\mathrm{CAL})$ in initially deep periodontal sites [probing depth $(\mathrm{PD})>/ 7 \mathrm{~mm}$ ]... Based on these calculations, it was defined that 16 subjects per group would be necessary to provide an $80 \%$ power with an [alpha] of $0.05 "$

Adverse effects: quote: "No severe adverse effects were reported by any of the subjects. Two subjects from the $\mathrm{CHX}$ group reported adverse events during the study, such as tooth staining and an unpleasant taste"

Non-smokers

Declarations/conflicts of interest: none stated

Notes: intention-to treat analysis. The last available recordings were carried forward to represent all subsequent time points of evaluation. Data from professional plaque control group are not included in the review

\section{Risk of bias}

Bias Authors' judgement Support for judgement

Random sequence genera- Low risk tion (selection bias)
Quote: "Each subject was given a code number at the enrolment visit and the study coordinators used a computer-generated table (in blocks of three) to randomly allocate them to one of three therapeutic groups"

Comment: adequate method of random sequence generation
Allocation concealment Low risk (selection bias)
Quotes: "Each subject was given a code number at the enrolment visit and the study coordinators used a computer-generated table (in blocks of three) to randomly allocate them to one of three therapeutic groups." The mouthrinses were prepared and dispensed in opaque plastic tubes by the Guarulhos university pharmacy. "The study coordinators marked the code number of each subject on a set of 9 tubes according to the therapy assigned"

Additional information provided by the study authors: "Allocation concealment was assured by placing the numbered bottles in indistinguishable plastic bags with the same numbering. The numbered bags with the medication/placebo were given to the therapists by a clinical assistant, following the sequence of numbers from 1 to 60 . This assistant and the clinicians had no access to the randomization list, assuring the allocation concealment. The study 
Feres 2009 (Continued) coordinators had access to the list but did not participate on the clinical treatment/examination of the patients"

Comment: the details suggest that concealment of allocation sequence was adequate

Blinding of participants Unclear risk and personnel (performance bias)

All outcomes

$\mathrm{CHX}$ and placebo rinse was packaged in opaque plastic tubes with the patients code number written on the tubes. "The examining researchers had no access to information about the contents of the tubes or assignment of subjects to the three therapies. All study personnel, including the biostatistician and participants were not aware of the treatment assignments for the duration of the study"

2 subjects from the $\mathrm{CHX}$ group reported adverse events during the study, such as tooth staining and an unpleasant taste

Comment: blinding of all participants is not possible in this study because of differences between the interventions (subjects in the professional plaque control group attended the clinic twice a week and the others did not) although the $\mathrm{CHX}$ and placebo groups could be considered blind. The presence of noticeable tooth staining was noted by 2 of the participants. It is not clear if other participants in the CHX group noticed increased tooth staining

Blinding of outcome as- Unclear risk sessment (detection bias) All outcomes

Quotes: "The examining researchers had no access to information about the contents of the tubes or assignment of subjects to the three therapies. All study personnel, including the biostatistician and participants were not aware of the treatment assignments for the duration of the study" and "One examiner carried out all clinical measurements in a given subject and treatment was performed by the second clinician. Thus, the monitoring clinician was masked to the treatment protocol"

Comment: the presence of noticeable tooth staining was noted by 2 of the participants and would also have been visible to the outcome assessors. As tooth staining was not measured in the course of the study, it is not clear if other participants in the $\mathrm{CHX}$ group had increased tooth staining that would have been visible to the outcome assessors

Intention-to-treat analysis was carried out using last available recordings for those lost to follow-up. Therefore all subjects who entered the study were included in the analysis at all time points

Attrition (all study arms) 11/60 (18.3\%). Dropouts by group: CHX 5/20 (25\%), placebo 3/20 (15\%). Reasons for loss to follow-up: CHX: 2 moved to another city, 1 got pregnant and 2 could not be contacted; placebo: 3 could not be contacted

Comment: dropouts are high particularly in the $\mathrm{CHX}$ group. Reasons for losses do not appear to be related to the intervention

Selective reporting (re- High risk
porting bias)

All outcomes stated in the methods section are adequately reported in the results section. However, tooth staining is considered to be an important side effect of $\mathrm{CHX}$ mouthrinsing and apart from being self-reported by 2 participants, this is not otherwise reported

Other bias Unclear risk

\author{
The 2 examining researchers were calibrated for the periodontal PD and CAL \\ measurements only
}

Quote: "No statistically significant differences were observed among groups for any parameter at baseline" 


\section{Study characteristics}

Methods

Trial design: parallel ( 6 arms) ( $2 \times 3$ factorial design; 2 arms relevant to this review are described here)

Location: Periodontal Clinic of Guarulhos University, Brazil

Number of centres: 1

Study duration: the recruitment period is not stated, however, the study duration was 1 year and the duration of rinsing was 2 months

Participants

Participants: healthy subjects with untreated generalized chronic periodontitis

Inclusion criteria: at least 30 years old, at least 6 teeth, at least 1 site with PD and CAL $4 \mathrm{~mm}$ or more, BOP

Exclusion criteria: previous subgingival periodontal therapy, pregnant or breastfeeding, smoking, systemic disease that could affect progression of periodontal disease, long-term use of anti-inflammatory medication, need for antibiotic premedication for routine dental therapy, use of antibiotic therapy in previous 6 months, allergy to metronidazole, amoxicillin or $\mathrm{CHX}$

Baseline gingivitis: (\% of sites with BOP) overall mean for SRP group (1): 70.6 (SD 23.8); within group (1) $\mathrm{CHX}+\mathrm{SRP} 69.2 \pm 26.5, \mathrm{SRP} 72.0 \pm 21.4$

Age at baseline (years): mean 45.8 (SD 8.54) from Table 1 (mean of data reported in Table S1 is 43.3); CHX: $41.6 \pm 7.2$, placebo: $45.2 \pm 10.1$

Gender: overall: male 12 (30\%), female 28 (70\%); CHX: male: 7, female: 13; placebo: male: 5, female: 15 Number randomised: 40 (Gp A: 20; Gp B: 20)

Number evaluated: 40 (Gp A: 20; Gp B: 20) at 3 months; ITT analysis (last observation carried forward) used to account for dropouts at 6 and 12 months (not reported by $\mathrm{CHX/placebo)}$

Interventions

\section{Comparison: (1) SRP versus (2) SRP + metronidazole versus (3) root planing + metronidazole + amoxicillin}

Each arm was further randomised to receive $\mathrm{CHX}$ rinse or placebo rinse. Only the first arm (1) was eligible for inclusion in this review

Gp A ( $n=20):$ CHX 0.12\% + SRP, $15 \mathrm{~mL}$, twice daily, 1 min, 2 months

Gp B $(n=20):$ placebo + SRP as above

Prophylaxis at baseline: SRP (4 to 61 -hour sessions under local anaesthesia over 14 days) and "all subjects received periodontal maintenance at the three post-treatment monitoring visits"

OHI: "During the initial phase, all subjects received instruction on proper home-care techniques and were given the same dentifrice (Colgate Total; Colgate Palmolive Co, São Bernardo do Campo, SP, Brazil) to use during the study period"

Non-supervised rinsing

Timing of mouthrinsing in relation to toothbrushing: "Subjects were instructed to rinse in the morning, $30 \mathrm{~min}$ after breakfast and toothbrushing, and also at night $30 \mathrm{~min}$ after toothbrushing"

Outcomes

Gingivitis (BOP yes/no, gingival bleeding yes/no); plaque (\% sites with accumulation); periodontitis (probing depth, CAL); and suppuration assessed at 3, 6 and 12 months

Because participants received periodontal maintenance at 3, 6 and 12 months, the 3-month data are considered to be the only data relevant to this review 
Feres 2012 (Continued)

Funding Q Quote: "This study was supported by Research Grant \#07/55291-9 from Fundação de Amparo à Pesquisa do Estado de São Paulo (FAPESP, Brazil)"

Notes

Sample size calculation: accounting for $15 \%$ attrition, 39 participants were needed per group (the initial 3 groups before further division into $\mathrm{CHX} /$ placebo) in order to detect a difference of 4 sites with probing depth $5 \mathrm{~mm}$ or greater at $90 \%$ power and $5 \%$ significance (unclear whether the study is powered to detect a difference between $\mathrm{CHX} /$ placebo within each of the $3 \mathrm{arms}$ )

Adverse effects: adverse effects reported appear to be related to the metronidazole and amoxicillin, but there were no statistically significant differences between any of the 3 groups

Declarations/conflicts of interest: quote: "The authors declare that they have no conflict of interests"

Data for the SRP+CHX and SRP groups within group (1) were obtained from the study authors

\section{Risk of bias}

Bias Authors' judgement Support for judgement

Random sequence genera- Unclear risk tion (selection bias)

Quotes: "each of the selected subjects was given a code number during the enrolment visit and the study coordinators (M.Fe. and L.C.F.) used a computer-generated table to allocate them to one of the three therapeutic groups" and "A second randomization was performed to allocate half of the subjects in each group to rinse with...CHX or placebo"

Comment: second randomisation not adequately described

Allocation concealment $\quad$ Unclear risk Comment: not described
(selection bias)

Blinding of participants Unclear risk and personnel (performance bias)

All outcomes

rinsing for 2 months, therefore participants could have worked out which
group they were in and this could have affected their oral health behaviours
and hence the outcome. However, tooth staining is not reported and there is
not enough information upon which to base a judgement

\begin{tabular}{|c|c|c|}
\hline $\begin{array}{l}\text { Blinding of outcome as- } \\
\text { sessment (detection bias) }\end{array}$ & Unclear risk & $\begin{array}{l}\text { Quotes: "double-blind" and "Masking of } \mathrm{CHX} \text { rinsing may have been hampered } \\
\text { by tooth staining" }\end{array}$ \\
\hline & & $\begin{array}{l}\text { Comment: tooth staining is likely to have been noticeable in the } \mathrm{CHX} \text { groups } \\
\text { at } 3 \text { months follow-up and in this situation the outcome assessor could have } \\
\text { worked out which individuals used } \mathrm{CHX} \text { and not be adequately blinded. How- } \\
\text { ever, tooth staining is not reported and there is not enough information upon } \\
\text { which to base a judgement }\end{array}$ \\
\hline
\end{tabular}

\begin{tabular}{lll}
\hline $\begin{array}{l}\text { Incomplete outcome data } \\
\text { (attrition bias) }\end{array}$ & Low risk & $\begin{array}{l}\text { All randomised participants were included in the analyses. ITT analysis (last } \\
\text { observation carried forward) used to account for dropouts at } 6 \text { and } 12 \text { months } \\
\text { (not reported by CHX/placebo) }\end{array}$ \\
All outcomes & (n)
\end{tabular}

All outcomes

Selective reporting (re- High risk porting bias)

Tooth staining is considered to be an important side effect of $\mathrm{CHX}$ mouthrinsing but this is not reported. Only the primary outcome (PD) was reported by $\mathrm{CHX} /$ placebo in the published article

\begin{tabular}{ll}
\hline Other bias & Calibration carried out with high Kappa scores for inter- and intraexaminer \\
& $\begin{array}{l}\text { agreement }(>92 \%) \text {, but this may have only been for the primary outcomes of } \\
\text { the study, which were for periodontitis (PD and CAL) }\end{array}$
\end{tabular}




\section{Study characteristics}

Methods
Location: assumed to be University of Kentucky Medical Center, USA
Number of centres: 1
Study duration: recruitment period not stated. Study duration is 90 days. Duration of rinsing was 60
days
Participants: quote: "patients undergoing assessment for bone marrow transplantation"
Inclusion criteria: quote: "patients undergoing assessment for bone marrow transplantation... patients
with dentition"
Exclusion criteria: quote: "Two patients were not included in evaluation because, for medical reasons,
they did not undergo bone marrow transplantation. These excluded patients were similar in all other
aspects to the 33 patients who were evaluated"
Baseline gingivitis: not reported
Age at baseline (years): Gp A: mean 25.3 $\pm 3.3 ;$ Gp B: mean 27.1 \pm 3.9. Gp A: range 5-48; Gp B: range 5-51
Gender: not reported
Number randomised: 35 (Gp A: 18; Gp B: 17)
Number evaluated: assumed 33 (Gp A: 16; Gp B: 17 ) but this is unclear. See note under 'Attrition bias'

Comparison: $\mathrm{CHX}$ versus placebo control
Interventions
$\mathrm{Gp} \mathrm{B} \mathrm{(} \mathrm{n}=16): \mathrm{CHX} 0.12 \%, 15 \mathrm{~mL}, 3$ times daily, 30 seconds, 60 days
Prophylaxis at baseline: quote: "Necessary dental care, including dental cleaning... was implemented
before the initiation of chemoradiotherapy"
OHI: none, usual but with foam rubber toothbrush substitute and saline solution irrigations
Partially supervised rinsing. Quote: "Supervised by the nursing staff of the transplant unit for the dura-
tion of the patients' hospitalization (usually 35-45 days). After discharge, patients continued this regi-
men for the remainder of the 60 -day treatment period"
Timing of mouthrinsing in relation to toothbrushing: after toothbrushing (with a foam rubber tooth-
brush)

Outcomes Gingivitis (gingival inflammation: PMGI, de la Rosa 1976); plaque (TQH, Turesky 1970); extrinsic tooth stain (Meckel's stain grading method, Lang 1977) assessed at 33, 60, and 90 days follow-up

Mucositis, oral streptococci and candida are also reported but are not relevant to this review

Funding Quote: "This study was supported by funds from the University of Kentucky, the Procter \& Gamble Co, and the Veterans Administration"

Notes

Sample size calculation: not reported

Adverse effects: quote: "Extrinsic dental staining was similar throughout the study for both the chlorhexidine and control groups. There was a modest, although not significant, increase in dental staining noted in both groups during the treatment" 
Ferretti 1987 (Continued)

Declarations/conflicts of interest: none stated

\section{Risk of bias}

Bias Authors' judgement Support for judgement

Random sequence genera- Unclear risk tion (selection bias)

\begin{tabular}{lll}
\hline $\begin{array}{l}\text { Allocation concealment } \\
\text { (selection bias) }\end{array}$ & Unclear risk & No information is provided
\end{tabular}

Blinding of participants Low risk and personnel (performance bias)

All outcomes
Quote: "..in a double-blind fashion." The placebo was identical in composition to the $\mathrm{CHX}$ only without the $\mathrm{CHX}$. Rinsing was supervised by the nursing staff. Blinding of the nursing staff although likely is not explicitly stated

Comment: there was a modest although not significant increase in dental staining noted in both groups during the treatment

\section{Blinding of outcome as- High risk} sessment (detection bias) All outcomes

\section{Quotes: "..in a double-blind fashion" and "All clinical indexes were scored by} the same examiner"

Comment: there was a modest although not significant increase in dental staining noted in both groups during the treatment. The incidence of oral candida and mucositis is markedly lower in the $\mathrm{CHX}$ group which may have affected blinding by potentially alerting the examiner to the group assignment of the subjects Incomplete outcome data High risk
(attrition bias)

All outcomes
According to Table 2: patient characteristics, 35 patients were randomised (18 $\mathrm{CHX}$ and 17 control) and 33 were evaluated ( $16 \mathrm{CHX}$ and 17 control). 2 participants were lost from the $\mathrm{CHX}$ group because they did not undergo bone marrow transplantation for medical reasons. We assume that the remaining patients were evaluated for inclusion in the study rather than evaluated at the end of the study. This is because data presented within the bar charts for the $\mathrm{CHX}$ group indicate substantial losses to follow-up throughout the trial in the $\mathrm{CHX}$ group (44\% by day 33). Losses from the control groups are not reported but include 2 deaths in the control group early on post-transplant

\begin{tabular}{lll}
\hline $\begin{array}{l}\text { Selective reporting (re- } \\
\text { porting bias) }\end{array}$ & Low risk & $\begin{array}{l}\text { All outcomes are reported graphically with bars representing a measure of } \\
\text { variance (assumed to be SD) in the graphs. We estimated the mean scores and } \\
\text { SDs for each outcome so that we could include the data in meta-analyses }\end{array}$ \\
\hline Other bias & Unclear risk & $\begin{array}{l}\text { Quote: "All clinical indexes were scored by the same examiner" } \\
\text { Thent: there is no mention of training for the clinical examiner. } \\
\text { type of transplant, graft-versus-host disease prophylaxis and graft-versus-host } \\
\text { disease. The baseline plaque and gingivitis scores appear balanced between } \\
\text { the groups. The control group appears to have had less extrinsic staining at } \\
\text { baseline but the statistical significance of the difference is not reported }\end{array}$
\end{tabular}

Fine 1985

\section{Study characteristics}

Methods

Trial design: parallel ( 3 arms - 2 arms are relevant to this review) 
Fine 1985 (Continued)

Location: dental practice in the UK

Number of centres: 1

Study duration: the recruitment period is not stated. Study duration and duration of rinsing was 4 months

Participants: quote: "patients coming to the surgery"
Inclusion criteria: quote: "patients had to have some degree of gingival inflammation. A gingival score
system was chosen to measure the degree of inflammation present, and patients selected were re-
quired to have a total score of greater than 36 points"
Exclusion criteria: quote: "No patient under the age of 12 years was included"
Baseline gingivitis: not reported
Age at baseline (years): range not stated but minimum age was 12
Gender: not reported
Number randomised: 133 (Gp A: $45, G p$ B: 50, Gp C: 38$)$
Number evaluated: 116 - breakdown not specified

\section{Interventions Comparison: $(\mathrm{CHX}$ rinse $+\mathrm{OH})$ versus $(\mathrm{PVP} \text { rinse }+\mathrm{OH})^{\star}$ versus $\mathrm{OH}$}

$\mathrm{Gp} \mathrm{A}(\mathrm{n}=45)$ : $\mathrm{CHX}$ (concentration not reported) $+\mathrm{OH}$ (toothbrushing plus floss and/or wood points), volume not reported, once daily, 30 seconds, 4 months

${ }^{\star} \mathrm{Gp} \mathrm{B}(\mathrm{n}=50): \mathrm{PVP}+\mathrm{OH}$ (toothbrushing plus floss and/or wood points), volume not reported, once daily, 30 seconds, 4 months. We excluded this arm from our risk of bias and analysis

Gp C ( $n=38): \mathrm{OH}$ (toothbrushing plus floss and/or wood points), 4 months

Prophylaxis at baseline: quote: "At the first visit thorough scaling and polishing...were given"

OHI: quote: "At the first visit thorough... instruction in oral hygiene techniques were given"

Non-supervised rinsing

Timing of toothbrushing in relation to mouthrinsing: not reported

Duration of treatment: 4 months

Outcomes

Gingivitis ('a gingival inflammation index'). Total score was recorded and presented in results; assessed at 1 week, 1 month, and 4 months follow-up

\begin{tabular}{|c|c|}
\hline Funding & Not reported but Napp Laboratories helped with the statistical analysis \\
\hline \multirow[t]{4}{*}{ Notes } & Sample size calculation: not reported \\
\hline & $\begin{array}{l}\text { Adverse effects: quote: "Several patients having been on chlorhexidine-gluconate mouthwash previ- } \\
\text { ously preferred the PVP-I as it did not stain the teeth or fillings... There was also marked differences } \\
\text { in the individual tendency to develop extrinsic dental staining after using chlorhexidine as a mouth } \\
\text { rinse... Patients also reported a drop in the number of aphthous ulcers whilst using PVP-I" }\end{array}$ \\
\hline & Declarations/conflicts of interest: none stated \\
\hline & $\begin{array}{l}\text { There appears to be an error in Figure } 1 \text {. Outcome data were estimated from the graph at } 3 \text { months but } \\
\text { as the outcome was not measured at } 3 \text { months we assumed that these data related to the outcome at } 4 \\
\text { months }\end{array}$ \\
\hline
\end{tabular}

\section{Risk of bias}


Fine 1985 (Continued)

\section{Bias Authors' judgement Support for judgement}

Random sequence genera- Low risk tion (selection bias)
Quote: "Once the patient had been selected... he/she was then put into one of three groups by completely random allocation according to a random allocation code"

Comment: adequate method of random sequence generation

Allocation concealment High risk
(selection bias)

Quote: "Once the patient had been selected... he/she was then put into one of three groups by completely random allocation according to a random allocation code." This was done by the dental nurse

Comment: the use of an open random allocation schedule means that the practice dental nurse could likely foresee assignment and there is a possibility that randomisation could have been subverted

\begin{tabular}{|c|c|c|}
\hline $\begin{array}{l}\text { Blinding of participants } \\
\text { and personnel (perfor- } \\
\text { mance bias) } \\
\text { All outcomes }\end{array}$ & High risk & $\begin{array}{l}\text { Comment: participant blinding was not possible for } \mathrm{CHX} \text { rinse plus oral hy- } \\
\text { giene versus oral hygiene alone }\end{array}$ \\
\hline $\begin{array}{l}\text { Blinding of outcome as- } \\
\text { sessment (detection bias) } \\
\text { All outcomes }\end{array}$ & High risk & $\begin{array}{l}\text { Quote: "During the visit neither the dentist nor the hygienist knew which group } \\
\text { the patient was in" } \\
\text { Comment: since this study was conducted in a single practice, and randomisa- } \\
\text { tion was done by the dental nurse, it is unlikely that blinding was maintained. } \\
\text { In addition, tooth staining was noted to have occurred in the } \mathrm{CHX} \text { group }\end{array}$ \\
\hline
\end{tabular}

\begin{tabular}{|c|c|c|}
\hline $\begin{array}{l}\text { Incomplete outcome data } \\
\text { (attrition bias) }\end{array}$ & Unclear risk & $\begin{array}{l}\text { Attrition at } 3 \text { months (all study arms): } 17 / 133(12.78 \%) \text {. Losses not broken } \\
\text { down by group. Reasons for losses not reported }\end{array}$ \\
\hline
\end{tabular}

All outcomes down by group. Reasons for losses not reported

\begin{tabular}{|c|c|c|}
\hline \multirow{2}{*}{$\begin{array}{l}\text { Selective reporting (re- } \\
\text { porting bias) }\end{array}$} & \multirow[t]{2}{*}{ High risk } & Outcome measured at 1 and 4 months and reported in graphs \\
\hline & & $\begin{array}{l}\text { Gingivitis: quote: "a gingival inflammation index" reported as 'total score'. No } \\
\text { information on the variance of the total score is provided. The data show an } \\
\text { unusual increase in mean gingivitis score for } \mathrm{CHX} \text { at } 3 \text { months which is not dis- } \\
\text { cussed }\end{array}$ \\
\hline \multirow[t]{2}{*}{ Other bias } & Unclear risk & $\begin{array}{l}\text { There were } 2 \text { examiners: a dentist and hygienist. There is no reference to cali- } \\
\text { bration }\end{array}$ \\
\hline & & $\begin{array}{l}\text { Baseline demographic data are not reported. Mean total gingivitis scores ap- } \\
\text { pear similar in Figure } 1\end{array}$ \\
\hline
\end{tabular}

\section{Study characteristics}

\begin{tabular}{ll} 
Methods & Trial design: parallel (4 arms - 2 arms relevant to this review) \\
Location: USA. Setting not reported & Number of centres: assumed to be 1 \\
Study duration: recruitment period not stated. Study duration and duration of rinsing was 6 months & Participants: quote: "patients with naturally-occurring gingivitis" \\
\hline Participants & $\mathbf{8 6}$ \\
\hline Chlorhexidine mouthrinse as an adjunctive treatment for gingival health (Review) \\
Copyright ( 2017 The Cochrane Collaboration. Published by John Wiley \& Sons, Ltd.
\end{tabular}


Inclusion criteria: quote: "1. Minimum of 16 natural teeth including at least 4 molars, not including third molars. 2. Demonstrated Bleeding on Probing (BOP) using a standard periodontal probe at screening examination and an electronic pressure sensitive probe* $(25 p)$ at baseline examination at a minimum of 6 sites on a minimum of 4 teeth. These sites must have been equal to or less than $4 \mathrm{~mm}$ pocket probing depth and were considered sites with moderate to severe gingivitis"

Exclusion criteria: quote: "1. Systemic disorders or medications which might influence the appearance of the oral tissue or the nature of the plaque. 2. History of adverse reactions which might affect the participation in this study. 3. Used systemic antibiotics for a period of more than 7 days 30 days prior to the baseline examination, or 10 days prior to either the 3 month or 6 month examinations. 4. Diabetics, pregnant or lactating women, or patients on long-term anti-inflammatory medications"

Baseline gingivitis: (GI) Gp A: mean 0.52 (+/- 0.04); Gp B: mean 0.56 (+/- 0.05); Gp C: mean 0.51 (+/- 0.04); Gp D: mean $0.54(+/-0.04)$

Age at baseline (years): mean age 36.6 years. Range not stated

Gender: not reported

Number randomised: 222 (Gp A: 58, Gp B: 55, Gp C: 54, Gp D: 55)

Number evaluated: 175 (Gp A: 43, Gp B: 40, Gp C: 44, Gp D: 48)

Interventions

\section{Comparison: $\mathrm{CHX}$ rinse versus $\mathrm{CHX}$ irrigationa versus water irrigation ${ }^{\mathrm{b}}$ versus toothbrushing}

Gp A ( $n=58)$ : CHX (Peridex) 0.12\%, $15 \mathrm{~mL}$, twice daily, 30 seconds, 6 months

aGp B ( $n=55)$ : CHX irrigation $0.06 \%, 200 \mathrm{~mL}$, once daily, time not reported, 6 months. We excluded this arm from our risk of bias and analysis

bGp C ( $n=54)$ : water irrigation, $500 \mathrm{~mL}$, repeat not reported, time not reported, 6 months. We excluded this arm from our risk of bias and analysis

Gp D ( $n=55)$ : toothbrushing, volume N/A, frequency N/A, time not reported, duration N/A

Prophylaxis at baseline: quote: "Patients received a full mouth professional removal of supra- and subgingival plaque and calculus and a prophylaxis after the baseline and 6-month examinations"

OHI: quote: "During the entire course of the study subjects were encouraged to continue to practice their usual oral hygiene and no additional oral hygiene instructions were given, except that all patients used the same dentifrice"

Non-supervised rinsing

Timing of mouthrinsing in relation to toothbrushing: not reported

Outcomes

Gingivitis (gingival inflammation: GI, Löe 1967; gingival bleeding: BOP yes/no using pressure sensitive probe 25 p); plaque (PI, Silness \& Löe 1964); extrinsic tooth stain (Staining Index, photo of facial surfaces of the 12 anterior maxillary and mandibular teeth were graded for (1) overall stain, (2) stain of each anterior surface, (3) \% coverage against 2 sets of established photo standards); supragingival calculus (Volpe-Manhold Calculus Index, Volpe 1965); PPD (using pressure sensitive probe 25 p) - not relevant to this review; assessed at 3 and 6 months follow-up

Quote: "This study was supported by the Teledyne Research Assistance Program"

Notes

Sample size calculation: not reported

Adverse effects: quotes: "No predominant reasons for not completing the study was found. The most frequent reasons were patient not available for examination, tooth staining, and pregnancy. Adverse soft tissue reactions consisting of epithelial desquamation of the ventral surface of the tongue resulted in the exclusion of one patient from the study who irrigated with chlorhexidine" and "Both CHX irrigation and rinse resulted in significant $(P \leq 0.05)$ increase in the calculus index and stain compared to toothbrushing and water irrigation" 
Flemmig 1990 (Continued)

Declarations/conflicts of interest: none stated

\section{Risk of bias}

\section{Bias}

Random sequence genera- Unclear risk tion (selection bias)

\section{Authors' judgement Support for judgement}

Quote (from report): "After the baseline examination patients were assigned to one of the following treatment groups after balancing according to sex and marginal gingival bleeding"

Quote (from correspondence): "Treatment was assigned randomly... the study protocol is not available"

Comment: no description of the method of random sequence generation

$\begin{array}{ll}\begin{array}{l}\text { Allocation concealment } \\ \text { (selection bias) }\end{array} & \begin{array}{l}\text { Quote (from correspondence): "..allocation was concealed... the study proto- } \\ \text { col is not available" }\end{array} \\ & \text { Comment: not enough information is provided }\end{array}$

Blinding of participants High risk and personnel (performance bias)

All outcomes
The authors report that this is a single-blind study which must apply to the examiner, since participants could not be blinded (irrigation versus rinse versus toothbrushing alone)

Quote: "At 6 months calculus was increased by $273.2 \%$ in the $\mathrm{CHX}$ rinse.. group compared to the toothbrushing group. In the $\mathrm{CHX}$ rinsing group stain at the facial surfaces of anterior teeth was increased by $74.2 \%$... compared to toothbrushing"

Comment: the higher level of tooth staining in the $\mathrm{CHX}$ group meant that participants could have worked out which group they were in and this could have affected their oral health behaviours and hence the outcome. The direction of this potential bias is unclear

\section{Blinding of outcome as- High risk} sessment (detection bias) All outcomes
The authors report that this is a single-blind study which must apply to the examiner, since participants could not be blinded (irrigation versus rinse versus toothbrushing alone). Examiner 1 assessed soft tissues and the plaque index. The other examiners each assessed 1 of the remaining parameters (GI, pocket probing depth and BOP and Calculus Index). Each clinical parameter was assessed by the same examiner throughout the study. Tooth discolouration and staining was assessed in a single-blind manner. Quote: "At 6 months calculus was increased by $273.2 \%$ in the $\mathrm{CHX}$ rinse.. group compared to the toothbrushing group. In the $\mathrm{CHX}$ rinsing group stain at the facial surfaces of anterior teeth was increased by $74.2 \%$... compared to toothbrushing"

Comment: the higher level of staining in the $\mathrm{CHX}$ group meant that the outcome assessor could not be adequately blinded and this could have affected the outcome assessment. The direction of this potential bias is unclear

Incomplete outcome data High risk
(attrition bias)
All outcomes

Attrition 22/113 (19.5\%). Attrition by group: CHX 15/58 (25.86\%), toothbrushing (normal oral hygiene) 7/55 (12.73\%). Losses higher in CHX group. Reasons for loss to follow-up (not broken down by group): patient not available for examination, tooth staining and pregnancy. Insufficient information to determine if losses are due to the intervention but authors report a significant increase in stain in the rinse group compared to the toothbrushing group at 6 months and staining was one of the reasons for loss to follow-up

All outcomes stated in the methods section are adequately reported in the results section. Bacteriological results are presented in another paper

Selective reporting (re- Low risk
porting bias)



analyses

\begin{tabular}{ll}
\hline Other bias & $\begin{array}{l}\text { There were } 4 \text { examiners, each measuring a different outcome. No measure of } \\
\text { intraexaminer reliability for each index is given }\end{array}$ \\
$\begin{array}{l}\text { Comment: there is no mention of training or calibration of the clinical examin- } \\
\text { ers }\end{array}$ \\
Baseline demographic data by group are not reported
\end{tabular}

\section{Flotra 1972}

\section{Study characteristics}

Trial design: parallel (4 arms). *Note: data are reported for the 3 treatment groups combined. There-
fore, there are 2 overall groups in the analysis: control and experimental
Location: examinations were conducted at the military garrison at Rygge, approximately 50 kilometres
south of Oslo, Norway
Number of centres: 1
Study duration: recruitment period not stated. Study duration and duration of rinsing was 17 weeks
Participants: Norweigan Air Force soldiers
Inclusion criteria: not reported
Exclusion criteria: not reported
Baseline gingivitis: (GI): Gp A: mean 1.11; Gp B: 1.22
Age at baseline (years): mean age: $27 ;$ range: $19-39$
Gender: not reported
Number randomised: 50 (Gp A: $40, \mathrm{Gp} B: 10)$
Number evaluated: 28 (Gp A: $22, \mathrm{Gp} B: 6)$

\section{Interventions \\ Comparison: $0.2 \% \mathrm{CHX}$ gluconate versus $0.1 \% \mathrm{CHX}$ gluconate versus $0.1 \% \mathrm{CHX}$ acetate versus benzene alcohol control}

Gp A $(n=40): 0.2 \% \mathrm{CHX}$ rinse, $0.1 \% \mathrm{CHX}$ rinse, $0.1 \% \mathrm{CHX}$ acetate rinse, $10 \mathrm{~mL}$, twice daily, $1 \mathrm{~min}, 17$ weeks

Gp B ( $n=10)$ : control (benzene alcohol),10 mL, twice daily, 1 min, 17 weeks

Prophylaxis at baseline: none at baseline. Supra- and subgingival scaling was performed during the study starting during week 9

OHI: none given. Quote: "The established oral hygiene routine was not otherwise changed"

Non-supervised mouthrinsing

No information provided on timing of rinsing in relation to toothbrushing or postrinse instructions

Outcomes

Gingivitis (gingival inflammation: GI Löe \& Silness 1963); plaque (PI Silness \& Löe 1964); calculus (Retention Index, Bjorby \& Löe 1966); and pocket depth (mm) - not relevant for this review; assessed at 8, 11, and 17 weeks follow-up 
Flotra 1972 (Continued)

Funding
$\begin{aligned} & \text { Not reported } \\ & \text { Sample size calculation: not reported } \\ & \text { Adverse effects: quote: "The taste of the control solution, like the test solution, was some-what un- } \\ & \text { pleasant" } \\ & \text { Side effects are reported in a separate publication. During the experimental period, } 11 \text { participants } \\ & \text { exhibited lesions in the oral mucosa ( } 10 \mathrm{CHX} \text { and } 1 \text { control). } 3 \text { of the } 10 \text { participants developed more } \\ & \text { severe lesions ( } 2 \text { in the } 0.2 \% \mathrm{CHX} \text { gluconate group and } 1 \text { in the } 0.1 \% \mathrm{CHX} \text { acetate group). } 1 \text { individual } \\ & \text { (0.1\% CHX gluconate) developed what appeared to be a viral stomatitis and another ( } 0.1 \% \mathrm{CHX} \text { ac- } \\ & \text { etate) a viral parotitis. } 1 \text { individual developed small ulcers under the upper lip - probably due to an } \\ & \text { aphthous lesion. In the test groups at } 4 \text { weeks ( } \mathrm{n}=48), 12 \% \text { of the tooth surfaces without fillings and } \\ & 62 \% \text { of the silicate fillings became discoloured while } 36 \% \text { of the test persons developed discoloured } \\ & \text { tongues during the experimental period } \\ & \text { Declarations/conflicts of interest: none stated }\end{aligned}$

\section{Risk of bias}

Bias Authors' judgement Support for judgement

Random sequence genera- Low risk tion (selection bias)
Randomisation is not mentioned in the study report

Quote (from correspondence): "The allocation of participants to the various groups was performed in this way: First we decided how many we wanted in each group, the appropriate no of lots for each group were made and drawn for each participant from a jar"

Comment: adequate method of random sequence generation

Allocation concealment Low risk
(selection bias)

(selection bias)

\section{Allocation concealment is not mentioned in the study report}

Quote (from correspondence): "The drawing of lots was performed by Dr Waerhaug and myself (Prof Gjermo), with particular attention that the group allocation was not revealed for Dr Flötra who performed the examinations. This was done at the Department of Periodontology at the University of Oslo, whereas the actual examinations were performed at a military garrison at Rygge, approximately $50 \mathrm{~km}$ south of Oslo. Dr Flötra brought the results to Oslo before the seal for the allocation was broken and data analyzed"

Comment: we consider it unlikely that participants or investigators enrolling participants could foresee assignments
Blinding of participants High risk and personnel (performance bias)

All outcomes
Quote: "The experiment was a double-blind study." Additional information was obtained from the second author of the paper: "neither the examiners nor the participants were aware of which group they belonged to." The control mouthrinse contained $0.5 \%$ benzene alcohol

Quotes: "The test mouth washes were distributed in coded plastic bottles.." and "The taste of the control solution, like the test solution was somewhat unpleasant"

In the test groups, $12 \%$ of the tooth surfaces and $62 \%$ of the silicate fillings became discoloured while $36 \%$ of the test persons developed discoloured tongues during the experimental period

Comment: the higher level of tooth staining in the $\mathrm{CHX}$ group meant that participants could have worked out which group they were in and this could have affected their oral health behaviours and hence the outcome. The direction of this potential bias is unclear 
Flotra 1972 (Continued)

Blinding of outcome as- High risk sessment (detection bias)

All outcomes

Quote: "The experiment was a double-blind study." Additional information was obtained from the second author of the paper: "neither the examiners nor the participants were aware of which group they belonged to" and "One investigator recorded the Plaque Index throughout the study and another recorded the gingival index" and "Dr Flötra brought the results to Oslo before the seal for the allocation was broken and data analyzed"

In the test groups, $12 \%$ of the tooth surfaces and $62 \%$ of the silicate fillings became discoloured while $36 \%$ of the test persons developed discoloured tongues during the experimental period

Comment: despite the efforts taken to maintain blinding, the higher level of staining in the $\mathrm{CHX}$ group meant that the outcome assessor could not be adequately blinded and this could have affected the outcome assessment. The direction of this potential bias is unclear

Incomplete outcome data High risk (attrition bias)

From Table 1 and $2 \mathrm{n}=28$ at 17 weeks. 22/50 (44\%) lost to follow-up at 17 weeks. By group: Gp A 18/40 (45\%); Gp B 4/10 (40\%). Reasons for losses given for 11/22: 2 excluded because of stomatitis and parotitis, 9 for various reasons such as discharge from service, transfers, etc

Comment: losses to follow-up although proportionately balanced between the groups were unacceptably high

Selective reporting (re- High risk
porting bias)

The outcome data for the $3 \mathrm{CHX}$ rinse groups are not reported by individual rinse group; combined data for the $3 \mathrm{CHX}$ groups are presented in the results. The results for the Retention Index are not reported fully. Pocket probing depth is measured at baseline but not reported. Side effects including oral lesions, discolouration of the teeth and tongue are reported in detail in a separate publication although not in a format that would allow their inclusion in meta- analyses

\begin{tabular}{ll}
\hline Other bias & Unclear risk \\
& $\begin{array}{l}\text { Baseline demographic data are not reported. Mean } \mathrm{GI} \text { and } \mathrm{PI} \text { in the } 2 \text { groups at } \\
\text { baseline appear similar (not significantly different for } \mathrm{GI} \text { ) }\end{array}$
\end{tabular}

Graziani 2015

\section{Study characteristics}

\begin{tabular}{ll}
\hline Methods & Trial design: parallel (4 arms) \\
Location: Unit of Dentistry and Oral Surgery, University Hospital of Pisa, Italy \\
Number of centres: 1 \\
Study duration: the recruitment period is not stated. Study duration and duration of rinsing was 5 \\
weeks \\
Participants: healthy adults \\
Inclusion criteria: at least 20 teeth, the 8 incisors free of restorations or prosthetic reconstructions, gen- \\
eral good health \\
$\begin{array}{l}\text { Exclusion criteria: systemic illness (including cardiovascular, renal of liver diseases), smokers, peri- } \\
\text { odontal/dental treatment within the } 35 \text { days preceding the study, pregnant or lactating females, un- } \\
\text { dergoing orthodontic treatment, inability to sign consent form }\end{array}$
\end{tabular}


Baseline gingivitis: (Löe \& Silness GI) Gp A: mean 1.8 (95\% Cl 1.4 to 2.2); Gp B: mean 1.6 (95\% Cl 1.3 to 1.9); Gp C: mean 1.8 (95\% Cl 1.5 to 2.2$)$; Gp D: mean 1.5 (95\% $\mathrm{Cl} 1.3$ to 1.8 )

Age at baseline (years): Gp A: mean 29.7 (95\% Cl 23.4 to 35.9); Gp B: mean 39.5 (95\% Cl 31.6 to 35.9); Gp C: mean 36 (95\% Cl 30.3 to 37.2); Gp D: mean 29.7 (95\% Cl 24 to 35.5)

Gender: overall: male 36 (51\%), female 34 (49\%). Gp A: male 6 (40\%), female 9 (60\%); Gp B: male 11 (52\%), female 10 (48\%); Gp C: male $10(53 \%)$, female $9(47 \%)$; Gp D: male $9(60 \%)$, female $6(40 \%)$

Number randomised: 70 (Gp A: 15; Gp B: 21; Gp C: 19; Gp D: 15)

Number evaluated: 70 (Gp A: 15; Gp B: 21; Gp C: 19; Gp D: 15)

Interventions

\section{Comparison: $\mathrm{CHX}$ rinse 1 versus $\mathrm{CHX}$ rinse 2 versus $\mathrm{CHX}$ rinse 3 versus control rinse}

Gp A ( $n=15):$ CHX 0.2\% (with alcohol), $10 \mathrm{~mL}$, twice daily, 1 min, 5 weeks

Gp B ( $n=21):$ CHX 0.2\% (alcohol-free), $10 \mathrm{~mL}$, twice daily, 1 min, 5 weeks

Gp C ( $n=19)$ : CHX 0.2\% with antidiscolouration system (alcohol-free), $10 \mathrm{~mL}$, twice daily, $1 \mathrm{~min}, 5$ weeks

Gp D ( $n=15): \mathrm{H}_{2} \mathrm{O} / \mathrm{NaCl}$ control, $10 \mathrm{~mL}$, twice daily, 1 min, 5 weeks

Prophylaxis at baseline: supragingival scaling and polishing

OHI: quote: "Oral hygiene instructions were provided... as follows: interdental brushing... and/or dental floss when interdental embrasures did not allow interdental brushing. Interdental cleaning was followed by electric toothbrushing... A sodium lauryl sulfate-free toothpaste was provided to each participant"

Non-supervised rinsing

Timing of mouthrinsing in relation to toothbrushing: not reported

Postrinsing instructions: participants were instructed to refrain from tea, coffee and red wine consumption for at least 1 hour after rinsing

Outcomes

Gingivitis (gingival inflammation: GI Löe \& Silness 1963; gingival bleeding: full-mouth bleeding score, Ainamo and Bay 1975); plaque (full-mouth plaque score, O'Leary 1972); tooth discolouration/staining (staining index, modification of Lobene 1968, Grundemann 2000) using digital photographic measurements of the buccal surfaces of the 8 incisors, tooth colour was measured using spectrophotometric analysis; other adverse effects; assessed at 1,3 and 5 weeks follow-up

Funding

Quote: "Johnson \& Johnson supported this study with a grant to purchase the Vita EasyShade spectrophotometer and to perform the data analysis (Dr D'Aiuto). UCLH/UCL received a proportion of funding from the Department of Health's NIHR Biomedical Research Centres funding scheme"

Notes

Sample size calculation: sample size was geared towards comparing each treatment mean with the control mean. The calculation was reported and achieved. Quote: "A sample size of 12 individuals per group, with at least three subjects to compensate for dropouts, with 3 treatment arms and one control group would provide any-pair power of $80 \%(\alpha=0.05)$ (Kruskal-Wallis Test) for comparing each treatment mean with the control mean"

Adverse effects: "At 35 days, staining was frequent, reported in well above $60 \%$ of all cases rinsing with the three CHX formulations." Tongue staining incidence: Gp A: 1 at 35 days; Gp B: 2 at 21 days; Gp C: 2 at 7 and 21 days. Taste alteration: Gp A: 2 at 7 days; Gp B: 2 at 7 days and 2 at 21 days; Gp C: 1 at 35 days. Gastric acidity: Gp B: 1 at 7 days. No serious side effects reported

Non-smokers

Declarations/conflicts of interest: quote: "Dr Graziani has received lecture fees from Johnson \& Johnson and from Curaden healthcare" (Johnson \& Johnson and Curaden were the manufacturers of 2 of the $3 \mathrm{CHX}$ rinses) 
Graziani 2015 (Continued)

Participants in the study were described as having "mild periodontal involvement and periodontal in-

flammation limited to approximately one-fifth of the entire dentition"

We combined groups A, B and C for the main analysis

\section{Risk of bias}

\section{Bias Authors' judgement Support for judgement}

Random sequence genera- Low risk tion (selection bias)
Quote: "a computer-generated random sequence was used to assign participants to one of the four treatment groups"

Comment: adequate method of random sequence generation
Quote: "random sequence conducted by a research fellow not directly involved in the experiments was used to assign participants... Allocation to the treatment was concealed as a code number identifying the allocated group. It was sealed in an opaque envelope which was opened at the baseline visit after completion of clinical measurements, photographs, supragingival scaling and polishing"

Comment: numerous steps were taken to ensure that the random sequence was applied exactly as it was generated without any manipulation

Blinding of participants High risk
and personnel (performance bias)

All outcomes

Quotes: "double-blind" and "the mouthrinse bottles were dispensed to the participants according to the allocated group by a research fellow not directly involved in the research. The control rinse was saline"
Quote: "At 35 days, staining was frequent, reported in well above $60 \%$ of all cases rinsing with the three $\mathrm{CHX}$ formulations"

Comment: the higher level of tooth staining in the $\mathrm{CHX}$ groups meant that participants could have worked out which group they were in (or that they were in 1 of the active mouthrinse groups) and this could have affected their oral health behaviours and hence the outcome. The direction of this potential bias is unclear

\begin{tabular}{ll}
\hline Blinding of outcome as- & High risk \\
sessment (detection bias) &
\end{tabular}
All outcomes

Quotes: "double-blind" and "Clinical examinations were performed... by an examiner unaware of the group allocation"

Comment: the higher level of staining in the $\mathrm{CHX}$ groups meant that the outcome assessor could not be adequately blinded and this could have affected the outcome assessment. The direction of this potential bias is unclear

\begin{tabular}{|c|c|c|}
\hline $\begin{array}{l}\text { Incomplete outcome data } \\
\text { (attrition bias) } \\
\text { All outcomes }\end{array}$ & Low risk & $\begin{array}{l}\text { All randomised participants were included in the analyses (ITT analysis) } \\
\text { Attrition at } 35 \text { days was } 17 / 70 \text { (24.3\%). By group: Gp A 4/15 (26.7\%); Gp B 3/21 } \\
\text { (14.3\%); Gp C 4/19 (21.1\%); Gp D 6/15 (40\%) }\end{array}$ \\
\hline
\end{tabular}

\begin{tabular}{|c|c|c|}
\hline $\begin{array}{l}\text { Selective reporting (re- } \\
\text { porting bias) }\end{array}$ & High risk & $\begin{array}{l}\text { Percentage reduction in full-mouth bleeding score was not reported for Gp } \\
\text { A. Due to incomplete reporting of this outcome, data on full-mouth bleeding } \\
\text { score could not be included in a meta-analysis }\end{array}$ \\
\hline Other bias & High risk & $\begin{array}{l}\text { Full-mouth bleeding score measurement was carried out by a "blinded, cali- } \\
\text { brated and experienced examiner." Apart from that statement, training and } \\
\text { calibration of the } 1 \text { outcome assessor are not mentioned. The } 4 \text { groups do not } \\
\text { appear to be balanced with respect to mean age, } \% \text { periodontitis diagnosed, } \\
\text { mean number of pockets and } \% \text { of pockets } \geq 5 \mathrm{~mm} \text {. The } 95 \% \text { confidence inter- } \\
\text { vals indicate some statistically significant differences between the groups but } \\
\text { the P values for the differences are not reported }\end{array}$ \\
\hline
\end{tabular}




\section{Study characteristics}

\begin{tabular}{|c|c|}
\hline \multirow[t]{4}{*}{ Methods } & Trial design: parallel ( 2 arms) \\
\hline & Location: USA. Quote: "Dental office conditions" \\
\hline & Number of centres: assumed to be 1 \\
\hline & Study duration: recruitment period not stated. Study duration and duration of rinsing was 6 months \\
\hline \multirow[t]{8}{*}{ Participants } & Participants: adults \\
\hline & $\begin{array}{l}\text { Inclusion criteria: quote: "To be accepted into the study, subjects had to have at least } 16 \text { natural teeth, } \\
\text { including } 4 \text { molars" }\end{array}$ \\
\hline & $\begin{array}{l}\text { Exclusion criteria: quote: "Subjects were not enrolled if they had existing soft-tissue damage due to ill- } \\
\text { fitting appliances, evidence of overt periodontal disease or gross dental neglect, an unfavorable med- } \\
\text { ical history, or a history of routine antibiotic use" }\end{array}$ \\
\hline & $\begin{array}{l}\text { Baseline gingivitis: (GI) examiner A (Gp A: mean 0.5530, Gp B: mean 0.5388); examiner B (Gp A: mean } \\
0.7472 \text {, Gp B: mean 0.7470) }\end{array}$ \\
\hline & Age at baseline (years): Gp A: 33.02; Gp B: 32.25. Range Gp A: 18-58; Gp B: 18-60 \\
\hline & Gender: Gp A: male 72 (33\%), female 147 (67\%); Gp B: male 71 (34\%), female 140 (66\%) \\
\hline & Number randomised: 430 (Gp A: 219, Gp B: 211) \\
\hline & Number evaluated: 380 (Gp A: 187, Gp B: 193) \\
\hline
\end{tabular}

\begin{tabular}{|c|c|}
\hline \multirow[t]{8}{*}{ Interventions } & Comparison: CHX versus placebo \\
\hline & Gp A ( $n=187):$ CHX 0.12\%,15 mL, twice daily, 30 seconds, 6 months \\
\hline & Gp B ( $n=193)$ : placebo, $15 \mathrm{~mL}$, twice daily, 30 seconds, 6 months \\
\hline & $\begin{array}{l}\text { Prophylaxis at baseline: quote: "All subjects received a thorough prophylaxis after the baseline exami- } \\
\text { nation for plaque, gingivitis, and oral soft tissue health" }\end{array}$ \\
\hline & $\begin{array}{l}\text { OHI: quote: "A sodium fluoride dentifrice (Crest) and soft toothbrushes were given to each subject to be } \\
\text { used according to their own individual habits" }\end{array}$ \\
\hline & Non-supervised mouthrinsing \\
\hline & No information is provided on timing of rinsing in relation to toothbrushing or postrinse instructions \\
\hline & Duration of treatment: 6 months \\
\hline Outcomes & $\begin{array}{l}\text { Gingivitis (gingival inflammation (GI, Löe 1967); gingival bleeding (GI sites with scores } 2 \text { or } 3 \text { indicating } \\
\text { bleeding)); plaque (TQH, Turesky 1970); oral soft tissue effects; calculus (index not reported); and tooth } \\
\text { stain (index not reported) and tongue stain assessed at } 3 \text { and } 6 \text { months follow-up }\end{array}$ \\
\hline Funding & $\begin{array}{l}\text { Quote: "The support of this study by the Procter \& Gamble Company, Cincinnati, Ohio, is gratefully ac- } \\
\text { knowledged" }\end{array}$ \\
\hline \multirow[t]{2}{*}{ Notes } & Sample size calculation: not reported \\
\hline & $\begin{array}{l}\text { Adverse effects: quotes: "As expected with the use of any oral antimicrobial, some extrinsic tooth stain- } \\
\text { ing was observed in the chlorhexidine group" and "Supragingival calculus was higher in the group us- } \\
\text { ing chlorhexidine" and "The systematic evaluation of the oral structures did not yield any indication of } \\
\text { chlorhexidine-related changes in the oral mucosa" }\end{array}$ \\
\hline
\end{tabular}


Grossman 1986 (Continued)

Declarations/conflicts of interest: none stated

Gingivitis data were extracted for examiner A only

\section{Risk of bias}

Bias Authors' judgement Support for judgement

Random sequence genera- Unclear risk

tion (selection bias)

Quote: "subjects were stratified by sex, age and gingivitis severity. Within strata they were assigned to one of two treatment groups by random permutations of $2 "$

Comment: no description of the method of random sequence generation

Allocation concealment $\quad$ Unclear risk $\quad$ Comment: no information is provided
(selection bias)

(selection bias)

\section{Blinding of participants High risk} and personnel (perfor-

mance bias)

All outcomes
Quotes: ".. double-blind clinical trial" and "Neither the subject nor the dental examiners knew which treatment the subject was assigned to at any time during the study." The control mouthrinse was identical to the $\mathrm{CHX}$ mouthrinse but without the $\mathrm{CHX}$ gluconate

Quote: "more extrinsic tooth staining was observed in the chlorhexidine group"

Comment: the higher level of tooth staining in the active group meant that participants could have worked out which group they were in and this could have affected their oral health behaviours and hence the outcome. The direction of this potential bias is not clear

\section{Blinding of outcome as- Low risk} All outcomes sessment (detection bias)

Quotes: ".. double-blind clinical trial" and "Neither the subject nor the dental examiners knew which treatment the subject was assigned to at any time during the study" and "gingivitis was measured independently by two examiners" and "Prior to the 6-month examination, a subset of subjects was fitted with custom-made tooth covers fabricated from opaque plastic. This procedure was included to eliminate bias due to chlorhexidine related tooth stain. The subjects' tongues were also coated with a paste mixture of food colors to eliminate the chance of bias which could result from stained tongues during the gingivitis examinations" and "The GI and bleeding scores recorded by each examiner were consistent regardless of whether subjects were graded with or without tooth covers"

Comment: the presence of tooth staining in the $\mathrm{CHX}$ group did not appear to have an effect on the measurement of gingivitis. The effect of the tooth staining in the $\mathrm{CHX}$ group on plaque is less clear and the measurement of plaque could have been affected if the examiners noticed the increased tooth staining in the active group. It is also unclear how the tooth staining may have influenced recording of outcomes at 3 months when no tooth covers were used

\section{Incomplete outcome data High risk} (attrition bias)

Attrition at 6 months was 50/430 (11.6\%). Attrition by group: $\mathrm{CHX} 32 / 219$

All outcomes people who had moved or could not meet examination schedules"

Comment: assuming the missing participants in 1 group had a higher mean (e.g. gingivitis score) than those in the other group, as the attrition rate increased, so would the mean difference between groups

Selective reporting (re- High risk
porting bias)

Outcome data for gingivitis, occurrence, severity and bleeding are reported separately for examiner A and B. We extracted gingivitis data for examiner A only. Information on the variance of the mean scores for gingivitis and plaque were not reported. We used the SDs for each clinical parameter at baseline so 


\title{
Study characteristics
}

Methods Trial design: parallel (4 arms -2 arms relevant to this review)

Location: assumed to be Northfield, New Jersey, USA. Quote: "Dental office conditions"

Number of centres: 1

Study duration: recruitment period is not stated. Study duration and duration of rinsing was 6 months

Participants

Participants: quote: "adult males and females from the Northfield, New Jersey area"

Inclusion criteria: quote: "To be accepted into the study, subjects had to sign an informed consent, have at least 16 natural teeth (including four molars), and have at least one gingival site that bled on probing"

Exclusion criteria: quote: "Subjects were not enrolled if they had evidence of overt periodontal disease or gross dental neglect, an unfavorable medical history, or a history of routine antibiotic use"

Baseline gingivitis: (GI) Gp A: mean 0.5332, Gp B: mean 0.5227, Gp C: mean 0.5457, Gp D: mean 0.4994

Age at baseline (years): Gp A: mean 36.8, Gp B: mean 37.1, Gp C: mean 35.9, Gp D: mean 36.5. Range not reported

Gender: overall: male 166 (33\%), female 336 (67\%). Gp A: male 35 (31\%), female 78 (69\%); Gp B: male 46 (36\%), female $83(64 \%)$; Gp C: male 42 (33\%), female 85 (67\%); Gp D: male $43(32 \%)$, female $90(68 \%)$

Number randomised: 502 (Gp A: 113, Gp B: 129, Gp C: 127, Gp D: 133). *Note: unclear if this is the number initially randomised

Number evaluated: 481

Note: $\mathrm{n}$ by group taken from Table 1 showing initial balance for subjects completing the study

Interventions

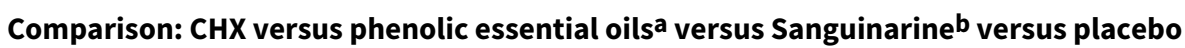 \\ Gp A ( $n=113):$ CHX 0.12\%, $15 \mathrm{~mL}$, twice daily, 30 seconds, 6 months \\ aGp B ( $n=129)$ : phenolic essential oils, $20 \mathrm{~mL}$, twice daily, 30 seconds, 6 months. We excluded this arm \\ from our risk of bias and analysis \\ bGp C ( $n=127)$ : Sanguinarine, $15 \mathrm{~mL}$, twice daily, 15 seconds ( 2 consecutive rinses of 15 seconds twice \\ a day), 6 months. We excluded this arm from our risk of bias and analysis \\ Gp D ( $\mathrm{n}=133)$ : placebo, $15 \mathrm{~mL}$, twice daily, 30 seconds, 6 months \\ Prophylaxis at baseline: quote: "Following baseline examinations... all subjects received a thorough \\ prophylaxis"
}


Grossman 1989 (Continued)

OHI: none given. Quote: "A sodium fluoride dentifrice (Crest, Proctor and Gamble) and soft toothbrushes were given to all subjects to be used according to individual habits"

Non-supervised rinsing

Timing of mouthrinsing in relation to toothbrushing: not reported

Outcomes

Gingivitis (gingival inflammation: GI Löe \& Silness 1967; gingival bleeding: GI grades 2 or 3); plaque (PI, TQH, Turesky 1970); extrinsic tooth stain (photographs of the facial surfaces of the 12 anterior teeth in the maxilla and mandible were graded for dental stain intensity ( $0-4$ scale none-very dark) and coverage (0-6 scale none-heavy)); oral soft tissue health assessed at 3 and 6 months follow-up

$\begin{array}{ll}\text { Funding } & \begin{array}{l}\text { Quote: "The support of this study by the Procter \& Gamble Company, Cincinnati, Ohio is gratefully ac- } \\ \text { knowledged" }\end{array}\end{array}$
knowledged"

Notes

Sample size calculation: not reported

Adverse effects: quote: "As would be expected, the extended use of chlorhexidine resulted in more extrinsic dental stain than in other groups. In contrast to previously published reports using different methods of evaluation, use of the phenolic rinse produced a significant increase in dental stain as well. After 3 and 6 months, those subjects using either chlorhexidine or the rinse containing phenolic compounds experienced a significant increase in stain when compared to those on placebo or the sanguinarine rinse"

Declarations/conflicts of interest: none reported, however some of the authors worked for the Procter \& Gamble Company

\section{Risk of bias}

\begin{tabular}{lll}
\hline Bias & Authors' judgement & Support for judgement \\
\hline $\begin{array}{l}\text { Random sequence genera- } \\
\text { tion (selection bias) }\end{array}$ & Unclear risk & $\begin{array}{l}\text { Subjects were stratified by age and GI score at baseline. Quote: "Within strata, } \\
\text { subjects were randomly assigned to one of four treatments" }\end{array}$ \\
& & Comment: no description of the method of random sequence generation \\
\hline
\end{tabular}

\begin{tabular}{lll}
\hline $\begin{array}{l}\text { Allocation concealment } \\
\text { (selection bias) }\end{array}$ & Unclear risk & No information is provided
\end{tabular}

Blinding of participants and personnel (performance bias)

All outcomes
High risk

The authors state that the study is double-blind. Quotes: "Neither the subjects nor the examiners knew which treatments were assigned at any time during the study" and "All products were supplied to the subjects in identical amber pint bottles." The placebo rinse was Peridex without the $\mathrm{CHX}$

Comment: there was a significantly higher level of staining in the $\mathrm{CHX}$ group compared to the placebo group and this meant that participants could have worked out which group they were in, and this could have affected their oral health behaviours, and hence the outcome. The direction of this potential bias is not clear
Blinding of outcome as- Low risk sessment (detection bias)

All outcomes
At the 6-month examination each subject was graded twice, first without a custom-made plastic tooth cover in place and then with the tooth cover in place. Quote: "In addition the dorsum of the tongue was stained brown with food color to imitate the antimicrobial tongue stain." Analysis of gingivitis scores recorded with and without masking devices revealed that the reductions for each treatment group were comparable

Comment: the presence of tooth staining in the $\mathrm{CHX}$ group did not appear to have an effect on the measurement of gingivitis (our primary outcome). The effect of the tooth staining in the $\mathrm{CHX}$ group on plaque is less clear and the measurement of plaque could have been affected if the examiners noticed the 
increased tooth staining in the active group. It is also unclear how the tooth staining may have influenced recording of outcomes at 3 months when no tooth covers were used

\begin{tabular}{|c|c|c|}
\hline $\begin{array}{l}\text { Incomplete outcome data } \\
\text { (attrition bias) } \\
\text { All outcomes }\end{array}$ & Unclear risk & $\begin{array}{l}\text { Total number randomised is not specified, only number completing. Base- } \\
\text { line data on completers is provided in Table } 1 \text {, but number does not tally with } \\
\text { number of completers reported in the abstract. No details are provided on } \\
\text { number of losses to follow-up }\end{array}$ \\
\hline
\end{tabular}

\begin{tabular}{ll}
\hline $\begin{array}{l}\text { Selective reporting (re- } \\
\text { porting bias) }\end{array}$ & The covariance adjusted mean scores for gingivitis, bleeding and plaque are \\
& presented with the covariance analysis mean squared error term for the 3 and \\
6-month exams. SD was calculated as the sqrt of the covariance analysis mean & squared error so that these data could be included in meta-analysis. Data on \\
mean extrinsic tooth stain accumulation is reported without any information \\
on variance of the mean scores
\end{tabular}

Other bias Unclear risk No information is provided on intra- and interexaminer reproducibility

Quote: "All groups were well-balanced at the beginning of the study with respect to age, gender, initial plaque, stain, gingivitis or bleeding grades"

\section{Study characteristics}

Methods Trial design: parallel, (3 arms - 2 are relevant to this review)

Location: not explicitly stated and authors from Sweden and Finland (setting not reported)

Number of centres: 1

Study duration: recruitment period is not stated. Study duration and duration of rinsing was 4 weeks

Participants Participants: adult males with gingivitis

Inclusion criteria: males aged 18 to 60 years; at least $25 \%$ of all gingival sites ( 6 sites per tooth) having a minimum Löe \& Silness GI score of 2; minimum 10 teeth per jaw; no pocket probing depth greater than $5 \mathrm{~mm}$

Exclusion criteria: people with buccal or extensive interproximal restorations reaching beyond the axial angle of the teeth assigned for the gingival fluid flow measurement; people with crowns or bridgework; previous hypersensitivity or reactions to drugs; alcohol or drug addiction; severe liver or renal disease; psychiatric disorders; severe disability with multiple drug therapy; current antibiotics or antiphlogistics (including sprays with corticosteroids) or anticholinergic antiparkinson or antidepressant treatments

Baseline gingivitis: (\% sites BOP) Gp A: mean 16 (SE unclear); Gp B: mean 14 (SE unclear); Gp C: mean 19 (SE unclear)

Age at baseline (years): Gp A: mean 41 (SE 2); Gp B: mean 41 (SE 2); Gp C: mean 39 (SE 2). Age range 18-60

Gender: males only

Number randomised: 62 (Gp A: 21; Gp B: 20; Gp C: 21)

Number evaluated: 57 (Gp A: 21; Gp B: 18; Gp C: 18)

Interventions

\section{Comparison: $\mathrm{CHX}$ digluconate rinse versus delmopinol hydrochloride rinse* versus placebo rinse}

$\mathrm{Gp} \mathrm{A}(\mathrm{n}=21)$ : $\mathrm{CHX}(0.2 \%), 10 \mathrm{~mL}, 1 \mathrm{~min}$, twice daily (morning and evening) 
Hase 1995 (Continued)

${ }^{*} G p B(n=20)$ : delmopinol hydrochloride $(0.2 \%)$, same routine. We excluded this arm from our risk of bias and analysis

$\mathrm{Gp} \mathrm{C} \mathrm{(}=21)$ : same routine using placebo (flavoured in order to imitate the sensation of rinsing with the active solutions)

Prophylaxis at baseline: supra- and subgingival scaling

$\mathrm{OHI}$ : participants were instructed to brush their teeth without toothpaste

Non-supervised rinsing

Timing of rinsing in relation to toothbrushing: after brushing without toothpaste

Postrinsing instructions: not reported

Duration of treatment: 4 weeks

Outcomes

Quote: "The split-mouth technique was used in the study in order to avoid interference between various assessments." Plaque measurements were made on the left halves of the jaws and gingivitis measurements on the right

Gingivitis (gingival BOP, no reference), measured at 2 and 4 weeks; plaque (PI, Silness \& Löe 1964; plaque wet weight, Scheinin \& Makinen 1971 - not relevant to this review) measured at 4 weeks

Subjective opinion of participants regarding taste, staining (teeth or tongue or both), clean mouth, vesicles or ulcerations or both, burning sensation, transient anaesthetic sensation, all measured at 4 weeks on a 1 to $100 \mathrm{~mm}$ VAS

Gingival fluid flow (Löe \& Holm Pedersen 1965) measured at 2 and 4 weeks

Safety (physical examinations including blood tests and ECG), measured before starting treatment and within 3 weeks after the termination of the study - not relevant to this review

Funding Not reported

Notes

Sample size calculation: not reported. Quote: ".. the number of patients in this study turned out to be too small to yield conclusive results on the effect of either delmopinol or chlorhexidine on pre-established gingivitis"

Adverse effects: results from the safety assessment were focussed on delmopinol rather than CHX. Others were reported on a VAS scale. Treatment with $\mathrm{CHX}$ resulted according to patients in more staining of teeth and tongue than the other 2 mouthrinses. An anaesthetic sensation transient anaesthesia at the tip of the tongue in the oral mucosa was experienced more clearly by the patients in the delmopinol group than by those using placebo or $\mathrm{CHX}$

Results from the VAS (estimated from Figure 3):

-Taste: CHX 58 (SE 8), placebo 23 (SE 5), delmopinol 60 (SE 8)

-Staining of teeth/tongue: CHX 38 (SE 7), placebo 9 (SE 1), delmopinol 10 (SE 5)

-Clean mouth: CHX 29 (SE 9), placebo 33 (SE 7), delmopinol 30 (SE 8)

-Vesicles and/or ulcerations: CHX 9 (SE 1), placebo 8 (SE 1), delmopinol 7 (SE 0.5)

-Burning sensation: CHX 18 (SE 5), placebo 8 (SE 0.5), delmopinol 17 (SE 6)

-Transient anaesthetic sensation: CHX 21 (SE 9), placebo 10 (SE 4), delmopinol 38 (SE 9)

Declarations/conflicts of interest: none explicitly stated but first and last authors were employees of Biosurface AB, Malmo, Sweden

\section{Risk of bias}


Hase 1995 (Continued)

\section{Bias Authors'judgement Support for judgement}

Random sequence genera- Low risk tion (selection bias)
Quote: "Patients were allocated to treatment groups according to a computer-generated randomisation list, where treatment was balanced within blocks of 6 patients"

Comment: adequate method of random sequence generation

Allocation concealment $\quad$ Unclear risk
(selection bias)
Quote: "Packaging and labelling were carried out at an independent Clinical
Service Department"

Comment: it is not clear whether the assignment of subjects to groups was carried out at the independent Clinical Service Department

$\begin{array}{ll}\begin{array}{l}\text { Blinding of participants } \\ \text { and personnel (perfor- } \\ \text { mance bias) }\end{array} & \text { High risk } \\ \text { All outcomes } & \begin{array}{l}\text { Quotes: "double-blind" and "The various test solutions were dispensed in } \\ \text { identical } 200 \mathrm{ml} \text { amber glass bottles" and "The placebo was flavoured in order } \\ \text { to imitate the sensation of rinsing with the active solutions" }\end{array} \\ & \begin{array}{l}\text { Comment: the higher incidence of staining in the CHX group (plus worse taste, } \\ \text { greater transient anaesthetic sensation, and greater burning sensation in the } \\ \text { active groups) meant that participants could have worked out which group } \\ \text { they were in and this could have affected their oral health behaviours and } \\ \text { hence the outcomes. The direction of this potential bias is not clear }\end{array}\end{array}$

Blinding of outcome as- High risk sessment (detection bias) All outcomes
The study is reported to be "double-blind". There was 1 clinical examiner. Quote: "The split-mouth technique was used in the study in order to avoid interference between various assessments"

Comment: the authors do not report precisely who was blinded, but even if this includes the outcomes assessor he higher incidence of staining in the $\mathrm{CHX}$ group meant that they could not be adequately blinded and this could have affected the outcome assessment. The direction of this potential bias is not clear

Incomplete outcome data High risk
(attrition bias)

All outcomes
Losses to follow-up at 4 weeks: 3/42 (7\%). By group: placebo 3/21 (14.29\%). There were no losses from the $\mathrm{CHX}$ group. Reasons for losses by group: placebo, 1 did not meet the eligibility criteria and 2 took antibiotics during the trial

Comment: although reasons for attrition do not appear to be related to the particular interventions, the differential loss introduces the possibility that the results may be distorted

$\begin{array}{ll}\begin{array}{l}\text { Selective reporting (re- } \\ \text { porting bias) }\end{array} & \text { Low risk } \\ & \begin{array}{l}\text { Information on means and SEs are presented graphically. From correspon- } \\ \text { dence with the author/research team, additional information was obtained } \\ \text { on means and SEs which enabled calculation of SDs from SEs and inclusion of } \\ \text { these data in the meta-analyses }\end{array}\end{array}$

Other bias Unclear risk
No information is provided on intra- and interexaminer reproducibility.

Mean age was balanced across groups at baseline. No statistically significant demographic differences between the groups were recorded. Baseline BOP and mean plaque scores appear balanced but the statistical significance of the differences is not reported

\section{Study characteristics}

Methods Trial design: parallel (3 arms - 2 arms relevant to this review)


Hase 1998 (Continued)

Location: military regiment, Halmstad, Sweden

Number of centres: 1

Study duration: recruitment period is not stated but the duration of rinsing was 6 months

Participants

Participants: military conscripts and military staff (adult males) with gingivitis

Inclusion criteria: males aged at least 18 years; at least $25 \%$ bleeding sites of all gingival sites (6 sites per tooth) on BOP using a Florida-probe with a pressure of $0.25 \mathrm{~N}$ to the bottom of the pocket; minimum 16 healthy teeth without crowns, bridge-work, or ill-fitting dental restorations

Exclusion criteria: 4 or more teeth with cavities or pocket probing depth $5 \mathrm{~mm}$ or more; previous hypersensitive reactions to drugs; alcohol or drug addiction; severe liver or renal disease; psychiatric disorders; severe disability with multiple drug therapy; receiving antibiotics within 6 weeks of the prestudy visit; antiphlogistics (including sprays with corticosteroids) or anticholinergic drugs such as antiparkinson drugs or antidepressants

Baseline gingivitis: (\% sites BOP) Gp A: mean 42.7 (SE 2.7); Gp B: mean 47.3 (SE 2.6); Gp C: mean 43.9 (SE 2.7) (unpublished data)

Age at baseline (years): Gp A: mean 24 (SD 10); Gp B: mean 26 (SD 7); Gp C: mean 23 (SD 7)

Gender: males only

Number randomised: 140 (Table 2 page 748) (Gp A: 47; Gp B: 48; Gp C: 45)

Number evaluated (per protocol analysis): 100 (Gp A: 30; Gp B: 37; Gp C: 33)

Interventions

Comparison: $\mathrm{CHX}$ digluconate rinse versus delmopinol hydrochloride rinse ${ }^{\star}$ versus placebo rinse

Gp A ( $n=47)$ : CHX (0.2\% Hibitane Dental), $10 \mathrm{~mL}, 1 \mathrm{~min}$, twice daily

${ }^{*} \mathrm{Gp} \mathrm{B}(\mathrm{n}=48)$ : delmopinol hydrochloride $(0.2 \%)$, same routine. We excluded this arm from our risk of bias and analysis

Gp C ( $n=45)$ : same routine using placebo (same as Gp B but without delmopinol and sodium hydroxide)

Prophylaxis at baseline: supra- and subgingival scaling

$\mathrm{OHI}$ : participants were instructed not to change their normal mechanical tooth cleaning methods during the study

Partly supervised rinsing (mostly supervised but occasionally unsupervised due to military training)

Timing of rinsing in relation to toothbrushing: after toothbrushing

Postrinsing instructions: not reported

Outcomes

Gingivitis (gingival BOP using a Florida-probe with a pressure of $0.25 \mathrm{~N}$ to the bottom of the pocket (Gibbs 1988, Löe 1967)), measured at 3 and 6 months

Plaque (PI, TQH, Turesky 1970) measured at 3 and 6 months

Calculus (Volpe-Manhold Calculus Index, Volpe 1967), measured at 3 and 6 months

Extrinsic tooth staining (Staining Index of Shaw \& Murray 1977), measured at 3 and 6 months

Adverse events (transient anaesthetic sensation, taste modification, staining (teeth or tongue or both)) were recorded 
Hase 1998 (Continued)

Microbiological monitoring of plaque and salivary microflora was performed during the treatment period and also 3 months after the end of treatment for 65 patients - results (excluding BOP which is presented here) presented separately - not relevant to this review

\begin{tabular}{|c|c|}
\hline Funding & Not reported \\
\hline \multirow[t]{6}{*}{ Notes } & $\begin{array}{l}\text { Sample size calculation: based on BOP. The standard deviation was estimated to } 14 \text { and a difference of } \\
10 \% \text { units between placebo and delmopinol was considered worth detecting. Based on a power of } 95 \% \\
\text { at significance level of 5\%, } 50 \text { participants per group were required. Therefore this was not achieved }\end{array}$ \\
\hline & $\begin{array}{l}\text { Adverse effects: transient anaesthetic sensation in the oral mucosa especially at the tip of the tongue } \\
\text { in delmopinol and } \mathrm{CHX} \text { groups reached the same level of reporting at } 6 \text { months. Taste modification was } \\
\text { commonly reported in both the delmopinol and } \mathrm{CHX} \text { groups. Staining of teeth or tongue was common } \\
\text { in the } \mathrm{CHX} \text { group }\end{array}$ \\
\hline & Adverse effects at 6 months (Table 4 ) (\% participants experiencing the adverse effect): \\
\hline & local anaesthesia/hypoasthesia/parasthesia $\mathrm{CHX} 18 \%$, placebo $0 \%$, delmopinol \\
\hline & 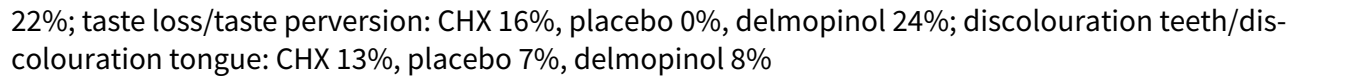 \\
\hline & $\begin{array}{l}\text { Declarations/conflicts of interest: nothing explicitly stated but first author was employee of Biosurface } \\
\text { AB, Malmo, Sweden }\end{array}$ \\
\hline
\end{tabular}

\section{Risk of bias}

Bias Authors' judgement Support for judgement

Random sequence genera- Low risk tion (selection bias)
Quote: "Patients were allocated to treatment groups according to a computer-generated randomisation list, where treatment was balanced within blocks of 6 patients"

Comment: adequate method of random sequence generation
Allocation concealment Unclear risk Quote: "Packaging and labelling were carried out at an independent Clinical (selection bias) Service Department"

Comment: it is not clear whether the assignment of subjects to groups was carried out at the independent Clinical Service Department

Blinding of participants High risk
and personnel (perfor-

mance bias)

All outcomes

\begin{abstract}
The study is reported to be "double-blind" and "The various test solutions were dispensed in identical $200 \mathrm{ml}$ amber glass bottles." The placebo was the same composition as the delmopinol rinse but without the delmopinol and sodium hydroxide. It is unclear who supervised the rinsing and if they were blinded to allocation of the rinse

Comment: the higher level of staining of the teeth and tongue in the $\mathrm{CHX}$ group (plus greater taste modification and transient anaesthetic sensation in the active groups) meant that participants could have worked out which group they were in and this could have affected their oral health behaviours and hence the outcomes. The direction of this potential bias is not clear
\end{abstract}

The study is reported to be "double-blind". Assessments were made by 5 trained dental hygienists who followed the same subjects throughout the treatment period

Comment: the authors do not report precisely who was blinded, but even if this includes the outcomes assessors the higher level of staining of the teeth and tongue in the $\mathrm{CHX}$ group meant that they could not be adequately blinded 
Hase 1998 (Continued)

and this could have affected the outcome assessment. The direction of this potential bias is not clear

Incomplete outcome data High risk (attrition bias)

All outcomes
At 6 months 29 participants are excluded from the analysis in the study arms of interest to this review but only 16 are reported as withdrawn from the study with reasons for withdrawal. Data presented here for loss to follow-up are based on the total lost to follow-up $(n=29)$

Attrition at 6 months 29/92 (31.5\%). By group: $\mathrm{CHX} \mathrm{17/47} \mathrm{(36.2 \% ),} \mathrm{placebo}$ $12 / 45$ (26.7\%). Reasons for loss to follow-up: CHX: adverse events/lack of cooperation, release from military service; placebo: adverse events/lack of cooperation, release from military service, moved away

Comment: very high attrition which may be related to type of mouthrinse and could feasibly introduce bias in the results

Selective reporting (re- Low risk
porting bias)

Information on means and SEs are presented graphically. From correspondence with the author, additional information was obtained on means and SEs which enabled calculation of SDs from SEs and inclusion of these data in the meta-analyses

Other bias Unclear risk

Assessments were made by 5 trained dental hygienists who followed the same subjects throughout the treatment period. A calibration session for all assessments was performed before the start of treatment but the results of the calibration (Inter- and intraexaminer reliability) are not reported

Mean age was balanced across groups at baseline. No statistically significant demographic differences between the groups were recorded. Baseline BOP and mean plaque scores appear balanced but the statistical significance of the differences is not reported

Hoffmann 2001

\title{
Study characteristics
}

Trial design: parallel (5 arms - 4 arms relevant to this review)
Location: subjects were recruited from the University of Technology, Dresden. The setting was the Uni-
versity Periodontal Clinic
Number of centres: 1
Study duration: the period of recruitment is not stated. Study duration and duration of rinsing was 6
months after a run in period of 2 weeks

Participants

\begin{abstract}
Participants: medical students
Inclusion criteria: quote: "exhibiting good oral hygiene... Inclusion criteria were at least 20 teeth, ginigival index $(\mathrm{Gl})<1.5$, and no periodontitis"

Exclusion criteria: quote: "Exclusion criteria were allergic reactions to any of the components of any mouth rinse, antibiotic/antiphlogistic medication in the previous 8 weeks, dental-treatment needs (e.g. due to acute carious lesions), pregnancy or any kind of systemic diseases"
\end{abstract}

Baseline gingivitis: not reported but had to be $<1.5$ for inclusion in the study

Age at baseline (years): mean age is not reported. Age range 18-36 years

Gender: males 28/81 (34.6\%), females 53/81 (65.4\%) 
Hoffmann 2001 (Continued)

Number randomised: 81 (CHX 0.10\% 16, CHX 0.06\% 17, CHX 0.06\%/F 17, ASF 16, control 15)

Number evaluated: 73 (CHX 0.10\% 14, CHX 0.06\% 16, CHX 0.06\%/F 17, ASF 15, control 11)

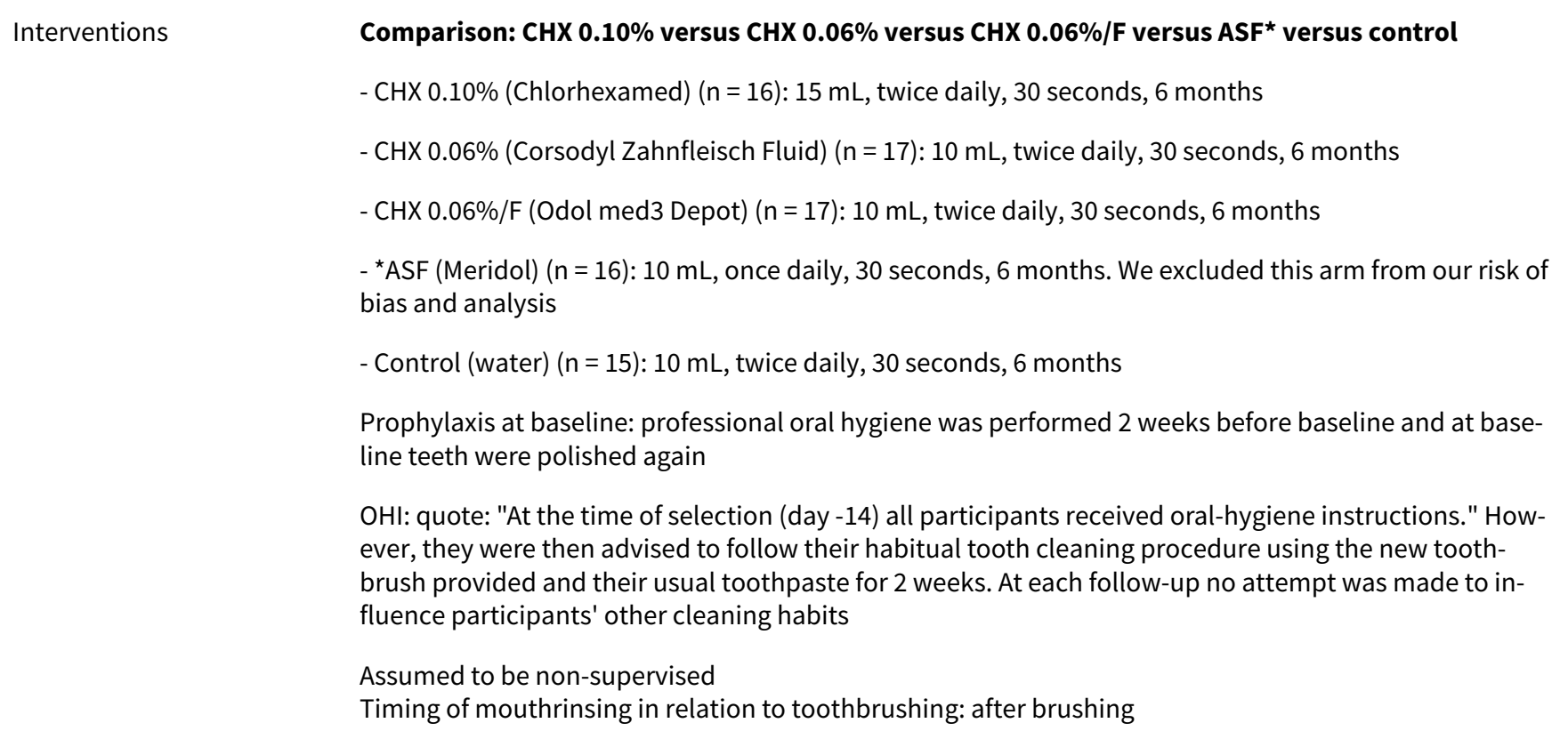

\section{Outcomes}

Gingivitis (gingival inflammation: GI, Löe \& Silness 1967); plaque (PI, Silness \& Löe 1964); tooth staining (Discolouration Index (Lang \& Räber 1981) assessed at day -14, at baseline and at 1, 2, 3, and 6 months

\begin{tabular}{ll}
\hline Funding & Not reported \\
\hline Notes & Sample size calculation: not reported
\end{tabular}

Adverse effects: Quotes: "One subject in the control group, however, had to be taken out because of leukoplakia..., no dropout occurred due to side effects of any of the mouthwash preparations" and in relation to tooth staining "Except the $0.06 \% \mathrm{CHX}$, all test solutions showed significantly higher DI scores when compared to the control... However from a clinical point of view, regarding the DI, no important differences between the active groups were seen" and "the $0.1 \%$ resulted in stronger staining than the ASF and the $0.06 \%$ CHX.."

Declarations/conflicts of interest: none stated

\section{Risk of bias}

Bias Authors' judgement Support for judgement

Random sequence genera- Unclear risk tion (selection bias)
Quote: "According to their clinical parameters... and their sex, the 85 panellists were distributed at random and stratified to the 5 test groups"

Comment: no description of the method of random sequence generation

Allocation concealment Unclear risk No information is provided

(selection bias)

$\begin{array}{ll}\begin{array}{l}\text { Blinding of participants } \\ \text { and personnel (perfor- }\end{array} & \text { High risk } \\ \begin{array}{ll}\text { mance bias) } & \text { The authors report this to be a "blind clinical study" } \\ \text { All outcomes } & \text { Quote: "The different test solutions were presented in similar bottles. However } \\ & \text { each participant got his/her specific information regarding the use of mouth- } \\ \text { washes from an independent person not involved in the examinations" }\end{array}\end{array}$


The control rinse was tap water which the authors recognise as an issue (page 93). Quote: "After investigation it was revealed that some of the participants of the control group did not understand the scientific sense of rinsing with tap water"

The $0.1 \% \mathrm{CHX}$ group rinsed with $15 \mathrm{~mL}$ and the other groups rinsed with $10 \mathrm{~mL}$

In relation to tooth staining, quotes: "Except the $0.06 \% \mathrm{CHX}$, all test solutions showed significantly higher DI scores when compared to the control.. However from a clinical point of view, regarding the DI, no important differences between the active groups were seen" and "the $0.1 \%$ resulted in stronger staining than the ASF and the $0.06 \%$ CHX.."

Comment: the control group cannot be considered blind. The higher level of staining in the active groups (ASF and CHX) meant that participants could have worked out that they were in 1 of the active mouthrinse groups and this could have affected their oral health behaviours and hence the outcome. The direction of this potential bias is unclear

\section{Blinding of outcome as- High risk} sessment (detection bias) All outcomes

The authors report this to be a "blind clinical study"

Quote: "One investigator assessed PII and GI, while another estimated DI during the study"

In relation to tooth staining, quotes: "Except the $0.06 \% \mathrm{CHX}$, all test solutions showed significantly higher DI scores when compared to the control... However from a clinical point of view, regarding the DI, no important differences between the active groups were seen" and "the $0.1 \%$ resulted in stronger staining than the ASF and the $0.06 \%$ CHX.."

Comment: the higher level of staining in the active groups meant that the outcome assessor could not be adequately blinded and this could have affected the outcome assessment. Even though a separate examiner was used to record $\mathrm{PI}$ and $\mathrm{GI}$ and the $\mathrm{DI}$ the examiners recording the $\mathrm{PI}$ and $\mathrm{GI}$ would still be aware of the increased tooth staining. The direction of this potential bias is not clear

Incomplete outcome data High risk (attrition bias)

All outcomes
85 individuals were randomised to groups but only 81 were "included in the study". The total number originally allocated to each group (from the 85 ) is not reported therefore 85 is used as the denominator for the calculation of overall attrition and 81 is used as the denominator for calculating the attrition in each group

Attrition at 6 months $8 / 85$ (9.4\%). Attrition by group: $\mathrm{CHX} \mathrm{0.1 \%} \mathrm{2/16} \mathrm{(12.5 \% ),}$ $\mathrm{CHX} 0.06 \% 1 / 17$ (5.9\%), CHX 0.06\%/F 0\%, ASF 1/16 (6.3\%), control 4/15 $(26.7 \%)$. Reasons for loss to follow-up: quote: "One subject in the control group.. had to be taken out because of leukoplakia; two others did not show up at the last examination. Another two losses were in the $0.1 \% \mathrm{CHX}$ group, but these cases were not due to any adverse effects. Furthermore, two losses occurred because of antibiotic medication during this long experimental time"

Comment: there were differential losses between the groups with a notably higher loss in the control group. This is conjunction with the loss of 4 participants before the study started indicate an increased risk of bias

Results for all outcomes were reported in text at 3 and 6 months and at all time points in box plot diagrams. Median values only were reported and no information on the variance of the scores was provided

Selective reporting (re- High risk
porting bias)

Other bias
Unclear risk 
Hoffmann 2001 (Continued)

There were more females than males in each group at baseline ranging from $66 \%$ in the control group to $70 \%$ in both $0.06 \%$ CHX groups. It is reported that there were no differences between the groups at baseline for the 3 clinical parameters

No other baseline data are reported by group

Jayaprakash 2007

\section{Study characteristics}

\section{Methods}

Trial design: parallel 3 arms

Location: Sri Laksmi Narasimha High School, Bangalore, India

Number of centres: 1

Study duration: the recruitment period is not reported. Study duration and duration of rinsing was 6 months

\section{Participants}

Participants: school children (from Sri Laksmi Narasimha High School) in Bangalore city

Inclusion criteria: quote: "Inclusion criteria involved written informed consent from parents, good general health of children, a minimum of 12 gradable teeth, agreement to delay any elective dental treatment, including oral prophylaxis during the course of the study, and the agreement to comply with the study visits and procedures"

Exclusion criteria: quote: "Exclusion criteria involved antibiotic therapy, history of early-onset periodontitis, acute necrotizing ulcerative gingivitis, gross oral pathology, treatment for cancer or seizure disorders and conditions that interfered with the examination procedures"

Baseline gingivitis: (GI, Löe \& Silness 1963) CHX 0.0536, CHX/NaF 0.0562, placebo 0.0609. SD not reported

Age at baseline (years): mean age not reported. Age range 13-16 years

Gender: not reported

Number randomised: 150 (numbers allocated to each group not reported, assumed 50 in each group at baseline)

Number evaluated: not reported

\section{Comparison: $\mathrm{CHX}$ rinse versus $\mathrm{CHX} / \mathrm{NaF}$ rinse versus placebo rinse}

Gp A ( $n$ = assumed to be 50) CHX 0.05\%: $10 \mathrm{~mL}$, once daily, 6 months

$\mathrm{Gp} \mathrm{B} \mathrm{(} \mathrm{n}=$ assumed to be 50) $\mathrm{CHX} / \mathrm{NaF}$ 0.05\%: $10 \mathrm{~mL}$, once daily, 6 months

Gp C ( $n$ = assumed to be 50) placebo: $10 \mathrm{~mL}$, once daily, 6 months

Rinse duration not reported

Prophylaxis at baseline: quote: "...no oral prophylaxis ... were given"

OHI: quote: "no ...oral hygiene instructions were given"

Supervised rinsing

Timing of mouthrinsing in relation to toothbrushing: not stated, however rinsing was done in school so likely at a different time to toothbrushing 
Jayaprakash 2007 (Continued)

Postrinsing instructions: instructed not to eat or drink anything for an hour after using the mouthrinse

Outcomes Gingivitis (gingival inflammation: GI, Löe \& Silness 1963) and plaque (PI, Silness \& Löe 1964), assessed after 1,3 , and 6 months

\begin{tabular}{ll}
\hline Funding & Not reported \\
\hline Notes & Sample size calculation: not reported \\
& Adverse effects: not reported \\
& Declarations/conflicts of interest: not reported \\
& Gingivitis data for Gp A and Gp B were combined for the main analysis
\end{tabular}

\section{Risk of bias}

\section{Bias}

\section{Authors' judgement Support for judgement}

Random sequence genera- Unclear risk tion (selection bias)
Quote: "The students were randomly numbered 1-150 and the mouthwash samples were numbered randomly 1-150 by the mouthrinse manufacturer.... the students who were assigned with particular numbers were provided with the mouthwash with the same number"

Comment: no description of the method of random sequence generation
Allocation concealment Low risk (selection bias)
Quote: "The students were randomly numbered 1-150 and the mouthwash samples were numbered randomly $1-150$ by the mouthrinse manufacturer.... the students who were assigned with particular numbers were provided with the mouthwash with the same number"

Comment: coding of mouthrinse samples done by manufacturer. We consider it unlikely that participants or investigators enrolling participants could foresee assignments

Blinding of participants Unclear risk
and personnel (performance bias)

All outcomes

The study is reported to be "double-blind". The investigator supervised the mouthrinsing performed by the students daily. No information is provided on the composition of the placebo. Tooth staining is not mentioned
Comment: tooth staining is likely to have been noticeable in both $\mathrm{CHX}$ groups compared to placebo during this 6-month study and in this situation participants could have worked out which group they were in and this could have affected their oral health behaviours and hence the outcome. However, tooth staining is not reported and there is not enough information upon which to base a judgement

Blinding of outcome as-
sessment (detection bias)
All outcomes

The study is reported to be "double-blind". The investigator supervised the mouthrinsing performed by the students daily. Tooth staining is not mentioned

Comment: tooth staining is likely to have been noticeable in both $\mathrm{CHX}$ groups compared to placebo during this 6-month study and in this situation the outcome assessor could have worked out which individuals were in the placebo group and not be adequately blinded. However, tooth staining is not reported and there is not enough information upon which to base a judgement
Incomplete outcome data
Unclear risk
The number in each group at the start and end of the study is not reported (attrition bias)

All outcomes 
Jayaprakash 2007 (Continued)

Selective reporting (re- High risk Mean scores for gingivitis and plaque are reported without any measure of porting bias) variance of the mean scores. We estimated the SD for gingivitis (measured using the GI) as the median of the SDs in the placebo/control groups from similar studies that also used the Gl at 6 months so that we could include the gingivitis data in the meta-analyses. Furthermore, adverse effects including tooth staining are considered to be important outcomes but are not reported

Other bias Unclear risk Outcomes were assessed by 1 individual. Details of training and intraexaminer reliability are not reported

Baseline demographic data for the participants are not reported

Jenkins 1993

\section{Study characteristics}

Methods Trial design: parallel, 2 arms

Location: dental clinic, University of Wales College of Medicine, Cardiff, Wales, UK

Number of centres: 1

Study duration: recruitment period not stated. Study duration and the duration of rinsing was 6 weeks

Participants Participants: adults with gingivitis (employees of the University of Wales College of Medicine and South Glamorgan Area Health Authority)

Inclusion criteria: between 18 and 60 years old; minimum 16 natural teeth; minimum Löe \& Silness GI score of 0.5

Exclusion criteria: wearing dental prosthesis or appliance; advanced restorative dental work; medical or pharmacotherapy history affecting the study

Baseline gingivitis: (Löe \& Silness GI) Gp A: mean 0.89 (SD 0.24); Gp B mean 0.88 (SD 0.21) - means read from graph (Figure 3)

Age at baseline (years): 18 to 60 (not reported by group)

Gender: not reported

Number randomised: 102 (Gp A: 51; Gp B: 51)

Number evaluated: 99 (Gp A: 49; Gp B: 50)

\section{Comparison: (CHX gluconate + sodium fluoride (NaF) rinse) versus (placebo rinse (without $\mathrm{CHX}$ or fluoride))}

Gp A ( $\mathrm{n}=51): \mathrm{CHX}(0.12 \%)$ plus NaF (100 ppm F), $15 \mathrm{~mL}, 1$ min, twice daily (morning and evening)

$\mathrm{Gp} \mathrm{B}(\mathrm{n}=51)$ : same routine using placebo

Prophylaxis at baseline: none but could request and receive at any time a polishing of the anterior teeth if they perceived a cosmetic problem

OHI: none, but all participants received same toothbrush and fluoride toothpaste but the use of any oral hygiene products other than those supplied was not permitted

Non-supervised rinsing

Timing of mouthrinsing in relation to toothbrushing: not reported 
Jenkins 1993 (Continued)

Postrinsing instructions: not reported

Outcomes Gingivitis (gingival inflammation: GI Löe \& Silness 1963 "modified to standardize the probing force, to elicit bleeding, using a constant pressure probe set at $25 \mathrm{~g}$ "); plaque (PI, TQH, Turesky 1970); stain area (modification of Shaw \& Murray stain index 1977, stain severity graded according to colour (0 to 5 scale)), all measured at 6 weeks Problems associated with the use of rinses, particularly due to staining were elicited by questionnaire

\begin{tabular}{ll}
\hline Funding & Quote: "The authors are grateful to Smith Kline Beecham for supporting this study..." \\
\hline Notes & Sample size calculation: not reported \\
& Adverse effects: quotes: "Mucosal erosions were clinically apparent in two subjects using the active \\
rinse and a further 2 subjects in this group reported mucosal soreness" and "soreness tingling and taste \\
disturbance were more commonly reported for the active rinse and the difference between the groups \\
reached statistical significance for taste disturbance $(P<0.05)$. Significantly more subjects $(49 \%)$ using \\
the active rinse were noted to have staining than using the control rinse $(4 \%)(P<0.001)$. Also $82 \%$ of \\
the active group reported staining compared with $14 \%$ in the control group $(P<0.001) .2 / 3$ of those re- \\
porting staining on active treatment classed it as quite bad or unacceptable, compared to none on the \\
control rinse $(P<0.001)$. In response to the question of liking the taste of the mouthrinse coded as yes $=$ \\
1, equivocal $=1.5$ and no $=2$ there was a clear preference for the control rinse $(P<0.001) "$ \\
Declarations $/$ conflicts of interest: not reported
\end{tabular}

\section{Risk of bias}

Bias Authors' judgement Support for judgement

Random sequence genera- Unclear risk tion (selection bias)

Allocation concealment Low risk
(selection bias)

Quote: "..volunteers randomly allocated to the active or control rinse"

Comment: no description of the method of random sequence generation

Quote: "..volunteers were dispensed the allocated rinse through the Dental Hospital Pharmacy where a sealed code-breaker was kept"

Comment: we consider it unlikely that participants and investigators enrolling participants could foresee assignment

\begin{tabular}{|c|c|c|}
\hline $\begin{array}{l}\text { Blinding of participants } \\
\text { and personnel (perfor- } \\
\text { mance bias) } \\
\text { All outcomes }\end{array}$ & High risk & $\begin{array}{l}\text { The study is reported to be "double-blind" } \\
\text { The control rinse was identical to the test rinse "except that it contained nei- } \\
\text { ther chlorhexidine or fluoride" }\end{array}$ \\
\hline
\end{tabular}

Quote: "Significantly more subjects (49\%) using the active rinse were noted to have staining than using the control rinse $(4 \%)(P<0.001) "$

Comment: the higher level of staining in the $\mathrm{CHX}$ group meant that participants could have worked out which group they were in and this could have affected their oral health behaviours and hence the outcome. The direction of this potential bias is not clear

Blinding of outcome assessment (detection bias) All outcomes
High risk

The study is reported to be "double-blind". Quote: "Significantly more subjects $(49 \%)$ using the active rinse were noted to have staining than using the control rinse $(4 \%)(P<0.001) "$

Comment: the authors do not report precisely who was blinded, but even if this includes the outcomes assessors the higher incidence of staining in the active group meant that they could not be adequately blinded and this could have affected the outcome assessment. The direction of this potential bias is not clear 
Jenkins 1993 (Continued)

Incomplete outcome data Low risk Attrition after 6 weeks was 3/102 (2.9\%). Attrition by group: $2 / 51$ (3.9\%) CHX (attrition bias) $0.12 \%, 1 / 51(2.0 \%)$ control. Reasons for losses not broken down by group: All outcomes quote: "..three subjects withdrew, 1 for reasons of taste of the active rinse and 2 for reasons unrelated to treatment"

Comment: losses are very low and not considered sufficient to have any effect on the results

\begin{tabular}{lll}
$\begin{array}{l}\text { Selective reporting (re- } \\
\text { porting bias) }\end{array}$ & Low risk & $\begin{array}{l}\text { Mean gingival and plaque data, stain area and intensity are reported graph- } \\
\text { ically with SD (as a number on the graph). Mean scores were estimated from } \\
\text { graphs. Stain area only was used in the meta-analysis }\end{array}$ \\
\hline Other bias & Unclear risk & $\begin{array}{l}\text { No information is provided on number of examiners, examiner training or in- } \\
\text { tra- or interexaminer reproducibility so it is unclear whether or not there was a } \\
\text { risk of differential diagnostic activity. Baseline participant characteristics (age, } \\
\text { gender, etc) are not reported. There was no significant difference between the } \\
\text { CHX and control groups for plaque, gingivitis, proportion of smokers and tooth } \\
\text { staining at baseline }\end{array}$
\end{tabular}

Jose 2015

\section{Study characteristics}

Methods

Trial design: parallel group ( 3 arms)

Location: Manchester and Wirral, UK, Clinical Research Facility

Number of centres: 2

Study duration: the recruitment period is not stated. Study duration and duration of rinsing was 6 weeks

\section{Participants}

Participants: healthy adults recruited from the sites' databases and via advertisements

Inclusion criteria: at the screening visit, subjects were asked to brush as they normally would for 1 timed minute and to expectorate into a white cup. Evidence of blood in the expectorant or bleeding while brushing were the initial inclusion criteria. Additional inclusion/exclusion criteria used were: $\geq 16$ permanent, gradable teeth, including 4 molars (protocol amendment 3: changed from 20 gradable teeth); and $\geq 20$ or more bleeding sites at the baseline examination

Exclusion criteria: women who were pregnant, lactating or those of child bearing potential not practising a reliable method of contraception were excluded from the study. Subjects who were taking antibiotics within 2 weeks before the screening visit or throughout the study were excluded, as were those on concomitant medication that, in the opinion of the investigator, might interfere with the outcome of the study. Other than having mild to moderate gingivitis, subjects were to be in good oral health with no active caries, no heavy calculus deposits and no more than 5 periodontal pockets measuring $\geq 5 \mathrm{~mm}$ in depth (protocol amendment 3: changed from more than 3 pockets $>/-5 \mathrm{~mm}$ in depth)

Baseline gingivitis: (GI, Löe \& Silness) Gp A: mean 1.24 (SE 0.009); Gp B: mean 1.24 (SE 0.009); Gp C: mean 1.24 (SE 0.01)

Age (years): Gp A: mean 37.8; Gp B: mean 36.2; Gp C: 36.5. Range 18-63 years. Gp A: range 18-62; Gp B: range 18-63; Gp C: range: $18-56$

Gender: overall: male 85 (26\%), female 239 (74\%). Gp A: male 23 (21\%), female 85 (79\%); Gp B: male 30 (28\%), female 79 (73\%); Gp C: male 32 (30\%), female $75(70 \%)$

Number randomised: 324 (Gp A: 108; Gp B: 109; Gp C: 107) 
Jose 2015 (Continued)

Number evaluated (ITT): 319 (Gp A: 105; Gp B: 109; Gp C: 105)

Interventions

Comparison: (CHX rinse with alcohol + standard fluoride toothpaste) versus ( $\mathrm{CHX}$ rinse alcohol-free + standard fluoride toothpaste) versus (brushing alone with standard fluoride toothpaste)

$\mathrm{Gp} \mathrm{A}(\mathrm{n}=108)$ : $\mathrm{CHX}$ with alcohol $0.2 \%, 10 \mathrm{~mL}$, twice daily, 1 min, 6 weeks

Gp B ( $n=109):$ CHX alcohol-free 0.2\%, $10 \mathrm{~mL}$, twice daily, 1 min, 6 weeks

Gp C ( $=107)$ : brushing alone (standard toothbrush and toothpaste)

All 3 groups brushed twice daily with full brush head of toothpaste for 1 timed minute

Prophylaxis at baseline: quote: "A complete dental prophylaxis, which included flossing to ensure removal of all plaque, was performed by an appropriately trained professional at the baseline visit or on a separate visit within 7 days of baseline"

OHI: the first use of the product/toothbrushing (1 timed minute) was supervised but it is not clear whether the participants received instructions on brushing technique. We assumed not

Rinsing was unsupervised (apart from the first use of the rinse)

Timing of mouthrinsing in relation to toothbrushing: quote: "subjects .... brushed their teeth with a full brush head of toothpaste for one timed minute, rinsed their mouth with water then waited for five timed minutes. After five minutes, they swished with $10 \mathrm{ml}$ of their assigned mouthwash (mouthwash groups only) for a timed one minute followed by expectorating"

Duration of rinsing: 6 weeks

Outcomes

Gingivitis (gingival inflammation: GI, Löe \& Silness 1963; gingival bleeding: Gingival Severity Index); plaque (Soparkar's modification of the TQH, Turesky 1970, Lobene 1982); oral soft tissue examination and $\mathrm{CHX}$ pharmacokinetic variables (from saliva samples) - not relevant to this review at 6 weeks follow-up

Funding

The study and writing support for the manuscript were funded by GSK Consumer Healthcare

Notes The study protocol was registered at Clinicaltrials.gov (NCT01751178) and at www.gsk-clinicalstudyregister.com/study/RH01561\#ps

Sample size calculation: not reported

Adverse effects: quote: "A total of 338 TEAEs (treatment emergent adverse events) were recorded among 174 (53.7\%) of subjects (Table 4a). A large number of TRAEs (treatment related adverse events) were recorded in the two chlorhexidine groups where $27.8 \%$ of subjects in the $0.2 \% \mathrm{CHX}$-alcohol group and $24.8 \%$ in the $0.2 \% \mathrm{CHX}$-alcohol-free group reported at least one TRAE compared to only $3.7 \%$ in the brushing alone group. The most commonly reported TRAEs were coated tongue, glossodynia, oral paraesthesia, ageusia, dry mouth, oral hypoaesthesia and dysgeusia (Table $4 \mathrm{~b}$ ). There was one serious adverse event, not related to study product and one severe event (oral paresthesia) in the $0.2 \% \mathrm{CHX}$-alcohol group which did resolve. The subject withdrew from the study due to the event. A second subject withdrew from the study due to a non-TRAE"

Smoking status: overall smoker 43 (13\%), non-smoker 281 (87\%). Gp A: smoker 14 (13\%), non-smoker 94 (87\%); Gp B: smoker 15 (14\%), non-smoker 94 (86\%); Gp C: smoker 14 (13\%), non-smoker 93 (87\%)

Declarations/conflicts of interest: none stated, however the authors of the study are either employees of GSK Consumer Healthcare or employees of Intertek Clinical Research Services, Wirral and Manchester, UK which has received funding from GSK Consumer Healthcare

Groups A and B were combined in the main analysis

\section{Risk of bias}


Jose 2015 (Continued)

\section{Bias Authors' judgement Support for judgement}

Random sequence genera- Unclear risk tion (selection bias)
Quote: "Subjects were then stratified, according to the study site (Manchester or Wirral, UK), baseline number of bleeding sites and smoking status, and randomised into one of three treatment groups"

Comment: no description of the method of random sequence generation

Allocation concealment $\quad$ Unclear risk information is provided
(selection bias)

(selection bias)

Blinding of participants High risk
and personnel (perfor-

The study is described as "examiner-blinded". All participants could not be and personnel (perforblinded due to the design of the study which included a 'brushing alone' group

mance bias)

All outcomes

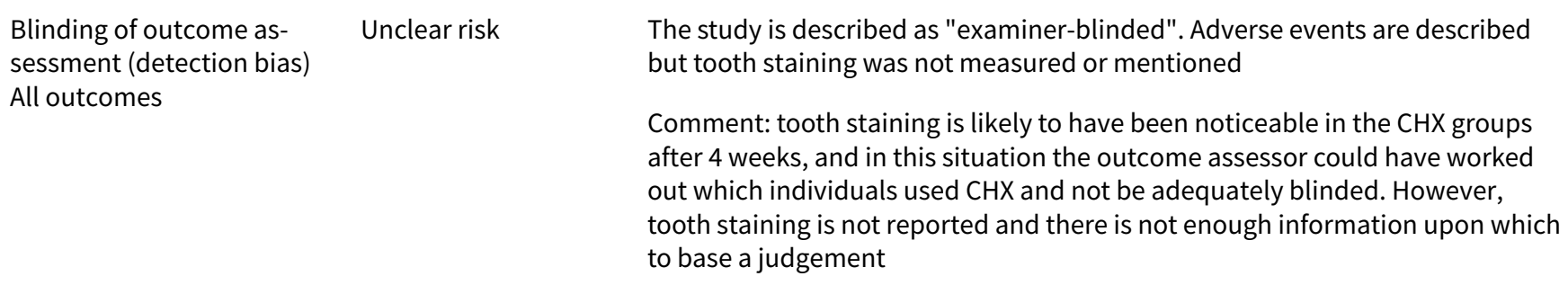

Incomplete outcome data Low risk
(attrition bias)

Attrition (ITT rather than PP): 5/324 (1.5\%). Gp A: 3/108 (2.8\%), Gp B: no losses, All outcomes Gp C: 2/107 (1.9\%). Reasons for loss to follow-up: Gp A: 2 subjects with adverse events and 1 withdrew consent; Gp C: 2 subjects withdrew consent

Comment: we do not believe the losses described could pose a risk of bias

\begin{tabular}{ll}
\hline $\begin{array}{l}\text { Selective reporting (re- } \\
\begin{array}{l}\text { porting bias) } \\
\text { High risk }\end{array}\end{array}$ & $\begin{array}{l}\text { All outcome stated in the methods section are described fully in the results. } \\
\text { Tooth staining is considered to be an important side effect of CHX mouthrins- } \\
\text { ing but is not reported }\end{array}$
\end{tabular}

\begin{tabular}{ll}
\hline Other bias & Low risk \\
& $\begin{array}{l}\text { Quote: "All assessments of GI were performed by a single, experienced examin- } \\
\text { er." Repeat assessment of PI were performed and showed excellent agreement } \\
\text { (kappa value for PI } 0.891(95 \% \mathrm{Cl} 0.884 \text { to }-0.889) .\end{array}$
\end{tabular}

Baseline demographics and \% bleeding sites at baseline appear balanced (Table 1). Mean Gingival Severity Index, GI and PI appear similar at baseline

\section{Study characteristics}

Trial design: parallel, 2 arms
Location: Department of Oral Medicine and Periodontology, The London Hospital Medical College, UK
Number of centres: 1
Study duration: recruitment period is not stated. Study duration and duration of rinsing was 8 weeks

Participants Participants: adults with gingivitis

Inclusion criteria: over 18 years old; minimum 20 natural teeth; minimum Community Periodontal Index of Treatment Needs (CPITN) score of greater than 1 in at least 2 sextants but less than 4 in any 
Joyston-Bechal 1993 (Continued)

sextant. This method of selection ensured that all subjects showed signs of gingivitis but no pockets greater than $5.5 \mathrm{~mm}$ in depth were present

Exclusion criteria: medical history affecting the study; pregnancy or lactation; open carious lesions; partial dentures; orthodontic banding; current periodontal treatment; mentally or physically handicapped with impaired dexterity

Baseline gingivitis: not reported

Age at baseline (years): not reported. Mean age of completers at baseline (years): Gp A: mean 30.5 (SE 2.5); Gp B: mean 32.8 (SE 2.9)

Gender (completers): overall: male 38.46\%; female 61.54\%. Gp A: male 6 (31.6\%), female 13 (68.4\%); Gp B: male 9 (45.0\%), female $11(55.0 \%)$

Number randomised: 47 (not reported by group)

Number evaluated: 39 (Gp A: 19; Gp B: 20)

(The text of the paper indicates that there were 20 individuals in the test group and 19 in the control group but Table 1 reports 19 individuals in the test group and 20 in control group. We have extracted the data presented in Table 1)

Interventions

Comparison: (CHX + sodium fluoride ( $\mathrm{NaF}$ ) rinse) versus (placebo rinse (similar colour and flavour but without $\mathrm{CHX}$ or fluoride))

Gp A ( $n=19$ analysed): $\mathrm{CHX}(0.05 \%)+0.05 \% \mathrm{NaF}(500 \pm 25$ ppm NaF), $10 \mathrm{~mL}, 30$ seconds, twice daily

$\mathrm{Gp} \mathrm{B}(\mathrm{n}=20$ analysed): same routine using placebo

Note: all subjects received the control mouthrinse for a run in period of 10 days to allow familiarisation with the requirements of the study

Prophylaxis at baseline: all teeth were scaled and polished on the first visit after assessments

OHI: subjects were asked to follow their routine dental hygiene practices, no oral hygiene instructions were given. All participants received the same toothbrush and fluoride toothpaste

Non-supervised rinsing

Timing of mouthrinsing in relation to toothbrushing: after brushing with toothpaste and rinsing with water

Postrinsing instructions: not reported

Outcomes

Gingivitis (gingival bleeding - Cowell 1975 with the following modification: the periodontal probe was inserted into the periodontal pockets to a depth of only 1-2 $\mathrm{mm}$ and not to the base of the pocket); plaque (PI, Silness \& Löe 1964); degree of staining (Lobene Stain Index 1968), all measured at 8 weeks

\begin{tabular}{|c|c|}
\hline Funding & Quote: "We are most grateful to Johnson and Johnson Limited for financial support for this study" \\
\hline \multirow[t]{4}{*}{ Notes } & Sample size calculation: not reported \\
\hline & $\begin{array}{l}\text { Adverse effects: not reported apart from tooth staining. The active rinse group had more staining than } \\
\text { the control group at baseline before the scale and polish and more staining than the control at the end } \\
\text { of the study }\end{array}$ \\
\hline & $\begin{array}{l}\text { Smokers: by group: Gp A: } 5 / 19 \text { ( } 26 \% \text { of completers); Gp B: } 7 / 20 \text { ( } 35 \% \text { of completers) (differences be- } \\
\text { tween the groups are not statistically significant) }\end{array}$ \\
\hline & Declarations/conflicts of interest: not reported \\
\hline
\end{tabular}

\section{Risk of bias}


Joyston-Bechal 1993 (Continued)

Bias Authors' judgement Support for judgement

\begin{tabular}{ll}
\hline $\begin{array}{l}\text { Random sequence genera- } \\
\text { tion (selection bias) }\end{array}$ & Quote: "randomised into test and control groups" \\
& Comment: no description of the method of random sequence generation
\end{tabular}

\begin{tabular}{ll}
\hline $\begin{array}{l}\text { Allocation concealment } \\
\text { (selection bias) }\end{array}$ & $\begin{array}{l}\text { Quote: "The bottles were randomly numbered so that neither examiners or } \\
\text { subjects were aware of the distribution of test or placebo solutions" }\end{array}$ \\
& $\begin{array}{l}\text { Comment: not enough information is provided. It is not clear how the alloca- } \\
\text { tion sequence was concealed before participants were randomised to groups }\end{array}$
\end{tabular}

\begin{tabular}{|c|c|c|}
\hline $\begin{array}{l}\text { Blinding of participants } \\
\text { and personnel (perfor- } \\
\text { mance bias) } \\
\text { All outcomes }\end{array}$ & High risk & $\begin{array}{l}\text { The authors describe the study as "double-blind" } \\
\text { Quotes: "The bottles were randomly numbered so that neither examiners or } \\
\text { subjects were aware of the distribution of the test or placebo solutions" and } \\
\text { "The placebo solution was of similar colour and flavour but with no active } \\
\text { components" and "Test and placebo solutions were supplied... in identical } \\
\text { bottles.." Baseline assessments of staining were completed before the scale } \\
\text { and polish and there was a statistically significant difference between the } \\
\text { groups at baseline with the test group having a higher mean staining score at } \\
\text { baseline and at the end of the study. The authors state that "subjects and ex- } \\
\text { aminers were unaware of any staining" but it is not clear how this information } \\
\text { was obtained }\end{array}$ \\
\hline
\end{tabular}

Comment: the active rinse group had more staining than the control group at baseline before the scale and polish and more staining than the control group at the end of the study. The higher incidence of staining in the active group meant that participants could have worked out which group they were in and this could have affected their oral health behaviours and hence the outcome. The direction of this potential bias is not clear

$\begin{array}{ll}\text { Blinding of outcome as- } & \text { High risk } \\ \text { sessment (detection bias) } & \end{array}$

All outcomes

Quotes: "..double-blind study.." and "The bottles were randomly numbered so that neither examiners or subjects were aware of the distribution of the test or placebo solutions" and "Test and placebo solutions were supplied... in identical bottles.." Baseline assessments of staining were completed before the scale and polish and there was a statistically significant difference between the groups at baseline with the test group having a higher mean staining score at baseline and at the end of the study. The authors state that "subjects and examiners were unaware of any staining" but it is not clear how this information was obtained

Comment: the authors do not report precisely who was blinded, but even if this includes the outcomes assessors the higher level of staining in the active group meant that the outcome assessors could not be adequately blinded and this could have affected the outcome assessment. The direction of this potential bias is not clear Incomplete outcome data High risk
(attrition bias)

All outcomes
Attrition after 8 weeks was 8/47 (17.0\%). Losses not broken down by group and reasons for losses not reported

Comment: assuming the missing participants in 1 group had a higher mean (e.g. gingivitis score) than those in the other group, as the attrition rate increased, so would the mean difference between groups. Also, although attrition was not reported by group, it could be associated with the outcomes
Selective reporting (re- High risk
porting bias)
Mean scores are reported graphically with a measure of variance (assumed to be SD) as a bar on the graphs. Mean scores and SDs were estimated from graphs so that these data could be included in meta-analyses. Apart from tooth staining, adverse effects are not mentioned 
Joyston-Bechal 1993 (Continued)

Other bias Low risk
Outcome assessment at the initial visit and the final visit were by different examiners but their interexaminer reproducibility is reported and is adequate. There was a statistically significant difference between the groups at baseline with the test group having a higher mean staining score at baseline and at the end of the study. No other apparent sources of bias were identified

\section{Study characteristics}

Methods Trial design: parallel (3 arms - 2 arms are relevant to this review)

Location: Berne, Switzerland. University Department of Periodontology and Fixed Prosthodontics Number of centres: 1

Study duration: the recruitment period is not stated but the duration of rinsing is 6 months

Participants

Participants: males and females from a telecommunication company in Berne, Switzerland

Inclusion criteria: quote: "Inclusion criteria were: 18 years old and above, with $\geq 25 \%$ of all gingival sites (six sites around each tooth) with bleeding upon probing (BOP) using the Florida probe with a standardized force of $0.25 \mathrm{~N}$... at least 16 sound natural teeth without crowns, bridgework or ill-fitting dental restorations"

Exclusion criteria: quote: "Exclusion criteria were: more than four pockets $\geq 5 \mathrm{~mm}$ - third molars and the distal sites of the second molars not included, caries with cavities, known previous hypersensitivity reactions to drugs, alcohol or drug addiction, severe liver or renal disease, psychiatric disorders, severe disability with multiple drug therapy, pregnancy or planned pregnancy and breast feeding. Neither should patients have been on treatment with antibiotics within the last 6 weeks prior to the prestudy visit, nor should they have treatment with anti-inflammatory or anti-cholinergic drugs"

Baseline gingivitis: not reported

Age at baseline (years): mean ages (SD): delmopinol 39 (8) years; CHX 40 (12) years; placebo 43 (11) years. Range not stated

Gender: males: $85.8 \%$, females: $14.2 \%$

Number randomised: 162 (CHX 50, delmopinol 53, placebo 53) (Note: discrepancy between the total number entering the study and the number included in per protocol analysis in each group at baseline)

Number evaluated: 133 (CHX 41, delmopinol 47, placebo 45)

\section{Comparison: $\mathrm{CHX}$ rinse versus delmopinol rinse* versus placebo rinse}

- CHX 0.2\% (Hibitane) ( $\mathrm{n}=50): 10 \mathrm{~mL}$, twice daily, 60 seconds, 6 months

- *Delmopinol (Decapinol) $(n=53): 10 \mathrm{~mL}$, twice daily, 60 seconds, 6 months. We excluded this arm from our risk of bias and analysis

- Placebo ( $\mathrm{n}=53)$ : $10 \mathrm{~mL}$, twice daily, 60 seconds, 6 months

Prophylaxis at baseline: quote: "After baseline, but before starting to rinse, the patients underwent professional cleaning of the teeth including supra- and subgingival scaling"

OHI: none. Quote: "..patients were instructed to brush their teeth as usual... No oral hygiene instructions were given before or during the study"

Partially supervised rinsing (supervised on weekdays, unsupervised at weekends) 
Lang 1998 (Continued)

Timing of mouthrinsing in relation to toothbrushing: if rinsing took place in connection with toothbrushing the rinsing should always be performed after the mechanical cleaning of the teeth

Outcomes

Gingivitis (gingival inflammation: GI, Löe 1963; BOP, bleeding yes/no Florida probe to bottom pocket, Löe 1967, Gibbs 1988); plaque (PI, Silness \& Löe 1964); calculus (Calculus Surface Index / Calculus Surface Severity Index (CSI/CSSI), Ennever 1961, Conroy 1968); stain (DI, Lang \& Räber 1981) assessed at baseline, 3 months and 6 months

Oral soft tissue examined and adverse effects reported during the study

Funding Not reported

Notes

Sample size calculation: quote: "The sample size calculation was based on BOP. The standard deviation was estimated to 14 and a difference of 10 percentage units between placebo and delmopinol was considered worth detecting. The calculation which was based on a power of $95 \%$ and a significance level of $\mathrm{P}=0.05$, resulted in a required number of 50 subjects in each treatment group"

Adverse effects: quote: "No serious adverse events were reported in this study... one of the most frequent adverse events when rinsing with delmopinol was a transient anaesthetic sensation in various parts of the oral mucosa. This event was reported as 'anaesthesia local' or 'paraesthesia'... Taste affection ('taste loss', 'taste perversion') was reported by $35 \%$ and $20 \%$ of the patients in the delmopinol group after 3 and 6 months, respectively...The patients in the chlorhexidine group.. reported this event to the same extent, namely $35 \%$ after 3 months and $14 \%$ after 6 months.. Staining of the teeth and tongue was reported more often in the chlorhexidine group than the delmopinol group both at the 3month ( $69 \%$ and $20 \%$ of patients in each respective group) and 6 -month examination ( $88 \%$ and $16 \%$ in each respective group)... The most common adverse events causing withdrawal from treatment by the 10 patients rinsing with chlorhexidine were, staining of teeth, especially black staining (four patients), altered or bad taste (four patients), burning tongue (three patients) and black or brown staining of the tongue (three patients). The patient rinsing with delmopinol who was withdrawn from treatment due to adverse events reported anaesthesia of mucous membrane, burning tongue, dry mouth and taste alteration. The withdrawal patient in the placebo group due to adverse events reported bad taste, stomach problems, and tendency to vomit"

Declarations/conflicts of interest: none declared, however, 2 of the authors are associated with Biosurface Pharma AB

\section{Risk of bias}

Bias Authors' judgement Support for judgement

Random sequence genera- Low risk tion (selection bias)

Quote: "Patients were allocated to treatment groups, according to a computer-generated randomization list, where treatment was balanced within blocks of six patients"

Comment: adequate method of random sequence generation

\begin{tabular}{|c|c|c|}
\hline $\begin{array}{l}\text { Allocation concealment } \\
\text { (selection bias) }\end{array}$ & Unclear risk & $\begin{array}{l}\text { Quote: "Packaging and labelling were carried out at an independent Clinical } \\
\text { Service Department in Uppsala, Sweden. The various test solutions were dis- } \\
\text { pensed in identical } 200 \mathrm{ml} \text { amber glass bottles" }\end{array}$ \\
\hline
\end{tabular}

Comment: it is not clear whether the assignment of subjects to groups was carried out at the independent Clinical Service Department

Blinding of participants and personnel (performance bias)

All outcomes
High risk

The authors report the study to be "double-blind"

Quote: "The various test solutions were dispensed in identical $200 \mathrm{ml}$ amber glass bottles." The placebo consisted "of the vehicle of active delmopinol solution except delmopinol and sodium hydroxide"

Quote: "..rinsing with chlorhexidine resulted in statistically significantly more staining than both placebo and delmopinol" 
Comment: while every effort was made to ensure blinding of participants and personnel, the higher level of staining of the tongue and teeth in the $\mathrm{CHX}$ group relative to the placebo group meant that participants could have worked out which group they were in and this could have affected their oral health behaviours and hence the outcomes. The direction of this potential bias is not clear

Blinding of outcome assessment (detection bias) All outcomes
High risk

The authors report the study to be "double-blind"

Quotes: "The various test solutions were dispensed in identical $200 \mathrm{ml}$ amber glass bottles" and "Assessments were performed by trained examiners....E ach examiner scored one type of parameter for all patients throughout the whole study" and "Rinsing with chlorhexidine resulted in statistically significantly more staining than both placebo and delmopinol" and "The decisions to exclude patients/visits were taken before breaking the blind"

Comment: while every effort was made to ensure blinding of outcome assessment, the higher level of staining of the tongue and teeth in the $\mathrm{CHX}$ group relative to the placebo group meant that outcome assessors could not be adequately blinded and this could have affected the outcome assessment. The direction of this potential bias is not clear
Incomplete outcome data High risk (attrition bias)

All outcomes
Different data are reported for the number of patients excluded from the per protocol analysis (Table 2) and the number of patients withdrawn from treatment and reasons for withdrawal (Table 3). After 6 months (Table 2) 17/103 $(16.5 \%)$ randomised participants were not included in the final analysis. Atrition by group (Table 2): CHX 9/50 (18\%), placebo 8/53 (15.1\%). Total number of patients withdrawn from treatment and reasons for withdrawals by group: CHX $(n=13,24 \%)$ adverse events $(n=10)$ and refusal to cooperate $(n=3)$; placebo $(n=2,4 \%)$, adverse events $(n=1)$, refusal to cooperate $(n=1)$

Comment: there are a high proportion of losses between the groups whichever source of information (Table 2/Table 3 ) is used and reasons for losses are unbalanced between the groups with a high proportion of the withdrawals in the $\mathrm{CHX}$ group due to adverse effects

All outcomes stated in the methods section are adequately reported in the results section

Information on means and SEs are presented graphically. From correspondence with the author, additional information was obtained on means and SEs which enabled calculation of SDs from SEs and inclusion of these data in the meta-analyses

Selective reporting (re- Low risk porting bias)
Although this is an experienced research team and the examiners were trained there is no mention of calibration or reliability of outcome measurements

Mean age appears similar across groups. $86 \%$ of participants were male but the breakdown for gender across groups is not reported

16 and 10 patients in the $\mathrm{CHX}$ and placebo groups were using medication at the time of the premedical screening $(P<0.001)$

Assessments of GI were performed directly after BOP. Quote: "thus the possible traumatising effect on the gingival crevice may have affected the results of the GI" 
Lopez-Jornet 2012

\section{Study characteristics}

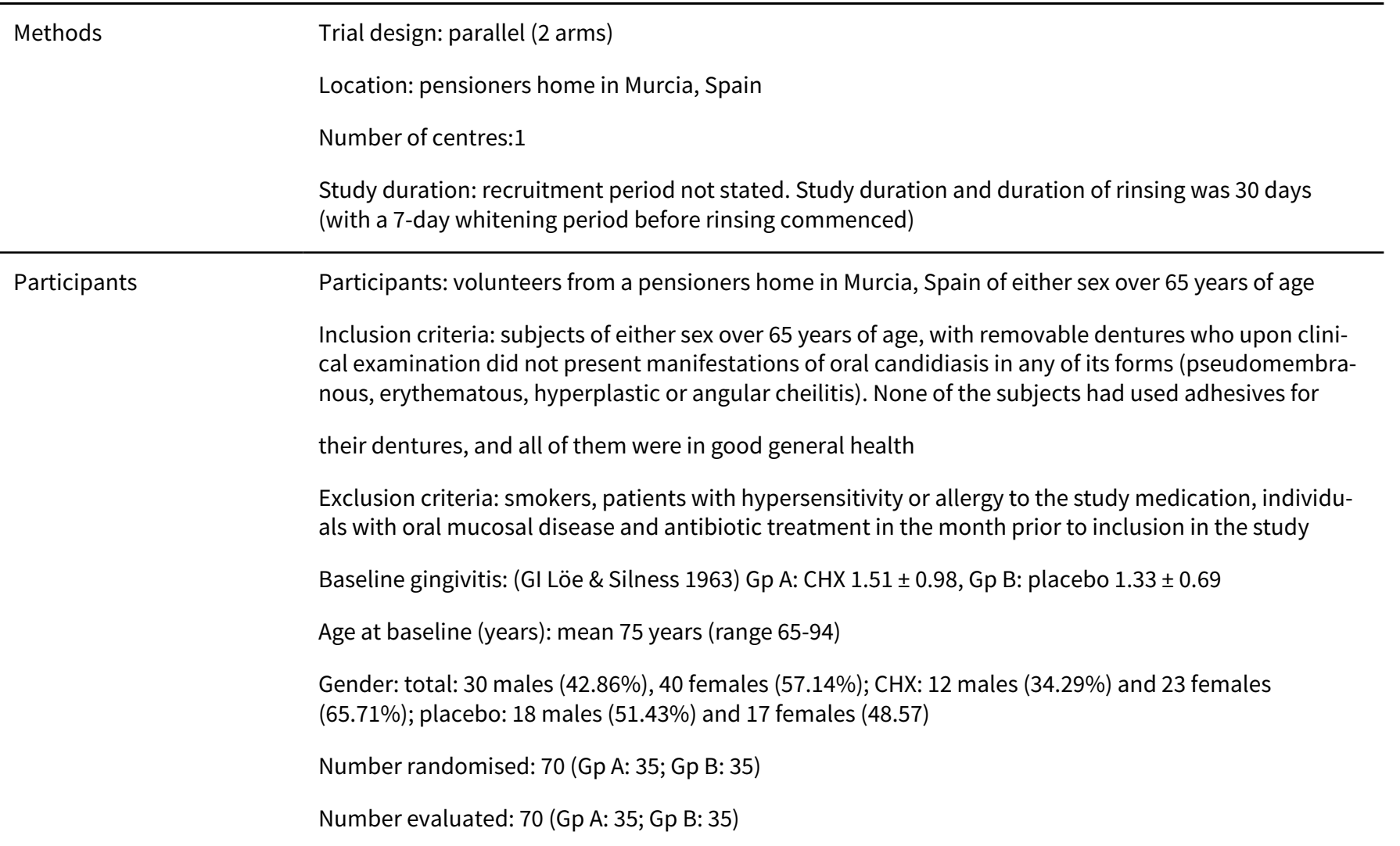

Interventions

\section{Comparison: $\mathrm{CHX}$ rinse versus placebo control rinse}

Gp A: CHX 0.2\%, 10 mL, 30 seconds, twice daily for 30 days

Gp B: placebo, same formulation without $\mathrm{CHX} 10 \mathrm{~mL}, 30$ seconds, twice daily for 30 days

Prophylaxis at baseline: none

$\mathrm{OHI}$ : both groups were supplied with a whitening rinse and toothbrush with $0.05 \%$ fluoridated toothpaste, and an instruction sheet and were advised to use for 7 days prior to recording of Silness \& Löe PI and $\mathrm{Gl}$. They also received instructions for accurate oral hygiene

Non-supervised rinsing

Timing of mouthrinsing in relation to toothbrushing: not reported

Postrinsing instructions: quote: "the subjects avoided rinsing with water, eating and drinking for 30 min following use of the mouthwash. They also received instructions for accurate oral hygiene, and were required to avoid all products other than those included in the study"

Outcomes

Gingivitis (ginginal inflammation: GI Löe \& Silness 1963) and plaque (Silness \& Löe 1964) measured after 30 days of rinsing

Microbiological evaluation of Candida albicans (colony forming units) measured at baseline and after 30 days of rinsing - not relevant to this review

Adverse effects: teeth/dentures/tongue staining, mucosal desquamation, alteration in taste sensation after 15 and 30 days of rinsing 
Lopez-Jornet 2012 (Continued)
Notes
Sample size calculation: not reported

Adverse effects: quote: "In reference to the presence of adverse effects, examination of the oral cavity in both groups revealed statistically significant differences after 15 days and at the end of the study (30 days) in terms of staining of the teeth/dentures and tongue. No patient showed mucosal desquamation or alterations in taste sensation"

Number and \% of participants with observed adverse effects at 30 days (Table 4): tongue staining: $\mathrm{CHX}$ : 11, 31.43\%; placebo 8, 22.86. Denture/dental staining: CHX 2, 5.71\%; placebo: 3, 8.57\%

Non-smokers

Declarations/conflicts of interest: not reported

The GI data appear to be transposed (Table 2 page 295). These were entered in the opposite order in RevMan for analysis

\section{Risk of bias}

Bias Authors' judgement Support for judgement

Random sequence genera- Unclear risk tion (selection bias)

Quotes: "randomized" and "the randomization list in the form of envelopes was generated using specifically designed software"

Comment: no description of the method of random sequence generation

\begin{tabular}{ll}
\hline $\begin{array}{l}\text { Allocation concealment } \\
\text { (selection bias) }\end{array}$ & $\begin{array}{l}\text { Quotes: "randomized" and "the randomization list in the form of envelopes } \\
\text { was generated using specifically designed software" and "both products were } \\
\text { supplied in identical opaque bottles" }\end{array}$ \\
& $\begin{array}{l}\text { Comment: mentioning the use of envelopes does not provide enough informa- } \\
\text { tion to make a judgement. It is not clear how the allocation sequence was con- } \\
\text { cealed before participants were randomised to groups }\end{array}$
\end{tabular}

\begin{tabular}{ll}
\hline $\begin{array}{l}\text { Blinding of participants } \\
\text { and personnel (perfor- }\end{array}$ & Quotes: "double-blind" and "both products were supplied in identical opaque \\
mance bias) & bottles" and "..both groups revealed statistically significant differences after \\
All outcomes & $\begin{array}{l}15 \text { days and at the end of the study (30 days) in terms of staining of the teeth/ } \\
\text { dentures and tongue." The placebo was the same formulation as the test rinse } \\
\text { without the CHX }\end{array}$
\end{tabular}

Comment: there does not appear to be significantly more staining in the $\mathrm{CHX}$ group compared to the placebo group

\begin{tabular}{|c|c|c|}
\hline $\begin{array}{l}\text { Blinding of outcome as- } \\
\text { sessment (detection bias) } \\
\text { All outcomes }\end{array}$ & Low risk & $\begin{array}{l}\text { Quotes: "double-blind" and "All examinations were carried out by a single ex- } \\
\text { perienced examiner.." and "..both groups revealed statistically significant dif- } \\
\text { ferences after } 15 \text { days and at the end of the study ( } 30 \text { days) in terms of staining } \\
\text { of the teeth/dentures and tongue" }\end{array}$ \\
\hline
\end{tabular}

Commet: there does not appear to be significantly more staining in the $\mathrm{CHX}$ group compared to the placebo group

\begin{tabular}{lll}
\hline $\begin{array}{l}\text { Incomplete outcome data } \\
\text { (attrition bias) } \\
\text { All outcomes }\end{array}$ & Low risk & There were no losses to follow-up \\
\hline $\begin{array}{l}\text { Selective reporting (re- } \\
\text { porting bias) }\end{array}$ & Low risk & All outcomes stated in the methods section are reported in the results section \\
\hline Other bias & Unclear risk & $\begin{array}{l}\text { The groups were comparable at baseline in relation to age, gender, gingivitis, } \\
\text { plaque and colony forming units of Candida albicans }\end{array}$
\end{tabular}




\section{Study characteristics}

\section{Methods}

Trial design: parallel (4 arms - 2 arms are relevant to this review)

Location: Faculty of Dentistry, National University of Nordeste, Corrientes, Argentina

Number of centres: 1

Study duration: the duration of recruitment is not stated. 2 studies are reported; the first was a 21-day study with participants abstaining from all oral hygiene practices, the second involved the use of $\mathrm{CHX}$ or placebo rinse as an adjunct to oral hygiene for 180 days. The second study is relevant to this review and is described here

Participants

Participants: dental students

Inclusion criteria: quote: "All had at least sixteen natural teeth including four molars"

Exclusion criteria: quote: "Patients affected with systemic diseases or on regular medication were excluded"

Baseline gingivitis: mean CHX: 0, mean placebo: 0

Age at baseline (years): mean: 20.9 years. Range not reported

Gender: male: $40 \%$, female: $60 \%$

Number randomised: 20 (Gp A: 10; Gp B: 10)

Number evaluated: not explicitly stated but appears to be 20 (Gp A: 10; Gp B: 10)

Interventions

\section{Comparison: $\mathrm{CHX}$ mouthrinse versus placebo mouthrinse}

$\mathrm{Gp} \mathrm{A}(\mathrm{n}=10)$ : $\mathrm{CHX}$ mouthrinse $(0.12 \%), 10 \mathrm{~mL}$, twice daily, 180 days

$\mathrm{Gp} \mathrm{B}(\mathrm{n}=10)$ : placebo mouthrinse, $10 \mathrm{~mL}$, twice daily, 180 days

The rinse duration was not reported

Prophylaxis at baseline: quote: "Clinically normal gingiva was obtained by scaling, polishing and twice daily tooth brushing"

OHI: quote: "Each subject participating in this study received regular instruction and reinforcement in personal plaque control procedures throughout the entire pre-experimental phase"

Assumed to be non-supervised rinsing

Timing of mouthrinsing in relation to toothbrushing: participants were advised to rinse $30 \mathrm{~min}$ after toothbrushing

Outcomes

Gingivitis (gingival inflammation: GI, Löe \& Silness 1967, severity (mean GI score of entire mouth) and occurrence (proportion of surfaces with GI $\geq 1$ ), gingival bleeding: GI, Löe \& Silness 1967 (proportion of surfaces with GI 2 or 3 )); plaque (PI, Greene \& Vermillion 1964); effect on oral tissues assessed at days 0 , 90 and 180 days 
Lucas 1999 (Continued)

\begin{tabular}{ll} 
Funding & Not reported \\
\hline Notes & Sample size calculation: not reported \\
& $\begin{array}{l}\text { Adverse effects: quotes: "The clinical and tactile evaluation of the oral tissues throughout the exper- } \\
\text { imental period did not reveal any type of irreversible pathology associated with the use of chlorhexi- } \\
\text { dine" and "some extrinsic staining was observed in the chlorhexidine group" } \\
\text { Non-smokers } \\
\text { Declarations/conflicts of interest: none stated. CHX and placebo were kindly supplied by the Microsules } \\
\text { Bernabo Laboratories, Buenos Aires, Argentina }\end{array}$
\end{tabular}

\section{Risk of bias}

\begin{tabular}{lll}
\hline Bias & Authors' judgement & Support for judgement \\
\hline $\begin{array}{l}\text { Random sequence genera- } \\
\text { tion (selection bias) }\end{array}$ & Unclear risk & $\begin{array}{l}\text { Quote: "The subjects were divided at random into four groups.." } \\
\text { Comment: no description of the method of random sequence generation }\end{array}$ \\
\hline $\begin{array}{l}\text { Allocation concealment } \\
\text { (selection bias) }\end{array}$ & Unclear risk & No information is provided \\
\hline $\begin{array}{l}\text { Blinding of participants } \\
\text { and personnel (perfor- } \\
\text { mance bias) }\end{array}$ & High risk & $\begin{array}{l}\text { Quote: "Neither the experimental subjects nor the examiner responsible for } \\
\text { the clinical examinations had previous knowledge of what group was rinsing } \\
\text { with chlorhexidine or placebo. (Chlorhexidine and placebo rinses were kind- } \\
\text { ly supplied... with a code number which was revealed after all data collection } \\
\text { was completed and tabulated.)" The composition of the placebo is not re- } \\
\text { ported. Although tooth staining and calculus were not measured the authors } \\
\text { note that "...some extrinsic tooth staining was observed in the chlorhexidine } \\
\text { group" and that "Supraginigival calculi were more numerous in the group us- } \\
\text { ing chlorhexidine.." }\end{array}$ \\
$\begin{array}{l}\text { Comment: the higher level of tooth staining and calculus in the CHX group } \\
\text { meant that participants could have worked out which group they were in and } \\
\text { this could have affected their oral health behaviours and hence the outcome. } \\
\text { The direction of this potential bias is unclear }\end{array}$
\end{tabular}

Blinding of outcome as-
sessment (detection bias)

All outcomes

Quote: "Neither the experimental subjects nor the examiner responsible for the clinical examinations had previous knowledge of what group was rinsing with chlorhexidine or placebo. (Chlorhexidine and placebo rinses were kindly supplied... with a code number which was revealed after all data collection was completed and tabulated)"

Although tooth staining and calculus were not measured the authors note that "...some extrinsic tooth staining was observed in the chlorhexidine group" and that "Supraginigival calculi were more numerous in the group using chlorhexidine.."

Comment: the higher level of staining in the $\mathrm{CHX}$ group meant that the outcome assessor could not be adequately blinded and this could have affected the outcome assessment. The direction of this potential bias is unclear

\begin{tabular}{lll}
$\begin{array}{l}\text { Incomplete outcome data } \\
\text { (attrition bias) } \\
\text { All outcomes }\end{array}$ & Low risk & $\begin{array}{l}\text { The number of participants at } 180 \text { days is not explicitly stated and the results } \\
\text { are presented by number of surfaces not number of participants. However it } \\
\text { would seem that all the participants were included in the data at } 180 \text { days }\end{array}$ \\
\hline $\begin{array}{l}\text { Selective reporting (re- } \\
\text { porting bias) }\end{array}$ & High risk & $\begin{array}{l}\text { Reported numbers represent number of surfaces of teeth evaluated rather } \\
\text { than the number of individuals. No measures of variance were reported for ei- } \\
\text { ther outcome. We estimated the SD for gingivitis (measured using the } \mathrm{Gl}) \text { as }\end{array}$ \\
\hline
\end{tabular}


the median of the SDs in the placebo/control groups from similar studies that also used the $\mathrm{Gl}$ at 6 months so that we could include the gingivitis data in the meta-analyses. Tooth staining is considered to be an important outcome but this is not adequately reported

Other bias Unclear risk

Training of the single examiner and intraexaminer reliability are not mentioned. The baseline demographics of the groups are not reported. There is no way to determine if the groups were similar at the start of the study

\section{Study characteristics}

Methods

Trial design: parallel, (3 arms - 2 arms relevant to this review)

Location: periodontal clinic, State University of Ponta Grossa, Brazil

Number of centres: 1

Study duration: recruitment period is not stated but the study duration and duration of rinsing was 42 days

Participants

Participants: males aged between 14 and 35 years

Inclusion criteria: minimum 16 permanent teeth (including 4 molars, but excluding teeth with excessive decay, crowns, bridges, fixed orthodontic appliances, and third molars)

Exclusion criteria: active periodontal disease; deposits of calculus; use of antibiotics, anti-inflammatory, and antibacterial or other drugs that could influence the study

Baseline gingivitis (completers): (GI, Löe \& Silness 1963) Gp A: mean 0.043 (SE 0.021); Gp B: mean 0.065 (SE 0.026); Gp C: mean 0.040 (SE 0.022)

Age at baseline (years): range 14 to 35 (not reported by group)

Gender: males only

Number randomised: not reported

Number evaluated: 31 (Gp A: 9; Gp B: 11; Gp C: 11)

Interventions

\section{Comparison: $\mathrm{CHX}$ digluconate rinse versus Plantago major rinse ${ }^{\star}$ versus placebo rinse}

$\mathrm{Gp} \mathrm{A}(\mathrm{n}=9$ analysed): $\mathrm{CHX}(0.12 \%), 10 \mathrm{~mL}$, once daily before bedtime

${ }^{\star} \mathrm{Gp} \mathrm{B}(\mathrm{n}=11$ analysed): same routine using Plantago major. We excluded this arm from our risk of bias and analysis

Gp B ( $\mathrm{n}=11$ analysed): same routine using placebo

Prophylaxis at baseline: participants had a thorough prophylaxis to remove plaque

OHI: yes. Quote: "Patients were subjected to a regular program of plaque control not supervised and instructed to swish $10 \mathrm{mls}$ of solution daily before bedtime"

Non-supervised rinsing

Timing of mouthrinsing in relation to toothbrushing: not reported

Postrinsing instructions: not reported 
Navarro 1998 (Continued)

Outcomes

Gingivitis (gingival inflammation: GI, Löe \& Silness 1963); plaque (PI, TQH, Turesky 1970); tooth staining (Lobene Stain Index 1968); adverse events (soft tissues), measured at 3 and 6 weeks follow-up

\begin{tabular}{ll}
\hline Funding & Not reported \\
\hline Notes & Sample size calculation: not reported \\
& Adverse effects: 1 person reported epithelial desquamation in the CHX group \\
& Declarations/conflicts of interest: unclear from translation \\
\hline
\end{tabular}

\section{Risk of bias}

\begin{tabular}{lll}
\hline Bias & Authors' judgement & Support for judgement \\
\hline $\begin{array}{ll}\text { Random sequence genera- } \\
\text { tion (selection bias) }\end{array}$ & Unclear risk & $\begin{array}{l}\text { Quote: "Patients were randomized into treatment group, positive control or } \\
\text { placebo" }\end{array}$ \\
& & Comment: no description of the method of random sequence generation \\
\hline
\end{tabular}

Allocation concealment $\quad$ Unclear risk No information is provided
(selection bias)

\section{Blinding of participants High risk} and personnel (performance bias)

All outcomes

Comment: the higher incidence of staining in the CHX group meant that par-
ticipants could have worked out which group they were in and this could have
affected their oral health behaviours and hence the outcome. The direction of
this potential bias is not clear

Comment: the higher incidence of staining in the $\mathrm{CHX}$ group meant that parthis potential bias is not clear
Quote: "Patients were randomised into treatment group, positive control or placebo following a double-blind scheme." A placebo solution containing distilled water, essence, colorant and methylparaben $0.1 \%$ was used. The CHX group showed an increase in tooth pigmentation at 6 months

Blinding of outcome as-
sessment (detection bias)

All outcomes
Quote: "Patients were randomised into treatment group, positive control or placebo following a double-blind scheme." No further information is given

Comment: the authors do not report precisely who was blinded, but even if this includes the outcomes assessors the higher level of staining in the active group meant that they may not have been adequately blinded and this could have affected the outcome assessment. The direction of this potential bias is not clear

\begin{tabular}{lll}
\hline $\begin{array}{l}\text { Incomplete outcome data } \\
\text { (attrition bias) } \\
\begin{array}{l}\text { All outcomes } \\
\text { Unclear risk }\end{array}\end{array}$ & $\begin{array}{l}\text { The number of patients at baseline is not reported. Results are presented for } \\
\text { the } 31 \text { subjects who completed the study. The authors report that } 1 \text { person in } \\
\text { the } \mathrm{CHX} \text { group left the study between } 0 \text { and } 21 \text { days due to epithelial desqua- } \\
\text { mation and that others withdrew from the study citing personal reasons } \\
\text { Comment: not enough information is provided }\end{array}$ \\
\hline $\begin{array}{l}\text { Selective reporting (re- } \\
\text { porting bias) }\end{array}$ & Low risk & $\begin{array}{l}\text { All outcomes stated in the methods section are adequately reported in the re- } \\
\text { sults section. We calculated SDs from the SEs reported so that the data could } \\
\text { be included in meta-analyses }\end{array}$
\end{tabular}

\begin{tabular}{ll}
\hline Other bias & Unclear risk \\
& $\begin{array}{l}\text { No information is provided on intra- and interexaminer reproducibility. At } \\
\text { baseline the groups had similar mean gingivitis, plaque and staining scores }\end{array}$ \\
\hline
\end{tabular}


Overholser 1990

\section{Study characteristics}

\begin{tabular}{|c|c|}
\hline \multirow[t]{3}{*}{ Methods } & $\begin{array}{l}\text { Trial design: parallel ( } 3 \text { arms - } 2 \text { arms are relevant to this review) } \\
\text { Location: assumed to be at the Baltimore College of Dental Surgery, Dental School, University of Mary- } \\
\text { land at Baltimore, USA }\end{array}$ \\
\hline & Number of centres: 1 \\
\hline & $\begin{array}{l}\text { Study duration: the recruitment period is not stated. Study duration and duration of rinsing was } 6 \\
\text { months }\end{array}$ \\
\hline \multirow[t]{8}{*}{ Participants } & Participants: healthy adults \\
\hline & $\begin{array}{l}\text { Inclusion criteria: quote: "...with pre-existing plaque and gingivitis but without clinical evidence of pe- } \\
\text { riodontitis... minimum of } 20 \text { sound natural teeth, and plaque and gingival index means } \geq 1.95 \text { as deter- } \\
\text { mined by the Turesky modification of the Quigley-Hein index (Turesky et al 1970) and the modified gin- } \\
\text { gival index (Lobene et al 1986), respectively" }\end{array}$ \\
\hline & $\begin{array}{l}\text { Exclusion criteria: quote: "Grossly carious, fully crowned, orthodontically banded, abutment and third } \\
\text { molar teeth were not included. Subjects with gross oral pathology or on antibiotic, antibacterial or an- } \\
\text { ti-inflammatory therapy were excluded from the study" }\end{array}$ \\
\hline & $\begin{array}{l}\text { Baseline gingivitis: (modified GI) Gp A: mean } 2.28 \text { (SE 0.03); Gp B: mean } 2.23 \text { (SE 0.02); Gp C: mean } 2.22 \\
\text { (SE 0.02) }\end{array}$ \\
\hline & $\begin{array}{l}\text { Age of completers (years): Gp A: mean 29.24; Gp B: mean 29.17; Gp C: mean 28.62. Range 21-62 years. } \\
\text { Gp A: range 23-62; Gp B: range 21-47; Gp C: range 22-42 }\end{array}$ \\
\hline & $\begin{array}{l}\text { Gender (completers): overall: male } 44 \text { (36\%), female } 80(65 \%) \text {. Gp A: male } 22(54 \%) \text {, female } 19(46 \%) \text {; Gp } \\
\text { B: male } 10(24 \%) \text {, female } 31(76 \%) \text {; Gp C: male } 12(29 \%) \text {, female } 30(71 \%)\end{array}$ \\
\hline & Number randomised: 128 (not reported by group) \\
\hline & Number evaluated: 124 (Gp A: 41; Gp B: 41; Gp C: 42) \\
\hline
\end{tabular}

\section{Comparison: $\mathrm{CHX}$ rinse versus Listerine rinse* versus control}

Gp A ( $\mathrm{n}$ completers $=41$ ): CHX (Peridex) 0.12\%,15 mL, twice daily, 30 seconds, 6 months

${ }^{*} \mathrm{Gp} \mathrm{B}$ ( $\mathrm{n}$ completers $=41$ ): Listerine, $20 \mathrm{~mL}$, twice daily, 30 seconds, 6 months. We excluded this arm from our risk of bias and analysis

Gp C ( $\mathrm{n}$ completers $=42)$ : control (flavoured coloured $5 \%$ hydroalcohol solution), $20 \mathrm{~mL}$, twice daily, 30 seconds, 6 months

Prophylaxis at baseline: quote: "Following the baseline examination, patients were given a complete prophylaxis to remove all plaque, calculus and extrinsic stain"

$\mathrm{OHI}$ : none given. Subjects followed their usual oral hygiene and dietary habits, but were instructed to refrain from using other mouthrinses

Rinsings were supervised twice daily on weekdays

Timing of mouthrinsing in relation to toothbrushing: not reported

Postrinsing instructions: quote: "Subjects were instructed not to rinse, eat or drink for 30 mins following use of the test rinses"

Outcomes

Gingivitis (gingival inflammation: modified GI, no reference; gingival bleeding: Interdental Bleeding Index, Caton \& Polson 1985); plaque (TQH, Turesky 1970 ); extrinsic tooth stain (Lobene Stain Index, Lobene 1968); supragingival calculus (Volpe 1965, Manhold 1965, Volpe 1967); assessed at 3 and 6 months follow-up. Plaque was collected for microbiological study (reported separately) 
Overholser 1990 (Continued)

Funding Q Quote: "This study was supported by a grant from the Warner-Lambert Company"

Notes Sample size calculation: not reported

Adverse effects: no abnormal soft tissue findings were noted in any group. Quote: "The PX group, showed significant increases $(P<0.001)$ in stain at 3 and 6 months, compared to its baseline"

Smokers: by group: Gp A: 5/41 (12\%); Gp B: 2/41 (5\%); Gp C: 6/42 (14\%)

Declarations/conflicts of interest: none stated

\section{Risk of bias}

\begin{tabular}{lll}
\hline Bias & Authors' judgement & Support for judgement \\
\hline $\begin{array}{l}\text { Random sequence genera- } \\
\text { tion (selection bias) }\end{array}$ & Low risk & $\begin{array}{l}\text { Quote: "Subjects were assigned to groups according to a computer-generated } \\
\text { random code" } \\
\text { Comment: adequate method of random sequence generation }\end{array}$ \\
\hline $\begin{array}{l}\text { Allocation concealment } \\
\text { (selection bias) }\end{array}$ & Low risk & $\begin{array}{l}\text { Product was dispensed in coded amber bottles. Product code was not dis- } \\
\text { closed to the examiners or recorded on case report forms }\end{array}$ \\
& $\begin{array}{l}\text { Comment: we consider it unlikely that participants and investigators enrolling } \\
\text { participants could foresee assignment }\end{array}$
\end{tabular}

Blinding of participants High risk and personnel (performance bias)

All outcomes
The authors state that the study is double-blind. The products were dispensed in coded amber bottles. The placebo control was a flavoured, coloured $5 \%$ hydroalcohol solution. Personnel dispensing rinses did not participate in any other aspect of the study. Quote: "Significantly greater $(P<0.01)$ extrinsic stain was observed in the PX group when compared to LA and the control at both 3 and 6 months.."

Comment: the higher level of tooth staining in the $\mathrm{CHX}$ group compared to the other 2 groups meant that participants could have worked out which group they were in and this could have affected their oral health behaviours and hence the outcome. The direction of this potential bias is not clear

Blinding of outcome as- High risk sessment (detection bias) All outcomes

\begin{abstract}
The authors state that the study is double-blind. The products were dispensed in coded amber bottles. Quotes: "Product code was not disclosed to the examiners or recorded on case report forms" and "Subjects refrained from all oral hygiene and use of experimental products on examination days until after the examination were completed in order to eliminate possible bias due to product odor" Separate forms were used to record the outcome data at each examination interval and the examiner did not have access to case report forms during the study. To assure maximum objectivity and reproducibility, 1 examiner was used for gingivitis, bleeding and plaque indices and another for the stain and calculus indices. Quote: "Significantly greater $(P<0.01)$ extrinsic stain was observed in the PX group when compared to LA and the control at both 3 and 6 months.."
\end{abstract}

Comment: despite the considerable efforts made to ensure blinding of outcome assessment, the higher level of staining in the $\mathrm{CHX}$ group meant that the outcome assessor could not be adequately blinded and this could have affected the outcome assessment. The direction of this potential bias is not clear
Incomplete outcome data Low risk (attrition bias)

All outcomes
Attrition at 6 months 4/128 (3.1\%). Losses not broken down by group. 3 subjects were lost because they were unable to comply with the supervised rinsing schedule. 1 subject in the Peridex group dropped out when stain began to form 
Overholser 1990 (Continued)

Comment: attrition is very low over the 6 months of the study

Selective reporting (re- Low risk porting bias)

All outcomes stated in the methods section were addressed in the results with the exception of microbiological plaque analysis which is reported in a separate paper. We calculated SDs from the SEs reported so that the data could be included in meta-analyses

Other bias Low risk

Efforts were made to standardise examiners in a series of sessions in which the examiners reviewed the clinical criteria in each index prior to the initiation of the study. There is no indication of inter- or intraexaminer reliability. To assure maximum objectivity and reproducibility, 1 examiner was used for gingivitis, bleeding and plaque indices and another for the stain and calculus indices

Comment: the standardisation sessions and use of 1 examiner to record each index would reduce inter- and intraexaminer variability.

Quote: "The treatment groups were well-balanced with respect to age and smoking status." The authors report a statistically significant imbalance in the distribution of sexes within groups. The treatment-by-sex interaction was investigated and was not significant. The groups appear balanced for gingival index, plaque scores, bleeding index, supragingival calculus and tooth staining at baseline (although no $P$ values for the differences are reported)

\section{Pereira 2011}

\section{Study characteristics}

Methods
Location: Department of Periodontics, Dental School, University of Fortaleza, Brazil
Number of centres: 1 centre
Study duration: recruitment period not stated. Study duration and duration of rinsing was 90 days
Participants: adult subjects from the University of Fortaleza
Inclusion criteria: quote: "Bleeding index > 20\%, presence of at least 20 natural teeth and absence of
supragingival calculus and other plaque retentive factors, such as carious cavity and restoration ex-
cess"
Exclusion criteria: quote: "Participants with medical disorders and under antimicrobial therapy, as well
as smokers, pregnant women and individuals presenting a probing depth > 3 mm were excluded from
the trial"
Baseline gingivitis: bleeding index, Gp A (0.27 \pm 0.02 ); Gp B (0.22 \pm 0.04 ); Gp C (0.23 \pm 0.07$)$
Age at baseline (years): mean age not reported. Age range 27 to 42 years (not reported by group)
Gender: male: 15 (50\%), female: 15 ( $50 \%)$ (not reported by group)
Number randomised: 30 (Gp A: $10 ;$ Gp B: 10 and Gp C: 10 )
Number evaluated: 30 (Gp A: $10 ; G p$ B: 10 and Gp C: 10 )

\section{Comparison: $\mathrm{CHX}$ rinse versus Ocimum gratissimum rinse ${ }^{\star}$ versus placebo control rinse}

Gp A $(n=10): C H X(0.12 \%) 10 \mathrm{~mL}, 1$ min, 3 times daily

${ }^{\star} \mathrm{Gp} \mathrm{B}(\mathrm{n}=10)$ : Ocimum gratissimum $(\mathrm{Og}) 10 \mathrm{~mL}, 1 \mathrm{~min}, 3$ times daily. We excluded this arm from our risk of bias and analysis 
Gp C ( $n=10)$ : triethanolamine (qsp), alcohol, water (qsp), nipagin (0.2\%), glycerine (2.5\%), aspartame (qsp); $10 \mathrm{~mL}, 1 \mathrm{~min}, 3$ times daily

Prophylaxis at baseline: quote: "After the initial examination all teeth of each subject were polished with a pumice and flossed to eliminate plaque remnants"

OHI: quote: "A personal 'kit' containing a new toothbrush, a commercial dentifrice with no anti-inflammatory properties and tests or control mouth rinses was given to all participants. They were instructed to brush their teeth for 1 minute, three times a day, using their habitual technique"

Non-supervised rinsing

Timing of mouthrinsing in relation to toothbrushing: quote: "After each brushing the participants rinsed with one of the formulations"

Postrinsing instructions: not reported

Duration of rinsing: 90 days

\begin{tabular}{ll}
\hline Outcomes & $\begin{array}{l}\text { Gingivitis (bleeding index (BOP), Ainamo \& Bay 1975); plaque (TQH, Turesky 1970); hard and soft oral } \\
\text { tissues for the presence of any adverse reaction. All at } 90 \text { days }\end{array}$ \\
\hline Funding & Quote: "Source of support - Nil" \\
\hline Notes & Sample size calculation: not reported \\
& $\begin{array}{l}\text { Adverse effects: quote: "The tests mouth rinses had a good acceptance and did not show formations of } \\
\text { abscess, ulceration or allergic reactions. However, three volunteers from CLX (CHX) group reported pig- } \\
\text { mentation and temporary taste disturbance" }\end{array}$ \\
& Non-smokers \\
Declarations/conflicts of interest: quote: "Conflict of interest: none declared"
\end{tabular}

\section{Risk of bias}

Bias Authors' judgement Support for judgement

Random sequence genera- Unclear risk $\quad$ The participants were assigned to groups by random permutations of 3
tion (selection bias)
Comment: no description of the method of random sequence generation

Allocation concealment $\quad$ Unclear risk No information is provided
(selection bias)

Blinding of participants High risk and personnel (performance bias)

All outcomes
Quotes: "double-blind" and "The bottles were previously coded to warrant that neither the examiner nor the participants knew their content, which was revealed by the pharmacist only after the study was completed" and ".. three volunteers from $\mathrm{CLX}(\mathrm{CHX})$ group reported pigmentation and temporary taste disturbance"

The control mouthrinse had the same formulation as the test rinses but without $\mathrm{CHX}$ or Og

Comment: the higher incidence of pigmentation and temporary taste disturbance in the CHX group meant that participants could have worked out which group they were in (or that they were in 1 of the active mouthrinse groups) and this could have affected their oral health behaviours

$\begin{array}{lll}\begin{array}{l}\text { Blinding of outcome as- } \\ \text { sessment (detection bias) }\end{array} & \text { High risk } & \begin{array}{l}\text { Quotes: "double-blind" and "The bottles were previously coded to warrant } \\ \text { that neither the examiner nor the participants knew their content, which was } \\ \text { All outcomes }\end{array} \\ \text { revealed by the pharmacist only after the study was completed" and ".. three }\end{array}$


Comment: the higher incidence of pigmentation in the $\mathrm{CHX}$ group meant that the outcome assessor could have worked out that these 3 participants were in the $\mathrm{CHX}$ group and could not be adequately blinded

Incomplete outcome data Low risk Quote: "All participants completed the trial"

(attrition bias)

Quote: "All participants completed the trial"

All outcomes

\section{Selective reporting (re- High risk} porting bias)
All outcomes stated in the methods section are adequately reported in the results section. Tooth staining is considered to be an important outcome but is not adequately reported

Quote: "At the beginning, there was no statistically significant difference between the control and tests groups with respect to PLI and $\mathrm{BI}(\mathrm{P}>0.05)$ means"

No information is provided on the balance of demographic factors (age, gender etc) between groups

Quote: "A single, previously calibrated examiner scored the BI and the plaque Index (PLI)." No information on intra- and inteexaminer reliability (from the previous calibration) are reported

\section{Study characteristics}

\begin{tabular}{|c|c|}
\hline \multirow[t]{4}{*}{ Methods } & Trial design: parallel ( 3 arms - 2 arms are relevant to this review) \\
\hline & Location: assumed university dental school, Iran \\
\hline & Number of centres: 1 \\
\hline & $\begin{array}{l}\text { Study duration: quote: "This study was conducted in } 2003 \text { for } 10 \text { months." Duration of rinsing and end } \\
\text { point for outcome assessment was } 6 \text { weeks }\end{array}$ \\
\hline \multirow[t]{8}{*}{ Participants } & $\begin{array}{l}\text { Participants: those attending for treatment in the periodontal clinic of the University of Mashhad, Den- } \\
\text { tal school, Iran }\end{array}$ \\
\hline & $\begin{array}{l}\text { Inclusion criteria: non-pregnant, no risk factors for increased susceptibility to bacterial endocarditis, } \\
\text { no current medications or antibiotics within past } 2 \text { months, moderate adult periodontitis, at least } 1 \text { site } \\
\text { with } 4 \mathrm{~mm} \text { or more pocket depth among their } 6 \text { lower anterior teeth and without a history of profes- } \\
\text { sional periodontal treatment within the past } 6 \text { months }\end{array}$ \\
\hline & Exclusion criteria: not reported \\
\hline & $\begin{array}{l}\text { Baseline gingivitis: (GI Löe \& Silness 1963) Gp A: mean } 1.97 \text { (SD 0.19); Gp B: mean } 1.78 \text { (SD 0.39); Group C } \\
\text { mean } 1.85 \text { (SD 0.14) }\end{array}$ \\
\hline & Age at baseline (years): mean age and age range are not reported \\
\hline & Gender: not reported \\
\hline & Number randomised: 30 (Gp A: 10; Gp B: 10; Gp C: 10) \\
\hline & Number evaluated: not reported \\
\hline
\end{tabular}


Rahmani 2006 (Continued)

Interventions

\section{Comparison: (ultrasonic SRP with $0.2 \% \mathrm{CHX}$ delivered through the tip of ultrasonically activated scaler) $^{\star}$ versus (CHX $0.2 \%$ + ultrasonic SRP with distilled water) versus (ultrasonic SRP using dis- tilled water alone)}

${ }^{\star} \mathrm{Gp} \mathrm{A}(\mathrm{n}=10)$ : ultrasonic SRP with $0.2 \% \mathrm{CHX}$ delivered through the tip of ultrasonically activated scale after baseline examination and 1 week later (data from this group were not extracted). We excluded this arm from our risk of bias and analysis

Gp B ( $n=10):$ CHX $(0.2 \%)+$ ultrasonic SRP with distilled water after baseline examination and 1 week later: $\mathrm{CHX}$ twice daily, 6 weeks

Gp C ( $n=10)$ : ultrasonic SRP using distilled water alone after baseline examination and 1 week later

Prophylaxis at baseline: quote: "Following the baseline measurements, the entire dentition was ultrasonically scaled and irrigated according to the treatment protocol for each group. After one week the same procedure was repeated for all groups until all supra- and subgingival calculus was removed according to both visual and tactile inspection"

OHI: quote: "At the first visit all patients were instructed tooth brushing using the Bass technique and interdental cleaning aids were given and given similar toothbrushes and toothpastes." OHI was reinforced 1 week after the baseline measurements and throughout the study

Non-supervised rinsing

Timing of mouthrinsing in relation to toothbrushing: not reported

Postrinsing instructions: not reported
Gingivitis (gingival inflammation: GI Löe \& Silness 1963, gingival bleeding: Gingival Bleeding Index, Ainamo \& Bay 1975); plaque (PI Silness \& Löe 1964) measured at 6 weeks

Probing pocket depth ( 6 sites on each tooth using a William's periodontal probe)

CAL ( 6 sites on each tooth using a William's periodontal probe) also measured at 6 weeks - not relevant to this review

Funding

Quote: "Research Vice-Chancellor of Mashhad University of Medical Sciences who supported the study by a grant"
Sample size calculation: not mentioned

Adverse effects: not reported

Declarations/conflicts of interest: not stated

\section{Risk of bias}

\begin{tabular}{lll}
\hline Bias & Authors' judgement & Support for judgement \\
\hline $\begin{array}{l}\text { Random sequence genera- } \\
\text { tion (selection bias) }\end{array}$ & Unclear risk & $\begin{array}{l}\text { Quote: "randomly divided into three treatment groups" } \\
\text { Comment: no description of the method of random sequence generation }\end{array}$ \\
\hline $\begin{array}{l}\text { Allocation concealment } \\
\text { (selection bias) }\end{array}$ & Unclear risk & No information is provided \\
\hline $\begin{array}{l}\text { Blinding of participants } \\
\text { and personnel (perfor- } \\
\text { mance bias) }\end{array}$ & High risk & $\begin{array}{l}\text { Comment: participants were not blinded. The different interventions meant } \\
\text { that participants would know which group they were in and this could have af- } \\
\text { fected their oral health behaviours and hence the outcome. The direction of } \\
\text { this potential bias is not clear }\end{array}$ \\
\hline
\end{tabular}


Rahmani 2006 (Continued)

Blinding of outcome as- Unclear risk Blinding of outcome assessment not mentioned but, even if there was outsessment (detection bias) come assessor blinding, it is likely that there would be more staining in the All outcomes $\mathrm{CHX}$ group thus compromising any blinding. However, tooth staining is not mentioned in the report so it remains unclear

$\begin{array}{ll}\begin{array}{l}\text { Incomplete outcome data } \\ \text { (attrition bias) }\end{array} & \text { Unclear risk } \\ \end{array}$

All outcomes weeks is not reported

Selective reporting (re- High risk
porting bias)
porting bias)
Adverse effects are not mentioned. Tooth staining is considered to be an important side effect of $\mathrm{CHX}$ mouthrinsing but this is not reported

\begin{tabular}{ll}
\hline Other bias & Unclear risk \\
$\begin{array}{l}\text { Quote: "There were no significant differences in the clinical parameters be- } \\
\text { tween test and control groups at the baseline examination" }\end{array}$ \\
\hline
\end{tabular}

Sanz 1989

\section{Study characteristics}

Methods

Trial design: parallel, 2 arms

Location: private practice limited to periodontics, Los Angeles, USA

Number of centres: 1

Study duration: recruitment period not stated. Study duration and duration of rinsing was 6 weeks

Participants

Participants: patients with periodontitis who required osseous periodontal surgery

Inclusion criteria: diagnosis of AAP Class III periodontitis; requiring periodontal osseous surgery; able to perform adequate mechanical plaque control

Exclusion criteria: patients receiving osseous grafts, pregnancy; diabetes or other metabolic diseases; receiving systemic antibiotics within 3 months of the start of the study; receiving anti-inflammatory or other systemic drugs capable of altering clinical response

Baseline gingivitis: not reported

Age at baseline (years): not reported

Gender: not stated

Number randomised: 40 (Gp A: 19; Gp B: 21)

Number evaluated: 38 (Gp A: 17; Gp B: 21)

Interventions

\section{Comparison: $\mathrm{CHXgluconate}$ rinse versus placebo rinse used postperiodontal surgery}

$\mathrm{Gp} \mathrm{A}(\mathrm{n}=19)$ : $\mathrm{CHX}(0.12 \%), 15 \mathrm{~mL}, 30$ seconds, twice daily

$\mathrm{Gp} \mathrm{B}(\mathrm{n}=21)$ : same routine using placebo

Prophylaxis at baseline: full-mouth SRP followed by osseous periodontal surgery in 1 quadrant (periodontal dressing then placed over entire surgical area). All participants received prescription for Tylenol II as pain relief

$\mathrm{OHI}$ : prior to surgery patients received instructions in oral hygiene 
Sanz 1989 (Continued)

Non-supervised rinsing

Timing of mouthrinsing in relation to toothbrushing: after usual oral hygiene practices

Postrinsing instructions: not reported

\section{Outcomes}

Gingivitis (gingival inflammation: modified Löe \& Silness GI 1963 using a pressure sensitive probe standardised at $30 \mathrm{~g}$ inserted to the bottom of the sulcus - reported as $\mathrm{Gl}$ severity (overall mean scores) and GI bleeding (scores 2 and 3), measured at 4 and 6 weeks (not measured at 1 and 2 weeks due to early healing of wound masking the inflammatory status of the gingiva))

Plaque (Silness \& Löe PI 1964 - reported as plaque accumulation and visible plaque), measured at 1, 2, 4 and 6 weeks

Extrinsic stain (stain/no stain scored from oral photographs of mandibular facial anterior teeth), measured at 6 weeks

Participants' subjective assessment (questionnaire on whether or not they liked the assigned rinse including taste and compliance), unclear when measured (reported at 1 and 2 weeks)

Periodontal status (pocket probing depth and CAL), measured at 4 and 6 weeks - not relevant to this review

Postsurgical soft tissue health (epithelialisation - poor/moderate/good), measured at 1, 2, 4 and 6 weeks - not relevant to this review

Pain (participants' own perception on 0 to 10 scale and by medication consumption pain on soft palpation with periodontal probe - none/mild/severe), measured at 1, 2, 4 and 6 weeks - not relevant to this review $\mathrm{OH}^{\prime \prime}$

Adverse effects: no case of desquamation was seen with the use of $0.12 \% \mathrm{CHX}$ rinse in this study. $24 \%$ of participants $(n=5)$ in the $\mathrm{CHX}$ group disliked the taste of the mouthrinse mainly because of a 'burning sensation' and 'a too strong taste'. Quote: "In the CHX group, $47.1 \%$ of the patients who did not have stain at the time of surgery developed light extrinsic dental stain" compared to $4.7 \%$ of patients in the placebo group

Declarations/conflicts of interest: not stated

\section{Risk of bias}

Bias Authors' judgement Support for judgement

Random sequence genera- Low risk tion (selection bias)
Quote: "they were randomly assigned to receive either chlorhexidine or placebo mouthrinse via a computer-generated random list"

Comment: adequate method of random sequence generation

\begin{tabular}{lll}
\hline $\begin{array}{l}\text { Allocation concealment } \\
\text { (selection bias) }\end{array}$ & Unclear risk information is provided
\end{tabular}

Blinding of participants High risk

and personnel (perfor-

The authors describe the study as double-blind. The control mouthrinse was

mance bias) identical to the test mouthrinse but without $\mathrm{CHx}$ gluconate. Quote: " ...at 6

All outcomes weeks, $47.1 \%(8 / 17)$ of the patients in the $\mathrm{CHX}$ group and $4.7 \%(1 / 21)$ in the placebo group showed light stain" (on the lower anterior teeth)

Comment: the higher level of tooth staining in the $\mathrm{CHX}$ group meant that participants could have worked out which group they were in and this could have 

this potential bias is not clear

\section{Blinding of outcome as- High risk} sessment (detection bias) All outcomes
The authors describe the study as double-blind. Clinical examinations were carried out. Quotes: "..by an independent clinical examiner (not the periodontist) at pre-surgery, 1, 2, 4, and 6 weeks post-surgically" and ".. at 6 weeks, $47.1 \%(8 / 17)$ of the patients in the CHX group and $4.7 \%(1 / 21)$ in the placebo group showed light stain" (on the lower anterior teeth)

Comment: the higher level of staining in the $\mathrm{CHX}$ group meant that the outcome assessor could not be adequately blinded and this could have affected the outcome assessment. The direction of this potential bias is not clear

\section{Incomplete outcome data Low risk} (attrition bias)

Attrition at 6 weeks overall 2/40 (5\%). Attrition by group: CHX 2/19 (11\%), all participants in the control group contributed data for the main outcomes of gingivitis, plaque and staining.

All outcomes
2 subjects were lost from the $\mathrm{CHX}$ group due to concomitant systemic antibiotic use. In the placebo group, 1 subject did not have a PD and PAL measurements taken and was excluded from analysis of these measurements

Comment: attrition is low (although all losses were from the CHX group) and do not appear to be related to the intervention
No measures of variance were reported. We estimated the SD for gingivitis (measured using the $\mathrm{GI}$ ) as the median of the SDs in the placebo/control groups from similar studies that also used the GI at 4-6 weeks so that we could include the gingivitis data in the meta-analyses

Other bias High risk

Training and calibration of the 1 (independent) clinical examiner is not mentioned. Baseline demographics of the subjects are not described. With the exception of visible plaque and stain, the baseline balance of the outcomes is not reported

Staining was poorly reported but appears that $94.2 \%$ of $\mathrm{CHX}$ group had staining at baseline compared to $33.3 \%$ in placebo

\section{Study characteristics}

\begin{tabular}{l} 
Methods \\
Location: unclear \\
Number of centres: 1 \\
Study duration: recruitment period not stated but the duration of the intervention was 6 months \\
\hline Participants: adults with a history of established gingivitis \\
Inclusion criteria: aged 18 to 65 years; minimum 16 natural teeth (including 4 anterior teeth in each \\
jaw); GI Löe \& Silness 1967 score greater than 0.7 \\
Exclusion criteria: evidence of periodontitis (probing pockets greater than 4 mm); medical history that \\
would interfere with participation; evidence of gross oral neglect \\
Baseline gingivitis: (GI Löe \& Silness 1967 ) Gp A: mean 1.57; Gp B: mean 1.53 \\
Age at baseline (years): Gp A: mean 33.5 (range 18 to 60 ); Gp B: mean 33.6 (range 18 to 65)
\end{tabular}


Number randomised: 139 (Gp A: 71; Gp B: 68) (208 including ineligible arm)

Number evaluated: 130 (Gp A: 66; Gp B: 64) (191 including ineligible arm)

Interventions

Comparison: (CHX digluconate rinse + gum care dentifrice) versus (placebo rinse + gum care dentifrice) versus (placebo rinse $+\mathrm{CHX}$ digluconate and zinc lactate dentifrice)*

*We excluded this arm from our risk of bias and analysis

Gp A $(n=71): C H X(0.12 \%)$ rinse + gum care dentifrice containing allantoin, urea, azulene, medical soap, calcium carbonate, and sodium monofluorophosphate 1100 ppm (Blend-a-med Parodontoseschutz), quantities and time not specified, twice daily

$\mathrm{Gp} \mathrm{B}(\mathrm{n}=68)$ : as above but the rinse was a placebo

Prophylaxis at baseline: quote: "After this examination all subjects received a dental prophylaxis in order to remove completely existing plaque and calculus"

$\mathrm{OHI}$ : new toothbrushes were available to all participants but no $\mathrm{OHI}$ was provided

Non-supervised rinsing

Timing of rinsing in relation to toothbrushing: immediately after toothbrushing

Postrinsing instructions: none

Duration of treatment: 6 months

Outcomes

Gingivitis: gingival inflammation (using a modification of GI Löe \& Silness 1967. An electronic periodontal pressure sensitive probe standardised at $25 \mathrm{~g}$ was used and mean scores were reported); gingival bleeding ( $\mathrm{Gl}=2$ was recorded when bleeding appeared on probing, measured at 3 and 6 months)

Plaque (PI Silness \& Löe 1964), measured at 3 and 6 months

Calculus (Volpe-Manhold Calculus Index (Volpe 1965)), measured at 3 and 6 months

Tooth staining (facial surfaces of the 12 anterior teeth. Comparisons made against a photographic standard; overall stain measured on a 0 to 6 scale; each tooth measured on a 0 to 4 scale for stain intensity; each tooth measured on a 0 to 6 scale for stain coverage), measured at 3 and 6 months

Oral soft tissue health attributable to product use, measured at 3 and 6 months

Funding Rinse and dentifrice supplied by Procter \& Gamble

Notes Sample size calculation: not reported

Adverse effects: quote: "No adverse effects on oral soft tissues were seen and no deviations from clinically normal were recorded which would indicate possible side effects which are related to any of the products used in this study." There was a statistically significant increase in calculus for the positive control group compared with the control group. Quote: "Statistically significant more overall staining, more intense staining and stain coverage per tooth were detected for the positive control group and the experimental group compared with the control group"

Declarations/conflicts of interest: nothing explicitly stated but 2 of the 5 authors were employees of Procter \& Gamble (manufacturers of the products being tested)

\section{Risk of bias}


Sanz 1994 (Continued)

Random sequence genera- Unclear risk tion (selection bias)
Quote: "Subjects were then stratified by plaque Index (PII) and gingival index (GI) and gender and they were randomly assigned to one of the three treatment groups"

Comment: no description of the method of random sequence generation

Allocation concealment Unclear risk No information is provided

(selection bias)

Blinding of participants and personnel (performance bias)

All outcomes
High risk

The study is described as double-blind. The control mouthrinse was Peridex without the CHX gluconate. Quotes: "All products were supplied in identical tubes and bottles, respectively" and "Statistically significant more overall staining, more intense staining and stain coverage per tooth were detected for the positive control group and the experimental group compared with the control group"

Comment: the higher level of staining in the $\mathrm{CHX}$ group than the placebo group meant that participants could have worked out which group they were in and this could have affected their oral health behaviours and hence the outcome. The direction of this potential bias is unclear

\section{Blinding of outcome as- High risk} sessment (detection bias) All outcomes
The study is described as double-blind. Quotes: "The oral examinations were carried out by 2 examiners each carrying out the same examinations throughout the study" and "Statistically significant more overall staining, more intense staining and stain coverage per tooth were detected for the positive control group and the experimental group compared with the control group"

Comment: the authors do not report precisely who was blinded, but even if this includes the outcomes assessors the higher level of staining in the $\mathrm{CHX}$ group meant that the outcome assessor could not be adequately blinded and this could have affected the outcome assessment. The direction of this potential bias is unclear
Incomplete outcome data Low risk (attrition bias)

All outcomes
Attrition at 6 months overall 17/208 (8.2\%). Attrition by group: $\mathrm{CHX}+$ gum care dentifrice 5/71 (7\%); placebo + gum care dentifrice 4/68 (6\%); placebo + experimental dentifrice $8 / 69(11.6 \%)$

Reason for losses: quote: "Reasons not associated with product use"

Comments: losses are low and although exact reasons for losses are not described and not broken down by group they are thought unlikely to have affected the outcome
The main outcome data presented in tables were broken down into subgroups with low and high initial gingivitis. The overall outcome data were presented only in graphs. No measures of variance were reported. We estimated the mean scores for gingivitis from the graphs. We estimated the SD for gingivitis (measured using the $\mathrm{GI}$ ) as the median of the SDs in the placebo/control groups from similar studies that also used the $\mathrm{Gl}$ at 6 months so that we could include the gingivitis data in the meta-analyses

Other bias Unclear risk

Training and calibration of the 2 examiners is not mentioned. Quote: "All treatment groups were well balanced at the beginning of the study with regard to age, gender, initial plaque and initial gingivitis index scores as summarized in table 1" 
Segreto 1986

\section{Study characteristics}

Methods Trial design: parallel, 3 arms

Location: University of Texas Health Science Centre, San Antonio, Texas, USA

Number of centres: 1

Study duration: recruitment period not stated. Study duration and duration of rinsing was 3 months

Participants

Participants: adult volunteers from the University of Texas Health Science Centre at San Antonio Texas and towns people from San Antonio

Inclusion criteria: minimum 16 natural teeth (including 4 molars)

Exclusion criteria: existing soft tissue damage caused by ill-fitting appliances; advanced periodontal disease; dental neglect needing prompt professional attention; unfavourable medical history; regular use of antibiotics; pregnancy

Baseline gingivitis (from Table 8): (GI Löe 1967) Gp A: mean 0.6745 (SD not reported); Gp B: mean 0.6468 (SD not reported); Gp C: mean 0.6750 (SD not reported)

Age at baseline (years) (from Table 7): Gp A: 31.29 (range 18 to 61); Gp B: mean 32.33 (range 18 to 60); Gp C: mean 30.74 (range 18 to 60 )

Gender: overall: male 234 (39\%), female 363 (61\%). Gp A: male 80 (40\%), female 122 (60\%); Gp B: male $81(41 \%)$, female 118 (59\%); Gp C: male $73(37 \%)$, female $123(63 \%)$

Number randomised: 597 (Gp A: 202; Gp B: 199; Gp C: 196)

Number evaluated: 454 (Gp A: 144; Gp B: 157; Gp C: 153)

Interventions

\section{Comparison: $0.2 \% \mathrm{CHX}$ gluconate rinse versus $0.12 \% \mathrm{CHX}$ gluconate rinse versus placebo rinse}

$\mathrm{Gp} \mathrm{A}(\mathrm{n}=202): \mathrm{CHX}(0.2 \%), 15 \mathrm{~mL}, 30$ seconds, twice daily

Gp B ( $\mathrm{n}=199)$ : CHX (0.12\%), $15 \mathrm{~mL}, 30$ seconds, twice daily

Gp C ( $=196)$ : same routine using placebo

Prophylaxis at baseline: after the baseline examination all subjects received a thorough prophylaxis

OHI: participants followed usual habits but were given fluoride toothpaste and soft toothbrush

Non-supervised rinsing

Timing of mouthrinsing in relation to toothbrushing: not reported

Postrinsing instructions: none reported

Outcomes

Gingivitis: gingival inflammation measured using GI (Löe 1967) and Papillary Marginal Gingivitis Index (PMGI) (de la Rosa \& Sturzenberger 197, visual inspection only - no probing); severity (mean extent of disease) and occurrence (meaning mean \% gingival sites with GI or PMGI score $\geq 1$ ). Gingival bleeding measured using GI (Löe 1967) mean \% of gingival sites with a GI score $\geq 2$ )

Plaque (plaque disclosed using standard red dye and quantity on facial and lingual surfaces assessed using TQH PI (Turesky 1970))

Oral soft tissue effects (visual-tactile examination of the oral mucosa), measured at 6 weeks and 3 months

All measured at 6 weeks and 3 months 
Segreto 1986 (Continued)

Funding
Quote: "The support of this study by the Procter \& Gamble Company, Cincinnati, Ohio, is gratefully acknowledged"
Notes

\section{Sample size calculation: not reported}

Adverse effects: quote: "With the exception of some subjective comments, there were no differences in the systematic examination of oral soft tissues which would indicate that adverse effects were produced by either chlorhexidine mouthrinse in comparison to the placebo mouthrinse." $12 \%$ of subjects in the $0.2 \%$ group dropped out because they did not like the mouthrinse, compared to $4 \%$ of the $0.12 \%$ group and $1 \%$ of the placebo subjects

The results are reported separately for examiner A and examiner B. Data for examiner A only were extracted and used in the analysis

Declarations/conflicts of interest: not stated

\section{Risk of bias}

Bias Authors' judgement Support for judgement

Random sequence genera- Unclear risk tion (selection bias)
Quote: "..they were stratified by age, sex, and gingivitis severity. Within strata they were assigned to one of three treatment groups by random permutations of 3"

Comment: no description of the method of random sequence generation

\begin{tabular}{lll}
\hline $\begin{array}{l}\text { Allocation concealment } \\
\text { (selection bias) }\end{array}$ & Unclear risk & No information is provided \\
\hline $\begin{array}{l}\text { Blinding of participants } \\
\text { and personnel (perfor- }\end{array}$ & Unclear risk & $\begin{array}{l}\text { The study is described as double-blind. Quotes: "Neither the subject nor the } \\
\text { dental examiners knew to which treatment the subject was assigned at any } \\
\text { All outcomes }\end{array}$ \\
$\begin{array}{l}\text { time during the study" and ".. the mouthrinses were supplied in pint bottles } \\
\text { with child-resistant closures." The placebo mouthrinse was identical in com- } \\
\text { position to the } 2 \text { active mouthrinses but without the CHX }\end{array}$
\end{tabular}

Comment: tooth staining is likely to have been noticeable in the $\mathrm{CHX}$ groups after 3 months, therefore participants could have worked out which group they were in and this could have affected their oral health behaviours and hence the outcome. However, tooth staining is not reported and there is not enough information upon which to base a judgement

Blinding of outcome as- Unclear risk sessment (detection bias) All outcomes
The study is described as double-blind. Quote: "Neither the subject nor the dental examiners knew to which treatment the subject was assigned at any time during the study"

Comment: tooth staining is likely to have been noticeable in the $\mathrm{CHX}$ groups after 3 months, and in this situation the outcome assessor could have worked out which individuals used $\mathrm{CHX}$ and not be adequately blinded. However, tooth staining is not reported and there is not enough information upon which to base a judgement

Incomplete outcome data High risk
(attrition bias)

All outcomes
Attrition at 3 months overall 143/597 (24\%) and by group: $\mathrm{CHX}(0.2 \%) 58 / 202$ (29\%), CHX (0.12\%) 42/199 (21\%), control 43/196 (22\%)

Reasons for losses: most were people who had moved or could not meet examination schedules, $12 \%$ of subjects in the $0.2 \%$ group dropped out because they did not like the mouthrinse, compared to $4 \%$ of the $0.12 \%$ group and $1 \%$ of the placebo subjects 
Segreto 1986 (Continued)

Selective reporting (reporting bias)

High risk

No measures of variance are reported so the data could not be included in meta-analyses. Furthermore, tooth staining is considered to be an important side effect of $\mathrm{CHX}$ mouthrinsing but this is not reported

Other bias Unclear risk
Training and calibration of the 2 examiners is not mentioned. We used data for examiner A only. Intraexaminer reliability is not reported for this examiner

Baseline demographic and clinical data for the 3 groups appear balanced

Southern 2006

\section{Study characteristics}

Trial design: parallel (3 arms - 2 arms are relevant to this review)
Location: Virginia, USA (type of setting not reported assumed to be a university dental school)
Number of centres: not reported
Study duration: recruitment period is not reported. Study duration and duration of rinsing was 3
months
months

Exclusion criteria: advanced periodontal disease (AAP IV or greater); use of antibiotics in the 90 days before the start of the study; need for antibiotics premedication; anterior facial restorations; use of a daily antigingivitis rinse in the 3 months before the start of the study; pregnancy; smoker; poor general health

Baseline gingivitis: not reported. Quote: "Randomization produced similar equivalent baseline groups that exhibited overall GI scores of 2 or 3"

Age at baseline (years): mean 25 (SD 7.67) (not reported by group but "Randomization produced similar equivalent baseline groups")

Gender: male $n=35(57 \%)$, female $n=28(43 \%)$ (not reported by group but "Randomization produced similar equivalent baseline groups")

Number randomised: 63 (not reported by group)

Number evaluated: 60 (not reported by group)*

*Text states: "final sample size of 60 (30 in each group)" - which does not make sense as there were 3 groups. Assumed final sample size 20 in each group ( 21 at baseline)

\section{Comparison: $\mathrm{CHX}$ rinse versus herbal rinse ${ }^{\star}$ versus placebo rinse}

$\mathrm{Gp} \mathrm{A}(\mathrm{n}=$ not reported, assumed 21$): \mathrm{CHX}(0.12 \%)$, half an ounce $(15 \mathrm{~mL})$, length of time not specified, twice daily (morning and evening)

${ }^{*} \mathrm{Gp} \mathrm{B}$ ( $\mathrm{n}=$ not reported, assumed 21): same as above with herbal rinse (filtered spring water, vegetable glycerin, echinacea, goldenseal, calendula, aloe, bloodroot, grapefruit seed extract, citric acid, spearmint oil, peppermint oil and cinnamon). This arm is not included in our risk of bias or analysis

$\mathrm{Gp} \mathrm{C}(\mathrm{n}=$ not reported, assumed 21$)$ : same as above with placebo rinse

Prophylaxis at baseline: none. Quote: "no prophylaxis was conducted prior to study initiation" 
OHI: all groups given same soft toothbrush and whitening fluoride toothpaste, and instructed to follow their usual oral hygiene routine (but not to use any other oral rinse during the study)

Partially supervised rinsing: as described in methods it appears to be non-supervised rinsing but the authors state that "A research assistant was responsible for product allocation and supervising rinsing procedures"

Timing of mouthrinsing in relation to toothbrushing: after usual mechanical oral hygiene routine Postrinsing instructions: not reported

Outcomes Gingivitis (gingival inflammation: GI Löe 1967 and BOP), measured at 1, 2 and 3 months

Plaque (PI Silness \& Löe 1964), measured at 1, 2 and 3 months

Oral soft tissue health, measured at baseline, 1, 2 and 3 months

\begin{tabular}{ll}
\hline Funding & Not reported \\
\hline Notes & Sample size calculation: not reported \\
& Adverse effects: not reported \\
& Non-smokers \\
& Declarations/conflicts of interest: not stated
\end{tabular}

\section{Risk of bias}

\begin{tabular}{lll}
\hline Bias & Authors' judgement & Support for judgement \\
\hline $\begin{array}{l}\text { Random sequence genera- } \\
\text { tion (selection bias) }\end{array}$ & Unclear risk & $\begin{array}{l}\text { Quote: "participants were randomized to one of three product groups" } \\
\text { Comment: no description of the method of random sequence generation }\end{array}$ \\
\hline $\begin{array}{l}\text { Allocation concealment } \\
\text { (selection bias) }\end{array}$ & Unclear risk & $\begin{array}{l}\text { Quotes: "participants were randomized to one of three product groups" and } \\
\text { "Individuals were identified by code numbers throughout the study" and "A re- } \\
\text { search assistant was responsible for product allocation and supervising rinsing } \\
\text { procedures" }\end{array}$
\end{tabular}

Comment: not enough information is provided

\begin{tabular}{|c|c|c|}
\hline $\begin{array}{l}\text { Blinding of participants } \\
\text { and personnel (perfor- } \\
\text { mance bias) } \\
\text { All outcomes }\end{array}$ & Unclear risk & $\begin{array}{l}\text { Quotes: "product identifiers were removed from all containers" and "The ex- } \\
\text { aminer and participants were blinded to product allocation" and "A research } \\
\text { assistant was responsible for product allocation and supervising rinsing pro- } \\
\text { cedures." It is not clear if the research assistant was blinded to allocation of } \\
\text { mouthrinses }\end{array}$ \\
\hline
\end{tabular}

Comment: the participants rinsed for 3 months. No information is provided about the ingredients in the placebo rinse. Tooth staining, although likely in the $\mathrm{CHX}$ group, is not mentioned in the report. The research assistant who was responsible for product allocation appears to have also supervised the mouthrinsing which may have compromised personnel blinding (although the methods imply that the rinsing was unsupervised so whether the rinsing was supervised/unsupervised is not clear)

Blinding of outcome as- Unclear risk sessment (detection bias) All outcomes
Quotes: "The examiner and participants were blinded to product allocation." Individuals were identified by code numbers throughout the study. Participants were given fluoride whitening toothpaste "to decrease the possible side effects of staining and lessen examiner bias" 
Comment: tooth staining is likely to have been noticeable in the $\mathrm{CHX}$ groups after 3 months, and in this situation the outcome assessor could have worked out which individuals used $\mathrm{CHX}$ and not be adequately blinded. However, tooth staining is not reported and there is not enough information upon which to base a judgement

Incomplete outcome data Low risk (attrition bias)

All outcomes
Attrition at 3 months overall 2/42 (4.8\%), 1 participant from each group. Reasons for loss to follow-up: personal reasons

Comment: attrition is low and there are no differential group losses

\section{Selective reporting (re- High risk} porting bias)
The GI was recorded but the mean GI was not reported. Instead the change in proportion of $\mathrm{Gl}$ scores of 2 or 3 compared to placebo was reported. No data are reported for probing pocket depth. Quote: "statistical analysis showed no statistically significant change in PPD in any of the groups." Tooth staining is considered to be an important side effect of $\mathrm{CHX}$ mouthrinsing but this is not reported

\begin{tabular}{ll}
\hline Other bias & Unclear risk \\
& Comment: no information provided on intraexaminer reproducibility
\end{tabular}

Quotes: "Randomization produced similar equivalent baseline groups" and "Randomization produced similar equivalent baseline groups that exhibited overall GI scores of 2 or 3"

Insufficient information is provided upon which to judge the baseline balance of demographic factors

\section{Study characteristics}

Methods
Location: USA, setting "clinical site"
Number of centres: 1

Study duration: the recruitment period is not reported but the duration of rinsing was 6 months

\section{Participants}

Participants: healthy adults

Inclusion criteria: at least 18 years of age with minimum 16 gradable natural teeth with 4 molars and in good general health. Subjects also had to have a minimum baseline whole mouth average $\mathrm{GI}$ score 0.50 , at least 10 bleeding sites and a maximum baseline plaque score of 3

Exclusion criteria: quote: "Patients were unable to continue in the study for the following reasons: participation in any other dental study; use of other oral care products; evidence of rampant caries, obvious periodontal disease, chronic neglect requiring urgent treatment, history of any medical diseases that may interfere with the study (e.g. bleeding tendencies, infectious diseases) wearing removable or fixed orthodontic devices; use of antibiotics or immunosuppressives within 1 week prior to baseline, 3- or 6-month exams; use of antiinflammatory drugs or analgesics within 48 hours of baseline, 3- or 6month exam; known hypersensitivity to chlorhexidine or tartrazine; oral prophylaxis outside of study; use of oral chlorhexidine products or participation in an oral rinse study within 3 months prior to baseline examination; pregnancy/nursing; noncompliance by missing more than five consecutive supervised rinses and/or more than $15 \%$ of all supervised rinses

Baseline gingivitis: (Löe \& Silness GI) Gp A: mean 0.794 (SE 0.025); Gp B: mean 0.792 (SE 0.018); Gp C mean 0.800 (SE 0.018); Gp D mean 0.814 (SE 0.020) 
Age at baseline (years): Gp A: mean 32.9; Gp B: mean 33.7; Gp C: mean 33.8; Gp D mean 34.3. Gp A: range 18-53; Gp B: range 18-59; Gp C: range 18-57; Gp D range 18-66

Gender: number in each group not reported. Male: Gp A: 60\%; Gp B: 63\%; Gp C: 59\%; Gp D: 62\%. Female: Gp A: 40\%; Gp B: 37\%; Gp C: 41\%; Gp D: 38\%

Number randomised: 366 (Gp A: 49; Gp B: 97; Gp C: 103; Gp D: 102)

Number evaluated (6 months): 298 (Gp A: 40; Gp B: 82; Gp C: 90, Gp D: 86)

\section{Interventions \\ Comparison: $\mathrm{CHX}$ rinse versus cetylpyridinium chloride rinse $(\mathrm{CPC} 0.075 \%)^{\text {a }}$ versus cetylpyridini- um chloride rinse (CPC $0.1 \%)^{b}$ versus placebo}

Gp A ( $n=49)$ : CHX (Peridex) 0.12\%: $15 \mathrm{~mL}$, twice daily, 30 seconds, 6 months

aGp B ( $n=97)$ : CPC 0.075\%: $15 \mathrm{~mL}$, twice daily, 30 seconds, 6 months. This arm was excluded from our risk of bias and analysis

bGp C ( $n=103)$ : CPC 0.10\%: $15 \mathrm{~mL}$, twice daily, 30 seconds, 6 months. This arm was excluded from our risk of bias and analysis

Gp D ( $\mathrm{n}=102)$ : placebo (of CPC): $15 \mathrm{~mL}$, twice daily, 30 seconds, 6 months

Prophylaxis at baseline: quote: "approximately 1 week following the baseline examination, qualifying subjects received a thorough dental prophylaxis"

$\mathrm{OHI}$ : supervised toothbrushing but unclear if there was $\mathrm{OHI}$

Quote: "From Monday to Friday, subjects came to the clinical site each morning for supervised brushing and rinsing.. Subjects were instructed to follow the same instructions in the evening and on the weekends"

Timimg of mouthrinsing in relation to toothbrushing: quote: "Subjects were instructed to brush with a $0.243 \%$ sodium fluoride toothpaste (Crest Cavity Protection) using a disposable Anchor toothbrush, rinse with water, then rinse with $15 \mathrm{ml}$ of product for 30 seconds"

Outcomes Gingivitis (GI Löe \& Silness 1963 to measure gingival inflammation and bleeding (GI scores 2 or 3));
plaque (TQH PI, Turesky 1970); oral soft tissue examinations; assessed at 3 and 6 months follow-up

Funding The test formulations (CPC) were provided by the Procter \& Gamble Company

Notes

Sample size calculation: not reported

Adverse effects: quote: "There were no serious adverse events reported during the study that were deemed related to the test products. OST examinations showed that subjects rinsing with the chlorhexidine treatment had significantly more "tongue lesion" comments at month 3 than those rinsing with either the CPC rinse or the placebo rinse. There were no significant differences between either of the CPC rinse groups and the placebo group in the number of subjects that had OST comments at 3 or 6 months"

Declarations/conflicts of interest: nothing explicit stated, however 2 of the co-authors (Dr Witt and Dr Gibb) were employees of the Procter \& Gamble Company at the time the study was published

\section{Risk of bias}

\section{Bias \\ Authors' judgement Support for judgement}

Random sequence genera- Unclear risk tion (selection bias)
Quotes: "Subjects were randomly assigned to... four treatments, balancing for gender and baseline mean GI score.." and "The randomization was performed such that the sample size per group ratio was 2:2:2:1 with 1 representing the chlorhexidine rinse group" 


Allocation concealment Unclear risk No information is provided

(selection bias)

\begin{tabular}{|c|c|c|}
\hline $\begin{array}{l}\text { Blinding of participants } \\
\text { and personnel (perfor- }\end{array}$ & Unclear risk & $\begin{array}{l}\text { Quotes: "double-blind" and "all mouthrinses were packed in identical amber } \\
\text { bottles." The control rinse was a placebo of CPC rinse. }\end{array}$ \\
\hline
\end{tabular}

Quote: "From Monday to Friday, subjects came to the clinical site each morn-

All outcomes ing for supervised brushing and rinsing.. Subjects were instructed to follow the same instructions in the evening and on the weekends"

Comment: it is not clear if the personnel supervised the rinsing were blinded to product allocation. Tooth staining is likely to have been noticeable in the CHX group after 6 months, therefore participants could have worked out which group they were in and this could have affected their oral health behaviours and hence the outcome. However, tooth staining is not reported and there is not enough information upon which to base a judgement

\section{Blinding of outcome as- Unclear risk} sessment (detection bias) All outcomes

\section{Quote: "double-blind"}

Comment: tooth staining is likely to have been noticeable in the $\mathrm{CHX}$ group after 6 months, and in this situation the outcome assessor could have worked out which individuals used $\mathrm{CHX}$ and not be adequately blinded. However, tooth staining is not reported and there is not enough information upon which to base a judgement
Incomplete outcome data High risk (attrition bias)

All outcomes
Attrition at 6 months all arms: 68/366 (18.6\%). Attrition by group: $\mathrm{CHX} 9 / 49$ (18\%), control 16/102 (15.7\%). Reasons for attrition: (not broken down by group) protocol violation (NSAIDS) $(n=15)$; medication use outside study protocol ( $n=41$ at 3 months, 17 at 6 months); not available ( $n=10$ at 3 months, 17 at 6 months); non-compliance ( $n=5$ at 3 months and 5 at 6 months); adverse event ( $n=5$ at 3 months and 8 at 6 months); non-study related medical reasons ( $n=2$ at 3 months and 6 at 6 months)

Comment: attrition was high and some losses were possibly related to the intervention

\begin{tabular}{lll}
\hline $\begin{array}{l}\text { Selective reporting (re- } \\
\text { porting bias) }\end{array}$ & High risk & $\begin{array}{l}\text { We calculated SDs from the SEs reported so that the data could be included in } \\
\text { meta-analyses. Tooth staining is considered to be an important side effect of } \\
\text { CHX mouthrinsing but this is not reported }\end{array}$ \\
\hline Other bias & Unclear risk & $\begin{array}{l}\text { Number of examiners not mentioned. Training and calibration of examiners } \\
\text { not mentioned }\end{array}$ \\
$\begin{array}{l}\text { Subjects balanced at baseline with regard to gender, age, gingival health and } \\
\text { plaque }\end{array}$
\end{tabular}

Taller 1993

\section{Study characteristics}

Methods $\quad$ Trial design: parallel (3 arms - 2 arms are relevant to this review)
Location: New Jersey, USA (type of setting not reported)
Number of centres: not reported


Taller 1993 (Continued)

Study duration: recruitment period is not reported. Study duration and duration of rinsing was 5 weeks

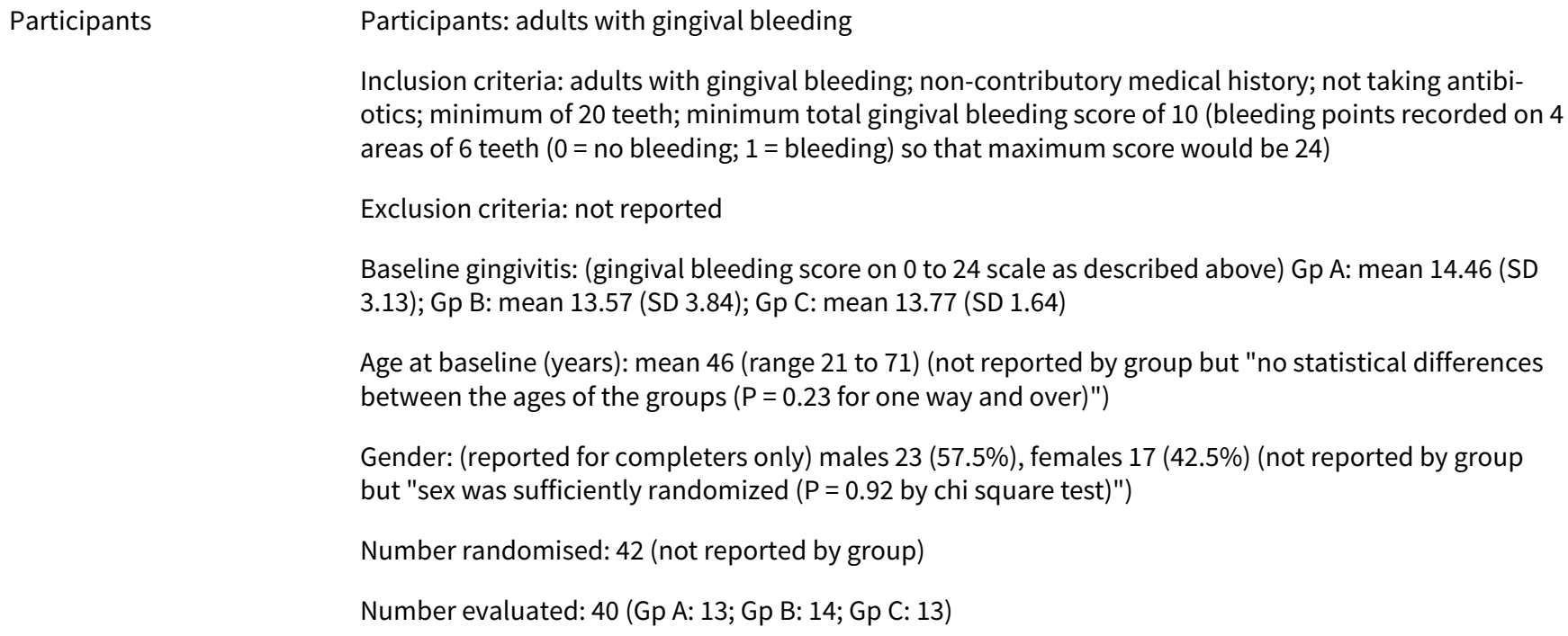

\section{Risk of bias}

\begin{tabular}{lll}
\hline Bias & Authors' judgement & Support for judgement \\
\hline $\begin{array}{l}\text { Random sequence genera- } \\
\text { tion (selection bias) }\end{array}$ & Unclear risk & Quote: "Subjects were randomly assigned to each group" \\
\hline
\end{tabular}


Taller 1993 (Continued)

Comment: no description of the method of random sequence generation

\begin{tabular}{lll}
\hline $\begin{array}{l}\text { Allocation concealment } \\
\text { (selection bias) }\end{array}$ & Unclear risk & No information is provided \\
\hline $\begin{array}{l}\text { Blinding of participants } \\
\begin{array}{l}\text { and personnel (perfor- } \\
\text { mance bias) }\end{array}\end{array}$ & High risk & $\begin{array}{l}\text { Blinding of participants is not stated and not possible with the interventions } \\
\text { being tested and this could have affected participants' oral health behaviours } \\
\text { all outcomes }\end{array}$
\end{tabular}

$\begin{array}{lll}\begin{array}{l}\text { Blinding of outcome as- } \\ \text { sessment (detection bias) }\end{array} & \text { Unclear risk } & \begin{array}{l}\text { Quote: "the same examiner scored all subjects and did not know the group } \\ \text { each subject was assigned to at any time" }\end{array}\end{array}$

All outcomes

Comment: blinding attempted but, as there is no information on tooth staining, it is not clear whether or not it would be possible for the outcome assessor to remain blind

$\begin{array}{ll}\begin{array}{l}\text { Incomplete outcome data } \\ \text { (attrition bias) }\end{array} & \text { Low risk } \\ \text { All outcomes } & \begin{array}{l}\text { Attrition at } 5 \text { weeks } 2 / 42(4.8 \%) \text {. Losses not broken down by group. Reason for } \\ \text { losses: did not return at } 5 \text { weeks }\end{array} \\ & \begin{array}{l}\text { Comment: it is not clear which groups the participants were lost from, howev- } \\ \text { er losses are low and are considered unlikely to have affected the outcome }\end{array}\end{array}$

\begin{tabular}{lll}
\hline $\begin{array}{l}\text { Selective reporting (re- } \\
\text { porting bias) }\end{array}$ & High risk & $\begin{array}{l}\text { Tooth staining is considered to be an important side effect of CHX mouthrins- } \\
\text { ing but this is not reported }\end{array}$ \\
\hline Other bias & Unclear risk & $\begin{array}{l}1 \text { examiner scored all subjects which eliminates interexaminer variability as a } \\
\text { source of bias. However, there is no mention of training or intraexaminer re- } \\
\text { producibility }\end{array}$
\end{tabular}

The groups are balanced at baseline for bleeding points, age and sex

\section{Study characteristics}

Methods
Location: Department of Periodontology, Ege University, Izmir, Turkey
Number of centres: 1
Study duration: quote: "subjects were recruited...over a period of 1 year between 2006 and $2007 . "$
Study duration and duration of rinsing was 4 weeks

Participants

Participants: adults with gingivitis attending the Department of Periodontology, Ege University

Inclusion criteria: 18 to 45 years old; gingivitis associated with dental plaque; no sign of destructive periodontal disease; clinical attachment loss less than $3 \mathrm{~mm}$; minimum 20 teeth (teeth that were grossly carious fully crowned or extensively restored, orthodontic banded, abutments, or third molars were not included in the tooth count)

Exclusion criteria: use of tobacco products; current or historic serious systemic disease affecting immune response (e.g. diabetes, immune disorders, hepatitis, HIV); use of antibiotics, anti-inflammatory, or immunosuppressive drugs within 3 months of the start of the study; periodontal therapy within 3 months of the start of the study; pregnancy or lactation; use of oral contraceptives

Baseline gingivitis: (Papillary Bleeding Index) Gp A: mean 1.47 (SD 0.5); Gp B: mean 1.44 (SD 0.7) 
Turkoglu 2009 (Continued)

Age at baseline (years): Gp A: mean 27.56 (SD 8.3) (range 18 to 45); Gp B: mean 25.44 (SD 5.6) (range 19 to 35 )

Gender: Gp A: males 13 (52\%), females 12 (48\%); Gp B: males 12 (48\%), females 13 (52\%)

Number randomised: 87 (Gp A: 45; Gp B: 42)

Number evaluated: 50 (Gp A: 25; Gp B: 25)

Interventions

\section{Comparison: $\mathrm{CHX}$ digluconate rinse versus placebo rinse}

$\mathrm{Gp} \mathrm{A}(\mathrm{n}=45)$ : $\mathrm{CHX}$ (concentration not reported), $10 \mathrm{~mL}, 1 \mathrm{~min}$, twice daily (morning and before bedtime)

$\mathrm{Gp} \mathrm{B}(\mathrm{n}=42)$ : same procedure as above using placebo rinse

Prophylaxis at baseline: not reported. Assumed none

OHI: all participants given the same fluoride toothpaste and toothbrush, and given toothbrush instruction (Modified Bass technique), instructed to brush twice daily

Non-supervised rinsing

Timing of mouthrinsing in relation to toothbrushing: $30 \mathrm{~min}$ after toothbrushing

Postrinsing instructions: no rinsing with water or eating or drinking for $30 \mathrm{~min}$ after using their allocated rinse

Outcomes

Gingivitis (gingival bleeding: Papillary Bleeding Index, Saxer 1975)

Plaque (PI, Quigley \& Hein 1962)

Calculus (scale not specified; references Oral Health of United States Adults. Epidemiology and oral disease prevention program. Bethesda, MD: National Institute of Dental Research; 1987; 159-67. NIH publication no 87-2868); probing depth* and gingival crevicular fluid cytokine levels*, all measured at 4 weeks using a Williams periodontal probe

Adverse effects, recorded weekly throughout the study

${ }^{\star}$ Not relevant to this review

Funding Quote: "This work was supported by an unrestricted grant from Procter \& Gamble. We would like to thank Drogsan Company for supplying the $\mathrm{CHX}$ and placebo mouthrinses"

Notes

Sample size calculation: based on detecting a 0.5 difference in plaque index and papillary bleeding index at $5 \%$ significance and $80 \%$ power, required 15 participants per group, which was achieved

Adverse effects: of the 25 who rinsed with CHX; 5 subjects experienced taste disturbances and 14 showed discolouration of teeth and/or tongue and 3 subjects had mucosal ulcerations

Non-smokers

Declarations/conflicts of interest: not stated

\section{Risk of bias}

\begin{tabular}{lll}
\hline Bias & Authors' judgement & Support for judgement \\
\hline $\begin{array}{l}\text { Random sequence genera- } \\
\text { tion (selection bias) }\end{array}$ & Unclear risk & $\begin{array}{l}\text { Quote: "patients were randomly assigned to CHX or placebo groups by taking } \\
\text { into account the gender, age and the extent of the gingivitis" }\end{array}$ \\
& & Comment: no description of the method of random sequence generation \\
\hline
\end{tabular}


Turkoglu 2009 (Continued)

Allocation concealment (selection bias)
Low risk

Quote: "patients were randomly assigned to $\mathrm{CHX}$ or placebo groups by taking into account the gender, age and the extent of the gingivitis by an independent periodontist who kept the allocation information confident until the data collection and biochemical analysis were completed"

Comment: we consider it unlikely that participants and investigators could foresee assignment of the intervention
Blinding of participants and personnel (performance bias)

All outcomes
High risk

Double-blind. Quotes: "The subjects .. were unaware of the type of mouthrinse provided to the patient" and "The placebo mouthrinse was composed of $\mathrm{CHX}$ mouthrinse ingredients except that it lacked the active ingredients (Chlorhexidine digluconate). Both $\mathrm{CHX}$ and placebo bottles were similar in appearance"

Comment: many of the participants in the $\mathrm{CHX}$ group experienced taste disturbances and/or discolouration of the teeth and/or tongue. Subjects in this group could have worked out which group they were in and this could have affected their oral health behaviours and hence the outcome. The direction of this potential bias is not clear

Blinding of outcome as-
sessment (detection bias)
All outcomes risk

Double-blind. Quote: "The same examiner recorded all clinical measurements over the course of the study that was unaware of the type of mouthrinse provided to the patient." In addition an independent periodontist performed the randomisation of participants to groups and kept the allocation information confidential until the analyses were completed

Comment: more than half of the subjects in the $\mathrm{CHX}$ showed discolouration of the teeth and/or tongue. It is unlikely that the clinical examiner could remain unaware of the group allocation of these subjects and this could have affected the outcome assessment. The direction of this potential bias is not clear

Incomplete outcome data High risk
(attrition bias)
All outcomes

Incomplete outcome data High risk

All outcomes
Losses to follow-up at 4 weeks 37/87 (42.5\%). By group: $\mathrm{CHX} \mathrm{20/45}$

(44.4\%),placebo 17/42 (40.5\%). Reasons for loss to follow-up: CHX: 3 discontinued the intervention and 17 were lost follow-up due to antibiotic usage, mucosal ulcerations, irregular mouthrinse usage. Placebo: 3 discontinued the intervention and 14 were lost follow-up due to antibiotic usage, mucosal ulcerations, irregular mouthrinse usage

Comment: the numbers lost in each group for each reason are not fully broken down. Losses are very high especially considering the short study duration. With such a high rate of attrition, if the missing participants in 1 group had a higher mean (e.g. gingivitis score) than those in the other group, as the attrition rate increased, so would the MD between groups

\begin{tabular}{lll}
\hline $\begin{array}{l}\text { Selective reporting (re- } \\
\text { porting bias) }\end{array}$ & Low risk & $\begin{array}{l}\text { All outcomes stated in the methods section are adequately reported in the re- } \\
\text { sults section. Adverse effects were only reported for the CHX group (presum- } \\
\text { ably there were no adverse effects in the control group) }\end{array}$ \\
\hline Other bias & $\begin{array}{l}\text { Quotes: "All measurements were performed by a single-blinded and calibrated } \\
\text { examiner.. the intra-examiner reliability was high as was revealed by intraclass } \\
\text { correlation coefficient } 0.87 \text { for PD measurements and } 0.85 \text { for plaque measure- } \\
\text { ments" and "There were no differences between CHX and placebo groups in } \\
\text { the distribution of the extent of gingivitis, gender and age. The groups were } \\
\text { balanced at baseline for PD, PBI, PI and Cl values }(\mathrm{P}>0.05) "\end{array}$
\end{tabular}

\section{Study characteristics}


Van Strydonck 2008 (Continued)

Methods Study design: parallel ( 3 arms 2 arms are relevant to this review)

Location: the Netherlands. Department of Periodontology, Academic Centre for Dentistry (ACTA)

Number of centres: 1

Study duration: Recruitment period is not reported. Study duration and duration of rinsing was 6 weeks

\section{Participants}

Participants: non-dental students at the University of Amsterdam

Inclusion criteria: quotes: "Good general health, no medical or dental history or medication which might interfere with the outcome or the progress of the study" and "a minimum of 18 scorable natural teeth excluding third molars or crowned teeth with porcelain or gold restorations. To be enrolled in the study, the subjects were required to have a minimum of $40 \%$ bleeding sites as determined by the Bleeding on Marginal Probing Index (BOMP)"

Exclusion criteria: quote: "Subjects were excluded if they had any physical limitations or restrictions which might preclude normal toothbrushing skills. They were also excluded if they had used an oral $\mathrm{CHX}$ product or had taken a systemic antibiotic or antiinflammatory drug for 3 consecutive days within the previous 3 months. Subjects with removable prostheses or orthodontic appliances were not allowed to participate"

Baseline gingivitis: Bleeding on Marginal Probing Index (Van der Weijden 1994) Gp A: 1.21 (SD 0.24), Grp B: 1.22 (SD 0.25), Gp C: 1.26 (SD 0.26)

Age at baseline: mean Gp A: 21, Gp B: 22, Gp C: 23. Age range 18-65 years

Gender: Gp A: male $21 \%$ female $79 \%$, Gp B: male $26 \%$ female $74 \%$, Gp C: male $37 \%$ female $63 \%$

Number randomised: 150 (Gp A 50, Gp B 50, Gp C 50)

Number evaluated: 140 (Gp A 47, Gp B 47, Gp C 46)

Interventions

\section{Comparison: (CHX mouthrinse and template control brush) versus (template control brush) ver- sus (template test brush (with slow-releasing delivery of $124 \mathrm{mg} \mathrm{CHX} \mathrm{digluconate))*}$}

Gp A ( $n=50)$ : template control brush and $\mathrm{CHX}$ rinse $0.2 \% \mathrm{CHX}$ digluconate: $10 \mathrm{~mL}$, twice daily, 60 seconds and brush twice daily for 1 min without dentifrice

Gp B ( $n=50)$ : template control brush: brush twice daily for 1 min without dentifrice

${ }^{\star} \mathrm{GPC}(\mathrm{n}=50)$ : test brush (with slow-releasing delivery of $124 \mathrm{mg} \mathrm{CHX}$ digluconate): brush twice daily for

$1 \mathrm{~min}$ without dentifrice. We excluded this arm from our risk of bias and analysis

Prophylaxis at baseline: quote: "At baseline subjects received a supragingival prophylaxis to render them plaque and stain free"

OHI: quote "..instructed to brush twice daily without a dentifrice for $1 \mathrm{~min}$ (in the morning and in the evening)"

Non-supervised rinsing

Timing of mouthrinsing in relation to toothbrushing: rinsed twice daily after toothbrushing without a dentifrice

Postrinsing instructions: quote: "subjects were asked to refrain from rinsing eating or drinking for 30 mins after using their assigned product"

Outcomes

Gingivitis (gingival bleeding: Bleeding on Marginal Probing Index (Saxton \& Van der Ouderaa, Van der Weijden 1994); plaque (Silness \& Löe 1964, Danser 2003); stain (Gründermann Modified Stain Index (Gründermann 2000) all measured at 3 and 6 weeks 
Van Strydonck 2008 (Continued)

Funding Quote: "This study was supported by a grant from Oral-B Laboratories, Belmont, CA, USA." GSK provided the Corsodyl mouthrinse

Notes

No dentifrice was used when toothbrushing

Sample size calculation: very detailed description provided. Quote: ".... a sample size of 45 subjects per treatment group was needed to ensure an $80 \%($ power $=1-\beta)$ or greater chance of detecting differences of $\geq 0.11$ whole-mouth BOMP units"

Adverse effects: apart from tooth staining, quotes: "no adverse events were reported" and "No differences were detected in the proportion of oral tissue abnormalities among the groups, with the exception of the tongue. Changes noted were the presence of stain or discoloration on the tongue. Treament $\mathrm{Ctb}+\mathrm{R}(\mathrm{Gp} A)$ yielded a statistically significantly $(\mathrm{P}=0.0001)$ greater proportion of abnormal observations than treatments Ttb (Gp C, excluded from this review) and Ctb (Gp B)"

Declarations/conflicts if interest: quote: "The authors declare that they have no conflict of interests"

\section{Risk of bias}

\section{Bias Authors' judgement Support for judgement}

Random sequence genera- Low risk tion (selection bias)

Quote: "Randomisation was performed using a computer-generated list of random-numbers"

Comment: adequate method of random sequence generation

\begin{tabular}{|c|c|c|}
\hline $\begin{array}{l}\text { Allocation concealment } \\
\text { (selection bias) }\end{array}$ & Unclear risk & $\begin{array}{l}\text { The study co-ordinator was responsible for allocation concealment } \\
\text { Comment: not enough information is provided }\end{array}$ \\
\hline
\end{tabular}

$\begin{array}{ll}\begin{array}{l}\text { Blinding of participants } \\ \text { and personnel (perfor- }\end{array} & \text { High risk } \\ \begin{array}{l}\text { mance bias) } \\ \text { All outcomes }\end{array} & \begin{array}{l}\text { Blinding of participants is not possible due to the interventions being tested: } \\ \text { this could have affected participants' oral health behaviours and hence the } \\ \text { outcome. The direction of this potential bias is unclear }\end{array}\end{array}$

Blinding of outcome as- High risk sessment (detection bias) All outcomes
Quote: "Study products were coded and distributed to the subjects in a location away from the examiners to ensure and maintain blinding.... the examiners were blind to treatment randomization and records of earlier examinations were not available at the time of re-examinations... One examiner assessed all plaque scores and performed all stain evaluations... Another examiner assessed all the bleeding scores using BOMP and all safety evaluations"

Quote: "The mean stain scores for treatment $\mathrm{Ctb}+\mathrm{R}(\mathrm{CHX})$ were statistically greater $(\mathrm{P}=0.0001)$ than for treatments $\mathrm{Ttb}(\mathrm{CHX}$ template toothbrush) and $\mathrm{Ctb}$ (control toothbrush)"

Comment: despite the efforts made to ensure blinding of outcome assessment, the higher level of tooth staining in the $\mathrm{CHX}$ rinse group meant that examining clinicians could not be adequately blinded and this could have affected the outcome assessment. The direction of this potential bias is unclear

Incomplete outcome data Low risk (attrition bias)

All outcomes
Attrition: all arms: $10 / 150$ (6.7\%). By group: $\mathrm{CHX}$ rinse $3 / 50$ (6.0\%), template control brush 3/50 (6\%). Reasons for losses not broken down by group. Subjects lost after randomisation and before baseline examination: 4 were disqualified, 4 refused to participate and 2 were excluded for other reasons

Comment: all losses were after randomisation before the start of the trial. They were balanced across groups 
Van Strydonck 2008 (Continued)

Selective reporting (re- Low risk All outcomes stated in the methods sections were reported in the results secporting bias) tion

Other bias

Low risk
Quote: "Both examiners were well-trained and had been involved in previous studies." Calibration is not mentioned

There was no statistically significant difference in mean age, or whole mouth plaque and gingival bleeding levels

\section{Weitz 1992}

\section{Study characteristics}

\begin{tabular}{l} 
Methods \\
Locaton: geriatric facility, USA \\
Number of centres: 1 \\
Study duration: recruitment period not stated. Study duration and duration of rinsing was 60 days \\
\hline Participants: residents in a geriatric facility \\
Inclusion criteria: residents in the geriatric facility receiving "emergency only" care. Quote: ".. at least 60 \\
years old and in good health" \\
Exclusion criteria: quote: "Patients presenting with a history of antibiotic therapy within the last 3 \\
months were not accepted into the study sample" \\
Baseline gingivitis: GI (Löe \& Silness 1963 ) Gp A: 1.88, Gp B: 1.80. SD not reported \\
Age at baseline: at least 60 years old at recruitment, mean 83 years. Not reported by group \\
Gender: overall: male 12 (33.33\%), females 24 (66.66\%). Not reported by group \\
Number randomised: 36 (number in each group is not reported). 12 with removable dentures, 12 with \\
overdentures and 12 with no prosthesis \\
Number evaluated: 36 (number in each group is not reported)
\end{tabular}

\section{Interventions Comparison: $\mathrm{CHX}$ rinse versus placebo rinse}

Gp A: CHX (0.12\%) ( $\mathrm{n}=$ not reported): $15 \mathrm{~mL}$, twice daily, 30 seconds

Gp B: placebo ( $\mathrm{n}=$ not reported): $15 \mathrm{~mL}$, twice daily, 30 seconds

Prophylaxis at baseline: none, prophylaxis performed after final measurements at 60 days

OHI: none. Quote: ".. instructed to continue their normal oral hygiene routine"

Non-supervised rinsing

Timing of mouthrinsing in relation to toothbrushing: not reported

Postrinsing instructions: not reported

Outcomes

Gingivitis (gingival inflammation: GI (Löe \& Silness 1963), measured at 60 days

Plaque (PI Silness \& Löe 1964), measured at 60 days 
Weitz 1992 (Continued)

\begin{tabular}{ll} 
Funding & Not reported \\
\hline Notes & Sample size calculation: not reported \\
& Adverse effects: not reported \\
& Declarations/conflicts of interest: not reported
\end{tabular}

\section{Risk of bias}

\begin{tabular}{lll}
\hline Bias & Authors' judgement & Support for judgement \\
\hline $\begin{array}{l}\text { Random sequence genera- } \\
\text { tion (selection bias) }\end{array}$ & Unclear risk & Quote: "patients were randomly divided into two groups" \\
& & Comment: no description of the method of random sequence generation \\
\hline $\begin{array}{l}\text { Allocation concealment } \\
\text { (selection bias) }\end{array}$ & Unclear risk & No information is provided \\
\hline $\begin{array}{l}\text { Blinding of participants } \\
\text { and personnel (perfor- } \\
\text { mance bias) } \\
\text { All outcomes }\end{array}$ & Unclear risk & $\begin{array}{l}\text { The study is not described as a single-blind study but the control group rinsed } \\
\text { with a quote "matched placebo". Information on tooth staining is not reported }\end{array}$ \\
\hline
\end{tabular}

\begin{tabular}{|c|c|c|}
\hline $\begin{array}{l}\text { Blinding of outcome as- } \\
\text { sessment (detection bias) } \\
\text { All outcomes }\end{array}$ & Unclear risk & $\begin{array}{l}\text { Blinding of outcome assessor is not mentioned and is unlikely. Information on } \\
\text { tooth staining is not reported }\end{array}$ \\
\hline
\end{tabular}

\begin{tabular}{|c|c|c|}
\hline $\begin{array}{l}\text { Incomplete outcome data } \\
\text { (attrition bias) } \\
\text { All outcomes }\end{array}$ & Low risk & There were no losses to follow-up \\
\hline $\begin{array}{l}\text { Selective reporting (re- } \\
\text { porting bias) }\end{array}$ & High risk & $\begin{array}{l}\text { No measure of variance was reported. We were unable to estimate the SD and } \\
\text { therefore could not include these data in meta-analyses. Furthermore, tooth } \\
\text { staining is considered to be an important side effect of CHX mouthrinsing but } \\
\text { this is not reported. Adverse effects are not reported }\end{array}$ \\
\hline \multirow[t]{2}{*}{ Other bias } & Unclear risk & $\begin{array}{l}\text { No information is provided on number of examiners and examiner training } \\
\text { and calibration }\end{array}$ \\
\hline & & $\begin{array}{l}\text { Quote: "At baseline, the active and control groups were not statistically differ- } \\
\text { ent (independent t-test, } P<0.05 \text { ) with respect to age, gingival index or plaque } \\
\text { index" }\end{array}$ \\
\hline
\end{tabular}

Zimmer 2006

\section{Study characteristics}

Trial design: parallel (4 arms - 2 arms relevant to this review)
Location: Department of Operative and Preventive Dentistry and Endodontics, Heinrich Heine Universi-
ty of Düsseldorf, Germany
Number of centres: 1
Study duration: recruitment period is not stated. Study duration and duration of rinsing was 8 weeks


Zimmer 2006 (Continued)

Participants
Participants: adult blood donors who visited the blood bank of the University Hospital, Düsseldorf, Germany in July and August 2004

Inclusion criteria: minimum Papillary Bleeding Index and Modified Proximal Plaque Index scores per tooth of 0.5 and 1.5 respectively

Exclusion criteria: wearing fixed orthodontic appliances; severe periodontitis (defined as CAL $5 \mathrm{~mm}$ or greater in a minimum of 3 teeth); long-term use of drugs with anti-inflammatory effects within 1 month prior and/or during the study; removable dentures; less than 20 natural teeth; regular use of dental floss or antimicrobial mouthrinses during past 3 months; furcation involvement; pathological tooth mobility

Baseline gingivitis: (Papillary Bleeding Index) Gp A: mean 1.23 (SD 0.44); Gp B: mean 1.25 (SD 0.45); Gp C: mean 1.19 (SD 0.44); Gp D: mean 1.27 (SD 0.45)

Age at baseline (years): mean 31.7 (range 20 to 64.4) (not reported by group but authors state that there were no statistically significant differences between groups)

Gender: 78 (50\%) males, 78 (50\%) females (not reported by group)

Number randomised: 156 (Gp A: 39; Gp B: 39; Gp C: 39; Gp D: 39)

Number evaluated: 156 (Gp A: 39; Gp B: 39; Gp C: 39; Gp D: 39)
Interventions

\section{Comparison: ( $\mathrm{CHX}+$ sodium fluoride ( $\mathrm{NaF}$ ) rinse and toothbrushing) versus (cetylpyridinium chlo- ride (CPC) + sodium fluoride (NaF) rinse and toothbrushing) a versus (flossing and toothbrushing) versus toothbrushing}

Gp A ( $n=39): \mathrm{CHX}(0.06 \%)+0.025 \% \mathrm{NaF}$, amount not specified, 30 seconds, once daily (after toothbrushing at bedtime)

${ }^{*} \mathrm{Gp} \mathrm{B}(\mathrm{n}=39): \mathrm{CPC}(0.1 \%)+0.025 \% \mathrm{NaF}$, amount not specified, 30 seconds, once daily (after toothbrushing at bedtime). We excluded this arm from our risk of bias and analysis

${ }^{\star} \mathrm{Gp} \mathrm{C}(\mathrm{n}=39)$ : flossing once daily (after toothbrushing at bedtime). We excluded this arm from our risk of bias and analysis

$\operatorname{Gp} D(n=39)$ : usual toothbrushing routine

Prophylaxis at baseline: quote: "The screening examination was followed by calculus removal in the lower front teeth"

OHI: quote: "Participants received brief instructions for the procedure they had to perform in addition to using the toothbrush, dental floss and oral rinsing. The dental floss instruction was demonstrated with a plastic tooth model did not exceed 2 minutes. The subjects were advised to brush their teeth in the usual manner. No instructions concerning brushing technique and brushing time were given." All participants received same toothbrush and fluoride toothpaste

Non-supervised rinsing

Timing of mouthrinsing in relation to toothbrushing: after brushing with toothpaste

Postrinsing instructions: not reported
Gingivitis (gingival bleeding: Papillary Bleeding Index, Saxer \& Muhlemann 1975); plaque (Modified Proximal Plaque Index, Lange 1977 and Zimmer 2005 and Quigley and Hein Index, Quigley and Hein 1962); adverse effects ("discomfort in taste, discomfort in sensibility, gingival damage, gingival bleeding, staining of teeth and tongue, mouth burning during application, and white plaque on tongue immediately after use"), all measured at 4 and 8 weeks

Funding Quote: "This study was supported by GlaxoSmithKline"

Notes Sample size calculation: not reported


Adverse effects: reported with no double counting - if any participant experienced more than 1 adverse effect, only the most 'relevant' one was reported

- At 4 weeks: no side effects: Gp A: 21/39, Gp B: 15/39, Gp C: 36/39, Gp D: 37/39. Discomfort in taste: Gp A: 5/39, GP B: 7/39, Gp C: 0, Gp D: 1/39. Discomfort in sensibility: Gp A: 2/39, Gp B: 2/39, Gp C: 0, Gp D: 0. Gingival damage: Gp A: 0, Gp B: 0, Gp C: 3/39, Gp D: 0. Stain on teeth/tongue: Gp A: 4/39, Gp B: 9/39, Gp C: 0 , Gp D: 0 . Mouth burning during application: Gp A: 7/39, Gp B: 5/39, Gp C: 0, Gp D: 0. Bleeding of the gingiva: Gp A: 0, Gp B: 0, Gp C: 0, Gp D: 1 . White plaque on tongue immediately after use: Gp A: 0, Gp B: $1 / 39$, Gp C: 0, Gp D: 0

- At 8 weeks: no side effects: Gp A: 19/39, Gp B: 10/39, Gp C: 38/39, Gp D: 39/39. Discomfort in taste: Gp A: 6/39, GP B: 4/39, Gp C: 0, Gp D: 0. Discomfort in sensibility: Gp A: 2/39, Gp B: 1/39, Gp C: 0, Gp D: 0. Gingival damage: Gp A: 0, Gp B: 0, Gp C: 1/39, Gp D: 0. Stain on teeth/tongue: Gp A: 6/39, Gp B: 19/39, Gp C: 0 , Gp D: 0 . Mouth burning during application: Gp A: 6/39, Gp B: 4/39, Gp C: 0, Gp D: 0. Bleeding of the gingiva: Gp A: 0, Gp B: 0, Gp C: 0, Gp D: 0 . White plaque on tongue immediately after use: Gp A: 0, Gp B: $1 / 39$, Gp C: 0, Gp D: 0

Smokers: 33 (21.15\%) overall. By group: Gp A: 10\%; Gp B: 31\%; Gp C: 28\%; Gp D: 15\%

Declarations/conflicts of interest: not reported

\section{Risk of bias}

Bias Authors' judgement Support for judgement

Random sequence genera- Low risk tion (selection bias)
Quote: ".. the 156 participants were randomly assigned to four groups with 39 subjects in each group.. In a box containing 156 envelopes in four strata (three strata with 40 and one with 36), each participant had to draw one envelope containing the number of the attributed product"

Comment: drawing of lots is an adequate method of random sequence generation

Allocation concealment Low risk
(selection bias)

Quote: ".. the 156 participants were randomly assigned to four groups with 39 subjects in each group.. In a box containing 156 envelopes in four strata (three strata with 40 and one with 36), each participant had to draw one envelope containing the number of the attributed product"

Quote: "The assignment of subjects to groups was performed by a person not involved in the examination"

Comment: allocation of subjects to groups was concealed up to the point that participants drew an envelope and were allocated to a group

\begin{tabular}{|c|c|c|}
\hline $\begin{array}{l}\text { Blinding of participants } \\
\text { and personnel (perfor- } \\
\text { mance bias) } \\
\text { All outcomes }\end{array}$ & High risk & $\begin{array}{l}\text { Comment: participants were not blinded. The different interventions meant } \\
\text { that participants would know which group they were in and this could have af- } \\
\text { fected their oral health behaviours and hence the outcome. The direction of } \\
\text { this potential bias is not clear }\end{array}$ \\
\hline
\end{tabular}

Blinding of outcome as- Low risk sessment (detection bias) All outcomes
Quotes: "The study was conducted by a blinded operator.." and "Because clinically visible side effects... might have influenced examiner blinding, an additional statistical analysis was performed for subjects without visible side effects. Again, compared to all subjects, only small discrepancies of this subgroup and no changes in statistical significance were found. This finding indicates that clinically visible side effects did not affect examiner accuracy"

Comment: aAlthough a risk of bias of outcome assessment due to staining of teeth/tongue in both active mouthrinse groups was likely, it did not appear to influence the results

Incomplete outcome data Low risk All randomised participants were included in the analyses

(attrition bias) 
Zimmer 2006 (Continued)

All outcomes

Selective reporting (re- High risk porting bias)
Adverse effects were reported with no double counting - if any participant experienced more than 1 adverse effect, only the 'most relevant' one was reported. No information is provided on how the 'most relevant' side effect was chosen to be reported and information about participants who experienced more than 1 adverse effect is not reported
Quote: "Before starting the study the intraexaminer reliability was tested by repeated measurements of MPPI, QHI, and PBI and analysed by Cohen's $k$ test. the results were as follows: $\mathrm{MPPI}=0.86, \mathrm{QHI}=0.82$ and $\mathrm{PBI}=0.88$." No other apparent bias identified

Quote: "All examinations were conducted by one single examiner (GK)"

Smokers: CHX NaF (4) $10.26 \%$, control (6) $15.38 \%$. The authors state that this difference failed to reach statistical significance $(P=0.076)$

\section{Study characteristics}

Methods
Location: Dental School of the Witten/Herdecke University, Witten, Germany
Number of centres: 1
Study duration: quote: "Subjects were recruited between April 20th, 2010 and June16th, 2010." Study
duration and duration of rinsing was 8 weeks
duration and duration of rinsing was 8 weeks

\section{Participants}

\section{Participants: healthy adults}

Inclusion criteria: quotes: "subjects aged 18-65 years and having a mean PBI per tooth $\geq 0.5$ were enrolled in the study" and "... Informed consent was obtained by each subject. Each subject had a minimum of 20 permanent natural teeth. Third molars, orthodontically banded, fully crowned or extensively restored or abutment teeth were not included in the tooth count"

Exclusion criteria: quote: "Subjects were excluded from the study if they were pregnant or breast-feeding, had diabetes type I or II, or severe periodontal disease, or wore removable dentures. Severe periodontal disease was defined as the presence of clinical attachment loss of more than $5 \mathrm{~mm}$ according to the periodontal disease classification of the American Academy of Periodontology.... However, a subject was excluded only if this applied for a minimum of three teeth. Subjects were also excluded if any of the following conditions was present: use of antibiotics within 2 weeks prior the first examination or use of any systemic medication which would have an effect on gingival conditions within 30 days prior to the screening visit, or recent history (within the last year) of alcohol or other substance abuse. Dental professionals, dental students and employees of the sponsor or members of their immediate families were also excluded from the study"

Baseline gingivitis: (Papillary Bleeding Index) Gp A: mean 1.06 (SD 0.4); Gp B: mean 0.99 (SD 0.37); Gp C: mean 0.94 (SD 0.31); Gp D: mean 1.02 (SD 0.39).

Age (years): overall: mean 33.9. Gp A: mean 33.8; Gp B: mean 34.0; Gp C: mean 33.9; Gp D: mean 33.8. Range 18-65 years

Gender: $n$ t reported

Number randomised: 160 (Gp A: 40; Gp B: 40; Gp C: 40; Gp D: 40)

Number evaluated: 155 (Gp A: 39; Gp B: 39; Gp C: 40; Gp D: 37) 
Zimmer 2015 (Continued)

Interventions

\begin{abstract}
Comparison: (twice daily toothbrushing and $0.06 \% \mathrm{CHX}$ digluconate $+0.025 \% \mathrm{~F}$ as sodium fluoride mouthrinse) versus (twice daily toothbrushing and an experimental alcohol-free mouthrinse with $\mathbf{0 . 0 6} \% \mathrm{CHX}$ digluconate $\mathbf{0 . 0 2 5} \% \mathrm{~F}$ as sodium fluoride) versus (twice daily toothbrushing alone) versus (twice daily toothbrushing and an experimental alcohol-free mouthrinse with $0.06 \%$ CHX digluconate $+0.03 \%$ cetylpyridinium chloride (CPC) $+0.025 \% \mathrm{~F}$ as sodium fluoride) ${ }^{\text {* }}$
\end{abstract}

$\mathrm{Gp} \mathrm{A}(\mathrm{n}=40)$ : twice daily toothbrushing and rinsing twice a day for 30 seconds with $10 \mathrm{~mL}$ of an alcohol-containing mouthrinse with $0.06 \% \mathrm{CHX}$ digluconate $+0.025 \%$ fluoride as sodium fluoride for 8 weeks

Gp B ( $n=40)$ : twice daily toothbrushing and rinsing twice a day for 30 seconds with $10 \mathrm{~mL}$ of an experimental alcohol-free mouthrinse with $0.06 \% \mathrm{CHX}$ digluconate $+0.025 \%$ fluoride as sodium fluoride for 8 weeks

Gp C ( $n=40)$ : twice daily toothbrushing alone

${ }^{*} \mathrm{Gp} \mathrm{D}(\mathrm{n}=40)$ : twice daily toothbrushing and rinsing twice a day for 30 seconds with $10 \mathrm{~mL}$ of an experimental alcohol-free mouthrinse with $0.06 \% \mathrm{CHX}$ digluconate $+0.03 \%$ cetylpyridinium chloride (CPC) + $0.025 \%$ fluoride as sodium fluoride. We excluded this arm from our risk of bias and analysis

Prophylaxis at baseline: not reported

OHI: quotes: "All participants received a short instruction on how to conduct the attributed oral hygiene measurements. The subjects were advised to brush their teeth in the morning and in the evening postprandial in the usual manner. No instructions concerning brushing technique and brushing duration were given. After using the brush, the subjects rinsed their mouth with tap water" and "During the study period, the use of other than the attributed oral hygiene tools strictly was prohibited... Interproximal cleaning devices had been permitted if they were part of the usual oral hygiene routine of the included subjects"

Timing of toothbrushing in relation to mouthrinsing: quote: "The subjects of the rinsing groups waited for $30 \mathrm{~min}$. Thereafter, they rinsed for $30 \mathrm{~s}$ with $10 \mathrm{ml}$ of the assigned rinse. Afterwards, the subjects refrained from drinking, eating and rinsing for at least $30 \mathrm{~min}$. To control the waiting and the rinsing time, each subject was provided with a digital stop watch"

Outcomes Gingivitis (gingival bleeding: Papillary Bleeding Index, Saxer 1975); plaque (TQH, Turesky 1970; Modified Proximal Plaque Index, Zimmer 2005) assessed at 4 and 8 weeks follow-up. All adverse events occurring during the study period were recorded using a questionnaire and by clinical examination. Occurrence of discomfort in taste, discomfort in sensibility, gingival damage, gingival bleeding, staining of teeth and tongue, mouth burning and white plaque on tongue immediately after use was registered at baseline and after 4 and 8 weeks. Oral soft tissue examination was performed at each visit

Funding

Quote: "This study was funded by GlaxoSmithKline Consumer Healthcare (Bühl, Germany). GlaxoSmithKline was involved in the design and conduct of the study and provided logistical support during the trial. Data management and statistical analysis were performed by an independent institute at the Heinrich-Heine-University Düsseldorf. The manuscript was prepared by Prof. Zimmer, Dr. Naumova and Dr. Jordan. GlaxoSmithKline was permitted to review the manuscript and suggest changes, but the final decision on content was exclusively retained by the authors"

Notes Sample size calculation: quote: "... was calculated on the basis of the following assumptions: power 0.8; alpha error: 0.05; delta-QHI between groups after 8 weeks: 0.3 (SD 0.5); one tailed analysis (Axum 7.0)"

Adverse effects: tooth staining at 8 weeks: Gp A: $18 / 39$, Gp B: 21/39 and Gp C: $8 / 40$. Tongue staining at 8 weeks: Gp A: 18/39, Gp B: 22/39 and Gp C: $14 / 40$

Gastrointestinal adverse events were found in all groups (Gp 1 (A): 1 case, Gp 2 (B): 2 cases, Gp 3 (no data extracted): 7 cases, Gp 4 (C): 1 case); in 5 cases ( 1 in Gp 2 (B) and 4 in Gp 3 (no data extracted)), gastrointestinal infection was given as reason; in 4 cases, diarrhoea; and in 1 case, 'stomach burning' and meteorism, respectively. No serious adverse events were observed in the study

Smokers by group: Gp A: 10/40 (25\%); Gp B: $9 / 40$ (23\%); Gp C: 10/40 (25\%) (differences between the groups were not statistically significant) 
The trial was registered at the Clinical Trials Register of the National Institutes of Health (NCT01811615, www.clinicaltrials.gov)

Declarations/conflicts of interest: none stated

Tooth staining data for groups A and B (at 4 weeks) were combined in the main analysis

\section{Risk of bias}

\begin{tabular}{lll}
\hline Bias & Authors' judgement & Support for judgement \\
\hline $\begin{array}{ll}\text { Random sequence genera- } \\
\text { tion (selection bias) }\end{array}$ & Low risk & $\begin{array}{l}\text { Quote: "Using the stratification by sex and } \mathrm{PBI}(\mathrm{PBI} / \text { tooth } \geq 0.5 \text { and }<1.0 \text { or } \mathrm{PBI} / \\
\text { tooth } \geq 1.0) \text {, the } 160 \text { eligible participants were allocated by block randomiza- } \\
\text { tion to one of four groups with } 40 \text { subjects each. Block randomization was per- } \\
\text { formed by a statistician not involved in the study" }\end{array}$ \\
& & Comment: adequate method of random sequence generation
\end{tabular}

\begin{tabular}{ll}
\hline $\begin{array}{l}\text { Allocation concealment } \\
\text { (selection bias) }\end{array}$ & Quotes: "Subjects within each group were randomly assigned to one of the fol- \\
& $\begin{array}{l}\text { lowing groups" and "Block randomization was performed by a statistician not } \\
\text { involved in the study" }\end{array}$
\end{tabular}

Comment: we consider it unlikely that participants and investigators enrolling participants could foresee assignment

\begin{tabular}{|c|c|c|}
\hline $\begin{array}{l}\text { Blinding of participants } \\
\text { and personnel (perfor- } \\
\text { mance bias) } \\
\text { All outcomes }\end{array}$ & High risk & $\begin{array}{l}\text { Quotes: "All personal instructions and delivering of the study products were } \\
\text { performed by a study nurse not involved (A.P.) in the study examinations. The } \\
\text { subjects were forbidden to tell the examiner their regimen during the study } \\
\text { visits" and "With respect to tooth staining, chi square-test revealed significant } \\
\text { more occurrences in the three rinsing groups when compared to the negative } \\
\text { control }(\mathrm{P}<0.001) \text { " }\end{array}$ \\
\hline
\end{tabular}

Comment: due to the design of the study the participants in toothbrushing only group could not be blinded. The higher incidence of staining in the $\mathrm{CHX}$ groups means that blinding may not be possible in a comparison against the control

Blinding of outcome as-
sessment (detection bias)

All outcomes

The study was designed to be examined blind. Quotes: "All assessments of an individual subject in the course of the study have been conducted by the same investigator (P.K.) who had experience from previous clinical studies" and "with respect to tooth staining, chi square-test revealed significantly more occurrences in the rinsing groups when compared to the negative control $(\mathrm{P}<0.001)^{\prime \prime}$

Comment: the higher incidence of staining in the $\mathrm{CHX}$ groups means that blinding may not be possible in a comparison against the control

\begin{tabular}{lll}
$\begin{array}{l}\text { Incomplete outcome data } \\
\begin{array}{l}\text { (attrition bias) } \\
\text { All outcomes }\end{array}\end{array}$ & Low risk & $\begin{array}{l}\text { Attrition 2/120 (1.7\%). By group: Gp A: 1/40 (0.03\%); Gp B: 1/40 (0.03\%); Gp C: } \\
\text { no losses. Reasons for losses: Gp A: informed consent withdrawn; Gp B: proto- } \\
\text { col violation } \\
\text { Comment: attrition is minimal and unlikely to affect the outcome }\end{array}$ \\
\hline $\begin{array}{l}\text { Selective reporting (re- } \\
\text { porting bias) }\end{array}$ & Low risk & $\begin{array}{l}\text { All outcomes stated in the methods sections were reported in the results sec- } \\
\text { tion }\end{array}$ \\
\hline $\begin{array}{l}\text { Other bias } \\
\text { Unclear risk }\end{array}$ & $\begin{array}{l}\text { No mention of intraexaminer reproducibility } \\
\text { Groups were balanced at baseline for age and smoking status, gingivitis and } \\
\text { plaque }\end{array}$
\end{tabular}


$\mathrm{ASF}=$ amine fluoride and stannous fluoride $\mathrm{BOP}=$ bleeding on probing; $\mathrm{CAL}=$ clinical attachment level; $\mathrm{CHX}=\mathrm{chlorhexidine} ; \mathrm{Cl}=\mathrm{Cal}$ culus Index; $\mathrm{Cl}=$ confidence interval; $\mathrm{CSSI}=$ Calculus Surface Severity Index; $\mathrm{DI}=$ Discolouration Index; $\mathrm{F}=$ fluoride; $\mathrm{Gp}=$ group; $\mathrm{GI}=\mathrm{Gingival}$ Index; $\mathrm{ITT}=$ intention-to-treat analysis; $\mathrm{MSE}=$ mean squared error; $\mathrm{OH}=$ oral hygiene; $\mathrm{OHI}=$ oral hygiene instruction; $\mathrm{PAL}=$ probing attachment level; PBS = Papillary Bleeding Score; PD = pocket depth; PI = Plaque Index; PMGI = Papillary Marginal Gingival Index; PPD = probing pocket depth; ppm = parts per million; PVP = polyvinyl pyrrolidone; SD = standard deviation; $\mathrm{SE}$ = standard error; $\mathrm{SES}$ = socioeconomic status; $\mathrm{SRP}$ = scaling and root planing; TQH = Turesky modification of the Quigley and Hein Index; VAS = visual analogue scale.

\section{Characteristics of excluded studies [ordered by study ID]}

\section{Study Reason for exclusion}

\section{Bay 1975}

\section{Biswas 2014}

Bouwsma 1992

Brown 2002

\begin{tabular}{ll}
\hline Caton 1993 & $\begin{array}{l}\text { The } \mathrm{CHX} \text { group received } \mathrm{OH} \text { instructions but the control group did not. The mechanical OH proce- } \\
\text { dures were potentially not the same in both groups }\end{array}$ \\
\hline Cortelli 2015 & $\begin{array}{l}\text { The } \mathrm{CHX} \text { arms used } \mathrm{CHX} \text { gel (tongue brushing for } 1 \text { min after rinsing) in addition to } \mathrm{CHX} \text { rinse. We } \\
\text { were unable to separate the effect of the CHX rinse from the effect of the other active agent }\end{array}$ \\
\hline CTRI 2014 & $\begin{array}{l}\text { Comparison is with another active rinse and there is no placebo/control or mechanical OH only } \\
\text { group }\end{array}$ \\
\hline Ernst 1998 & $\begin{array}{l}\text { Comparison is with another active rinse and there is no placebo/control or mechanical OH only } \\
\text { group }\end{array}$ \\
\hline Eshwar 2016 & $\begin{array}{l}\text { Comparison is with another active rinse and there is no placebo/control or mechanical OH only } \\
\text { group }\end{array}$ \\
\hline Fischman 1975 & $\begin{array}{l}\text { This study compared CHX, zinc and placebo mouthrinses but each rinse group was further split into } \\
\text { those who received a professional prophylaxis prior to the start of the study and those that did not. } \\
\text { The study outcomes were not reported in a format that could be used in the review and data were } \\
\text { not available from the authors of the study }\end{array}$
\end{tabular}

It is unclear from the published report whether the study is a randomised trial. Attempts to contact the first author for clarification were unsuccessful

Comparison is with another active rinse and there is no placebo/control or mechanical OH only group

The test group used a triangular wooden interdental cleaner once daily after toothbrushing which the $\mathrm{CHX}$ group did not use. The mechanical $\mathrm{OH}$ procedures were not the same in both groups

The study compares $\mathrm{CHX}$ plus mechanical $\mathrm{OH}$ and 2-monthly professional prophylaxis versus mechanical $\mathrm{OH}$ and 6-monthly check up and professional prophylaxis (standard care). The mechanical $\mathrm{OH}$ procedures were not the same in both groups

\section{Goutham 2013}

It is unclear from the published report whether the study is a randomised trial. Attempts to contact the first author for clarification were unsuccessful

\begin{tabular}{ll} 
Gupta 2014 & Concern about inclusion of duplicated outcome data from another study by the same authors \\
\hline Gupta 2015 & Concern about inclusion of duplicated outcome data from another study by the same authors \\
\hline Gupta 2015a & $\begin{array}{l}\text { Concern about inclusion of duplicated outcome data from other studies in other published trials } \\
\text { from these researchers }\end{array}$
\end{tabular}




\begin{tabular}{ll}
\hline Study & Reason for exclusion \\
\hline Haffajee 2009 & $\begin{array}{l}\text { Comparison is with another active rinse and there is no placebo/control or mechanical OH only } \\
\text { group }\end{array}$
\end{tabular}

\begin{tabular}{ll}
\hline Karim 2014 & Concern about inclusion of duplicated outcome data from another study by the same authors \\
\hline Lang 1982 & $\begin{array}{l}\text { It is unclear from the published report whether the study is a randomised trial. Attempts to contact } \\
\text { the first author for clarification were unsuccessful }\end{array}$ \\
\hline Leyes Borrajo 2002 & $\begin{array}{l}\text { It is unclear from the published report whether the study is a randomised trial. Additional informa- } \\
\text { tion obtained from the contact author indicate quasi-randomisation (participants were assigned to } \\
\text { groups according to the order that they came to the university) }\end{array}$ \\
\hline
\end{tabular}

Luoma $1978 \quad$ CHX, F and placebo groups also used a toothpaste at evenings and weekend with the same compo-
sition as their respective rinse solution. Therefore the $\mathrm{CHX}$ rinse group also used a $\mathrm{CHX}$-containing toothpaste. We were unable to separate the effect of the $\mathrm{CHX}$ rinse from the effect of the other active agent

\begin{tabular}{|c|c|}
\hline Madden 2008 & $\begin{array}{l}\text { The } \mathrm{CHX} \text { group received a more intensive intervention including 2-monthly } \mathrm{OH} \text { instruction com- } \\
\text { pared to the control group who received 6-monthly } \mathrm{OH} \text { alone. The mechanical } \mathrm{OH} \text { procedures were } \\
\text { (potentially) not the same in both groups }\end{array}$ \\
\hline McKenzie 1992 & $\begin{array}{l}\text { Comparison is with another active rinse and there is no placebo/control or mechanical OH only } \\
\text { group }\end{array}$ \\
\hline Nadkerny 2015 & Duration of rinsing was 15 days \\
\hline NCT01750801 & $\begin{array}{l}\text { Comparison is with another active rinse and there is no placebo/control or mechanical } \mathrm{OH} \text { only } \\
\text { group }\end{array}$ \\
\hline NCT02546804 & $\begin{array}{l}\text { Comparison is with another active rinse and hot salt mouthwash and there is no placebo/control or } \\
\text { mechanical } \mathrm{OH} \text { only group }\end{array}$ \\
\hline Ousehal 2011 & $\begin{array}{l}\text { It is unclear from the published report whether the study is a randomised trial. Attempts to contact } \\
\text { the first author for clarification were unsuccessful }\end{array}$ \\
\hline Paknejad 2006 & $\begin{array}{l}\text { It is unclear from the published report whether the study is a randomised trial. Attempts to contact } \\
\text { the first author for clarification were unsuccessful }\end{array}$ \\
\hline Persson 1991 & $\begin{array}{l}\text { Comparison is with another active rinse and there is no placebo/control or mechanical OH only } \\
\text { group }\end{array}$ \\
\hline Persson 1998 & $\begin{array}{l}\text { The } \mathrm{CHX} \text { group received a more intensive intervention including cognitive behavioural education ( } 2 \\
\text { hours) compared to the control group who received standard care }\end{array}$ \\
\hline Priya 2015 & $\begin{array}{l}\text { Comparison is with another active rinse and there is no placebo/control or mechanical OH only } \\
\text { group }\end{array}$ \\
\hline Quirynen 2005 & $\begin{array}{l}\text { The study involves } 1 \text {-stage full-mouth disinfection involving use of } \mathrm{CHX} \text { gel at baseline in addition } \\
\text { to the mouthrinses. We were unable to separate the effect of the } \mathrm{CHX} \text { rinse from the effect of the } \\
\text { other active agent }\end{array}$ \\
\hline Quirynen 2006 & $\begin{array}{l}\text { The study involves 1-stage full-mouth disinfection involving use of } \mathrm{CHX} \text { gel at baseline and } 1 \text { week } \\
\text { in addition to the mouthrinses. We were unable to separate the effect of the } \mathrm{CHX} \text { rinse from the ef- } \\
\text { fect of the other active agent }\end{array}$ \\
\hline
\end{tabular}




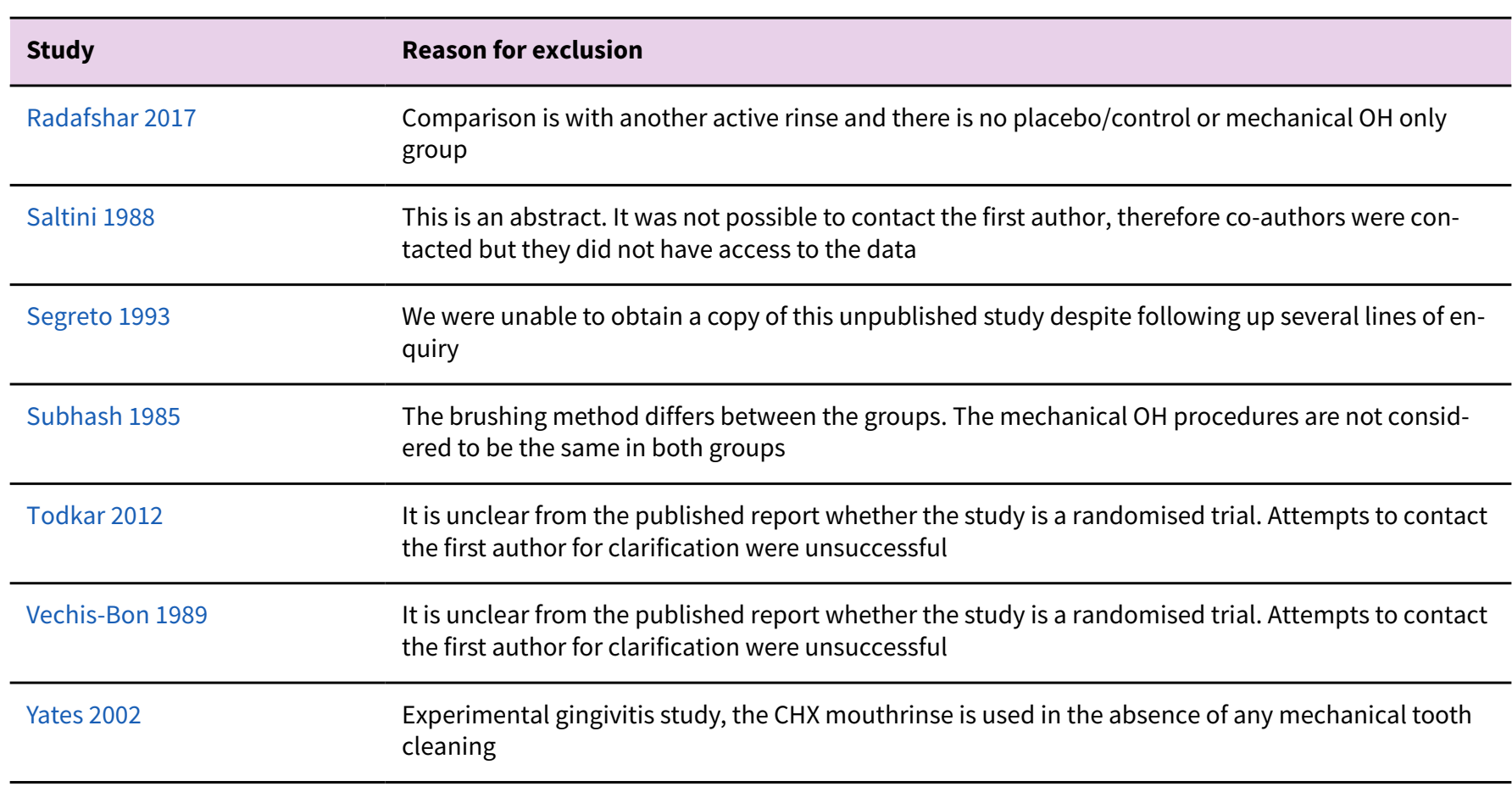

$\mathrm{CHX}=$ chlorhexidine $\mathrm{OH}=$ oral hygiene $\mathrm{F}=$ fluoride .

Characteristics of studies awaiting classification [ordered by study ID]

CTRI/2011/05/001774

Study design: parallel group 3 arms
Location: Apollo Hospital, Dental and Facial Surgical Centre, RV Dental College, Santosh Hospital,
Bangalore, Karnataka, India
Number of centres: appears to be 4
Study duration: $7 / 8$ months. Duration of rinsing appears to be 6 months

Interventions
Chlorhexidine $0.12 \%$ (Orocleanse) with alcohol versus chlorhexidine $0.12 \%$ (Oroclear) without alcohol versus placebo in conjunction with regular self-performed oral hygiene measures

Rinsing with $15 \mathrm{~mL}$ twice daily for 30 seconds for 6 months
Gingival inflammation: GI, also stain indices

Brief summary of results mentions $\mathrm{PI}, \mathrm{PBI}$ and $\mathrm{Cl}$ not specified in methods

Study completed. Brief summary of results reported but no outcome data are reported. We contacted the author for further information but did not receive a reply 
Study duration: duration of rinsing 60 days

Participants
Periodontics, Government Dental College and Research Institute, Bangalore from January 2013 to
April 2013
Quote: "..instructed not to rinse/eat anything for 30 min after mouthwash use. Subjects were al-
so asked to refrain from all other unassigned forms of oral hygiene aids, including dental floss and
chewing gum during the study. No oral hygiene instructions like brushing and flossing were given
to the subjects to exclude the influence of improved oral hygiene practices on the results"
wash. Subjects were instructed to use $15 \mathrm{~mL}$ of mouthwash twice daily

Outcomes
$\mathrm{PI}, \mathrm{GI}$ and $\mathrm{OHI}-\mathrm{S}$, and microbiological colony counts were recorded on baseline and 7, 30 and 60
days respectively

\section{NCT02065414}

Study design: parallel, (3 arms - 2 arms are relevant to this review)
Location: not clear "Clinical Centre"
Number of centres: 1
Study duration: 4 weeks

\section{Participants}

Interventions
Participants: aged 18 years and older with gingivitis, but without moderate/advanced periodontitis

Chlorhexidine rinse (Corsodyl) $0.12 \%$ versus experimental mouthrinse (Listerine Advance Gum Defense) versus placebo

Chlorhexidine: rinse with $10 \mathrm{~mL}$ for 60 seconds twice daily for 4 weeks ( 5 min after brushing and rinsing mouth with water)

Experimental rinse: rinse with $20 \mathrm{~mL}$ for 30 seconds twice daily for 4 weeks (immediately after brushing and rinsing mouth with water)

Placebo: rinse with $20 \mathrm{~mL}$ for 30 seconds twice daily for 4 weeks (immediately after brushing and rinsing mouth with water)

\section{Outcomes}

Gingivitis: gingival inflammation, Modified Gingival Index at 4 weeks; gingival bleeding, Gingival Bleeding Index at 1 and 4 weeks. Plaque: Turesky Modification of the Quigley and Hein Plaque Index at 4 weeks. Extrinsic tooth stain: Macpherson Modification of the Lobene Stain Index at 4 weeks

\section{Notes}

Study completed. Contacted investigator for further details but did not receive a reply 
Methods

Participants
Trial design: parallel, ( 3 arms - 2 arms relevant to this review) (study number 2 only, study number 1 does not meet the inclusion criteria for the review)

Location: Periodontology Department, Mashad Dental School, Iran

Number of centres: assume 1 centre

Study duration: recruitment period not stated. Duration of rinsing was 4 weeks and the outcome was assessed 6 weeks after scaling and root planing

Participants: outpatients with chronic periodontitis referred to the Periodontology Department, Mashad Dental School, Iran

Inclusion criteria: chronic periodontitis with pocketing and attachment loss in all quadrants (over 45 years old)

Exclusion criteria: "Patients on antibiotic therapy or anti-inflammatory drugs, those with any history of systemic disease, or allergy to components of the mouthwash, those who had undergone any form of non-surgical or surgical periodontal therapy in the last 6 months, pregnant and lactating mothers and smokers were excluded"

Baseline gingivitis: BOP, Group A (66.8 \pm 14.85$)$; Group B (71.08 \pm 10.23$)$; Group C (57.18 \pm 19.47$)$

Age at baseline (years): not reported. Over 45 years old

Gender: not reported

Number randomised: 30 (number randomised to each group not reported, assumed 10 in each group at baseline)

Number evaluated: not reported

Interventions

Chlorhexidine rinse versus herbal rinse ${ }^{\star}$ versus control rinse

Group A (assume $\mathrm{n}=10$ ): CHX (concentration not reported) $10 \mathrm{~mL}, 1 \mathrm{~min}$, twice daily

*Group B (assume $\mathrm{n}=10$ ): herbal mouthwash ( $S$ alba, M sylvestris and A officinalis) $10 \mathrm{~mL}, 1 \mathrm{~min}$, twice daily. (We excluded this arm from our risk of bias and analysis)

Group C (assume $\mathrm{n}=10$ ): control (normal saline) $10 \mathrm{~mL}, 1 \mathrm{~min}$, twice daily

Prophylaxis at baseline: quote: "Scaling and root planing was accomplished in two visits for all patients and local anaesthesia was used when required"

OHI: not reported

Non-supervised rinsing

Timing of mouthrinsing in relation to toothbrushing: not reported

Postrinsing instructions: quote: "Subsequent rinsing with water was not allowed"

Duration of rinsing: 4 weeks

Outcomes

Gingivitis: BOP (no reference), PD, CAL were measured using a periodontal probe at baseline and then 6 weeks after scaling and root planing

Notes

Sample size calculation: not reported

Adverse effects: quote: "No adverse reaction was seen in the herbal mouthwash group, however, in the $\mathrm{CHX}$ group there were some adverse reactions such as dental and tongue staining" 
It is not clear exactly when the outcome was assessed. Outcome assessment appears to have been completed 2 weeks after rinsing ceased

We are awaiting clarification from the authors about various aspects of this study

$\mathrm{BOP}=$ bleeding on probing; $\mathrm{CAL}=$ clinical attachment level; $\mathrm{CHX}=$ chlorhexidine $\mathrm{Cl}=$ Calculus Index; $\mathrm{GI}=\mathrm{Gingival}$ Index; $\mathrm{OHI}=$ oral hygiene instruction; $\mathrm{OHI}-\mathrm{S}=$ Oral Hygiene Index-Simplified; $\mathrm{PBI}=$ Papillary Bleeding Index; $\mathrm{PD}=$ pocket depth; $\mathrm{PI}=\mathrm{Plaque}$ Index.

Characteristics of ongoing studies [ordered by study ID]

\section{NCT02709785}

Study name

Methods
SmartMouth Advanced Clinical Formula clinical research design protocol

Trial design: parallel, (3 arms - 2 arms relevant to this review)

Location: Saint Louis University Center for Advanced Dental Education, Saint Louis, Missouri, USA and Southern Illinois University School of Dental Medicine, Alton, Illinois, USA

Number of centres: 2

Study duration: March 2016 to June 2017. Study duration 10 weeks. Duration of rinsing 6 weeks
Participants: adults with gingivitis or chronic periodontitis

Inclusion criteria: subjects between the ages of 18 to 80 , with a diagnosis of gingivitis or chronic periodontitis, mean $\mathrm{GI} \geq 0.4$ and $\mathrm{PI} \geq 1.0$, subjects that are in good medical health at time of the study, at least 20 permanent natural teeth

Exclusion criteria: use of local or systemic antibiotics during the course of the study, subjects that are pregnant or nursing mothers, undergoing orthodontic therapy, subjects wearing removable prostheses, subjects taking medication which is altering the gingiva or causing inflammation, gingival overgrowth, history of sensitivity or suspected allergies following the use of oral hygiene products, subjects taking anti-inflammatory or anticoagulant medications that would alter the gingiva and promote bleeding, subjects that require antibiotic prophylaxis prior to dental treatment, subjects that have acute dental problems requiring immediate treatment

Baseline gingivitis: $\mathrm{GI} \geq 0.4$ (Löe \& Silness 1963)

Age at baseline: $18-80$ years are eligible

Prophylaxis: all subjects will be given a complete dental prophylaxis to remove plaque, calculus, and stain

$\mathrm{OHI}$ : written and verbal $\mathrm{OHI}$ will be given that include a regular regimen of brushing twice daily and flossing daily. All subjects will be assigned a commercially available ADA-accepted toothbrush and dental floss

Number to be randomised: 85 (each group will have at least 25 subjects)
$0.12 \%$ chlorhexidine rinse versus SmartMouth Advanced Clinical Formula (ACF) rinse versus placebo rinse

Outcomes

Gingivitis: gingival inflammation: GI Löe \& Silness 1963; gingival bleeding (GI scores 2 and 3); Turesky modification of the Quigley and Hein Plaque Index, Turesky 1970. Extrinsic tooth staining: modification of the Lobene Tooth Stain Index, Lobene 1968. Supragingival calculus on the lingual surfaces of the 6 mandibular anterior teeth will be scored using the Volpe-Manhold Calculus Index, Volpe 1965. Safety assessments and assessment of the oral soft tissues will be conducted at each 
NCT02709785 (Continued)

visit by visual examination of the oral cavity. Evaluation of taste perception, malodour via questionnaire

\begin{tabular}{ll}
\hline Starting date & March 2016 \\
\hline Contact information & $\begin{array}{l}\text { Contact: David D Miley, Saint Louis University Center for Advanced Dental Education, Saint Louis, } \\
\text { Missouri, USA }\end{array}$ \\
\hline Notes & Sponsor: Saint Louis University \\
& Collaborator: Triumph Pharmaceuticals \\
& NCT02709785 \\
\hline
\end{tabular}

$\mathrm{ADA}=$ American Dental Association; $\mathrm{GI}=$ Gingival Index; $\mathrm{PI}=$ Plaque Index; $\mathrm{OHI}=$ oral hygiene instruction .

\section{DATA AND ANALYSES}

\section{Comparison 1. CHX versus placebo/control mouthrinse or no mouthrinse}

\begin{tabular}{|c|c|c|c|c|}
\hline Outcome or subgroup title & No. of studies & $\begin{array}{l}\text { No. of partici- } \\
\text { pants }\end{array}$ & Statistical method & Effect size \\
\hline $\begin{array}{l}1.1 \text { Gingival Index (0-3) 4-6 } \\
\text { weeks }\end{array}$ & 10 & 805 & $\begin{array}{l}\text { Mean Difference (IV, Random, 95\% } \\
\mathrm{Cl} \text { ) }\end{array}$ & $-0.21[-0.31,-0.11]$ \\
\hline 1.1.1 CHX versus no rinse & 2 & 339 & $\begin{array}{l}\text { Mean Difference (IV, Random, 95\% } \\
\mathrm{Cl})\end{array}$ & $-0.13[-0.31,0.05]$ \\
\hline $\begin{array}{l}\text { 1.1.2 CHX versus place- } \\
\text { bo/control rinse }\end{array}$ & 8 & 466 & $\begin{array}{l}\text { Mean Difference (IV, Random, 95\% } \\
\mathrm{CI})\end{array}$ & $-0.23[-0.34,-0.13]$ \\
\hline $\begin{array}{l}1.2 \text { Gingival Index (0-3) } 6 \\
\text { months }\end{array}$ & 13 & 2616 & $\begin{array}{l}\text { Mean Difference (IV, Random, 95\% } \\
\mathrm{Cl} \text { ) }\end{array}$ & $-0.20[-0.30,-0.11]$ \\
\hline 1.2.1 $\mathrm{CHX}$ versus no rinse & 2 & 142 & $\begin{array}{l}\text { Mean Difference (IV, Random, 95\% } \\
\mathrm{Cl} \text { ) }\end{array}$ & $-0.12[-0.18,-0.05]$ \\
\hline $\begin{array}{l}\text { 1.2.2 CHX versus place- } \\
\text { bo/control rinse }\end{array}$ & 11 & 2474 & $\begin{array}{l}\text { Mean Difference (IV, Random, 95\% } \\
\mathrm{Cl} \text { ) }\end{array}$ & $-0.22[-0.33,-0.11]$ \\
\hline $\begin{array}{l}1.3 \text { Gingival bleeding } 4-6 \\
\text { weeks }\end{array}$ & 8 & 649 & $\begin{array}{l}\text { Std. Mean Difference (IV, Random, } \\
95 \% \mathrm{Cl} \text { ) }\end{array}$ & $-0.56[-0.79,-0.33]$ \\
\hline 1.3.1 CHX versus no rinse & 4 & 459 & $\begin{array}{l}\text { Std. Mean Difference (IV, Random, } \\
95 \% \mathrm{CI} \text { ) }\end{array}$ & $-0.69[-0.89,-0.50]$ \\
\hline $\begin{array}{l}\text { 1.3.2 CHX versus place- } \\
\text { bo/control rinse }\end{array}$ & 4 & 190 & $\begin{array}{l}\text { Std. Mean Difference (IV, Random, } \\
95 \% \mathrm{CI} \text { ) }\end{array}$ & $-0.36[-0.77,0.06]$ \\
\hline $\begin{array}{l}1.4 \text { Gingival bleeding } 6 \\
\text { months }\end{array}$ & 8 & 1132 & $\begin{array}{l}\text { Std. Mean Difference (IV, Random, } \\
95 \% \mathrm{Cl} \text { ) }\end{array}$ & $-0.72[-1.02,-0.42]$ \\
\hline 1.4.1 CHX versus no rinse & 2 & 142 & $\begin{array}{l}\text { Std. Mean Difference (IV, Random, } \\
95 \% \mathrm{Cl} \text { ) }\end{array}$ & $-0.49[-0.83,-0.16]$ \\
\hline
\end{tabular}




\begin{tabular}{|c|c|c|c|c|}
\hline Outcome or subgroup title & No. of studies & $\begin{array}{l}\text { No. of partici- } \\
\text { pants }\end{array}$ & Statistical method & Effect size \\
\hline $\begin{array}{l}\text { 1.4.2 CHX versus place- } \\
\text { bo/control rinse }\end{array}$ & 6 & 990 & $\begin{array}{l}\text { Std. Mean Difference (IV, Random, } \\
95 \% \mathrm{Cl} \text { ) }\end{array}$ & $-0.79[-1.16,-0.41]$ \\
\hline 1.5 Plaque 4-6 weeks & 12 & 950 & $\begin{array}{l}\text { Std. Mean Difference (IV, Random, } \\
95 \% \mathrm{CI} \text { ) }\end{array}$ & $-1.45[-1.90,-1.00]$ \\
\hline 1.5.1 CHX versus no rinse & 3 & 433 & $\begin{array}{l}\text { Std. Mean Difference (IV, Random, } \\
95 \% \mathrm{CI} \text { ) }\end{array}$ & $-1.43[-2.39,-0.47]$ \\
\hline $\begin{array}{l}\text { 1.5.2 CHX versus place- } \\
\text { bo/control rinse }\end{array}$ & 9 & 517 & $\begin{array}{l}\text { Std. Mean Difference (IV, Random, } \\
95 \% \mathrm{CI} \text { ) }\end{array}$ & $-1.48[-2.07,-0.89]$ \\
\hline 1.6 Plaque 4-6 weeks PI (0-3) & 4 & 223 & $\begin{array}{l}\text { Mean Difference (IV, Random, 95\% } \\
\mathrm{Cl} \text { ) }\end{array}$ & $-0.58[-0.78,-0.39]$ \\
\hline 1.6.1 CHX versus no rinse & 2 & 114 & $\begin{array}{l}\text { Mean Difference (IV, Random, 95\% } \\
\mathrm{Cl})\end{array}$ & $-0.59[-0.94,-0.24]$ \\
\hline $\begin{array}{l}\text { 1.6.2 CHX versus place- } \\
\text { bo/control rinse }\end{array}$ & 2 & 109 & $\begin{array}{l}\text { Mean Difference (IV, Random, 95\% } \\
\mathrm{Cl} \text { ) }\end{array}$ & $-0.50[-0.97,-0.04]$ \\
\hline $\begin{array}{l}\text { 1.7 Plaque } 4-6 \text { weeks TQH } \\
(0-5)\end{array}$ & 5 & 546 & $\begin{array}{l}\text { Mean Difference (IV, Random, 95\% } \\
\mathrm{Cl} \text { ) }\end{array}$ & $-0.78[-0.85,-0.70]$ \\
\hline 1.7.1 CHX versus no rinse & 1 & 319 & $\begin{array}{l}\text { Mean Difference (IV, Random, 95\% } \\
\mathrm{Cl} \text { ) }\end{array}$ & $-0.83[-1.00,-0.66]$ \\
\hline $\begin{array}{l}\text { 1.7. } 2 \mathrm{CHX} \text { versus place- } \\
\text { bo/control rinse }\end{array}$ & 4 & 227 & $\begin{array}{l}\text { Mean Difference (IV, Random, 95\% } \\
\mathrm{CI})\end{array}$ & $-0.76[-0.85,-0.68]$ \\
\hline 1.8 Plaque 6 months & 11 & 2075 & $\begin{array}{l}\text { Std. Mean Difference (IV, Random, } \\
95 \% \mathrm{CI} \text { ) }\end{array}$ & $-1.43[-1.76,-1.10]$ \\
\hline 1.8.1 CHX versus no rinse & 2 & 142 & $\begin{array}{l}\text { Std. Mean Difference (IV, Random, } \\
95 \% \mathrm{Cl} \text { ) }\end{array}$ & $-0.68[-1.35,-0.01]$ \\
\hline $\begin{array}{l}\text { 1.8.2 CHX versus place- } \\
\text { bo/control rinse }\end{array}$ & 9 & 1933 & $\begin{array}{l}\text { Std. Mean Difference (IV, Random, } \\
95 \% \mathrm{Cl} \text { ) }\end{array}$ & $-1.59[-1.89,-1.29]$ \\
\hline 1.9 Plaque 6 months PI (0-3) & 5 & 1108 & $\begin{array}{l}\text { Mean Difference (IV, Random, 95\% } \\
\mathrm{Cl} \text { ) }\end{array}$ & $-0.62[-1.12,-0.12]$ \\
\hline 1.9.1 CHX versus no rinse & 2 & 142 & $\begin{array}{l}\text { Mean Difference (IV, Random, 95\% } \\
\mathrm{Cl} \text { ) }\end{array}$ & $-0.30[-0.42,-0.18]$ \\
\hline $\begin{array}{l}\text { 1.9.2 CHX versus place- } \\
\text { bo/control rinse }\end{array}$ & 3 & 966 & $\begin{array}{l}\text { Mean Difference (IV, Random, 95\% } \\
\mathrm{Cl} \text { ) }\end{array}$ & $-0.86[-1.46,-0.25]$ \\
\hline $\begin{array}{l}\text { 1.10 Plaque } 6 \text { months TQH } \\
(0-5)\end{array}$ & 6 & 967 & $\begin{array}{l}\text { Mean Difference (IV, Random, 95\% } \\
\mathrm{CI})\end{array}$ & $-0.73[-0.88,-0.57]$ \\
\hline $\begin{array}{l}1.10 .1 \mathrm{CHX} \text { versus place- } \\
\text { bo/control rinse }\end{array}$ & 6 & 967 & $\begin{array}{l}\text { Mean Difference (IV, Random, 95\% } \\
\mathrm{Cl} \text { ) }\end{array}$ & $-0.73[-0.88,-0.57]$ \\
\hline
\end{tabular}




\begin{tabular}{|c|c|c|c|c|}
\hline Outcome or subgroup title & No. of studies & $\begin{array}{l}\text { No. of partici- } \\
\text { pants }\end{array}$ & Statistical method & Effect size \\
\hline 1.11 Calculus 4-6 weeks & 2 & & $\begin{array}{l}\text { Mean Difference (IV, Random, 95\% } \\
\mathrm{CI})\end{array}$ & Subtotals only \\
\hline $\begin{array}{l}1.11 .1 \mathrm{CHX} \text { versus place- } \\
\text { bo/control rinse }\end{array}$ & 2 & 102 & $\begin{array}{l}\text { Mean Difference (IV, Random, 95\% } \\
\mathrm{CI})\end{array}$ & $0.02[-0.09,0.14]$ \\
\hline 1.12 Calculus 7-12 weeks & 6 & 425 & $\begin{array}{l}\text { Std. Mean Difference (IV, Random, } \\
95 \% \mathrm{Cl})\end{array}$ & $0.32[-0.04,0.69]$ \\
\hline 1.12.1 CHX versus no rinse & 1 & 95 & $\begin{array}{l}\text { Std. Mean Difference (IV, Random, } \\
95 \% \mathrm{Cl})\end{array}$ & $1.02[0.59,1.45]$ \\
\hline $\begin{array}{l}1.12 .2 \mathrm{CHX} \text { versus place- } \\
\text { bo/control rinse }\end{array}$ & 5 & 330 & $\begin{array}{l}\text { Std. Mean Difference (IV, Random, } \\
95 \% \mathrm{Cl})\end{array}$ & $0.14[-0.08,0.36]$ \\
\hline 1.13 Calculus 6 months & 4 & 323 & $\begin{array}{l}\text { Std. Mean Difference (IV, Random, } \\
95 \% \mathrm{Cl})\end{array}$ & $0.80[0.33,1.26]$ \\
\hline 1.13.1 CHX versus no rinse & 1 & 91 & $\begin{array}{l}\text { Std. Mean Difference (IV, Random, } \\
95 \% \mathrm{Cl})\end{array}$ & $1.39[0.93,1.85]$ \\
\hline $\begin{array}{l}1.13 .2 \mathrm{CHX} \text { versus place- } \\
\text { bo/control rinse }\end{array}$ & 3 & 232 & $\begin{array}{l}\text { Std. Mean Difference (IV, Random, } \\
95 \% \mathrm{Cl})\end{array}$ & $0.60[0.24,0.96]$ \\
\hline $\begin{array}{l}\text { 1.14 Tooth staining } 4-6 \text { weeks } \\
\text { dichotomous }\end{array}$ & 2 & 156 & Risk Ratio (M-H, Random, 95\% Cl) & $5.41[2.03,14.47]$ \\
\hline 1.14.1 CHX versus no rinse & 1 & 118 & Risk Ratio (M-H, Random, 95\% Cl) & $4.44[1.43,13.80]$ \\
\hline $\begin{array}{l}1.14 .2 \mathrm{CHX} \text { versus place- } \\
\text { bo/control rinse }\end{array}$ & 1 & 38 & Risk Ratio (M-H, Random, 95\% Cl) & $9.88[1.37,71.44]$ \\
\hline $\begin{array}{l}1.15 \text { Tooth staining } 7-12 \\
\text { weeks dichotomous }\end{array}$ & 1 & & Risk Ratio (M-H, Random, 95\% Cl) & Subtotals only \\
\hline 1.15.1 CHX versus no rinse & 1 & 118 & Risk Ratio (M-H, Random, 95\% Cl) & $2.50[1.29,4.83]$ \\
\hline 1.16 Tooth staining 4-6 weeks & 8 & 415 & $\begin{array}{l}\text { Std. Mean Difference (IV, Random, } \\
95 \% \mathrm{Cl})\end{array}$ & $1.07[0.80,1.34]$ \\
\hline 1.16.1 CHX versus no rinse & 1 & 94 & $\begin{array}{l}\text { Std. Mean Difference (IV, Random, } \\
95 \% \mathrm{Cl})\end{array}$ & $1.54[1.08,2.00]$ \\
\hline $\begin{array}{l}\text { 1.16.2 CHX versus place- } \\
\text { bo/control rinse }\end{array}$ & 7 & 321 & $\begin{array}{l}\text { Std. Mean Difference (IV, Random, } \\
95 \% \mathrm{Cl})\end{array}$ & $0.97[0.73,1.22]$ \\
\hline $\begin{array}{l}\text { 1.17 Tooth staining } 7-12 \\
\text { weeks }\end{array}$ & 11 & 581 & $\begin{array}{l}\text { Std. Mean Difference (IV, Random, } \\
95 \% \mathrm{Cl})\end{array}$ & $1.19[0.98,1.40]$ \\
\hline 1.17.1 CHX versus no rinse & 1 & 95 & $\begin{array}{l}\text { Std. Mean Difference (IV, Random, } \\
95 \% \mathrm{Cl})\end{array}$ & $1.32[0.88,1.77]$ \\
\hline $\begin{array}{l}1.17 .2 \mathrm{CHX} \text { versus place- } \\
\text { bo/control rinse }\end{array}$ & 10 & 486 & $\begin{array}{l}\text { Std. Mean Difference (IV, Random, } \\
95 \% \mathrm{Cl})\end{array}$ & $1.17[0.93,1.41]$ \\
\hline
\end{tabular}




\begin{tabular}{lllll}
\hline Outcome or subgroup title & No. of studies & $\begin{array}{l}\text { No. of partici- } \\
\text { pants }\end{array}$ & Statistical method & Effect size \\
\hline 1.18 Tooth staining 6 months & 4 & 323 & $\begin{array}{l}\text { Std. Mean Difference (IV, Random, } \\
95 \% \mathrm{Cl} \text { ) }\end{array}$ & $1.54[1.22,1.86]$ \\
\hline $1.18 .1 \mathrm{CHX}$ versus no rinse & 1 & 91 & $\begin{array}{l}\text { Std. Mean Difference (IV, Random, } \\
95 \% \mathrm{Cl})\end{array}$ & $1.18[0.73,1.62]$ \\
\hline $\begin{array}{l}1.18 .2 \mathrm{CHX} \text { versus place- } \\
\text { bo/control rinse }\end{array}$ & 3 & 232 & $\begin{array}{l}\text { Std. Mean Difference (IV, Random, } \\
95 \% \mathrm{Cl})\end{array}$ & $1.69[1.38,1.99]$ \\
\hline
\end{tabular}

\begin{tabular}{|c|c|c|c|c|c|c|c|c|c|c|}
\hline Study or Subgroup & Mean & $\begin{array}{l}\text { CHX } \\
\text { SD }\end{array}$ & Total & Mean & \multicolumn{2}{|l|}{ Control } & Weight & $\begin{array}{c}\text { Mean Difference } \\
\text { IV, Random, 95\% CI }\end{array}$ & \multicolumn{2}{|c|}{$\begin{array}{c}\text { Mean Difference } \\
\text { IV, Random, 95\% CI }\end{array}$} \\
\hline \multicolumn{11}{|c|}{ 1.1.1 CHX versus no rinse } \\
\hline Jose 2015 & 1.1149 & 0.1137 & 214 & 1.19 & 0.1435 & 105 & $20.3 \%$ & $-0.08[-0.11,-0.04]$ & $=$ & \\
\hline Rahmani 2006 & 1.12 & 0.31 & 10 & 1.4 & 0.31 & 10 & $8.1 \%$ & $-0.28[-0.55,-0.01]$ & & \\
\hline Subtotal $(95 \% \mathrm{CI})$ & & & 224 & & & 115 & $28.3 \%$ & $-0.13[-0.31,0.05]$ & & \\
\hline \multicolumn{11}{|c|}{ Heterogeneity: $\mathrm{Tau}^{2}=0.01 ; \mathrm{Chi}^{2}=2.16, \mathrm{df}=1(\mathrm{P}=0.14) ; \mathrm{I}^{2}=54 \%$} \\
\hline \multicolumn{11}{|c|}{ Test for overall effect: $\mathrm{Z}=1.44(\mathrm{P}=0.15)$} \\
\hline \multicolumn{11}{|c|}{ 1.1.2 CHX versus placebo/control rinse } \\
\hline Axelsson 1987 & 0.6271 & 0.297 & 42 & 1 & 0.3 & 22 & $13.7 \%$ & $-0.37[-0.53,-0.22]$ & $\longrightarrow$ & \\
\hline Bhat 2014 & 0.42 & 0.43 & 22 & 1.02 & 0.79 & 22 & $5.2 \%$ & $-0.60[-0.98,-0.22]$ & & \\
\hline Ernst 2005 & 0.45 & 0.45 & 33 & 0.56 & 0.49 & 28 & $9.4 \%$ & $-0.11[-0.35,0.13]$ & & _ \\
\hline Graziani 2015 & 0.6818 & 0.6659 & 55 & 0.8 & 0.7223 & 15 & $4.6 \%$ & $-0.12[-0.52,0.29]$ & & \\
\hline Jenkins 1993 & 0.54 & 0.27 & 49 & 0.72 & 0.26 & 50 & $16.8 \%$ & $-0.18[-0.28,-0.08]$ & - & \\
\hline Lopez-Jornet 2012 & 0.75 & 0.83 & 35 & 1.15 & 0.85 & 35 & $4.8 \%$ & $-0.40[-0.79,-0.01]$ & $\longleftarrow$ & \\
\hline Navarro 1998 & 0.048 & 0.099 & 9 & 0.174 & 0.2885 & 11 & $12.1 \%$ & $-0.13[-0.31,0.06]$ & & \\
\hline Sanz 1989 & 1.1164 & 0.6 & 17 & 1.244 & 0.6 & 21 & $5.0 \%$ & $-0.13[-0.51,0.26]$ & & \\
\hline Subtotal (95\% CI) & & & 262 & & & 204 & $71.7 \%$ & $-0.23[-0.34,-0.13]$ & & \\
\hline \multicolumn{11}{|c|}{ Heterogeneity: $\mathrm{Tau}^{2}=0.01 ; \mathrm{Chi}^{2}=11.37, \mathrm{df}=7(\mathrm{P}=0.12) ; \mathrm{I}^{2}=38 \%$} \\
\hline Total $(95 \%$ CI) & & & 486 & & & 319 & $100.0 \%$ & $-0.21[-0.31,-0.11]$ & & \\
\hline \multicolumn{11}{|c|}{ Heterogeneity: $\mathrm{Tau}^{2}=0.01 ; \mathrm{Chi}^{2}=27.51, \mathrm{df}=9(\mathrm{P}=0.001) ; \mathrm{I}^{2}=67 \%$} \\
\hline \multicolumn{9}{|c|}{ Test for overall effect: $\mathrm{Z}=4.11(\mathrm{P}<0.0001)$} & $-0.5-0.25$ & 0.25 \\
\hline Test for subgroup diffe & ices: $\mathrm{Chi}^{2}=$ & 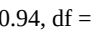 & $=0.3$ & 3), $\mathrm{I}^{2}=0 \%$ & & & & & CHX & \\
\hline
\end{tabular}




\section{Analysis 1.2. Comparison 1: $\mathrm{CHX}$ versus placebo/control mouthrinse} or no mouthrinse, Outcome 2: Gingival Index (0-3) 6 months

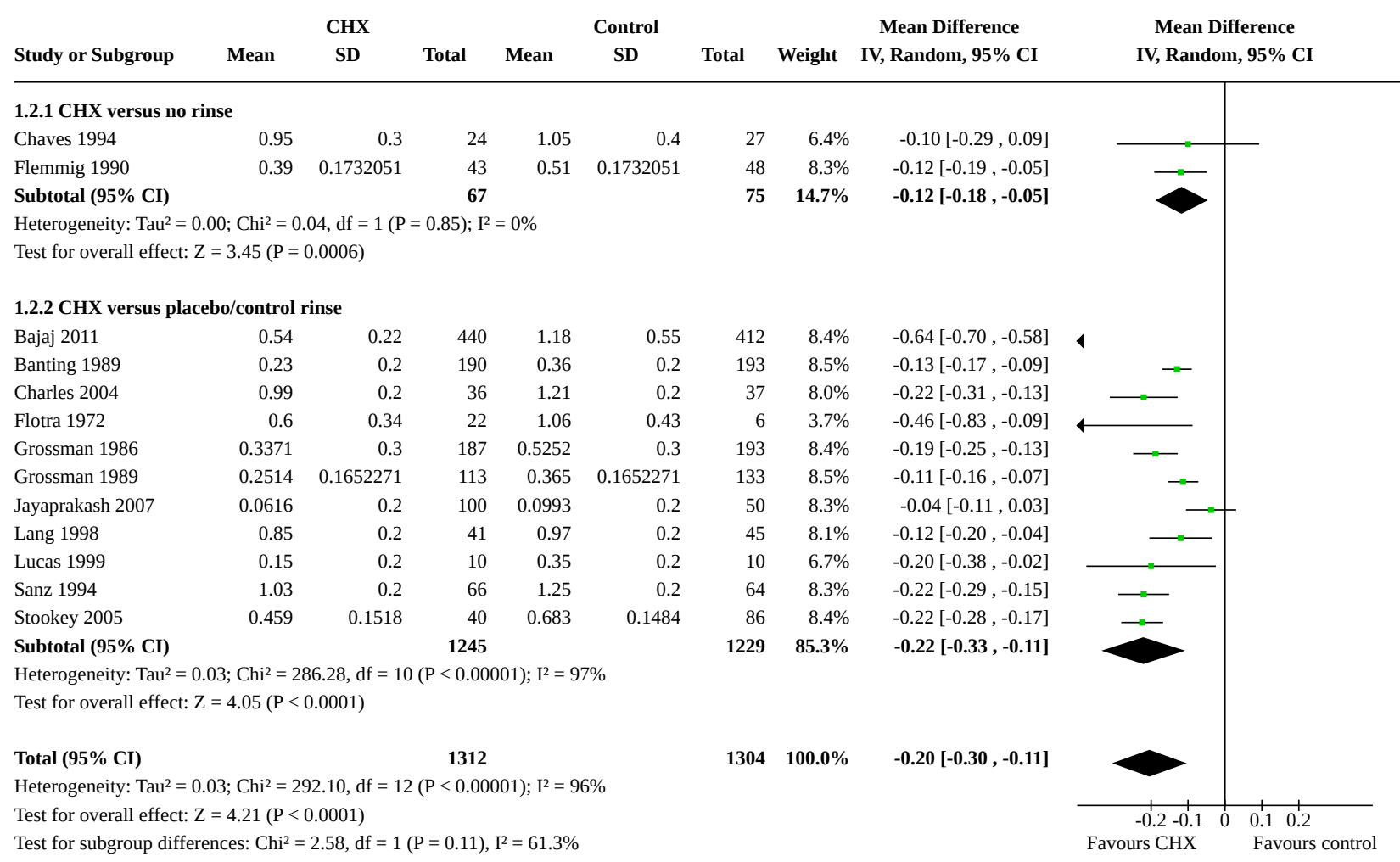

\section{Analysis 1.3. Comparison 1: $\mathrm{CHX}$ versus placebo/control mouthrinse or no mouthrinse, Outcome 3: Gingival bleeding 4-6 weeks}

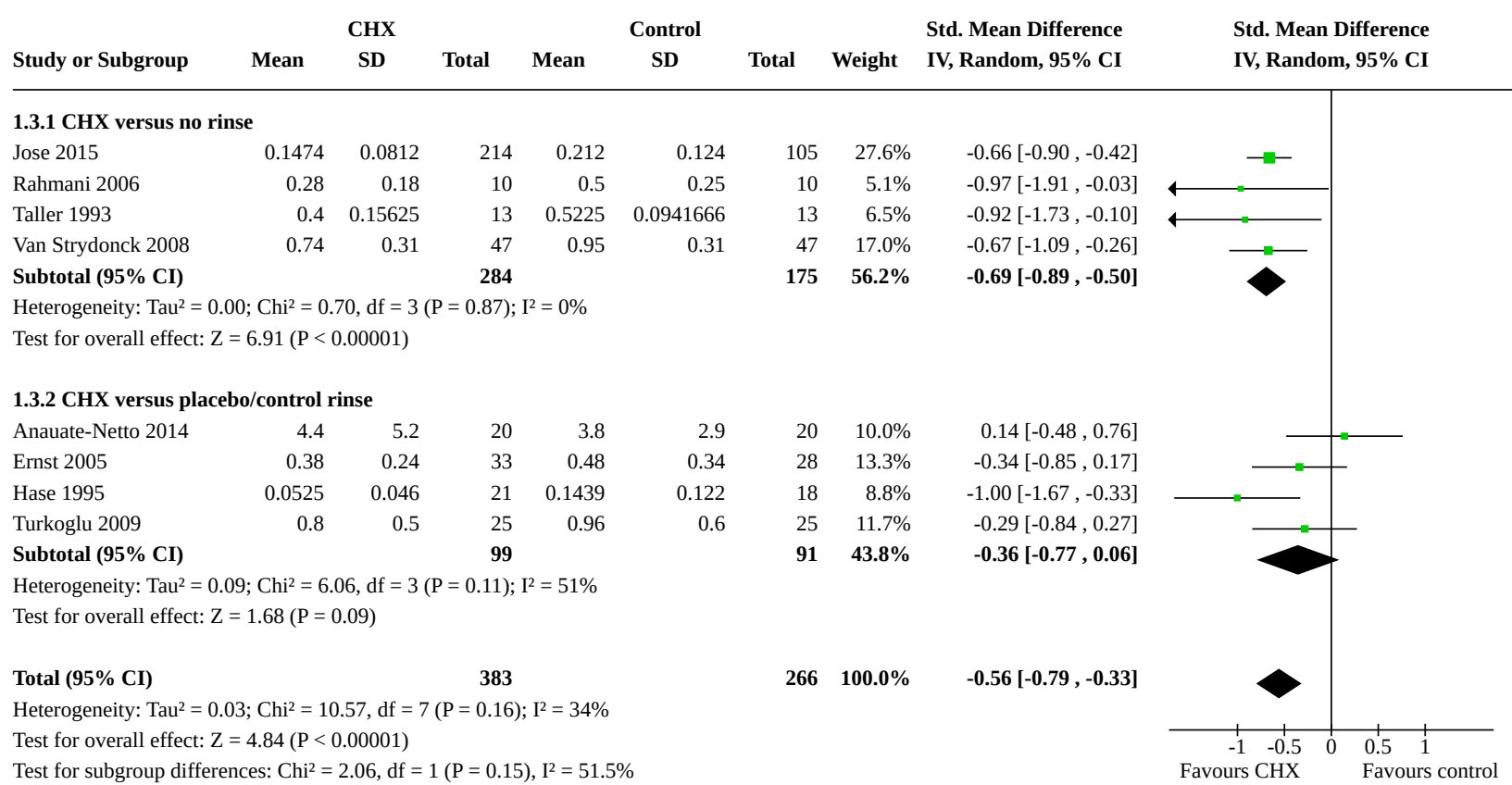




\title{
Analysis 1.4. Comparison 1: $\mathrm{CHX}$ versus placebo/control mouthrinse
} or no mouthrinse, Outcome 4: Gingival bleeding 6 months

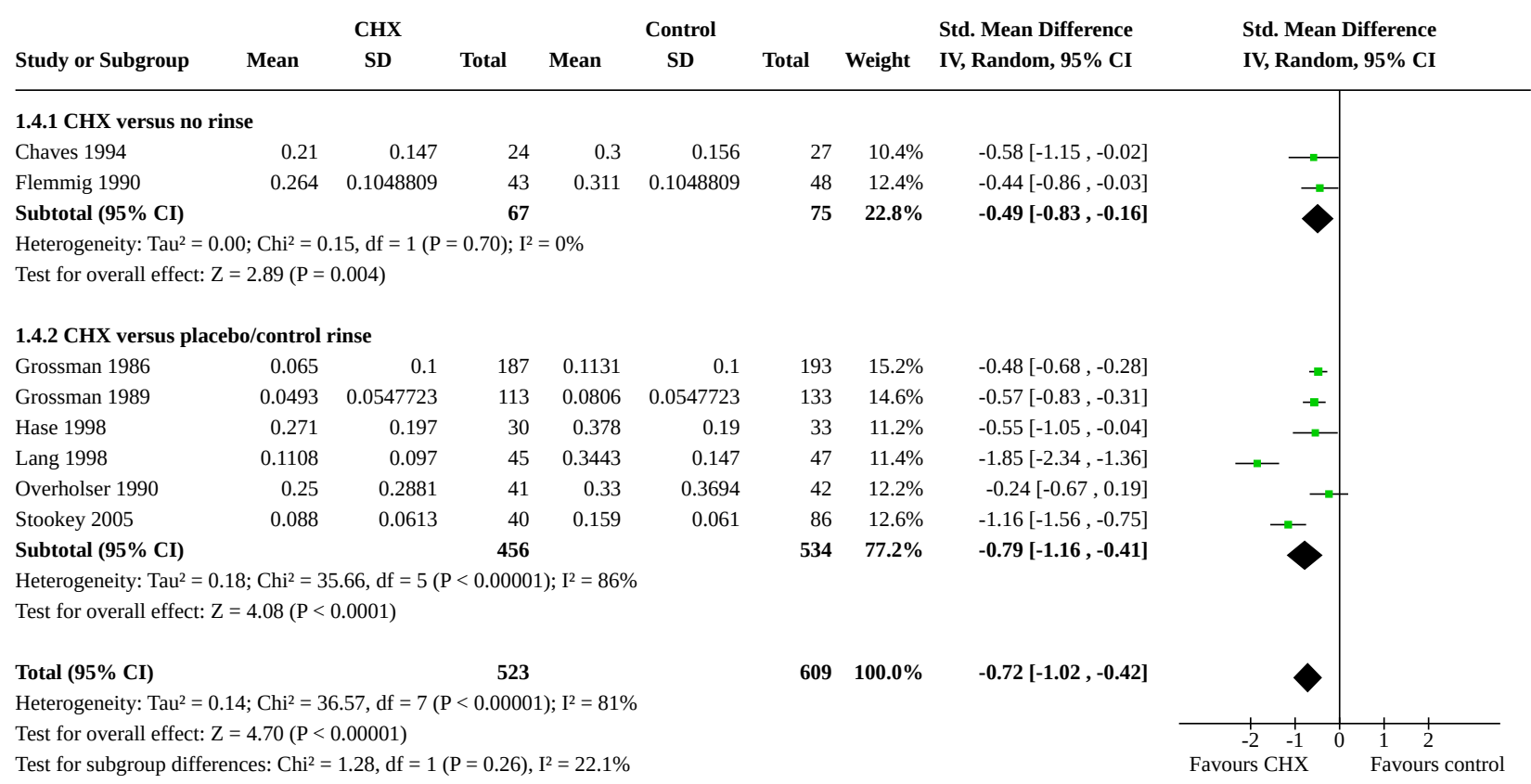

\begin{abstract}
Analysis 1.5. Comparison 1: CHX versus placebo/control mouthrinse or no mouthrinse, Outcome 5: Plaque 4-6 weeks
\end{abstract}

\begin{tabular}{|c|c|c|c|c|c|c|c|c|}
\hline \multirow[b]{2}{*}{ Study or Subgroup } & \multicolumn{3}{|c|}{ CHX } & \multicolumn{3}{|c|}{ Control } & \multicolumn{2}{|r|}{ Std. Mean Difference } \\
\hline & Mean & SD & Total & Mean & SD & Total & Weight & IV, Random, 95\% CI \\
\hline \multicolumn{9}{|c|}{ 1.5.1 CHX versus no rinse } \\
\hline Jose 2015 & 2.4694 & 0.8577 & 214 & 3.3 & 0.6353 & 105 & $9.9 \%$ & $-1.05[-1.29,-0.80]$ \\
\hline Rahmani 2006 & 0.52 & 0.48 & 10 & 0.88 & 0.39 & 10 & $7.2 \%$ & $-0.79[-1.71,0.13]$ \\
\hline Van Strydonck 2008 & 0.26 & 0.29 & 47 & 0.99 & 0.32 & 47 & $8.9 \%$ & $-2.37[-2.90,-1.84]$ \\
\hline Subtotal (95\% CI) & & & 271 & & & 162 & $26.0 \%$ & $-1.43[-2.39,-0.47]$ \\
\hline
\end{tabular}

Heterogeneity: $\mathrm{Tau}^{2}=0.63 ; \mathrm{Chi}^{2}=20.60, \mathrm{df}=2(\mathrm{P}<0.0001) ; \mathrm{I}^{2}=90 \%$

Test for overall effect: $\mathrm{Z}=2.91(\mathrm{P}=0.004)$

$\begin{array}{lrrrrrrrr}\text { 1.5.2 CHX versus placebo/control rinse } & & & & & & \\ \text { Axelsson 1987 } & 0.4143 & 0.4594 & 42 & 1.2 & 0.469 & 22 & 8.7 \% & -1.68[-2.27,-1.08] \\ \text { Bhat 2014 } & 0.68 & 0.14 & 22 & 1.46 & 0.19 & 22 & 6.1 \% & -4.59[-5.76,-3.42] \\ \text { Ernst 2005 } & 0.385 & 0.232 & 33 & 0.536 & 0.204 & 28 & 9.0 \% & -0.68[-1.20,-0.16] \\ \text { Graziani 2015 } & 0.1314 & 0.0842 & 55 & 0.214 & 0.0636 & 15 & 8.7 \% & -1.02[-1.61,-0.42] \\ \text { Hase 1995 } & 0.04 & 0.0458 & 21 & 0.75 & 0.4 & 18 & 7.4 \% & -2.55[-3.41,-1.68] \\ \text { Jenkins 1993 } & 1.15 & 0.76 & 49 & 1.8 & 0.51 & 50 & 9.4 \% & -1.00[-1.42,-0.58] \\ \text { Lopez-Jornet 2012 } & 0.83 & 0.84 & 35 & 1.06 & 0.85 & 35 & 9.2 \% & -0.27[-0.74,0.20] \\ \text { Navarro 1998 } & 1.706 & 0.783 & 9 & 2.291 & 0.4212 & 11 & 7.1 \% & -0.92[-1.86,0.02] \\ \text { Turkoglu 2009 } & 1.69 & 0.6 & 25 & 2.56 & 0.5 & 25 & 8.5 \% & -1.55[-2.19,-0.91] \\ \text { Subtotal (95\% CI) } & & & \mathbf{2 9 1} & & & \mathbf{2 2 6} & \mathbf{7 4 . 0 \%} & \mathbf{- 1 . 4 8 [ - 2 . 0 7 , - 0 . 8 9 ]}\end{array}$

Heterogeneity: $\mathrm{Tau}^{2}=0.69 ; \mathrm{Chi}^{2}=65.44, \mathrm{df}=8(\mathrm{P}<0.00001) ; \mathrm{I}^{2}=88 \%$

Test for overall effect: $\mathrm{Z}=4.90(\mathrm{P}<0.00001)$

Total (95\% CI)
Heterogeneity: Tau$^{2}=0.52 ;$ Chi $^{2}=86.66, d f=11$
Test for overall effect: $\mathrm{Z}=6.31(\mathrm{P}<0.00001) ; \mathrm{I}^{2}=87 \%$

Test for subgroup differences: $\mathrm{Chi}^{2}=0.01, \mathrm{df}=1(\mathrm{P}=0.93), \mathrm{I}^{2}=0 \%$
Std. Mean Difference

IV, Random, 95\% C 
Analysis 1.6. Comparison 1: $\mathrm{CHX}$ versus placebo/control mouthrinse or no mouthrinse, Outcome 6: Plaque 4-6 weeks PI (0-3)

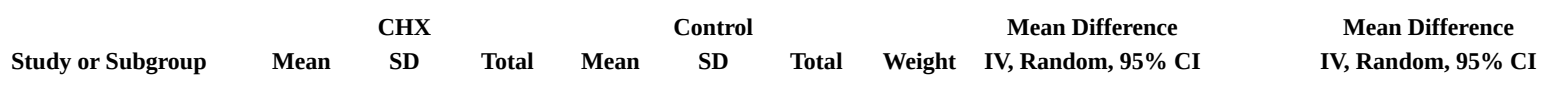

\subsubsection{CHX versus no rinse}

Rahmani 2006

Van Strydonck 2008

0.52

0.48

10

0.88

$\begin{array}{lll}0.39 & 10 & 16.4 \% \\ 0.32 & 47 & 36.8 \%\end{array}$

$-0.36[-0.74,0.02]$

Subtotal (95\% CI)

$\begin{array}{ll}0.26 & 0.29\end{array}$

47
57

0.99

$57 \quad 53.2 \%$

$-0.73[-0.85,-0.61]$

Heterogeneity: $\mathrm{Tau}^{2}=0.05 ; \mathrm{Chi}^{2}=3.24, \mathrm{df}=1(\mathrm{P}=0.07) ; \mathrm{I}^{2}=69 \%$

Test for overall effect: $\mathrm{Z}=3.30(\mathrm{P}=0.0010)$

1.6.2 CHX versus placebo/control rinse

\begin{tabular}{|c|c|c|c|c|c|c|c|c|}
\hline Hase 1995 & 0.04 & 0.0458 & 21 & 0.75 & 0.4 & 18 & $31.1 \%$ & $-0.71[-0.90,-0.52]$ \\
\hline Lopez-Jornet 2012 & 0.83 & 0.84 & 35 & 1.06 & 0.85 & 35 & $15.7 \%$ & $-0.23[-0.63,0.17]$ \\
\hline Subtotal (95\% CI) & & & 56 & & & 53 & $46.8 \%$ & $-0.50[-0.97,-0.04]$ \\
\hline
\end{tabular}

Heterogeneity: $\mathrm{Tau}^{2}=0.09 ; \mathrm{Chi}^{2}=4.63, \mathrm{df}=1(\mathrm{P}=0.03) ; \mathrm{I}^{2}=78 \%$

Test for overall effect: $\mathrm{Z}=2.12(\mathrm{P}=0.03)$

Total (95\% CI) 113

Heterogeneity: $\mathrm{Tau}^{2}=0.02 ; \mathrm{Chi}^{2}=8.34, \mathrm{df}=3(\mathrm{P}=0.04) ; \mathrm{I}^{2}=64 \%$

Test for overall effect: $\mathrm{Z}=5.82(\mathrm{P}<0.00001)$

Test for subgroup differences: $\mathrm{Chi}^{2}=0.09, \mathrm{df}=1(\mathrm{P}=0.77), \mathrm{I}^{2}=0 \%$

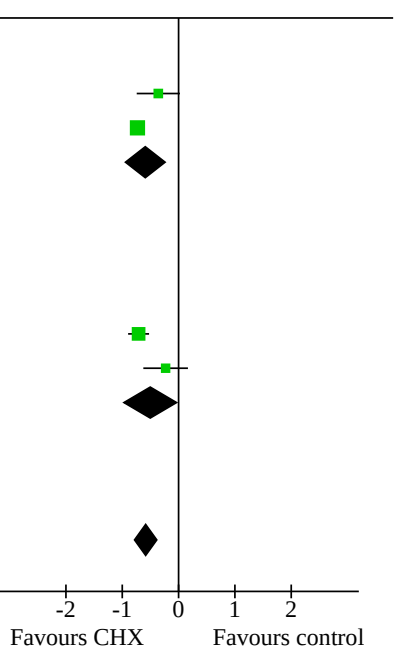

\section{Analysis 1.7. Comparison 1: CHX versus placebo/control mouthrinse or no mouthrinse, Outcome 7: Plaque 4-6 weeks TQH (0-5)}

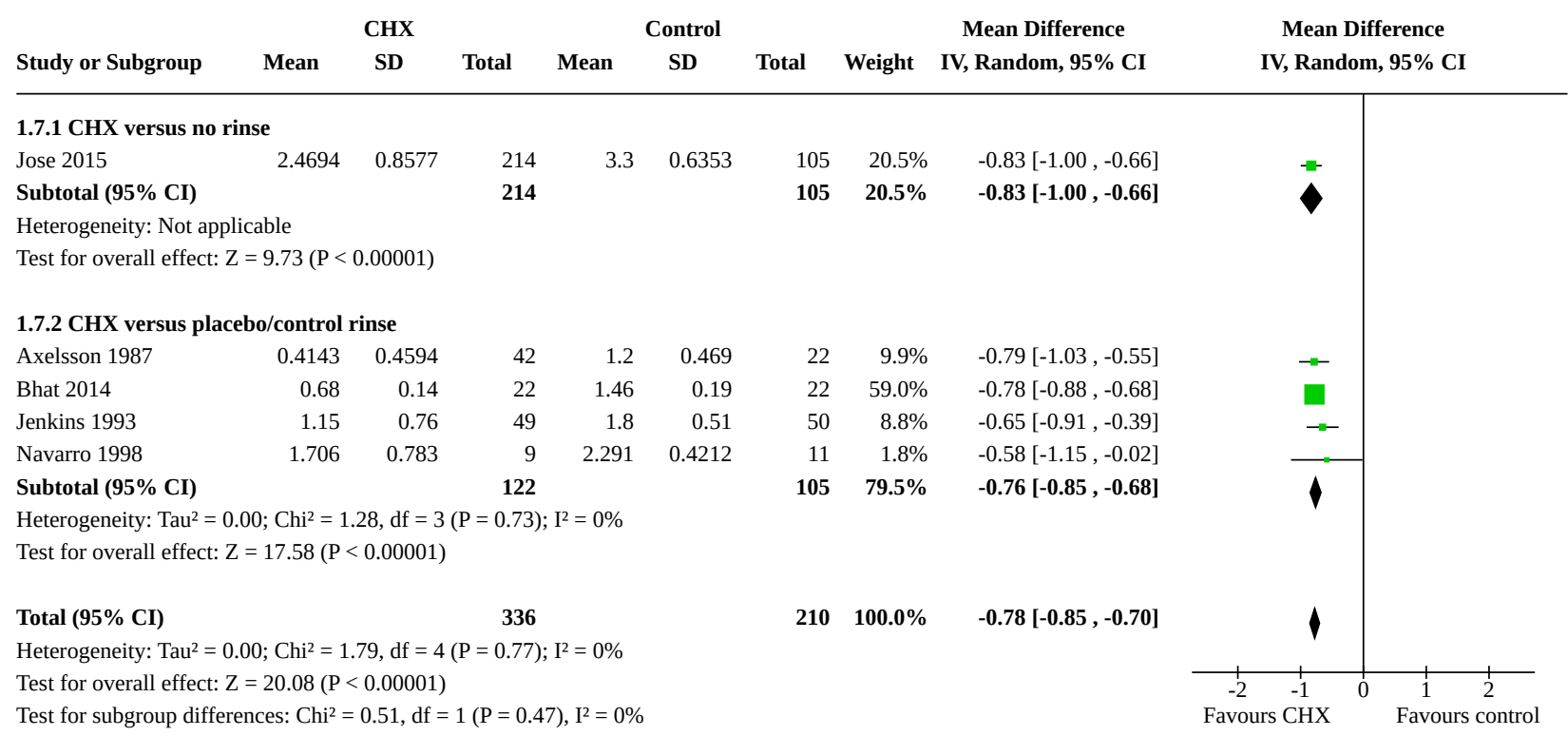


Analysis 1.8. Comparison 1: CHX versus placebo/control mouthrinse or no mouthrinse, Outcome 8: Plaque 6 months

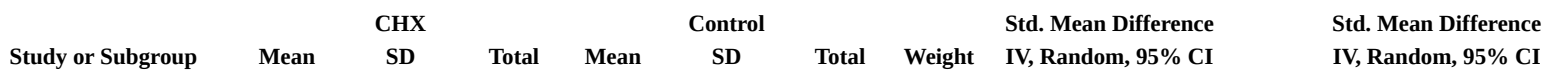

1.8.1 CHX versus no rinse

Chaves 1994

Flemmig 1990

$\begin{array}{ll}0.8 & 0.3\end{array}$

$0.43 \quad 0.3162278$

24

$\begin{array}{rrr}43 & 0.75 & 0.3162278\end{array}$

Subtotal (95\% CI)

67

Heterogeneity: $\mathrm{Tau}^{2}=0.17 ; \mathrm{Chi}^{2}=3.62, \mathrm{df}=1(\mathrm{P}=0.06) ; \mathrm{I}^{2}=72 \%$

Test for overall effect: $\mathrm{Z}=2.00(\mathrm{P}=0.05)$

1.8.2 CHX versus placebo/control rinse

Bajaj 2011

Charles 2004

Flotra 1972

Grossman 1986

Grossman 1989

Hase 1998

Lang 1998

Overholser 1990

Stookey 2005

Subtotal (95\% CI)

$\begin{array}{lr}0.72 & 0.26 \\ 1.71 & 0.5 \\ 0.15 & 0.17\end{array}$

0.26
0.5

440

$40-2.01$

$\begin{array}{lll}0.17 & 22 & 0.94\end{array}$

0.61

0.5

18

$188 \quad 1.56$

$0.76 \quad 0.5086256$

$0.96 \quad 0.6573$

$0.31-0.2561$

0.815

1.35

0.561
0.5

0.468

Heterogeneity: $\mathrm{Tau}^{2}=0.16 ; \mathrm{Chi}^{2}=49.48, \mathrm{df}=8(\mathrm{P}<0.00001) ; \mathrm{I}^{2}=84 \%$

Test for overall effect: $\mathrm{Z}=10.24(\mathrm{P}<0.00001)$

Total (95\% CI)

1018

Heterogeneity: $\mathrm{Tau}^{2}=0.25 ; \mathrm{Chi}^{2}=84.73, \mathrm{df}=10(\mathrm{P}<0.00001) ; \mathrm{I}^{2}=88 \%$

Test for overall effect: $\mathrm{Z}=8.47(\mathrm{P}<0.00001)$

Test for subgroup differences: $\mathrm{Chi}^{2}=5.85, \mathrm{df}=1(\mathrm{P}=0.02), \mathrm{I}^{2}=82.9 \%$
$-0.32[-0.87,0.23]$

$-1.00[-1.44,-0.57]$

$-0.68[-1.35,-0.01]$

95\% CI

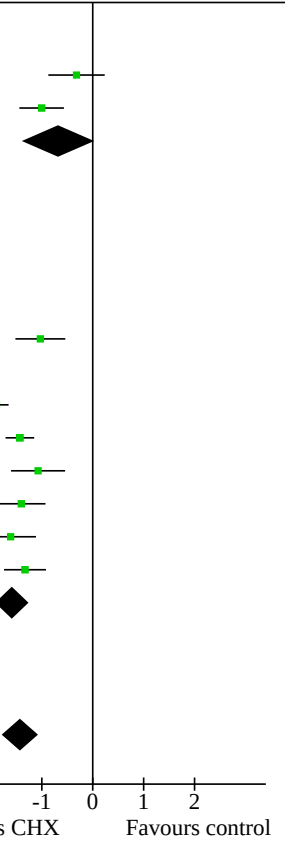

Analysis 1.9. Comparison 1: CHX versus placebo/control mouthrinse or no mouthrinse, Outcome 9: Plaque 6 months PI (0-3)

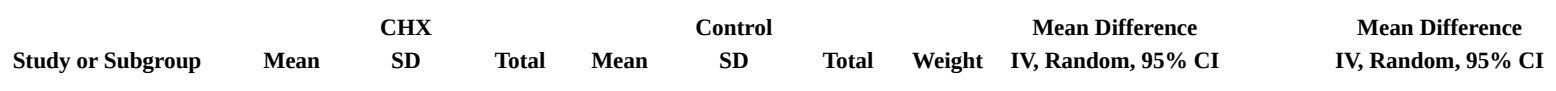

\subsubsection{CHX versus no rinse}

Chaves 1994

$0.43 \quad 0.3162278$

43

$\begin{array}{ll}0.75 & 0.3162278\end{array}$

$48 \quad 20.5 \%$

Subtotal (95\% CI)

0.8

$0.3 \quad 24$

67

$1 \quad 0.8$

$27 \quad 19.1 \%$

$75 \quad 39.7 \%$

- $0.45,-0.19]$

$-0.20[-0.52,0.12]$

$-0.30[-0.42,-0.18]$

Heterogeneity: $\mathrm{Tau}^{2}=0.00 ; \mathrm{Chi}^{2}=0.45, \mathrm{df}=1(\mathrm{P}=0.50) ; \mathrm{I}^{2}=0 \%$

Test for overall effect: $\mathrm{Z}=4.92(\mathrm{P}<0.00001)$

1.9.2 CHX versus placebo/control rinse

$\begin{array}{llrrrrrrr}\text { Bajaj 2011 } & 0.72 & 0.26 & 440 & 2.01 & 0.84 & 412 & 20.7 \% & -1.29[-1.37,-1.21] \\ \text { Flotra 1972 } & 0.15 & 0.17 & 22 & 0.94 & 0.4 & 6 & 19.1 \% & -0.79[-1.12,-0.46] \\ \text { Lang 1998 } & 0.31 & 0.2561 & 41 & 0.79 & 0.4 & 45 & 20.5 \% & -0.48[-0.62,-0.34] \\ \text { Subtotal (95\% CI) } & & & \mathbf{5 0 3} & & & \mathbf{4 6 3} & \mathbf{6 0 . 3 \%} & \mathbf{- 0 . 8 6}[-\mathbf{1 . 4 6 , - 0 . 2 5}]\end{array}$

Heterogeneity: $\mathrm{Tau}^{2}=0.28 ; \mathrm{Chi}^{2}=96.20, \mathrm{df}=2(\mathrm{P}<0.00001) ; \mathrm{I}^{2}=98 \%$

Test for overall effect: $\mathrm{Z}=2.76(\mathrm{P}=0.006)$

Total (95\% CI)

570

$538 \quad 100.0 \%$

$-0.62[-1.12,-0.12]$

Heterogeneity: Tau$^{2}=0.31 ; \mathrm{Chi}^{2}=209.21, \mathrm{df}=4(\mathrm{P}<0.00001) ; \mathrm{I}^{2}=98 \%$

Test for overall effect: $\mathrm{Z}=2.44(\mathrm{P}=0.01)$

Test for subgroup differences: $\mathrm{Chi}^{2}=3.06, \mathrm{df}=1(\mathrm{P}=0.08), \mathrm{I}^{2}=67.3 \%$

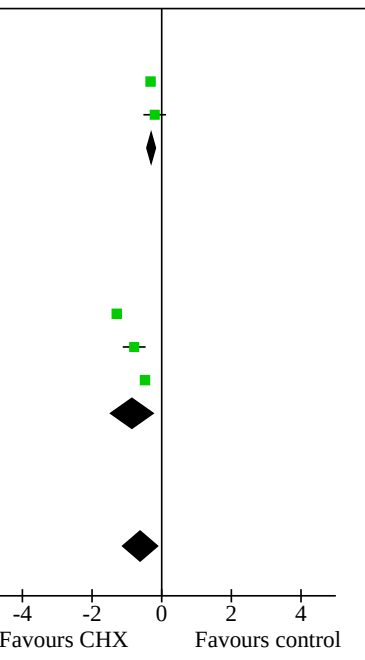




\section{Analysis 1.10. Comparison 1: $\mathrm{CHX}$ versus placebo/control mouthrinse} or no mouthrinse, Outcome 10: Plaque 6 months TQH (0-5)

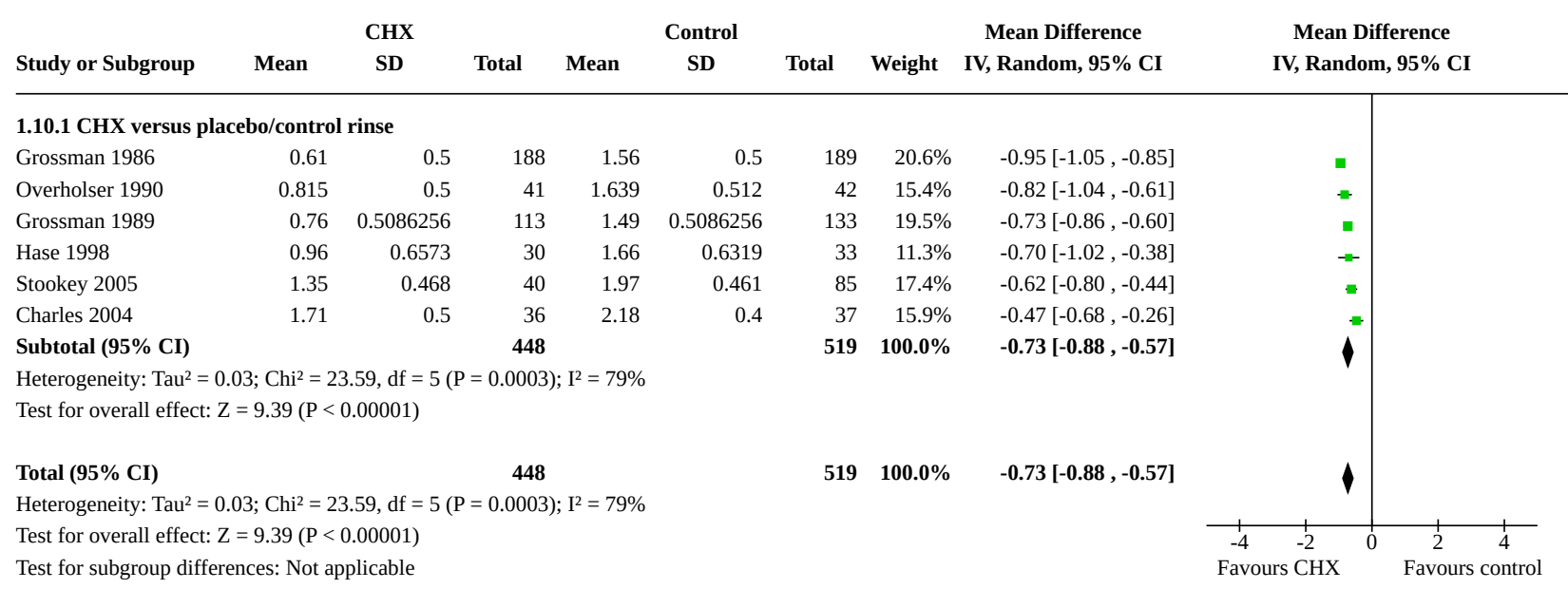

Analysis 1.11. Comparison 1: CHX versus placebo/control
mouthrinse or no mouthrinse, Outcome 11: Calculus 4-6 weeks

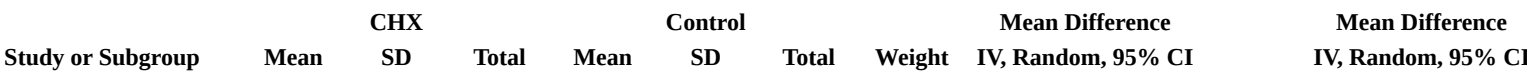

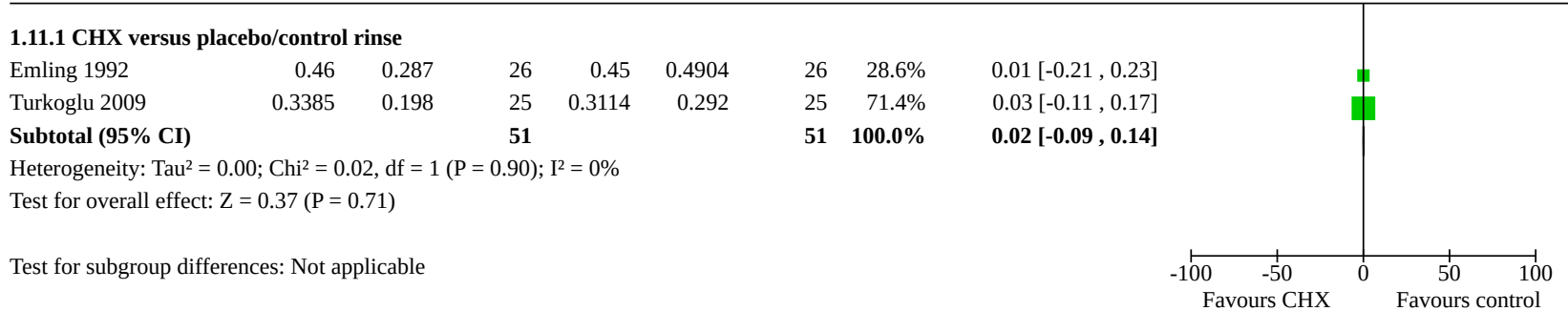


Analysis 1.12. Comparison 1: CHX versus placebo/control mouthrinse or no mouthrinse, Outcome 12: Calculus 7-12 weeks

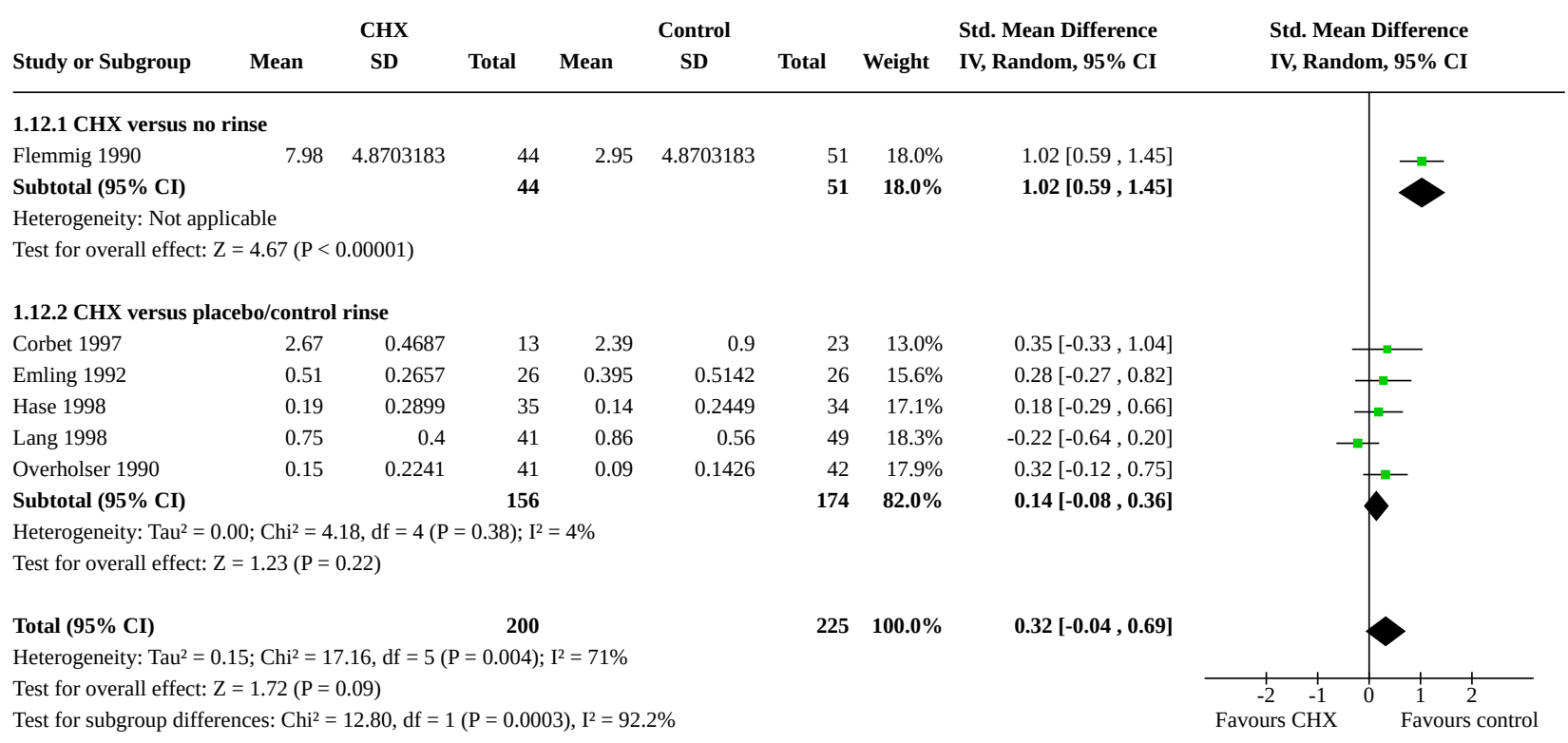

\section{Analysis 1.13. Comparison 1: CHX versus placebo/control mouthrinse or no mouthrinse, Outcome 13: Calculus 6 months}

\begin{tabular}{|c|c|c|c|c|c|c|c|c|c|}
\hline & & СHX & & & $\mathrm{H}$ alo & & & Std. Mean Difference & Std. Mean Difference \\
\hline Study or Subgroup & Mean & SD & Total & Mean & SD & Total & Weight & IV, Random, 95\% CI & IV, Random, 95\% CI \\
\hline
\end{tabular}

$\begin{array}{lllllllll}\text { 1.13.1 CHX versus no rinse } & & & & & & & & \\ \text { Flemmig } 1990 & 12.65 & 6.6257075 & 43 & 3.39 & 6.6257075 & 48 & 25.0 \% & 1.39[0.93,1.85] \\ \text { Subtotal (95\% CI) } & & & \mathbf{4 3} & & & \mathbf{4 8} & \mathbf{2 5 . 0 \%} & \mathbf{1 . 3 9}[\mathbf{0 . 9 3}, \mathbf{1 . 8 5}]\end{array}$

Heterogeneity: Not applicable

Test for overall effect: $\mathrm{Z}=5.90(\mathrm{P}<0.00001)$

1.13.2 CHX versus placebo/control rinse

$\begin{array}{lrlrlrrrr}\text { Hase 1998 } & 0.27 & 0.3122 & 30 & 0.14 & 0.2413 & 33 & 23.9 \% & 0.46[-0.04,0.96] \\ \text { Lang 1998 } & 0.97 & 0.4482 & 41 & 0.78 & 0.5367 & 45 & 25.9 \% & 0.38[-0.05,0.81] \\ \text { Overholser 1990 } & 0.36 & 0.3714 & 41 & 0.09 & 0.1361 & 42 & 25.1 \% & 0.96[0.51,1.42] \\ \text { Subtotal (95\% CI) } & & & \mathbf{1 1 2} & & & \mathbf{1 2 0} & \mathbf{7 5 . 0 \%} & \mathbf{0 . 6 0}[\mathbf{0 . 2 4}, \mathbf{0 . 9 6}]\end{array}$

Heterogeneity: $\mathrm{Tau}^{2}=0.05 ; \mathrm{Chi}^{2}=3.72, \mathrm{df}=2(\mathrm{P}=0.16) ; \mathrm{I}^{2}=46 \%$

Test for overall effect: $\mathrm{Z}=3.25(\mathrm{P}=0.001)$

Total (95\% CI)

155

Heterogeneity: $\mathrm{Tau}^{2}=0.17 ; \mathrm{Chi}^{2}=12.17, \mathrm{df}=3(\mathrm{P}=0.007) ; \mathrm{I}^{2}=75 \%$

Test for overall effect: $\mathrm{Z}=3.37(\mathrm{P}=0.0007)$

Test for subgroup differences: $\mathrm{Chi}^{2}=6.90, \mathrm{df}=1(\mathrm{P}=0.009), \mathrm{I}^{2}=85.5 \%$

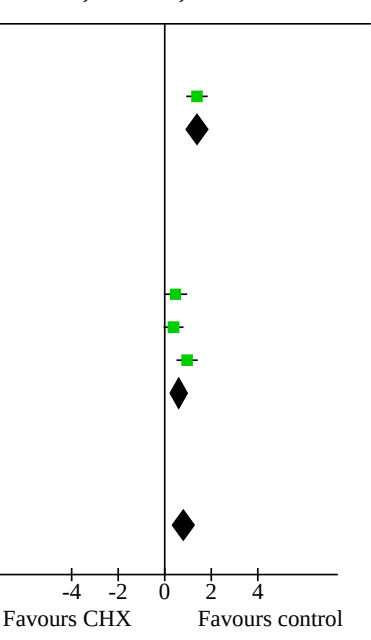


Analysis 1.14. Comparison 1: $\mathrm{CHX}$ versus placebo/control mouthrinse or no mouthrinse, Outcome 14: Tooth staining 4-6 weeks dichotomous

\begin{tabular}{|c|c|c|c|c|c|c|c|c|}
\hline \multirow[b]{2}{*}{ Study or Subgroup } & \multicolumn{2}{|c|}{ CHX } & \multicolumn{2}{|c|}{ Control } & \multirow[b]{2}{*}{ Weight } & \multirow{2}{*}{$\begin{array}{c}\text { Risk Ratio } \\
\text { M-H, Random, 95\% CI }\end{array}$} & \multirow{2}{*}{\multicolumn{2}{|c|}{$\begin{array}{c}\text { Risk Ratio } \\
\text { M-H, Random, 95\% CI }\end{array}$}} \\
\hline & Events & Total & Events & Total & & & & \\
\hline \multicolumn{9}{|c|}{ 1.14.1 CHX versus no rinse } \\
\hline Zimmer 2015 & 26 & 78 & 3 & 40 & $75.3 \%$ & $4.44[1.43,13.80]$ & & \\
\hline Subtotal (95\% CI) & & 78 & & 40 & $75.3 \%$ & $4.44[1.43,13.80]$ & & \\
\hline Total events: & 26 & & 3 & & & & & \\
\hline \multicolumn{9}{|c|}{ Heterogeneity: Not applicable } \\
\hline \multicolumn{9}{|c|}{ Test for overall effect: $\mathrm{Z}=2.58(\mathrm{P}=0.010)$} \\
\hline \multicolumn{9}{|c|}{ 1.14.2 CHX versus placebo/control rinse } \\
\hline Sanz 1989 & 8 & 17 & 1 & 21 & $24.7 \%$ & $9.88[1.37,71.44]$ & & \\
\hline Subtotal $(95 \%$ CI) & & 17 & & 21 & $24.7 \%$ & $9.88[1.37,71.44]$ & & \\
\hline Total events: & 8 & & 1 & & & & & \\
\hline \multicolumn{9}{|c|}{ Heterogeneity: Not applicable } \\
\hline \multicolumn{9}{|c|}{ Test for overall effect: $\mathrm{Z}=2.27(\mathrm{P}=0.02)$} \\
\hline Total (95\% CI) & & 95 & & 61 & $100.0 \%$ & $5.41[2.03,14.47]$ & & \\
\hline Total events: & 34 & & 4 & & & & & \\
\hline \multicolumn{5}{|c|}{ Heterogeneity: $\mathrm{Tau}^{2}=0.00 ; \mathrm{Chi}^{2}=0.47, \mathrm{df}=1(\mathrm{P}=0.49) ; \mathrm{I}^{2}=0 \%$} & & 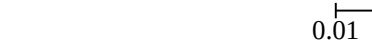 & 0.1 & 10 \\
\hline \multicolumn{5}{|c|}{ Test for overall effect: $\mathrm{Z}=3.37(\mathrm{P}=0.0008)$} & & & ours CHX & Favours \\
\hline
\end{tabular}

Analysis 1.15. Comparison 1: CHX versus placebo/control mouthrinse or no mouthrinse, Outcome 15: Tooth staining 7-12 weeks dichotomous

Control

Risk Ratio

Risk Ratio

Study or Subgroup

Events

Total
Total

Weight
M-H, Random, 95\% CI

1.15.1 CHX versus no rinse

Zimmer 2015

Subtotal (95\% CI)

$39 \quad 78$

8

$40 \quad 100.0 \%$

$2.50[1.29,4.83]$

Total events:

8

Heterogeneity: Not applicable

Test for overall effect: $\mathrm{Z}=2.73(\mathrm{P}=0.006)$

Test for subgroup differences: Not applicable

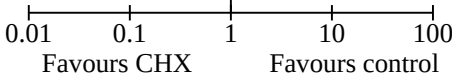


Analysis 1.16. Comparison 1: CHX versus placebo/control mouthrinse or no mouthrinse, Outcome 16: Tooth staining 4-6 weeks

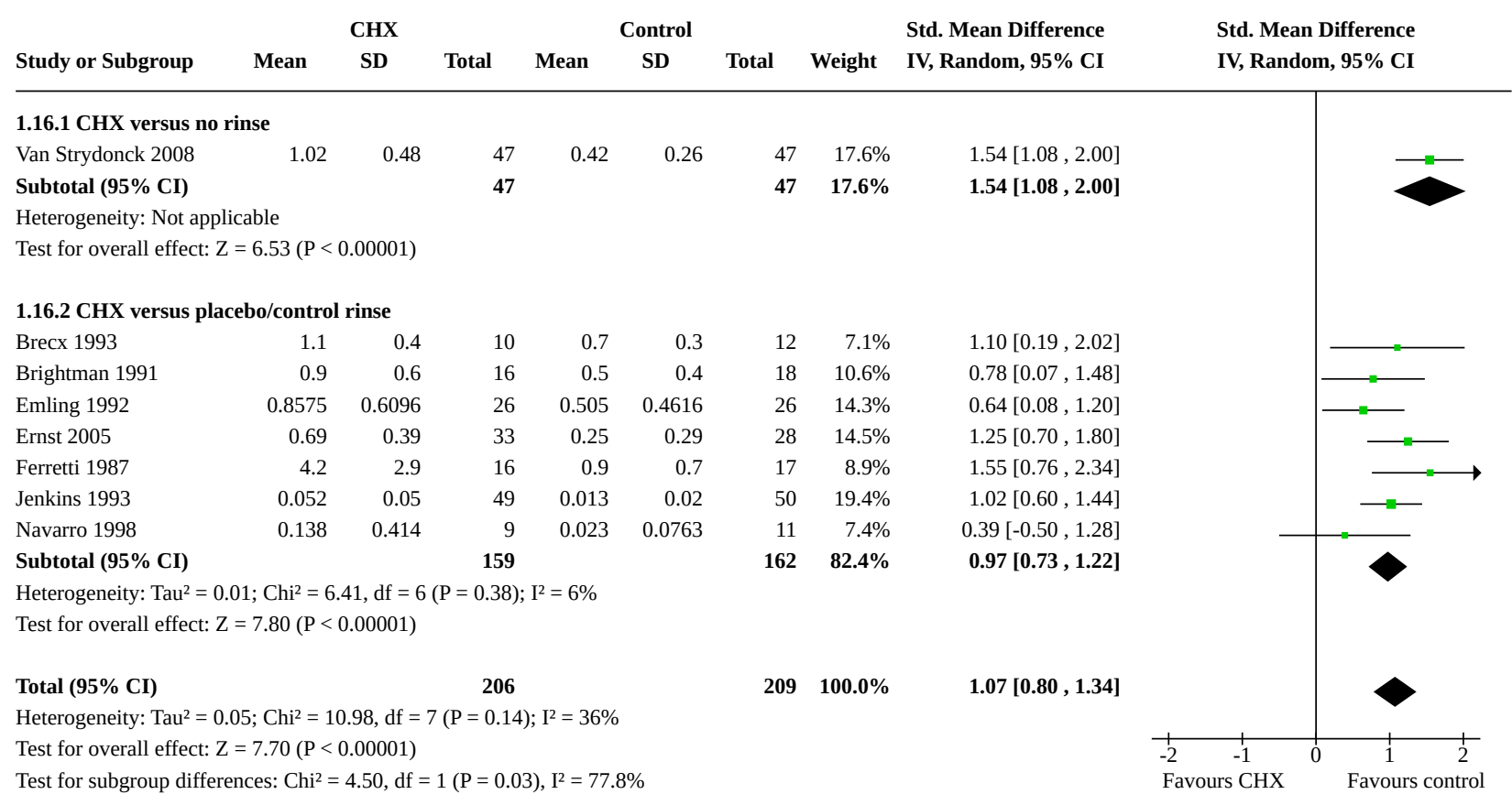

Analysis 1.17. Comparison 1: $\mathrm{CHX}$ versus placebo/control mouthrinse or no mouthrinse, Outcome 17: Tooth staining 7-12 weeks

\begin{tabular}{|c|c|c|c|c|c|c|c|c|c|}
\hline & & CHX & & & ontrol & & & Std. Mean Difference & Std. Mean Difference \\
\hline Study or Subgroup & Mean & SD & Total & Mean & SD & Total & Weight & IV, Random, 95\% CI & IV, Random, 95\% \\
\hline
\end{tabular}

\begin{tabular}{lllllllll}
\hline $\mathbf{1 . 1 7 . 1}$ CHX versus no rinse & & & & & & & & \\
Flemmig 1990 & 4.94 & 1.4696938 & 44 & 2.98 & 1.4696938 & 51 & $14.1 \%$ & $1.32[0.88,1.77]$ \\
Subtotal (95\% CI) & & & $\mathbf{4 4}$ & & & $\mathbf{5 1}$ & $\mathbf{1 4 . 1 \%}$ & $\mathbf{1 . 3 2}[\mathbf{0 . 8 8 , \mathbf { 1 . 7 7 }}$
\end{tabular}

Subtotal (95\% CI)

Heterogeneity: Not applicable

Test for overall effect: $\mathrm{Z}=5.80(\mathrm{P}<0.00001)$

1.17.2 CHX versus placebo/control rinse

$\begin{array}{lrrrrrrrr}\text { Anderson 1997 } & 1.465 & 0.6550572 & 13 & 0.565 & 0.5150728 & 15 & 5.2 \% & 1.50[0.64,2.35] \\ \text { Brecx 1993 } & 1.36 & 0.5 & 10 & 0.58 & 1 & 12 & 4.9 \% & 0.92[0.03,1.81] \\ \text { Brightman 1991 } & 1.4 & 0.7 & 16 & 0.5 & 0.4 & 18 & 6.1 \% & 1.57[0.79,2.35] \\ \text { Corbet 1997 } & 0.47 & 0.3245 & 13 & 0.31 & 0.1 & 23 & 7.2 \% & 0.75[0.04,1.45] \\ \text { Emling 1992 } & 0.92 & 0.5739 & 26 & 0.4425 & 0.4449 & 26 & 10.0 \% & 0.92[0.34,1.49] \\ \text { Ferretti 1987 } & 10.7 & 4.4 & 16 & 7.3 & 4.2 & 17 & 7.1 \% & 0.77[0.06,1.48] \\ \text { Hase 1998 } & 0.0813 & 0.0616 & 35 & 0.0167 & 0.0337 & 34 & 11.5 \% & 1.28[0.76,1.80] \\ \text { Joyston-Bechal 1993 } & 0.5 & 0.55 & 20 & 0.21 & 0.39 & 19 & 8.4 \% & 0.59[-0.05,1.24] \\ \text { Lang 1998 } & 1.16 & 0.6 & 41 & 0.39 & 0.35 & 49 & 12.9 \% & 1.59[1.11,2.07] \\ \text { Overholser 1990 } & 0.71 & 0.5955 & 41 & 0.08 & 0.1 & 42 & 12.6 \% & 1.47[0.98,1.96] \\ \text { Subtotal (95\% CI) } & & & \mathbf{2 3 1} & & & \mathbf{2 5 5} & \mathbf{8 5 . 9 \%} & \mathbf{1 . 1 7}[\mathbf{0 . 9 3}, \mathbf{1 . 4 1}]\end{array}$

Heterogeneity: $\mathrm{Tau}^{2}=0.04 ; \mathrm{Chi}^{2}=12.84, \mathrm{df}=9(\mathrm{P}=0.17) ; \mathrm{I}^{2}=30 \%$

Test for overall effect: $\mathrm{Z}=9.55(\mathrm{P}<0.00001)$

Total (95\% CI)

275

Heterogeneity: $\mathrm{Tau}^{2}=0.03 ; \mathrm{Chi}^{2}=13.13, \mathrm{df}=10(\mathrm{P}=0.22) ; \mathrm{I}^{2}=24 \%$

Test for overall effect: $\mathrm{Z}=11.13(\mathrm{P}<0.00001)$

Test for subgroup differences: Chi $^{2}=0.36, \mathrm{df}=1(\mathrm{P}=0.55), \mathrm{I}^{2}=0 \%$

$306 \quad 100.0 \% \quad 1.19[0.98,1.40]$ 
Analysis 1.18. Comparison 1: CHX versus placebo/control mouthrinse or no mouthrinse, Outcome 18: Tooth staining 6 months

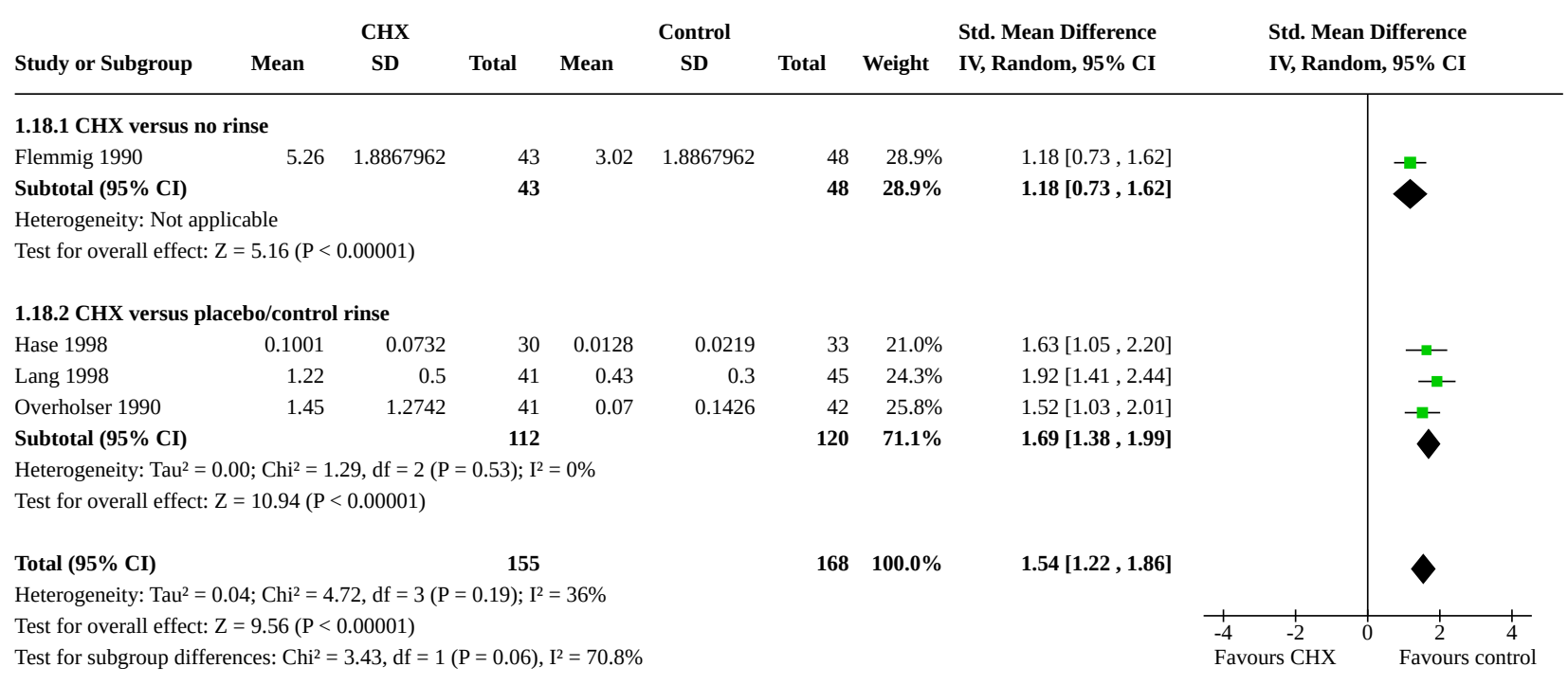

ADDITIONAL TABLES

Table 1. Random-effects metaregression analyses of Gingival Index (GI) at 4 to 6 weeks

\begin{tabular}{|c|c|c|c|c|c|}
\hline Characteristic & $\begin{array}{l}\text { Number of } \\
\text { studies }\end{array}$ & $\begin{array}{l}\text { Slope esti- } \\
\text { mate }\end{array}$ & $95 \% \mathrm{Cl}$ & Slope interpretation & P value \\
\hline Adults versus children & $\begin{array}{l}10 \text { - no studies } \\
\text { with just chil- } \\
\text { dren }\end{array}$ & & & & \\
\hline $\begin{array}{l}\text { Gingivitis alone versus } \\
\text { gingivitis with perio }\end{array}$ & 8 & 0.12 & -0.14 to 0.38 & $\begin{array}{l}\text { Increase in } \mathrm{Gl} \text { effect estimate for } \\
\text { gingivitis and perio }\end{array}$ & 0.30 \\
\hline Prophylaxis or not & 9 & 0.05 & -0.22 to 0.32 & $\begin{array}{l}\text { Increase in Gl effect estimate for } \\
\text { prophylaxis }\end{array}$ & 0.66 \\
\hline $\begin{array}{l}\text { Baseline gingivitis }<1 \\
\text { versus } \geq 1\end{array}$ & 9 & 0.02 & -0.25 to 0.30 & $\begin{array}{l}\text { Increase in GI effect estimate for } \\
\text { higher baseline score }\end{array}$ & 0.84 \\
\hline
\end{tabular}

$\mathrm{Cl}=$ confidence interval

Table 2. Random-effects metaregression analyses of Gingival Index (GI) at 6 months

\begin{tabular}{llllll}
\hline Characteristic & $\begin{array}{l}\text { Number of } \\
\text { studies }\end{array}$ & $\begin{array}{l}\text { Slope esti- } \\
\text { mate }\end{array}$ & $\mathbf{9 5 \%} \mathrm{Cl}$ & Slope interpretation & P value \\
\hline Adults versus children & 13 & -0.17 & -0.42 to 0.09 & $\begin{array}{l}\text { Increase in Gl effect estimate for } \\
\text { adults }\end{array}$ & 0.185 \\
\hline $\begin{array}{l}\text { Gingivitis alone versus } \\
\text { gingivitis with perio }\end{array}$ & 9 & 0.15 & -0.14 to 0.44 & $\begin{array}{l}\text { Increase in Gl effect estimate for } \\
\text { gingivitis and perio }\end{array}$ & 0.25 \\
\hline
\end{tabular}


Table 2. Random-effects metaregression analyses of Gingival Index (GI) at $\mathbf{6}$ months (Continued)

\begin{tabular}{llllll} 
Prophylaxis or not & 11 & -0.13 & -0.25 to -0.004 & $\begin{array}{l}\text { Increase in Gl effect estimate for } \\
\text { no prophylaxis }\end{array}$ & 0.045 \\
\hline $\begin{array}{l}\text { Baseline gingivitis } \\
<1 \text { versus } \geq 1\end{array}$ & 9 & -0.05 & -0.39 to 0.30 & $\begin{array}{l}\text { Decrease in Gl effect estimate for } \\
\text { higher baseline score }\end{array}$ & 0.75 \\
\hline
\end{tabular}

$\mathrm{Cl}=$ confidence interval.

Table 3. Chlorhexidine concentration: all trials

\begin{tabular}{|c|c|c|c|c|c|c|}
\hline $\begin{array}{l}\text { Outcome } \\
\text { (index) }\end{array}$ & Time & $\begin{array}{l}\text { Chlorhex- } \\
\text { idine con- } \\
\text { centration }\end{array}$ & $\begin{array}{l}\text { Studies } \\
\text { (partici- } \\
\text { pants) }\end{array}$ & MD/SMD 95\% Cl & Effect $P$ value & $\begin{array}{l}\text { Subgroup } \\
\text { P value }\end{array}$ \\
\hline \multirow{2}{*}{$\begin{array}{l}\text { Gingival } \\
\text { inflamma- } \\
\text { tion (Gingi- } \\
\text { val Index) }\end{array}$} & \multirow{2}{*}{$\begin{array}{l}4 \text { to } 6 \\
\text { weeks }\end{array}$} & 0.2 & $6(552)$ & MD $-0.27(-0.46$ to -0.09$)$ & 0.003 favours $\mathrm{CHX}$ & \multirow[t]{2}{*}{0.41} \\
\hline & & $\begin{array}{l}0.1 \text { and } \\
0.12\end{array}$ & $5(253)$ & MD $-0.19(-0.27$ to -0.10$)$ & $<0.0001$ favours $\mathrm{CHX}$ & \\
\hline \multirow{3}{*}{$\begin{array}{l}\text { Gingival } \\
\text { inflamma- } \\
\text { tion (Gingi- } \\
\text { val Index) }\end{array}$} & \multirow[t]{3}{*}{6 months } & 0.2 & $1(86)$ & MD $-0.12(-0.20$ to -0.04$)$ & 0.005 & \multirow{3}{*}{$\begin{array}{l}\text { Too few } \\
\text { studies in } \\
\text { subgroup }\end{array}$} \\
\hline & & $\begin{array}{l}0.1 \text { and } \\
0.12\end{array}$ & $10(2352)$ & MD $-0.22(-0.33$ to -0.11$)$ & $\begin{array}{l}<0.00001 \text { favours } \\
\mathrm{CHX}\end{array}$ & \\
\hline & & 0.05 & $1(150)$ & MD $-0.04(-0.11$ to 0.03$)$ & 0.28 & \\
\hline \multirow[t]{2}{*}{$\begin{array}{l}\text { Gingival } \\
\text { bleeding }\end{array}$} & \multirow[t]{2}{*}{$\begin{array}{l}4 \text { to } 6 \\
\text { weeks }\end{array}$} & 0.2 & $4(472)$ & SMD $-0.71(-0.90$ to -0.51$)$ & $\begin{array}{l}<0.00001 \text { favours } \\
\mathrm{CHX}\end{array}$ & \multirow[t]{2}{*}{0.18} \\
\hline & & $\begin{array}{l}0.1 \text { and } \\
0.12\end{array}$ & $3(127)$ & SMD $-0.32(-0.85$ to 0.21$)$ & 0.23 & \\
\hline \multirow{2}{*}{$\begin{array}{l}\text { Gingival } \\
\text { bleeding }\end{array}$} & \multirow[t]{2}{*}{6 months } & 0.2 & $2(155)$ & SMD $-1.20(-2.48$ to 0.08$)$ & 0.07 & \multirow[t]{2}{*}{0.34} \\
\hline & & 0.12 & $6(977)$ & SMD $-0.57(-0.79$ to -0.36$)$ & $\begin{array}{l}<0.00001 \text { favours } \\
\mathrm{CHX}\end{array}$ & \\
\hline \multirow[t]{2}{*}{ Plaque } & \multirow[t]{2}{*}{$\begin{array}{l}4 \text { to } 6 \\
\text { weeks }\end{array}$} & 0.2 & $8(685)$ & SMD $-1.75(-2.45$ to -1.04$)$ & $\begin{array}{l}<0.00001 \text { favours } \\
\mathrm{CHX}\end{array}$ & \multirow[t]{2}{*}{0.04} \\
\hline & & $\begin{array}{l}0.1 \text { and } \\
0.12\end{array}$ & $4(215)$ & SMD $-0.95(-1.23$ to -0.66$)$ & $\begin{array}{l}<0.00001 \text { favours } \\
\mathrm{CHX}\end{array}$ & \\
\hline \multirow[t]{2}{*}{ Plaque } & \multirow[t]{2}{*}{6 months } & 0.2 & $2(149)$ & SMD $-1.26(-1.61$ to -0.90$)$ & $\begin{array}{l}<0.00001 \text { favours } \\
\text { Chx }\end{array}$ & \multirow[t]{2}{*}{0.65} \\
\hline & & $\begin{array}{l}0.1 \text { and } \\
0.12\end{array}$ & $8(1898)$ & SMD $-1.38(-1.75$ to -1.00$)$ & $\begin{array}{l}<0.00001 \text { favours } \\
\mathrm{CHX}\end{array}$ & \\
\hline Calculus & $\begin{array}{l}4 \text { to } 6 \\
\text { weeks }\end{array}$ & 0.12 & $1(52)$ & MD 0.01 (-0.21 to 0.23$)$ & 0.93 & \\
\hline \multirow[t]{2}{*}{ Calculus } & \multirow{2}{*}{$\begin{array}{l}7 \text { to } 12 \\
\text { weeks }\end{array}$} & 0.2 & $2(159)$ & SMD $-0.03(-0.43$ to 0.36$)$ & 0.86 & \multirow[t]{2}{*}{0.05} \\
\hline & & 0.12 & $4(266)$ & SMD 0.52 (0.13 to 0.91$)$ & 0.10 & \\
\hline
\end{tabular}


Table 3. Chlorhexidine concentration: all trials (Continued)

\begin{tabular}{|c|c|c|c|c|c|c|}
\hline \multirow[t]{2}{*}{ Calculus } & \multirow[t]{2}{*}{6 months } & 0.2 & $2(149)$ & SMD 0.41 (0.09 to 0.74$)$ & 0.01 & \multirow[t]{2}{*}{0.005} \\
\hline & & 0.12 & $2(174)$ & SMD 1.17 (0.76 to 1.59$)$ & $\begin{array}{l}<0.00001 \text { favours } \\
\text { control }\end{array}$ & \\
\hline \multirow[t]{2}{*}{$\begin{array}{l}\text { Tooth } \\
\text { staining }\end{array}$} & \multirow[t]{2}{*}{$\begin{array}{l}4 \text { to } 6 \\
\text { weeks }\end{array}$} & 0.2 & $2(116)$ & SMD 1.45 (1.04 to 1.87$)$ & $\begin{array}{l}<0.00001 \text { favours } \\
\text { control }\end{array}$ & \multirow[t]{2}{*}{0.05} \\
\hline & & $\begin{array}{l}0.1 \text { and } \\
0.12\end{array}$ & $6(299)$ & SMD 0.96 (0.68 to 1.24 ) & $\begin{array}{l}<0.00001 \text { favours } \\
\text { control }\end{array}$ & \\
\hline \multirow[t]{3}{*}{$\begin{array}{l}\text { Tooth } \\
\text { staining }\end{array}$} & \multirow[t]{3}{*}{$\begin{array}{l}7 \text { to } 12 \\
\text { weeks }\end{array}$} & 0.2 & $3(181)$ & SMD 1.38 (1.05 to 1.71$)$ & $\begin{array}{l}<0.00001 \text { favours } \\
\text { control }\end{array}$ & \multirow[t]{3}{*}{0.10} \\
\hline & & 0.12 & $7(361)$ & SMD 1.2 (0.96 to 1.45$)$ & $\begin{array}{l}<0.00001 \text { favours } \\
\text { control }\end{array}$ & \\
\hline & & 0.05 & $1(39)$ & SMD $0.59(-0.05$ to 1.24$)$ & 0.07 & \\
\hline \multirow[t]{2}{*}{$\begin{array}{l}\text { Tooth } \\
\text { staining }\end{array}$} & \multirow[t]{2}{*}{6 months } & 0.2 & $2(149)$ & SMD 1.79 (1.41 to 2.17 ) & $\begin{array}{l}<0.00001 \text { favours } \\
\text { control }\end{array}$ & \multirow[t]{2}{*}{0.08} \\
\hline & & 0.12 & $2(174)$ & SMD 1.33 (1.00 to 1.66$)$ & $\begin{array}{l}<0.00001 \text { favours } \\
\text { control }\end{array}$ & \\
\hline
\end{tabular}

$\mathrm{CHX}=$ chlorhexidine; $\mathrm{Cl}=$ confidence interval; $\mathrm{MD}=$ mean difference; $\mathrm{SMD}=$ standardised mean difference.

Studies where the concentration of the chlorhexidine mouthrinse was not reported (Turkoglu 2009) and where data relate to a combination of different chlorhexidine concentrations (Flotra 1972) were excluded from this analysis. Therefore, in certain analyses, the number of studies and participants presented in the table are different from the data presented in the main analysis.

Table 4. Chlorhexidine rinse frequency of use: all trials

\begin{tabular}{|c|c|c|c|c|c|c|}
\hline $\begin{array}{l}\text { Outcome } \\
\text { (index) }\end{array}$ & Time & $\begin{array}{l}\text { Chlorhexidine } \\
\text { frequency }\end{array}$ & $\begin{array}{l}\text { Studies } \\
\text { (partici- } \\
\text { pants) }\end{array}$ & MD/SMD 95\% Cl & $\begin{array}{l}\text { Effect } P \\
\text { value }\end{array}$ & $\begin{array}{l}\text { Subgroup } \\
\text { P value }\end{array}$ \\
\hline \multirow{2}{*}{$\begin{array}{l}\text { Gingival in- } \\
\text { flammation } \\
\text { (Gingival In- } \\
\text { dex) }\end{array}$} & \multirow{2}{*}{$\begin{array}{l}4 \text { to } 6 \\
\text { weeks }\end{array}$} & Twice per day & $9(785)$ & MD $-0.22(-0.33$ to -0.11$)$ & $<0.0001$ & \multirow{2}{*}{$\begin{array}{l}\text { Too few } \\
\text { studies in } \\
\text { subgroup }\end{array}$} \\
\hline & & Once per day & $1(20)$ & MD $-0.13(-0.31$ to 0.06$)$ & 0.18 & \\
\hline \multirow{2}{*}{$\begin{array}{l}\text { Gingival in- } \\
\text { flammation } \\
\text { (Gingival In- } \\
\text { dex) }\end{array}$} & \multirow[t]{2}{*}{6 months } & Twice per day & $11(1614)$ & MD $-0.17(-0.20$ to -0.13$)$ & $<0.0001$ & \multirow[t]{2}{*}{0.56} \\
\hline & & Once per day & $2(1002)$ & MD $-0.34(-0.93$ to 0.25$)$ & 0.26 & \\
\hline $\begin{array}{l}\text { Gingival } \\
\text { bleeding }\end{array}$ & $\begin{array}{l}4 \text { to } 6 \\
\text { weeks }\end{array}$ & Twice per day & $8(649)$ & SMD $-0.56(-0.79$ to -0.33$)$ & $<0.0001$ & - \\
\hline $\begin{array}{l}\text { Gingival } \\
\text { bleeding }\end{array}$ & 6 months & Twice per day & $8(1132)$ & SMD $-0.72(-1.02$ to -0.42$)$ & $<0.0001$ & - \\
\hline \multirow[t]{2}{*}{ Plaque } & \multirow{2}{*}{$\begin{array}{l}4 \text { to } 6 \\
\text { weeks }\end{array}$} & Twice per day & $11(930)$ & SMD $-1.49(-1.97$ to -1.02$)$ & $<0.0001$ & \multirow{2}{*}{$\begin{array}{l}\text { Too few } \\
\text { studies in } \\
\text { subgroup }\end{array}$} \\
\hline & & Once per day & $1(20)$ & SMD -0.92 (-1.86 to 0.02$)$ & 0.05 & \\
\hline
\end{tabular}


Table 4. Chlorhexidine rinse frequency of use: all trials (Continued)

\begin{tabular}{|c|c|c|c|c|c|c|}
\hline \multirow[t]{2}{*}{ Plaque } & \multirow[t]{2}{*}{6 months } & Twice per day & $10(1223)$ & SMD $-1.34(-1.66$ to -1.03$)$ & $<0.0001$ & \multirow{2}{*}{$\begin{array}{l}\text { Too few } \\
\text { studies in } \\
\text { subgroup }\end{array}$} \\
\hline & & Once a day & $1(852)$ & SMD -2.10 (-2.27 to -1.93$)$ & $<0.0001$ & \\
\hline Calculus & $\begin{array}{l}4 \text { to } 6 \\
\text { weeks }\end{array}$ & Twice per day & $1(50)$ & MD $0.03(-0.11$ to 0.17$)$ & 0.70 & - \\
\hline Calculus & $\begin{array}{l}7 \text { to } 12 \\
\text { weeks }\end{array}$ & Twice per day & $5(373)$ & SMD $0.33(-0.11$ to 0.77$)$ & 0.14 & - \\
\hline Calculus & 6 months & Twice per day & $4(323)$ & SMD 0.80 (0.33 to 1.26$)$ & 0.0007 & - \\
\hline \multirow[t]{3}{*}{$\begin{array}{l}\text { Tooth stain- } \\
\text { ing }\end{array}$} & $\begin{array}{l}4 \text { to } 6 \\
\text { weeks }\end{array}$ & 3 times per day & $1(33)$ & SMD 1.55 (0.76 to 2.34 ) & $<0.0001$ & \multirow{3}{*}{$\begin{array}{l}\text { Too few } \\
\text { studies in } \\
\text { subgroup }\end{array}$} \\
\hline & & Twice per day & $5(310)$ & SMD 1.18 (0.93 to 1.44$)$ & $<0.0001$ & \\
\hline & & Once per day & $1(20)$ & SMD 0.39 (-0.5 to 1.28$)$ & 0.39 & \\
\hline \multirow{2}{*}{$\begin{array}{l}\text { Tooth stain- } \\
\text { ing }\end{array}$} & $\begin{array}{l}7 \text { to } 12 \\
\text { weeks }\end{array}$ & 3 times per day & $1(33)$ & SMD 0.77 (0.06 to 1.48 ) & 0.03 & \multirow{2}{*}{$\begin{array}{l}\text { Too few } \\
\text { studies in } \\
\text { subgroup }\end{array}$} \\
\hline & & Twice per day & $9(496)$ & SMD 1.26 (1.04 to 1.49$)$ & $<0.0001$ & \\
\hline $\begin{array}{l}\text { Tooth stain- } \\
\text { ing }\end{array}$ & 6 months & Twice per day & $4(323)$ & SMD 1.54 (1.22 to 1.86$)$ & $<0.0001$ & - \\
\hline
\end{tabular}

$\mathrm{Cl}=$ confidence interval $\mathrm{MD}=$ mean difference $\mathrm{SMD}=$ standardised mean difference .

Table 5. Results for gingivitis and plaque at 7 to 12 weeks, $>6$ months and long term (after cessation of mouthrinsing)

\begin{tabular}{|c|c|c|c|c|c|c|}
\hline $\begin{array}{l}\text { Outcome (in- } \\
\text { dex) }\end{array}$ & Time & $\begin{array}{l}\text { Chlorhexi- } \\
\text { dine conc } \\
(\%)\end{array}$ & $\begin{array}{l}\text { Studies } \\
\text { (partici- } \\
\text { pants) }\end{array}$ & MD/SMD 95\% Cl & Effect $P$ value & Heterogeneity \\
\hline \multicolumn{7}{|c|}{ Results for studies with no rinse control arms } \\
\hline $\begin{array}{l}\text { Gingival bleed- } \\
\text { ing }\end{array}$ & $\begin{array}{l}7 \text { to } 12 \\
\text { weeks }\end{array}$ & $<0.1$ & $2(196)$ & MD $-0.07(-0.16$ to 0.02$)$ & 0.13 favours $\mathrm{CHX}$ & $P=0.19,12=40 \%$ \\
\hline Plaque & $\begin{array}{l}7 \text { to } 12 \\
\text { weeks }\end{array}$ & $<0.1$ & $2(196)$ & SMD $-0.77(-1.07$ to -0.47$)$ & $\begin{array}{l}<0.00001 \text { favours } \\
\mathrm{CHX}\end{array}$ & $P=0.61, I^{2}=0 \%$ \\
\hline \multicolumn{7}{|c|}{ Results for studies with placebo/control rinse arms } \\
\hline $\begin{array}{l}\text { Gingival inflam- } \\
\text { mation (Gingi- } \\
\text { val Index) }\end{array}$ & $\begin{array}{l}7 \text { to } 12 \\
\text { weeks }\end{array}$ & $\begin{array}{l}0.2 \text { and } \\
0.12\end{array}$ & $4(144)$ & MD $-0.47(-0.76$ to -0.18$)$ & 0.001 favours $\mathrm{CHX}$ & $\begin{array}{l}P<0.0001,\left.\right|^{2}= \\
86 \%\end{array}$ \\
\hline $\begin{array}{l}\text { Gingival inflam- } \\
\text { mation (Gingi- } \\
\text { val Index) }\end{array}$ & $>6$ months & $\begin{array}{l}0.1 \text { and } \\
0.12\end{array}$ & $2(1124)$ & MD $-0.50(-1.11$ to 0.11$)$ & 0.11 favours $\mathrm{CHX}$ & $\begin{array}{l}\mathrm{P}<0.0001,1^{2}= \\
99 \%\end{array}$ \\
\hline $\begin{array}{l}\text { Gingival bleed- } \\
\text { ing }\end{array}$ & $\begin{array}{l}7 \text { to } 12 \\
\text { weeks }\end{array}$ & $\begin{array}{l}0.12 \text { and }< \\
0.1\end{array}$ & $5(182)$ & SMD $-1.29(-1.85$ to -0.72$)$ & $\begin{array}{l}<0.00001 \text { favours } \\
\mathrm{CHX}\end{array}$ & $P=0.02, I^{2}=64 \%$ \\
\hline
\end{tabular}


Table 5. Results for gingivitis and plaque at 7 to 12 weeks, > 6 months and long term (after cessation of mouthrinsing) (Continued)

\begin{tabular}{|c|c|c|c|c|c|c|}
\hline $\begin{array}{l}\text { Gingival bleed- } \\
\text { ing }\end{array}$ & Long term & 0.12 & $3(99)$ & MD $-0.12(-0.2$ to -0.04$)$ & 0.003 favours $\mathrm{CHX}$ & $P=0.33,\left.\right|^{2}=11 \%$ \\
\hline Plaque & $\begin{array}{l}7 \text { to } 12 \\
\text { weeks }\end{array}$ & $\begin{array}{l}0.2,0.12 \\
\text { and }<0.1\end{array}$ & $10(423)$ & SMD $-1.74(-2.51$ to -0.98$)$ & $\begin{array}{l}<0.00001 \text { favours } \\
\mathrm{CHX}\end{array}$ & $\begin{array}{l}P<0.00001,\left.\right|^{2}= \\
91 \%\end{array}$ \\
\hline Plaque & $>6$ months & 0.1 & $1(852)$ & MD $-1.55(-1.79$ to -1.31$)$ & $\begin{array}{l}<0.00001 \text { favours } \\
\mathrm{CHX}\end{array}$ & $N / A$ \\
\hline Plaque & Long term & 0.12 & $4(132)$ & SMD $-1.10(-1.18$ to -0.40$)$ & $\begin{array}{l}<0.002 \text { favours } \\
\mathrm{CHX}\end{array}$ & $P=0.02, \mid 2=71 \%$ \\
\hline
\end{tabular}

$\mathrm{CHX}=$ chlorhexidine $\mathrm{Cl}=$ confidence interval; conc = concentration; $\mathrm{MD}=$ mean difference; $\mathrm{N} / \mathrm{A}=$ not applicable; $\mathrm{SMD}=$ standardised mean difference.

There were no subgroup differences between the different chlorhexidine concentrations, therefore the overall effect for all concentrations combined is reported. 


\begin{tabular}{|c|c|c|c|c|c|c|c|}
\hline Time & Study ID & Comparison & Index & $\begin{array}{l}\text { CHX mean } \\
\text { (SD) }\end{array}$ & $\begin{array}{l}\text { Control mean } \\
\text { (SD) }\end{array}$ & Total n & Notes \\
\hline 4 to 6 weeks & $\begin{array}{l}\text { Anauate-Net- } \\
\text { to } 2014\end{array}$ & $\begin{array}{l}\mathrm{CHX} 0.12 \% \text { vs } \\
\text { placebo }\end{array}$ & PBS & $0.9(0.6)$ & $0.7(0.4)$ & 40 & $\begin{array}{l}\text { Did not report GI. Quote: "..no statistically signifi- } \\
\text { cant differences were detected among groups" }\end{array}$ \\
\hline \multirow[t]{6}{*}{7 to 12 weeks } & $\begin{array}{l}\text { Anderson } \\
1997\end{array}$ & $\begin{array}{l}\mathrm{CHX} 0.12 \% \text { vs } \\
\text { placebo }\end{array}$ & $\mathrm{Gl}$ & 0.345 & 0.895 & 28 & $\begin{array}{l}\text { Reported mean GI by surface + SD. No overall SD. } \\
\text { We calculated overall mean. Quote: "The means } \\
\text { of the ...gingival indices did not show any sig- } \\
\text { nificant differences }(P<0.05) 1 \text { or } 2 \text { months after } \\
\text { baseline. However there were significant differ- } \\
\text { ences }(P<0.05) \text { in the changes recorded at } 30 \text { and } \\
60 \text { days at all sites in.....the experimental group" }\end{array}$ \\
\hline & $\begin{array}{l}\text { de la Rosa } \\
1888 b\end{array}$ & $\begin{array}{l}\mathrm{CHX} 0.12 \% \text { vs } \\
\text { placebo }\end{array}$ & $\begin{array}{l}\text { PMGI severity } \\
\text { (mean score } \\
\text { of all sites } \\
\text { graded) }\end{array}$ & 0.1413 & 0.2902 & 92 & $\begin{array}{l}\text { Did not report GI or a SD. Quote: "..the effect of } \\
\text { the chlorhexidine rinse on the occurrence and } \\
\text { severity of gingivitis amounted to a } 51 \% \text { reduc- } \\
\text { tion of the disease compared to the placebo } \\
\text { rinse... differences were statistically significant" }\end{array}$ \\
\hline & $\begin{array}{l}\text { de la Rosa } \\
\text { 1988a }\end{array}$ & $\begin{array}{l}\mathrm{CHX} 0.12 \% \text { vs } \\
\text { placebo }\end{array}$ & $\begin{array}{l}\text { PMGI severity } \\
\text { (mean score } \\
\text { of all sites } \\
\text { graded) }\end{array}$ & 0.2892 & 0.4526 & 99 & $\begin{array}{l}\text { Did not report GI or a SD. Quote: "..the gingivi- } \\
\text { tis reductions were } 34 \% \text { and } 36 \% \text { for occurrence } \\
\text { and severity respectively...differences were sta- } \\
\text { tistically significant" }\end{array}$ \\
\hline & Eaton 1997 & $\begin{array}{l}\mathrm{CHX} 0.12 \% \text { vs } \\
\text { placebo }\end{array}$ & $\mathrm{mGl}$ & $0.42(0.383)$ & $0.55(0.382)$ & 98 & $\begin{array}{l}\text { Did not report GI. Quote: "..the pooled mean } \mathrm{mGI} \\
\text { score improved by } 25 \% \text { from } 0.56 \text { at baseline } \\
\text { to } 0.42 \text { at } 12 \text { weeks in the } \mathrm{ChD}(\mathrm{CHX}) \text { group but } \\
\text { showed no change ( } 0.54 \text { to } 0.55 \text { ) in the placebo } \\
\text { group" }\end{array}$ \\
\hline & Ferretti 1987 & $\begin{array}{l}\mathrm{CHX} 0.12 \% \text { vs } \\
\text { placebo }\end{array}$ & PMGI & 0.8 & 1.94 & 33 & $\begin{array}{l}\text { Did not report } G \text { I and SD depicted in graph but } \\
\text { not reported. Quote: "Significant reductions in ... } \\
\text { gingivitis scores were seen on days } 33(P<0.0001) \\
\text { and } 60 \ldots(P<0.001) \text { for those patients using } \\
\text { chlorhexidine rinse" }\end{array}$ \\
\hline & Segreto 1986 & $\begin{array}{l}0.2 \% \text { vs } 0.12 \% \\
\text { vs placebo }\end{array}$ & $\mathrm{GI}$ & $0.4112 / 0.3640$ & 0.5039 & 454 & $\begin{array}{l}\text { Did not report a SD. Quote: "Gingivitis severity } \\
\text { by the GI method was... significantly lower at } 3 \\
\text { months for both chlorhexidine groups compared } \\
\text { to the placebo group. Differences ranged from } \\
28-46 \% \text { and averaged } 37 \% \text { for the } 0.12 \% \text { group. } \\
\text { Differences ranged from } 18-40 \% \text { for the } 0.20 \% \\
\text { group and averaged } 29 \% "\end{array}$ \\
\hline
\end{tabular}


Table 6. Studies with a gingival inflammation outcome not included in meta-analyses (Continued)

1.69

1.86 placebo

\begin{tabular}{|c|c|c|c|c|c|c|}
\hline & & placebo & & & & \\
\hline \multirow[t]{3}{*}{6 months } & Fine 1985 & $\begin{array}{l}\mathrm{CHX}+\mathrm{OH} \text { vs } \\
\mathrm{OH}\end{array}$ & $\begin{array}{l}\text { Not clear. } \\
\text { Quote: "a gin- } \\
\text { gival inflam- } \\
\text { mation Index" }\end{array}$ & NR & $N R$ & 83 \\
\hline & $\begin{array}{l}\text { Hoffmann } \\
2001\end{array}$ & $\begin{array}{l}\mathrm{CHX} 0.1 \%, \\
\mathrm{CHX} 0.06 \% \text {, } \\
\mathrm{CHX} 0.06 \%+\mathrm{F} \\
\text { vs control }\end{array}$ & $\mathrm{GI}$ & $\begin{array}{l}\text { Median } \\
0.15 / 0.29 / 0.34\end{array}$ & Median 0.45 & 58 \\
\hline & $\begin{array}{l}\text { Overholser } \\
1990\end{array}$ & $\begin{array}{l}\mathrm{CHX} 0.12 \% \text { vs } \\
\text { control }\end{array}$ & $\mathrm{mGl}$ & $\begin{array}{l}0.81 \text { (SE } \\
0.065)\end{array}$ & $\begin{array}{l}1.166 \text { (SE } \\
0.063)\end{array}$ & 83 \\
\hline
\end{tabular}

$\mathrm{CHX}=$ chlorhexidine; $\mathrm{F}$ = fluoride; $\mathrm{NR}=$ not reported $\mathrm{OH}$ = oral hygiene; $\mathrm{SD}=$ standard deviation; $\mathrm{SE}=$ standard error. Total $\mathrm{n}$ is the number of participants analysed in the study arms relevant to the review.

PBS (Papillary Bleeding Score, Loesche 1979) is measured on a 0-5 increasing scale.

GI (Gingival Index, Löe 1967; Löe and Silness 1963) is measured on a 0-3 increasing scale.

PMGI (Papillary Marginal Gingivitis Index, de la Rosa and Sturzenberger 1976) is measured on a 0-3 increasing scale.

mGI (modified Gingival Index, Lobene 1986) is measured on a 0-4 increasing scale.

Table 7. Studies with a gingival bleeding outcome not included in meta-analyses

\begin{tabular}{|c|c|c|c|c|c|c|c|}
\hline Time & Study ID & Comparison & Index & $\begin{array}{l}\text { CHX mean } \\
\text { (SD) }\end{array}$ & $\begin{array}{l}\text { Control mean } \\
\text { (SD) }\end{array}$ & Total $\mathbf{n}$ & Notes \\
\hline \multirow[t]{2}{*}{4 to 6 weeks } & Axelsson 1987 & $\begin{array}{l}\mathrm{CHX} 0.2 \% \text { vs } \\
\mathrm{CHX} 0.1 \% \text { vs } \\
\text { placebo }\end{array}$ & $\begin{array}{l}\text { Mean } \% \text { of } \\
\text { gingival units } \\
\text { with GI score } \\
2 \text { or } 3\end{array}$ & $12 \% / 11 \%$ & $25 \%$ & 64 & $\begin{array}{l}\text { Did not report a SD. Quote: "In all...study groups, } \\
\text { the } \% \text { of gingival units scored GI } 2+3 \text { was reduced } \\
\text { between baseline..and end of trial" }\end{array}$ \\
\hline & Graziani 2015 & $\begin{array}{l}\mathrm{CHX} 0.2 \%+\mathrm{Alc} \\
\text { vs } \mathrm{CHX} 0.2 \% \\
\text { no Alc vs } \mathrm{CHX}\end{array}$ & FMBS /BOP & Not clear & $13.47 \%$ & 70 & $\begin{array}{l}\text { Partial reporting of outcome data. Quote: "Statis- } \\
\text { tically significant decreases in mean full-mouth } \\
\text { scores of gingival inflammation were noted for }\end{array}$ \\
\hline
\end{tabular}
groups had significantly lower... gingivitis scores than the respective control groups. Overall, the active group had a $10.27 \%$ reduction in the gingival index...compared to insignificant changes in the control groups"

Outcomes reported in graphs which are difficult to decipher. Quote: "Whilst there was a general improvement in all... groups of patients, no one group was statistically significantly different from the other..."

No SD. At 3 months "..only the $0.1 \% \mathrm{CHX}$ was different from the control". At 6 months "...the $0.1 \%$ $\mathrm{CHX}$ showed significant differences...in the GI.. when compared to the $0.06 \% \mathrm{CHX} / \mathrm{F}^{\prime \prime}(\mathrm{P}=0.043)$

Did not report a SD. Quote: "PX (CHX) inhibited gingivitis development by $26.8 \%(\mathrm{P}<0.001)$ at 3 months and by $30.5 \%(P<0.001)$ at 6 months, compared to the control 
Table 7. Studies with a gingival bleeding outcome not included in meta-analyses (Continued) +ADS vs con-

trol rinse

all experimental study groups at day $35 \mathrm{com}$ pared to baseline. Between-group changes in FMBS...were statistically significant only when $\mathrm{CHX} 2$ (mean difference of $43.4 \pm 22.4, \mathrm{P}=0.05$ ) and $\mathrm{CHX} 3$ (mean difference of $46.1 \pm 23.1, \mathrm{P}=$ $0.05)$ were compared to the CTRL group" (note: $\mathrm{CHX} 2=\mathrm{CHX}$ no Alc, $\mathrm{CHX} 3=\mathrm{CHX}+\mathrm{ADS})$

\begin{tabular}{|c|c|c|c|c|c|c|}
\hline Sanz 1989 & $\begin{array}{l}\mathrm{CHX} 0.12 \% \text { vs } \\
\text { placebo }\end{array}$ & $\begin{array}{l}\text { Mean } \% \text { sites } \\
\text { GI score } 2 \text { and } \\
3\end{array}$ & $18.78 \%$ & $31.31 \%$ & 38 & $\begin{array}{l}\text { Did not report a SD. Quote: "Beginning at } 4 \text { weeks } \\
\text { of rinsing, gingival bleeding was significantly } \\
\text { lower in the CHX group compared to the place- } \\
\text { bo group by an average of } 41.6 \%(P<0.05) \text {. At } 6 \\
\text { weeks that reduction was } 40 \%,(P<0.05) "\end{array}$ \\
\hline
\end{tabular}

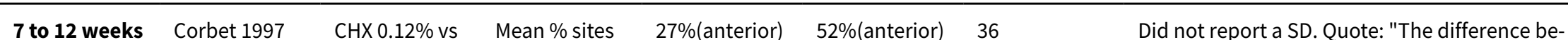

placebo Gl score 2 and

3

$42 \%($ posteri-

$75 \%$ (posteri-

or)

\begin{tabular}{lllll}
\hline Segreto 1986 & CHX 0.2\% vs & Mean \% sites & Examiner A: & Examiner A: \\
& CHX 0.12\% vs & GI score 2 and & $3.4 \% / 2 \%$ & $3.81 \%$ \\
placebo & 3 & & Examiner B: & Examiner B: \\
& & & $7.2 \% / 6.2 \%$ & $14.9 \%$
\end{tabular}

454

Did not report a SD. Quote: "The difference between the mean percentage of GB of the test and control groups at 3 months was highly significant $(\mathrm{P}<0.001)^{\prime \prime}$

Did not report a SD.

0.2\% Quote: "Bleeding was 31\% lower (range $11-52 \%)$ compared to the placebo group" ( $P>$ 0.05

$\mathbf{0 . 1 2} \%$ Quote: "..gingival bleeding was significantly lower by an average of $53 \%$ for both examiners (range 48-59\%)" ( $\mathrm{P} \leq 0.05)$

\begin{tabular}{|c|c|c|c|c|c|c|c|}
\hline \multirow[t]{2}{*}{6 months } & Banting 1989 & $\begin{array}{l}\mathrm{CHX} 0.12 \% \text { vs } \\
\text { placebo }\end{array}$ & $\begin{array}{l}\text { Mean } \% \text { sites } \\
\text { Gl score } 2 \text { and } \\
3\end{array}$ & $2.41 \%$ & $4.12 \%$ & 383 & $\begin{array}{l}\text { Did not report a SD. Regarding outcomes at } 6 \\
\text { months to } 2 \text { years: Quote: "Subjects in the treat- } \\
\text { ment group...displayed between } 42 \% \text { and } 51 \% \\
\text { fewer sites with moderate to severe gingivitis (GI } \\
\text { scores of } 2 \text { or } 3 \text { ) compared with subjects in the } \\
\text { control group" }(\mathrm{P}<0.0001)\end{array}$ \\
\hline & Charles 2004 & $\begin{array}{l}\mathrm{CHX} 0.12 \% \text { vs } \\
\text { control }\end{array}$ & $\begin{array}{l}\text { Mean } \% \text { sites } \\
\text { Gl score } 2 \text { and } \\
3\end{array}$ & $11.01 \%$ & $20.65 \%$ & 1156 sites & $\begin{array}{l}\% \text { of bleeding sites in each group is presented. } \\
\text { There is a unit of analysis error (number of sites } \\
\text { rather than number of subjects). Quote: "There } \\
\text { was a considerable reduction in percent bleeding } \\
\text { sites in the chlorhexidine...groups at } 6 \text { months } \\
\text { compared with both control and baseline" }\end{array}$ \\
\hline
\end{tabular}


Table 7. Studies with a gingival bleeding outcome not included in meta-analyses (Continued)

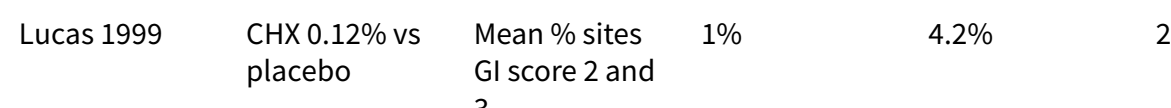

3

CHX $0.12 \%$ vs \% sites GI Graph

placebo score 2 and 3

Graph

Graph

130

130

Did not report a SD. Quote: "Although the percentage of bleeding surfaces in the chlorhexidine group was less than in the placebo group on days $90(33 \%)$ and $180(76 \%)$, the differences were not significant" $(P=0.07)$

$\%$ bleeding sites reported incompletely in text and also in a graph. Did not report a SD.

Quote: "At 6 months...the positive control group had significantly fewer bleeding sites than the control group $(. .23 \%$...)"

\begin{tabular}{|c|c|c|c|c|c|c|c|}
\hline $\begin{array}{l}\text { Gingival } \\
\text { bleeding > } 6 \\
\text { months }\end{array}$ & Banting 1989 & $\begin{array}{l}\mathrm{CHX} 0.12 \% \text { vs } \\
\text { placebo }\end{array}$ & $\begin{array}{l}\text { Mean } \% \text { sites } \\
\text { GI score } 2 \text { and } \\
3\end{array}$ & $4.41 \%$ & $8.88 \%$ & 272 & $\begin{array}{l}\text { Did not report a SD. Regarding outcomes at } 6 \\
\text { months to } 2 \text { years: Quote: "Subjects in the treat- } \\
\text { ment group...displayed between } 42 \% \text { and } 51 \% \\
\text { fewer sites with moderate to severe gingivitis (GI } \\
\text { scores of } 2 \text { or } 3 \text { ) compared with subjects in the } \\
\text { control group" ( } P<0.0001 \text { ) }\end{array}$ \\
\hline
\end{tabular}

$\mathrm{ADS}=$ antidiscolouration system; $\mathrm{Alc}=$ alcohol; $\mathrm{BOP}=$ bleeding on probing; $\mathrm{CHX}=$ chlorhexidine; FMBS = Full-Mouth Bleeding Score; $\mathrm{GI}=\mathrm{Gingival}$ Index; $\mathrm{SD}=\mathrm{standard}$ deviation Total $\mathrm{n}$ is the number of participants analysed in the study arms relevant to the review.

Table 8. Studies with a plaque outcome not included in meta-analyses

\begin{tabular}{|c|c|c|c|c|c|c|c|}
\hline Time & Study ID & Comparison & Index & $\begin{array}{l}\text { CHX mean } \\
\text { (SD) }\end{array}$ & $\begin{array}{l}\text { Control mean } \\
\text { (SD) }\end{array}$ & Total $\mathbf{n}$ & Notes \\
\hline 4 to 6 weeks & Sanz 1989 & $\begin{array}{l}\mathrm{CHX} 0.12 \% \text { vs } \\
\text { placebo }\end{array}$ & $\mathrm{PI}$ & 0.452 & 0.9907 & 38 & Did not report a SD \\
\hline \multirow[t]{3}{*}{7 to 12 weeks } & $\begin{array}{l}\text { Anderson } \\
1997\end{array}$ & $\begin{array}{l}\mathrm{CHX} 0.12 \% \text { vs } \\
\text { placebo }\end{array}$ & $\mathrm{PI}$ & $0.3175^{\star}$ & $0.8425^{\star}$ & 28 & $\begin{array}{l}\text { Mean } \mathrm{Gl} \text { by surface }+\mathrm{SD} \text { reported. We calculat- } \\
\text { ed the overall mean. No overall SD }\end{array}$ \\
\hline & $\begin{array}{l}\text { de la Rosa } \\
\text { 1988a }\end{array}$ & $\begin{array}{l}\mathrm{CHX} 0.12 \% \text { vs } \\
\text { placebo }\end{array}$ & $\mathrm{TQH}$ & NR & NR & 99 & $\begin{array}{l}\text { Quote: "The reductions in dental plaque were } \\
\text { not statistically significant.." }\end{array}$ \\
\hline & Segreto 1986 & $\begin{array}{l}0.2 \% \text { vs } 0.12 \% \\
\text { vs placebo }\end{array}$ & $\mathrm{TQH}$ & $1.14 / 1.01$ & 1.58 & 451 & Did not report a SD \\
\hline
\end{tabular}


Table 8. Studies with a plaque outcome not included in meta-analyses (Continued)

\begin{tabular}{|c|c|c|c|c|c|c|c|}
\hline & Weitz 1992 & $\begin{array}{l}\mathrm{CHX} 0.12 \% \text { vs } \\
\text { placebo }\end{array}$ & $\mathrm{PI}$ & 1.84 & 2.21 & 36 & Did not report a SD \\
\hline \multirow[t]{4}{*}{6 months } & Banting 1989 & $\begin{array}{l}\mathrm{CHX} 0.12 \% \text { vs } \\
\text { placebo }\end{array}$ & $\mathrm{TQH}$ & Graph & Graph & 383 & $\begin{array}{l}\text { Data presented in a graph. Did not report a SD. } \\
\text { Quote: "Subjects in the treatment group had } \\
\text { significantly lower mean plaque scores than } \\
\text { those in the control group at six months, and at } \\
\text { one and two years. The difference between the } \\
\text { groups ranged from } 35 \% \text { to } 46 \% \text { " }\end{array}$ \\
\hline & $\begin{array}{l}\text { Jayaprakash } \\
2007\end{array}$ & $\begin{array}{l}\mathrm{CHX} 0.05 \% / \\
\mathrm{CHX} 0.05 \%+\mathrm{F} \text { vs } \\
\text { placebo }\end{array}$ & $\mathrm{PI}$ & $0.0813 / 0.0459$ & 0.1189 & 100 & Did not report a SD \\
\hline & Lucas 1999 & $\begin{array}{l}\mathrm{CHX} 0.12 \% \text { vs } \\
\text { placebo }\end{array}$ & $\mathrm{OHI}-\mathrm{S}$ & 0.33 & 0.59 & 20 & Did not report a SD \\
\hline & Sanz 1994 & $\begin{array}{l}\mathrm{CHX} 0.12 \% \text { vs } \\
\text { placebo }\end{array}$ & $\mathrm{PI}$ & Graph & Graph & 130 & $\begin{array}{l}\text { Data presented in a graph. Did not report a SD. } \\
\text { Quote: "...reductions in Plaque Index... were } \\
\text { statistically significant for the positive control } \\
\text { group...compared with the reduction obtained } \\
\text { with the control group.. These reductions were } \\
41 \% \text { (positive control)... after } 3 \text { months and } \\
35 \% \text {..after } 6 \text { months.." }\end{array}$ \\
\hline $\begin{array}{l}\text { Plaque > } 6 \\
\text { months }\end{array}$ & Banting 1989 & $\begin{array}{l}\mathrm{CHX} 0.12 \% \text { vs } \\
\text { placebo }\end{array}$ & $\mathrm{TQH}$ & Graph & Graph & 272 & $\begin{array}{l}\text { Data presented in a graph. Did not report a SD. } \\
\text { Quote: "Subjects in the treatment group had } \\
\text { significantly lower mean plaque scores than } \\
\text { those in the control group at six months, and at } \\
\text { one and two years. The difference between the } \\
\text { groups ranged from } 35 \% \text { to } 46 \% "\end{array}$ \\
\hline
\end{tabular}

$\mathrm{CHX}=$ chlorhexidine $; \mathrm{F}=$ Fluoride $\mathrm{NR}=$ not reported $; \mathrm{SD}=$ standard deviation.

Total $\mathrm{n}$ is the number of participants analysed in the study arms relevant to the review.

PI (Plaque Index, Silness and Löe 1964) is measured on a 0-3 increasing scale.

TQH (Turesky modification of the Quigley and Hein Index, Turesky 1970) is measured on a 0-5 increasing scale.

OHI-S (Simplified Oral Hygiene Index, Greene and Vermillion 1964) is measured on a 0-3 increasing scale. 
Table 9. Studies with a calculus outcome not included in meta-analyses

\begin{tabular}{|c|c|c|c|c|c|c|c|}
\hline Time & Study ID & Comparison & Index & $\begin{array}{l}\text { CHX mean } \\
\text { (SD) }\end{array}$ & $\begin{array}{l}\text { Control mean } \\
\text { (SD) }\end{array}$ & Total $\mathbf{n}$ & Notes \\
\hline 4 to 6 weeks & $\begin{array}{l}\text { Anderson } \\
1997\end{array}$ & $\begin{array}{l}\mathrm{CHX} 0.12 \% \text { vs } \\
\text { placebo }\end{array}$ & RI & 0.1075 & 0.0475 & 29 & $\begin{array}{l}\text { Mean RI by surface }+ \text { SD reported. We calculated } \\
\text { overall mean. No overall SD }\end{array}$ \\
\hline \multirow[t]{3}{*}{7 to 12 weeks } & $\begin{array}{l}\text { Anderson } \\
1997\end{array}$ & $\begin{array}{l}\mathrm{CHX} 0.12 \% \text { vs } \\
\text { placebo }\end{array}$ & RI & 0.0875 & 0.0525 & 28 & $\begin{array}{l}\text { Mean RI by surface + SD reported. We calculated } \\
\text { overall mean. No overall SD }\end{array}$ \\
\hline & Charles 2004 & $\begin{array}{l}\mathrm{CHX} 0.12 \% \text { vs } \\
\text { control }\end{array}$ & VM & 0.37 (SD NR) & 0.11 (SD NR) & 74 & Did not report a SD \\
\hline & Sanz 1994 & $\begin{array}{l}\mathrm{CHX} 0.12 \% \text { vs } \\
\text { placebo }\end{array}$ & VM & Graph & Graph & 130 & $\begin{array}{l}\text { Data presented in graph. Did not report a SD. } \\
\text { Quote: "All groups developed calculus after the } \\
\text { initial cleaning at baseline. This increase was on- } \\
\text { ly statistically significant for the positive con- } \\
\text { trol group compared with the control group at } 6 \\
\text { months" }\end{array}$ \\
\hline \multirow{3}{*}{$\begin{array}{l}\text { Calculus } 6 \\
\text { months }\end{array}$} & Charles 2004 & $\begin{array}{l}\mathrm{CHX} 0.12 \% \text { vs } \\
\text { control }\end{array}$ & VM & 0.45 (SD NR) & 0.21 (SD NR) & 73 & Did not report a SD \\
\hline & $\begin{array}{l}\text { Grossman } \\
1986\end{array}$ & $\begin{array}{l}\mathrm{CHX} 0.12 \% \text { vs } \\
\text { placebo }\end{array}$ & Not specified & NR & NR & 380 & $\begin{array}{l}\text { Quote: "Supragingival calculus was higher in } \\
\text { the group using chlorhexidine but this increase } \\
\text { in calculus did not diminish the therapeutic ef- } \\
\text { fects of chlorhexidine since subjects with signifi- } \\
\text { cant increases in calculus also had significant de- } \\
\text { creases in gingivitis" }\end{array}$ \\
\hline & Sanz 1994 & $\begin{array}{l}\mathrm{CHX} 0.12 \% \text { vs } \\
\text { placebo }\end{array}$ & VM & Graph & Graph & 130 & $\begin{array}{l}\text { Data presented in a graph. Did not report a SD. } \\
\text { Quote: "All groups developed calculus after the } \\
\text { initial cleaning at baseline. This increase was on- } \\
\text { ly statistically significant for the positive con- } \\
\text { trol group compared with the control group at } 6 \\
\text { months" }\end{array}$ \\
\hline
\end{tabular}


$\mathrm{CHX}=$ chlorhexidine; $\mathrm{NR}=$ not reported $\mathrm{SD}=$ standard deviation

Total $\mathrm{n}$ is the number of participants analysed in the study arms relevant to the review.

RI (Retention Index, Björby and Löe 1966) is measured on a 0-3 increasing scale.

VM (Volpe-Manhold Calculus Index, Manhold 1965; Volpe 1965) measures calculus present on the lingual surface of the lower 6 anterior teeth. Calculus is measured in 3 planes using a standard periodontal probe.The greatest value allowed for any 1 plane is 3 units, therefore the maximum score per tooth is 9 units or 54 units per subject. The mean per subject score is obtained by dividing the total calculus score by the number of lower anterior teeth. A mean calculus score for the group is then calculated.

\section{Table 10. Studies with a staining outcome not included in meta-analyses}

\begin{tabular}{lllll}
\hline Time Study ID Comparison Index & $\begin{array}{l}\text { CHX mean } \\
\text { (SD/SE) or n } \\
(\%)\end{array}$ & $\begin{array}{l}\text { Control mean Total } \\
\text { (SD) }\end{array}$ & Notes
\end{tabular}

\begin{tabular}{|c|c|c|c|c|c|c|c|}
\hline \multirow[t]{4}{*}{4 to 6 weeks } & Axelsson 1987 & $\begin{array}{l}\mathrm{CHX} \\
0.2 \% / 0.1 \% \text { vs } \\
\text { control }\end{array}$ & DI (Lobene) & $0.1171(0.297)$ & 0 & 64 & $\begin{array}{l}\text { Not included in meta-analysis as con- } \\
\text { trol mean is } 0 . \text { We combined data from } \\
\text { the } 0.2 \% \text { and } 0.1 \% \mathrm{CHX} \text { groups }\end{array}$ \\
\hline & Bhat 2014 & $\begin{array}{l}\mathrm{CHX} 0.2 \% \text { vs } \\
\text { control }\end{array}$ & $\begin{array}{l}\text { Reported number and } \\
\text { proportion of partici- } \\
\text { pants with mild or mod- } \\
\text { erate 'discolouration' in } \\
\text { text }\end{array}$ & $20(91 \%)$ & 0 & 44 & $\begin{array}{l}\text { Not clear whether discolouration re- } \\
\text { lates to teeth or oral tissues or both }\end{array}$ \\
\hline & $\begin{array}{l}\text { Flotra } 1972 \\
\& 1971 \text { (4- } \\
\text { month study } \\
\text { that reports } \\
\text { this outcome } \\
\text { at } 4 \text { weeks) }\end{array}$ & $\begin{array}{l}\text { CHX } \\
0.2 \% / 0.1 \% / 0.1 \% \\
\text { (acetate) vs } \\
\text { control }\end{array}$ & $\begin{array}{l}\text { No index } \\
\text { Reported narratively }\end{array}$ & $\begin{array}{l}12 \% \text { of tooth } \\
\text { surfaces with- } \\
\text { out fillings } \\
62 \% \text { of silicate } \\
\text { fillings }\end{array}$ & NR & $\begin{array}{l}48 \text { (at } 4 \\
\text { weeks) }\end{array}$ & $\begin{array}{l}\text { Quote: "..12\% of the tooth surfaces } \\
\text { without fillings became discolored } \\
\text { within the first } 4 \text { weeks of the experi- } \\
\text { ment...this happened more frequently } \\
\text { on the interproximal surfaces than on } \\
\text { the labial surfaces (ratio2:1). Sixty-two } \\
\text { per cent of the silicate fillings in these } \\
\text { areas were discolored.." }\end{array}$ \\
\hline & Hase 1995 & $\begin{array}{l}\text { CHX } 0.2 \% \text { vs } \\
\text { placebo }\end{array}$ & $\begin{array}{l}\text { Subjective of partici- } \\
\text { pants regarding staining } \\
\text { of teeth and/or tongue } \\
\text { using VAS }\end{array}$ & 38 (SE 7) & 9 (SE 1) & 39 & $\begin{array}{l}\text { Teeth and/or tongue staining reported } \\
\text { together. Data estimated from a graph }\end{array}$ \\
\hline
\end{tabular}




\begin{tabular}{|c|c|c|c|c|c|c|c|}
\hline & $\begin{array}{l}\text { López-Jornet } \\
2012\end{array}$ & $\begin{array}{l}\mathrm{CHX} 0.2 \% \text { vs } \\
\text { placebo }\end{array}$ & $\begin{array}{l}\text { Registration of side ef- } \\
\text { fects including den- } \\
\text { ture/dental staining ( } \mathrm{n} \\
\text { and \%) }\end{array}$ & $2(5.71 \%)$ & $3(8.57 \%)$ & 70 & $\begin{array}{l}\text { Denture and dental staining reported } \\
\text { together }\end{array}$ \\
\hline & Turkoglu 2009 & $\begin{array}{l}\mathrm{CHX} \text { (conc not } \\
\text { reported) vs } \\
\text { placebo }\end{array}$ & $\begin{array}{l}\text { No index } \\
\text { Reported narratively }\end{array}$ & $14(56 \%)$ & Assumed 0 & 50 & $\begin{array}{l}\text { Quote: "Of the } 25 \text { subjects who rinsed } \\
\text { their mouth with } \mathrm{CHX} \text { mouthrinse..14 } \\
\text { showed discolouration of teeth and/or } \\
\text { tongue" }\end{array}$ \\
\hline & Zimmer 2006 & $\begin{array}{l}\mathrm{CHX} 0.06 \%+\mathrm{F} \\
+\mathrm{OH} \text { vs } \mathrm{OH}\end{array}$ & $\begin{array}{l}\text { Staining of teeth and } \\
\text { tongue registered at fi- } \\
\text { nal examination }(\mathrm{n})\end{array}$ & 4 & 0 & 78 & $\begin{array}{l}\text { Stain on teeth/tongue reported to- } \\
\text { gether. If more than } 1 \text { side effect was } \\
\text { present, only the most relevant was } \\
\text { listed i.e. side effects were reported } \\
\text { with no double counting }\end{array}$ \\
\hline \multirow[t]{4}{*}{7 to 12 weeks } & Charles 2004 & $\begin{array}{l}\mathrm{CHX} 0.12 \% \text { vs } \\
\text { control }\end{array}$ & DI (Lobene) & 1.61 (SD NR) & 0.01 (SD NR) & 74 & Did not report a SD \\
\hline & $\begin{array}{l}\text { Grossman } \\
1989\end{array}$ & $\begin{array}{l}\mathrm{CHX} 0.12 \% \text { vs } \\
\text { placebo }\end{array}$ & $\begin{array}{l}\text { Not specified Quote: } \\
\text { "Photographs of facial } \\
\text { surfaces of the } 12 \text { ante- } \\
\text { rior teeth (maxillary and } \\
\text { mandibular, cuspid to } \\
\text { cuspid) were graded for } \\
\text { stain intensity and cov- } \\
\text { erage" }\end{array}$ & 4.66 (SD NR) & 2.59 (SD NR) & 246 & Did not report a SD \\
\hline & Sanz 1994 & $\begin{array}{l}\mathrm{CHX} 0.12 \% \text { vs } \\
\text { placebo }\end{array}$ & Not specified & NR & NR & 130 & $\begin{array}{l}\text { Data presented in a graph at } 6 \text { months } \\
\text { only See } 6 \text {-month results below }\end{array}$ \\
\hline & Zimmer 2006 & $\begin{array}{l}\mathrm{CHX} 0.06 \%+\mathrm{F} \\
+\mathrm{OH} \text { vs } \mathrm{OH}\end{array}$ & $\begin{array}{l}\text { Staining of teeth and } \\
\text { tongue registered at fi- } \\
\text { nal examination }(\mathrm{n})\end{array}$ & 6 & 0 & 78 & $\begin{array}{l}\text { Stain on teeth/tongue reported to- } \\
\text { gether. If more than } 1 \text { side effect was } \\
\text { present, only the most relevant was } \\
\text { listed i.e. side effects were reported } \\
\text { with no double counting }\end{array}$ \\
\hline \multirow[t]{2}{*}{6 months } & Charles 2004 & $\begin{array}{l}\mathrm{CHX} 0.12 \% \text { vs } \\
\text { control }\end{array}$ & DI (Lobene) & 2.08 (SD NR) & 0.01 (SD NR) & 73 & Did not report a SD \\
\hline & $\begin{array}{l}\text { Grossman } \\
1986\end{array}$ & $\begin{array}{l}\mathrm{CHX} 0.12 \% \text { vs } \\
\text { placebo }\end{array}$ & Not specified & NR & NR & 380 & $\begin{array}{l}\text { Outcome data not reported. Quote: } \\
\text { "Some extrinsic tooth staining was ob- } \\
\text { served in the chlorhexidine group" }\end{array}$ \\
\hline
\end{tabular}


Table 10. Studies with a staining outcome not included in meta-analyses (Continued)

\begin{tabular}{|c|c|c|c|c|c|c|}
\hline $\begin{array}{l}\text { Grossman } \\
1989\end{array}$ & $\begin{array}{l}\mathrm{CHX} 0.12 \% \text { vs } \\
\text { placebo }\end{array}$ & $\begin{array}{l}\text { Not specified Quote: } \\
\text { "Photographs of facial } \\
\text { surfaces of the } 12 \text { ante- } \\
\text { rior teeth (maxillary and } \\
\text { mandibular, cuspid to } \\
\text { cuspid) were graded for } \\
\text { stain intensity and cov- } \\
\text { erage" }\end{array}$ & 5.15 (SD NR) & 2.75 (SD NR) & 246 & Did not report a SD \\
\hline $\begin{array}{l}\text { Hoffmann } \\
2001\end{array}$ & $\begin{array}{l}\mathrm{CHX} 0.1 \% / \\
0.06 \% / 0.06 \% \\
+\mathrm{F} \text { vs control }\end{array}$ & DI (Lang and Räber) & $\begin{array}{l}1.13 / 1.02 / 1.06 \\
\text { (SD NR) }\end{array}$ & 0.38 (SD NR) & 58 & Median only. Did not report a SD \\
\hline Sanz 1994 & $\begin{array}{l}\mathrm{CHX} 0.12 \% \text { vs } \\
\text { placebo }\end{array}$ & $\begin{array}{l}\text { Not specified Quote: } \\
\text { "Photographs of facial } \\
\text { surfaces of the } 12 \text { ante- } \\
\text { rior teeth (maxillary and } \\
\text { mandibular, cuspid to } \\
\text { cuspid) were graded for } \\
\text { stain intensity and cov- } \\
\text { erage" }\end{array}$ & Graph & Graph & 130 & $\begin{array}{l}\text { Data presented in graph Did not report } \\
\text { a SD } \\
\text { Quote: "Statistically significant more } \\
\text { overall staining, more intense staining } \\
\text { and stain coverage per tooth were de- } \\
\text { tected for the positive control group... } \\
\text { compared with the control group" }\end{array}$ \\
\hline
\end{tabular}

$\mathrm{CHX}=$ chlorhexidine conc = concentration; $\mathrm{F}=$ fluoride; $\mathrm{NR}=$ not reported $; \mathrm{OH}=$ oral hygiene; $\mathrm{SD}=$ standard deviation; $\mathrm{SE}=$ standard error; $\mathrm{VAS}=$ visual analogue $\mathrm{Scale}$. Total $\mathrm{n}$ is the number of participants analysed in the study arms relevant to the review.

DI (Discolouration Index, Lobene 1968): gingival and body regions of the tooth are scored for intensity (0-3 increasing scale) and severity (0-3 increasing scale).

DI (Discolouration Index, Lang and Räber 1981) is measured on a 0-3 increasing scale.

SI (Staining Index): the buccal surfaces of the 8 central incisors were divided into 3 areas: incisal, approximal and gingival according to Lobene 1968 and Grundemann 2000 and a

SI was used to record the dichotomous presence or absence of staining in each area and to calculate the percentage of the total area showing staining. 


\section{AP PE N D I C E S}

\section{Appendix 1. Cochrane Oral Health's Trials Register search strategy}

From July 2014, searches of Cochrane Oral Health's Trials Register were conducted using the Cochrane Register of Studies using the search strategy below:

1 ((plaque or gingivitis or gingiva* or periodont* or "gum disease*"):ti,ab) AND (INREGISTER)

2 ((mouthwash* or mouthrinse* or wash* or rins $\left.{ }^{\star}\right):$ ti,ab) AND (INREGISTER)

3 ((chlorhexidine* or MK-412a or "MK 412a" or MK412a or CHX or Corsodyl or Peridex or Hibident or Prexidine or Parodex or Chlorexil or Peridont or Eludril or Perioxidin or Chlorohex or Savacol or Periogard or Chlorhexamed or Nolvasan or Sebidin or Tubulicid or hibitane):ti,ab) AND (INREGISTER)

4 (\#1 and \#2 and \#3) AND (INREGISTER)

Previous searches of Cochrane Oral Health's Trials Register were undertaken in April 2010 and February 2012 using the Procite software and the search strategy below:

((plaque or gingivitis or gingiva* or periodont* or "gum disease*") AND (mouthwash ${ }^{\star}$ or mouthrinse ${ }^{\star}$ or wash $^{\star}$ or rins $^{\star}$ ) AND (chlorhexidine ${ }^{\star}$ or MK-412a or "MK 412a" or MK412a or CHX or Corsodyl or Peridex or Hibident or Prexidine or Parodex or Chlorexil or Peridont or Eludril or Perioxidin or Chlorohex or Savacol or Periogard or Chlorhexamed or Nolvasan or Sebidin or Tubulicid or hibitane))

\section{Appendix 2. Cochrane Central Register of Controlled Trials (CENTRAL) search strategy}

\#1 MeSH descriptor Dental Plaque, this term only \#2 ((dental or oral or tooth or teeth) and plaque) \#3 MeSH descriptor Gingivitis explode all trees \#4 gingivitis \#5 ((gingiva* near/3 inflamm*) or (gingiva* near/3 bleed ${ }^{\star}$ ) or (gingiva* near/3 pocket ${ }^{\star}$ ) or (gingiva* near/3 attachment $\left.{ }^{\star}\right)$ ) \#6 MeSH descriptor Periodontitis explode all trees \#7 periodont*

\#8 ((haemorrhag* near/5 gum ${ }^{\star}$ ) or (hemorrhag* near/5 gum ${ }^{\star}$ ) or (hemorrag* near/5 gum ${ }^{\star}$ ) or (haemorrhag* near/5 gingiva*) or (hemorrhag* near $/ 5$ gingiva*) or (hemorrag* near $/ 5$ gingiva*))

\#9 ((bleed ${ }^{\star}$ near $/ 5$ gum $\left.^{\star}\right)$ or $\left(\right.$ bleed $^{\star}$ near $/ 5$ gingiva $\left.\left.{ }^{\star}\right)\right)$

\#10 ((inflamm* near/5 gum*) or (inflamm* near/5 gingiva*))

$\# 11$ ((gum next disease) or (disease* near/5 gum $\left.{ }^{\star}\right)$ or (disease near/5 gingiva $\left.{ }^{\star}\right)$ )

\#12 (\#1 OR \#2 OR \#3 OR \#4 OR \#5 OR \#6 OR \#7 OR \#8 OR \#9 OR \#10 OR \#11)

$\# 13$ MeSH descriptor Mouthwashes explode all trees

\#14 (mouthrins ${ }^{\star}$ or "mouth rins" or mouth-rins ${ }^{\star}$ or rinse or rinsing $^{\star}$ or mouthwash* or "mouth wash*" or mouth-wash*)

\#15 ((oral near/3 wash*) or (dental near/3 wash*) or (mouth* near/3 wash*) or (oral near/3 rins*) or (dental near/3 rins $\left.{ }^{\star}\right)$ or $\left(\mathrm{mouth}^{\star}\right.$ near/3 $\left.\left.\operatorname{rins}^{\star}\right)\right)$

\#16 (\#13 OR \#14 OR \#15)

\#17 MeSH descriptor Chlorhexidine, this term only

$\# 18$ chlorhexidine

\#19 (MK-412a or "MK 412a" or MK412a)

$\# 20$ (CHX):ti,ab,kw

\#21 (Corsodyl or Peridex or Hibident or Prexidine or Parodex or Chlorexil or Peridont or Eludril or Perioxidin or Chlorohex or Savacol or Periogard or Chlorhexamed or Nolvasan or Sebidin or Tubulicid or hibitane)

\#22 (\#17 OR \#18 OR \#19 OR \#20 OR \#21)

\#23 (\#12 AND \#16 AND \#22)

\section{Appendix 3. MEDLINE Ovid search strategy}

1. Dental plaque/

2. ((dental or oral or tooth or teeth) and plaque).mp.

3. exp Gingivitis/

4. gingivitis.mp.

5. ((gingiva\$ adj3 inflamm\$) or (gingiva\$ adj3 bleed\$) or (gingiva\$ adj3 pocket\$) or (gingiva\$ adj3 attachment\$)).mp.

6. exp Periodontitis/

7. periodont\$.mp.

8. ((haemorrhag\$ adj5 gum\$) or (hemorrhag\$ adj5 gum\$) or (hemorrag\$ adj5 gum\$) or (haemorrhag\$ adj5 gingiva\$) or (hemorrhag\$ adj5 gingiva\$) or (hemorrag\$ adj5 gingiva\$)).mp. 
9. ((bleed\$ adj5 gum\$) or (bleed\$ adj5 gingiva\$)).mp.

10.((inflamm\$ adj5 gum\$) or (inflamm\$ adj5 gingiva\$)).mp.

11. ((gum adj disease) or (disease $\$$ adj5 gum\$) or (disease adj5 gingiva\$)).mp.

12.or/1-11

13.exp Mouthwashes/

14. (mouthrins\$ or "mouth rins\$" or mouth-rins\$ or rinse\$ or rinsing\$ or mouthwash\$ or "mouth wash\$" or mouth-wash\$).mp.

15.((oral or dental or mouth) adj3 (wash\$ or rins\$)).mp.

16.or/13-15

17.Chlorhexidine/

18.chlorhexidine.mp.

19. (MK-412a or "MK 412a" or MK412a).mp.

20.CHX.ti,ab.

21. (Corsodyl or Peridex or Hibident or Prexidine or Parodex or Chlorexil or Peridont or Eludril or Perioxidin or Chlorohex or Savacol or Periogard or Chlorhexamed or Nolvasan or Sebidin or Tubulicid or hibitane).mp.

22.or/17-21

23.12 and 16 and 22

This subject search was linked to the Cochrane Highly Sensitive Search Strategy (CHSSS) for identifying randomised trials (RCTs) in MEDLINE: sensitivity-maximising version (2008 revision) as referenced in Chapter 6.4.11.1 and detailed in box 6.4.c of the Cochrane Handbook for Systematic Reviews of Interventions, Version 5.1.0 (updated March 2011) (Lefebvre 2011).

1. randomized controlled trial.pt.

2. controlled clinical trial.pt.

3. randomized.ab.

4. placebo.ab.

5. drug therapy.fs.

6. randomly.ab.

7. trial.ab.

8. groups.ab.

9. or/1-8

10. exp animals/ not humans.sh.

11.9 not 10

\section{Appendix 4. Embase Ovid search strategy}

1. Dental plaque/

2. ((dental or oral or tooth or teeth) and plaque).mp.

3. exp Gingivitis/

4. gingivitis.mp.

5. ((gingiva\$ adj3 inflamm\$) or (gingiva\$ adj3 bleed\$) or (gingiva\$ adj3 pocket\$) or (gingiva\$ adj3 attachment\$)).mp.

6. exp Periodontitis/

7. periodont\$.mp.

8. ((haemorrhag\$ adj5 gum\$) or (hemorrhag\$ adj5 gum\$) or (hemorrag\$ adj5 gum\$) or (haemorrhag\$ adj5 gingiva\$) or (hemorrhag\$ adj5 gingiva\$) or (hemorrag\$ adj5 gingiva\$)).mp.

9. ((bleed\$ adj5 gum\$) or (bleed\$ adj5 gingiva\$)).mp.

10. ((inflamm\$ adj5 gum\$) or (inflamm\$ adj5 gingiva\$)).mp.

11. ((gum adj disease) or (disease\$ adj5 gum\$) or (disease adj5 gingiva\$)).mp. [mp=title, original title, abstract, name of substance word, subject heading word, unique identifier]

12.or/1-11

13.exp Mouthwashes/

14. (mouthrins\$ or "mouth rins\$" or mouth-rins\$ or rinse\$ or rinsing\$ or mouthwash\$ or "mouth wash\$" or mouth-wash\$).mp.

15.((oral or dental or mouth) adj3 (wash\$ or rins\$)).mp.

16.or/13-15

17.Chlorhexidine/

18.chlorhexidine.mp.

19.(MK-412a or "MK 412a" or MK412a).mp.

20.CHX.ti,ab.

Chlorhexidine mouthrinse as an adjunctive treatment for gingival health (Review)

Copyright (c) 2017 The Cochrane Collaboration. Published by John Wiley \& Sons, Ltd. 
21. (Corsodyl or Peridex or Hibident or Prexidine or Parodex or Chlorexil or Peridont or Eludril or Perioxidin or Chlorohex or Savacol or Periogard or Chlorhexamed or Nolvasan or Sebidin or Tubulicid or hibitane).mp.

22.or/17-21

23.12 and 16 and 22

The above subject search was linked to adapted version of the Cochrane Embase Project filter for identifying RCTs in Embase Ovid (see http://www.cochranelibrary.com/help/central-creation-details.html for information):

1. Randomized controlled trial/

2. Controlled clinical study/

3. Random\$.ti,ab.

4. randomization/

5. intermethod comparison/

6. placebo.ti,ab.

7. (compare or compared or comparison).ti.

8. ((evaluated or evaluate or evaluating or assessed or assess) and (compare or compared or comparing or comparison)).ab.

9. (open adj label).ti,ab.

10. ((double or single or doubly or singly) adj (blind or blinded or blindly)).ti,ab.

11. double blind procedure/

12. parallel group $\$ 1 . t i, a b$.

13. (crossover or cross over).ti,ab.

14. ((assign\$ or match or matched or allocation) adj5 (alternate or group\$1 or intervention\$1 or patient\$1 or subject\$1 or participant

\$1)).ti,ab.

15. (assigned or allocated).ti,ab.

16. (controlled adj7 (study or design or trial)).ti,ab.

17. (volunteer or volunteers).ti,ab.

18. trial.ti.

19. or/1-18

20. (exp animal/ or animal.hw. or nonhuman/) not (exp human/ or human cell/ or (human or humans).ti.)

21. 19 not 20

\section{Appendix 5. CINAHL EBSCO search strategy}

S1 MH "Dental Plaque+"

S2 ((dental or oral or tooth or teeth) and plaque)

S3 MH "Gingivitis+"

S4 gingivitis

S5 (gingiva* N3 inflamm*) or (gingiva* N3 bleed*) or (gingiva* N3 pocket*) or (gingiva* N3 attachment ${ }^{\star}$ )

S6 MH "Periodontitis+"

S7 periodont*

S8 (haemorrhag* N5 gum*) or (hemorrhag* N5 gum*) or (hemorrag* N5 gum) or (haemorrhag* N5 gingiva*) or (hemorrhag* N5 gingiva*) or (hemorrag* N5 gingiva*)

S9 (bleed $^{\star}$ N5 gum ${ }^{\star}$ ) or (bleed ${ }^{\star}$ N5 gingiva*)

S10 (inflamm* N5 gum*) or (inflamm* N5 gingiva*)

S11 "gum disease" or (disease* N5 gum ${ }^{\star}$ ) or (disease* N5 gingiva*)

$\mathrm{S} 12 \mathrm{~S} 1$ or S2 or S3 or S4 or S5 or S6 or S7 or S8 or S9 or S10 or S11

$\mathrm{S} 13 \mathrm{MH}$ "Mouthwashes+"

S14 mouthrins* or "mouth rins*" or mouth-rins* or rinse* or rinsing* or mouthwash* or "mouth wash ${ }^{\star}$ or mouth-wash*

S15 (oral N3 wash ${ }^{\star}$ ) or (dental N3 wash ${ }^{\star}$ ) or (mouth* N3 wash*) or (oral N3 rins ${ }^{\star}$ ) or (dental N3 rins*) or(mouth* N3 rins*)

$\mathrm{S} 16 \mathrm{~S} 13$ or $\mathrm{S} 14$ or $\mathrm{S} 15$

S17 MH "Chlorhexidine+"

S18 chlorhexidine

S19 MK-412a or "MK 412a" or MK412a

$\mathrm{S} 20 \mathrm{TI} \mathrm{CHX}$ or AB CHX

S21 (Corsodyl or Peridex or Hibident or Prexidine or Parodex or Chlorexil or Peridont or Eludril or Perioxidin or Chlorohex or Savacol or Periogard or Chlorhexamed or Nolvasan or Sebidin or Tubulicid or hibitane)

$\mathrm{S} 22 \mathrm{~S} 17$ or $\mathrm{S} 18$ or $\mathrm{S} 19$ or $\mathrm{S} 20$ or $\mathrm{S} 21$

S23 S12 and S16 and S22

The above subject search was linked to Cochrane Oral Health's filter for identifying RCTs in CINAHL:

S1 MH Random Assignment or MH Single-blind Studies or MH Double-blind Studies or MH Triple-blind Studies or MH Crossover design or MH Factorial Design 
S2 TI ("multicentre study" or "multicenter study" or "multi-centre study" or "multi-center study") or AB ("multicentre study" or "multicenter study" or "multi-centre study" or "multi-center study") or SU ("multicentre study" or "multicenter study" or "multi-centre study" or "multicenter study")

S3 TI random* or AB random*

S4 AB "latin square" or TI "latin square"

S5 TI (crossover or cross-over) or AB (crossover or cross-over) or SU (crossover or cross-over)

S6 MH Placebos

$\mathrm{S} 7 \mathrm{AB}\left(\right.$ singl $^{\star}$ or doubl ${ }^{\star}$ or trebl $l^{\star}$ or tripl $\left.{ }^{\star}\right)$ or TI (singl ${ }^{\star}$ or doubl ${ }^{\star}$ or trebl ${ }^{\star}$ or tripl $\left.l^{\star}\right)$

S8 TI blind* or AB mask* or AB blind* or TI mask ${ }^{\star}$

S9 S7 and S8

S10 TI Placebo* or AB Placebo* or SU Placebo*

S11 MH Clinical Trials

S12 TI (Clinical AND Trial) or AB (Clinical AND Trial) or SU (Clinical AND Trial)

$\mathrm{S} 13 \mathrm{~S} 1$ or $\mathrm{S} 2$ or $\mathrm{S} 3$ or $\mathrm{S} 4$ or $\mathrm{S} 5$ or $\mathrm{S} 6$ or $\mathrm{S} 9$ or $\mathrm{S} 10$ or $\mathrm{S} 11$ or $\mathrm{S} 12$

\section{Appendix 6. US National Institutes of Health Ongoing Trials Register (ClinicalTrials.gov) search strategy}

chlorhexidine AND mouthrinse AND gingivitis

chlorhexidine AND mouthwash AND gingivitis

\section{Appendix 7. World Health Organization International Clinical Trials Registry Platform search strategy}

chlorhexidine AND mouthrinse AND gingivitis

chlorhexidine AND mouthwash AND gingivitis

WHAT'S NEW

\begin{tabular}{lll}
\hline Date & Event & Description \\
\hline 13 December 2021 & Review declared as stable & $\begin{array}{l}\text { There is unlikely to be new evidence to include in this review that } \\
\text { would change the results so it will not be further updated. }\end{array}$ \\
\hline
\end{tabular}

\section{HISTORY}

Protocol first published: Issue 9, 2010

Review first published: Issue 3, 2017

\begin{tabular}{lll}
\hline Date & Event & Description \\
\hline 16 August 2010 & Amended & Amended declaration of interest statement. \\
\hline
\end{tabular}

\section{CONTRIBUTIONSOF AUTHORS}

Helen Whelton (HW) conceived the idea for the review. Patrice James (PJ), Carmel Parnell (CP), Philip Riley (PR), Thomas Lamont (TL) and Mairead Harding ( $\mathrm{MH}$ ) identified the studies to be included in the review. PJ, CP, PR, TL, MH and Andrea Cheung (AC) carried out data extraction. PJ, AC, PR and TL compiled the characteristics of included studies tables. PJ, CP, PR, MH and TL assessed the risk of bias for the included studies. Helen Worthington (HVW) statistically analysed the data. PJ, TL and AC drafted the background section. PJ, HVW and PR drafted the body of the review. $\mathrm{HW}, \mathrm{MH}, \mathrm{CP}$ and $\mathrm{AC}$ commented on and edited the draft review. HVW and PR provided advice and guidance throughout the review process.

\section{DECLARATIONS OF INTEREST}

As former Director of the Oral Health Services Research Centre, Cork, Helen Whelton has conducted clinical trials of products for GlaxoSmithKline (GSK) and she received consultancy fees in relation to this research. Mairead Harding (Deputy Director of the Oral Health Services Research Centre) was involved in the development of educational materials with regard to tooth wear/dental erosion and has 
conducted epidemiological studies and presented the findings on tooth wear and dental erosion at conferences. Fees received from GlaxoSmithKline for this work were paid to the Oral Health Services Research Centre, Cork and not to Mairead Harding. Philip Riley is a salaried member of the Cochrane Oral Health editorial team. Editorial control of the review and final decisions about the content of the review were maintained by Helen Worthington who is Co-ordinating Editor of Cochrane Oral Health. The remaining co-authors declare no conflict of interest.

\section{SOURCES OF SUPPORT}

\section{Internal sources}

- Division of Dentistry, School of Medical Sciences, Faculty of Biology, Medicine and Health, The University of Manchester, Manchester Academic Health Science Centre, UK

\section{External sources}

- Health Research Board Cochrane Fellowship Scheme, Ireland

- National Institute for Health Research (NIHR), UK

This project was supported by the NIHR, via Cochrane Infrastructure funding to Cochrane Oral Health. The views and opinions expressed herein are those of the review authors and do not necessarily reflect those of the Systematic Reviews Programme, the NIHR, the NHS or the Department of Health.

- Cochrane Oral Health Global Alliance, Other

The production of Cochrane Oral Health reviews has been supported financially by our Global Alliance since 2011 (oralhealth.cochrane.org/partnerships-alliances). Contributors over the past year have been the American Association of Public Health Dentistry, USA; the British Association for the Study of Community Dentistry, UK; the British Society of Paediatric Dentistry, UK; the Canadian Dental Hygienists Association, Canada; the Centre for Dental Education and Research at All India Institute of Medical Sciences, India; the National Center for Dental Hygiene Research \& Practice, USA; New York University College of Dentistry, USA; and NHS Education for Scotland, UK.

\section{DIFFERENCES BETWEEN PROTOCOL AND REVIEW}

In Spring 2016, in conjunction with the editorial board of Cochrane Oral Health, the decision was made to address the comparison of chlorhexidine mouthrinse with placebo, control or mechanical oral hygiene alone in this review and to report the comparison of chlorhexidine mouthrinse with other active mouthrinses in a subsequent review. This decision will enable us to comprehensively report all of the results for the objectives set out in the published protocol (James 2010) across two reviews.

A number of additional changes were made in relation to the criteria for including studies in the review after the protocol was published but early in the review process.

- Types of studies

- It was decided to exclude cross-over trials due to concerns that chlorhexidine could exert an effect beyond the washout period.

- When we wrote the protocol, we did not anticipate encountering split-mouth studies meeting the inclusion criteria because it is not possible to conduct a split-mouth study when using mouthrinse as the intervention. However, we encountered split-mouth studies comparing different scaling and root planing regimens and different periodontal surgical techniques that incorporated a chlorhexidine and placebo/control comparison. Such study designs were considered inappropriate to answer the question posed by this review and were excluded.

- Types of participants

- Individuals no longer need to have undergone periodontal treatment and be in the maintenance phase to be included. This change was made to allow us to include studies where chlorhexidine was used as an adjunct to surgical and non-surgical periodontal therapy. Individuals of any age, gender or race with periodontal disease (gingivitis or periodontitis) provided they are capable of performing mechanical oral hygiene procedures are included.

- Risk of bias

- Assessment of blinding for participants, personnel and outcome assessors was changed to allow for low and unclear risk of bias in these domains in certain situations.

The following were not explicitly addressed in the protocol and required clarification. 
- Types of intervention

- We clarified that the mechanical oral hygiene procedures must be the same in both the chlorhexidine mouthrinse and the comparison arms so that the chlorhexidine mouthrinse is the only difference between the arms to ensure that the groups are truly comparable.

- Studies where the chlorhexidine mouthrinse also contained fluoride were included.

- Studies where gum care or antigingivitis dentifrices (that do not contain chlorhexidine) are used for mechanical oral hygiene in both test and comparator arms were included.

- Studies where chlorhexidine mouthrinse formed part of a combined intervention with other agents (such as other chlorhexidine vehicles, dentifrice containing chlorhexidine, or other antigingivitis agents (e.g. cetylpyridinium chloride (CPC)) that the comparator $\mathrm{arm} / \mathrm{s}$ did not receive were excluded because the effect of the chlorhexidine could not be separated from the effect of the other active agents.

- Types of outcome

- A hierarchy to guide data extraction of gingivitis and plaque data was developed to facilitate data extraction and analysis. The main analysis however, was based on the main prespecified gingivitis index: the Gingival Index of Löe and Silness (Löe 1967; Löe and Silness 1963).

\section{NOTES}

There is unlikely to be new evidence to include in this review that would change the results so it will not be further updated.

\section{INDEX TERMS}

\section{Medical Subject Headings (MeSH)}

Chemotherapy, Adjuvant; Chlorhexidine [adverse effects] [ ${ }^{\star}$ therapeutic use]; Dental Plaque [complications] [ ${ }^{\star}$ drug therapy]; Dental Plaque Index; Dental Prophylaxis; Gingivitis [ ${ }^{*}$ drug therapy] [etiology]; Mouthwashes [adverse effects] [ ${ }^{*}$ therapeutic use]; Oral Hygiene; Publication Bias; Randomized Controlled Trials as Topic; Time Factors; Tooth Discoloration [chemically induced]

\section{MeSH check words}

Adolescent; Adult; Aged; Aged, 80 and over; Child; Female; Humans; Male; Middle Aged 